\title{
FLUID DIVERSION AND SWEEP IMPROVEMENT WITH CHEMICAL GELS IN OIL RECOVERY PROCESSES
}

\author{
Annual Report for the Period \\ May 1, 1989 -2 April 30, 1990 \\ $\mathrm{DOE} / \mathrm{BC} / 14447--8$ \\ DE91 002233 \\ By \\ Frank S. Seright \\ F. David Martin
}

April 1991

Work Performed Under Contract No. DE-FG22-89BC14447

Prepared for

U.S. Department of Energy

Assistant Secretary for Fossil Energy

Jerry Casteel, Project Manager

Bartlesville Project Manager

P. O. Box 1398

Bartlesville, OK 74005

Prepared by

New Mexico Institute of Mining and Technology

New Mexico Petroleum Recovery Research Center

Socorro, New Mexico 


\section{ABSTRACT}

This report describes progress made during the first year of the three-year project, "Fluid Diversion and Sweep Improvement with Chemical Gels in Oil Recovery Processes." The objectives of this project are to identify the mechanisms by which gel treatments divert fluids in reservoirs and to establish where and how gel treatments are best applied. Several different types of gelants are being examined, including a monomer-based gelant, several polymer-based gelants, and a colloidal silica gelant.

A resorcinol-formaldehyde gel was studied extensively. Insights obtained by studying this relatively simple gel system may be valuable when assessing the performance of more complicated gels in fluid diversion. During core experiments, residual resistance factors $\left(\mathrm{F}_{\mathrm{rr}}\right)$ were very high $\left(10^{3}-10^{4}\right)$ for gelants buffered and formed at $\mathrm{pH}=9$. As $\mathrm{pH}$ was decreased during core experiments, the gelation reaction was inhibited. In particular, as gelation $\mathrm{pH}$ decreased from 7 to $6, F_{\pi}$ values decreased sharply from high to low values (e.g., from $\$ 1000$ to 1). Tracer studies show that the fraction of the pore volume occupied by the gel generally decreases over this $\mathrm{pH}$ range. In general, $F_{\pi}$ values increased with decreased permeability. However, $F_{\pi}$ values can be significantly higher in sandstones than in less-permeable carbonate cores. A simple mathematical model was used to assess whether $\mathrm{pH}$ effects can be exploited to optimize gel placement in injection wells. Some preliminary studies were also performed to examine the effects of a resorcinol-formaldehyde gel on the flow of oil and $\mathrm{CO}_{2}$ in Berea sandstone.

Experiments were performed to probe the rheology of chromium(III)-xanthan geis and gelants in porous media. For a large fraction of the time prior to gelation, the presence of $90-\mathrm{ppm} \mathrm{Cr}{ }^{3+}$ was found not to significantly affect the rheology in porous media of a 3000-ppin xanthan solution. Residual resistance factors provided by these gels were quite low. Much larger $F_{m}$ values were obtaine ${ }^{2}$ using gels with $4000-\mathrm{ppm}$ xanthan and $154-\mathrm{ppm} \mathrm{Cr}^{3+}$ : For these gels, $\mathrm{F}_{\pi}$ values decreased significantly with increased fluid flux and could be described by a power-law relation over the flux range from 0.025 to $16 \mathrm{ft} / \mathrm{d}$. The importance of this apparent "shear-thinning" nature of residual resistance factors is discussed.

Existing theories were applied to explore the influence of diffusion, dispersion, and viscous fingering during placement of gels to modify injection profiles. This work reveals that diffusion and dispersion usually will not eliminate the need for zone isolation during gel placement in unfractured injection wells. During gel placement in parallel laboratory corefloods, diffusion and disforsion can mislead one to conclude that zone isolation is not needed in field applications. The role of a water postflush during gel placement was also investigated.

A mathematical study was performed to characterize gel placement in production wells. This work demonstrates that gelling agents can penetrate to a significant degree into all open zones--not just those with high water saturations. The damage caused by gel in oil-productive zones will depend greatly on (1) the flow geometry (linear or radial), (2) hysteresis of water-oil relative permeability curves, (3) the extent to which gels disproportionately reduce relative permeability to water more than that to oil, and (4) fluid saturations. The impact of each of these factors is discussed. 


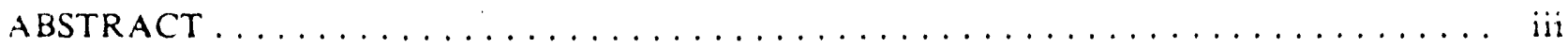

LIST OF FIGURES $\ldots \ldots \ldots \ldots \ldots \ldots \ldots \ldots \ldots \ldots \ldots \ldots \ldots \ldots \ldots \ldots \ldots$

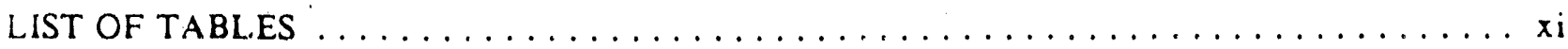

ACKNOWLEDGEMENTS $\ldots \ldots \ldots \ldots \ldots \ldots \ldots \ldots \ldots \ldots \ldots \ldots \ldots \ldots$

EXECUTIVE SUMMARY $\ldots \ldots \ldots \ldots \ldots \ldots \ldots \ldots \ldots \ldots \ldots \ldots \ldots \ldots \ldots \ldots \ldots$

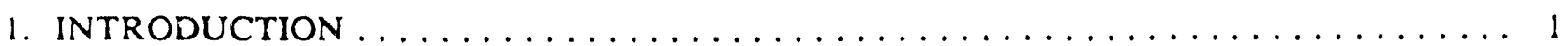

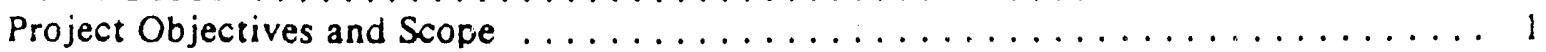

Project Task Areas $\ldots \ldots \ldots \ldots \ldots \ldots \ldots \ldots \ldots \ldots \ldots \ldots \ldots \ldots \ldots \ldots$

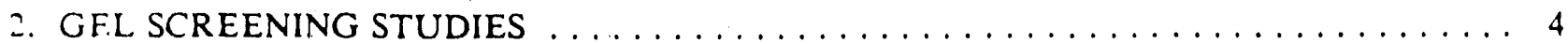

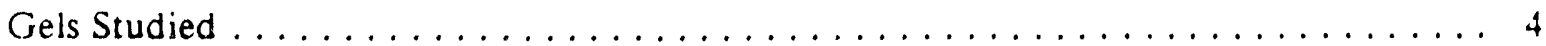

Experimental Procedures $\ldots \ldots \ldots \ldots \ldots \ldots \ldots \ldots \ldots \ldots \ldots \ldots \ldots \ldots$

Gelation at Neutral $\mathrm{pH} \ldots \ldots \ldots \ldots \ldots \ldots \ldots \ldots \ldots \ldots \ldots \ldots$

$\mathrm{pH}$ of Brine in Contact With Compressed $\mathrm{CO}_{2} \ldots \ldots \ldots \ldots \ldots \ldots$

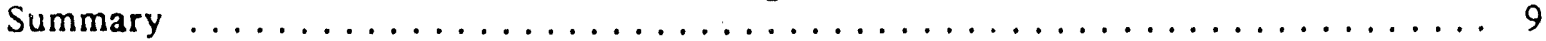

3. IMPACT OF GELATION pH, ROCK PERMEABILITY, AND LITHOLOGY ON THE PERFORMANCE OF A MONOMER-BASED GEL $\ldots \ldots \ldots \ldots \ldots \ldots \ldots \ldots \ldots 10$

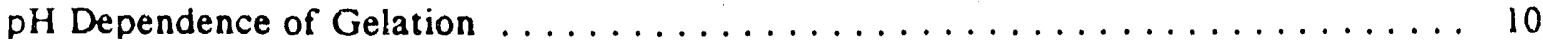

Chemical Transport in Porous Media Prior to Gelation $\ldots \ldots \ldots \ldots \ldots \ldots \ldots \ldots$

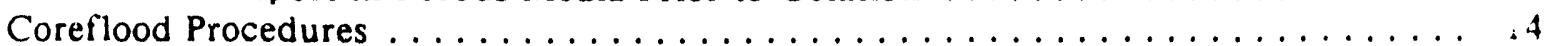

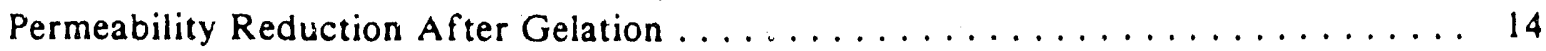

Exploiting pH to Optimize Gel Placement ....................... 19

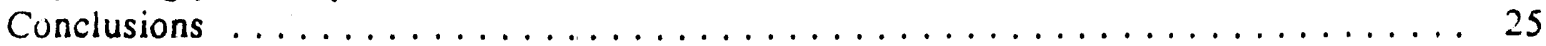

4. A PRELIMINARY STUDY OF PERMEABILITY REDUCTION FOR $\mathrm{CO}_{2}$ AND WATER USING A RESORCINOL-FORMALDEHYDE GEL $\ldots \ldots \ldots \ldots \ldots \ldots \ldots$

Flow of Brine and $\mathrm{CO}_{2}$ Through Berea Prior to Gel Placement $\ldots \ldots \ldots \ldots \ldots \ldots$

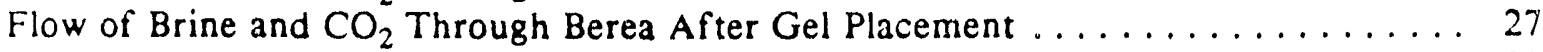

Summary ...................................... 29

5. A PRELIMINARY STUDY OF PERMEABILITY REDUCTION FOR OIL AND WATER

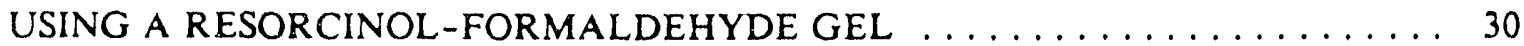

Effect of Flow-Direction Reversal on End-Point Permeabilities Prior to Gel Placement ............................. 30

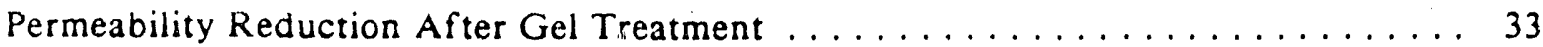

Summary ............................... 33

6. RHEOLOGY OF CYRONIUM(III)-XANTHAN GELS AND GELANTS IN POROUS

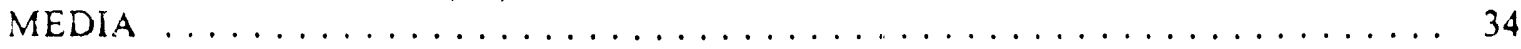

Gelant Rheclogy in Porous Media Prior to Gelation $\ldots \ldots \ldots \ldots \ldots \ldots \ldots \ldots$

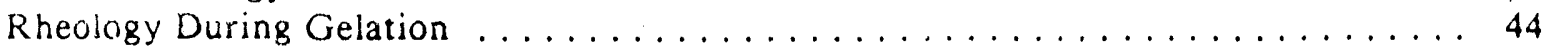

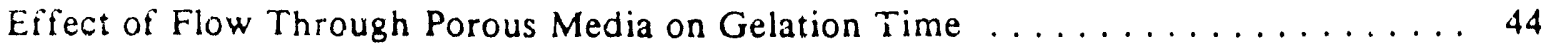


Rheology During Brine Injection After Gelation $\ldots \ldots \ldots \ldots \ldots \ldots \ldots \ldots$

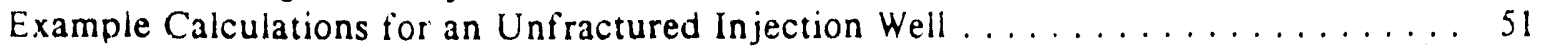

Conclusions ..... ........................... 54

7. IMPACT OF DIFFUSION, DISPERSION, AND VISCOUS FINGERING ON GEL

PLACEMENT IN INJECTION WELLS $\ldots \ldots \ldots \ldots \ldots \ldots \ldots \ldots \ldots \ldots \ldots$

Gelant Penetration in Oil-Productive Strata $\ldots \ldots \ldots \ldots \ldots \ldots \ldots \ldots \ldots \ldots$

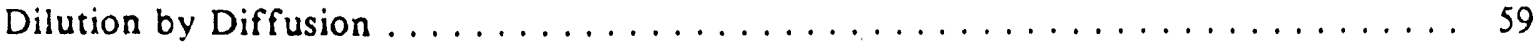

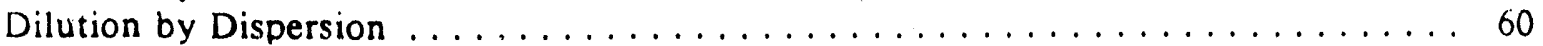

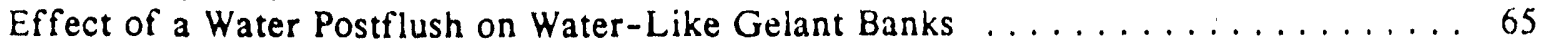

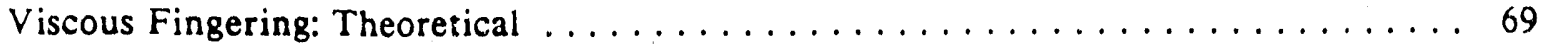

Viscous Fingering: Experimental Verification $\ldots \ldots \ldots \ldots \ldots \ldots \ldots \ldots \ldots$

Conclusions ................................ 79

8. AN EXAMINATION OF FLOW-PROFILE CHANGES FOR FIELD APPLICATIONS OF

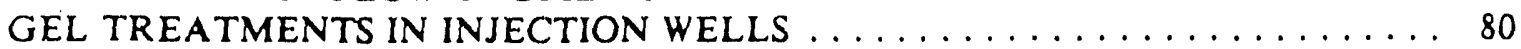

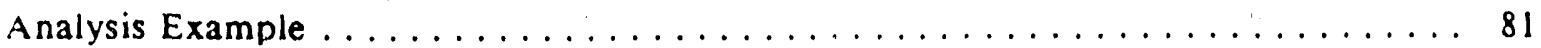

Analysis Summary for 43 Pairs of Injection Profiles $\ldots \ldots \ldots \ldots \ldots \ldots \ldots$

Field Work Needed to Resolve the Issue $\ldots \ldots \ldots \ldots \ldots \ldots \ldots \ldots \ldots$

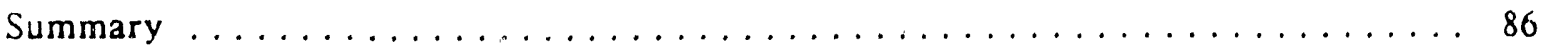

9. PLACEMENT OF GELS IN PRODUCTION WELLS $\ldots \ldots \ldots \ldots \ldots \ldots \ldots \ldots \ldots$

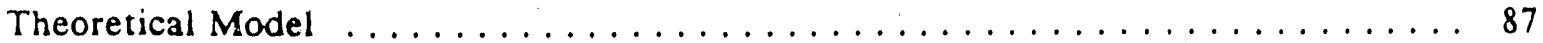

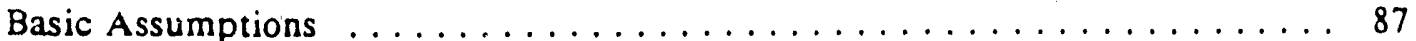

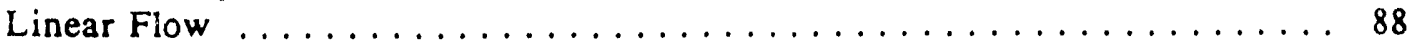

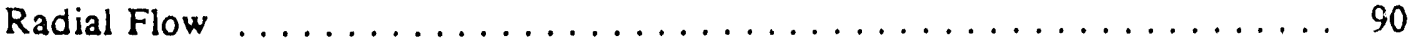

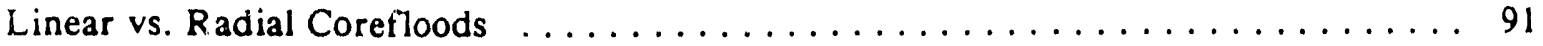

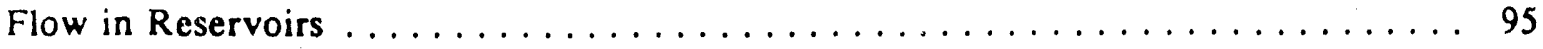

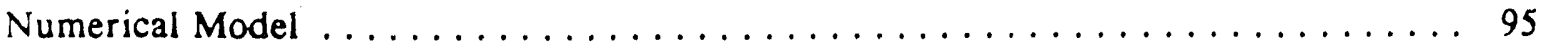

Relative Permeability Changes After Treatment $\ldots \ldots \ldots \ldots \ldots \ldots \ldots \ldots \ldots$. $\ldots \ldots$ ?

Productivity Loss After Treatment . . . . . . . . . . . . . . . . . . . . 101

Hysteresis of Oil and Water Relative Permeability Curves .......... 101

Productivity Loss After Gelation ... . . . . . . . . . . . . . . 103

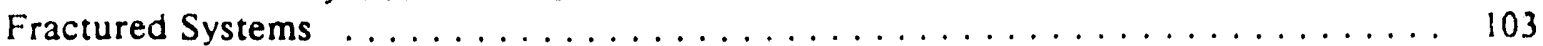

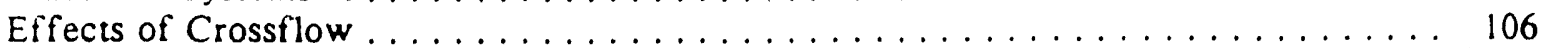

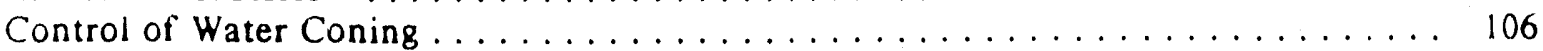

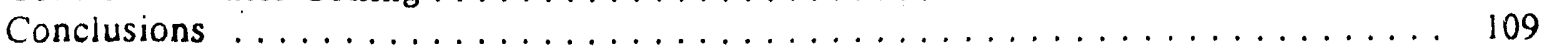

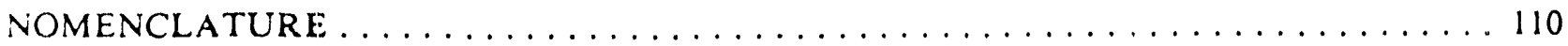

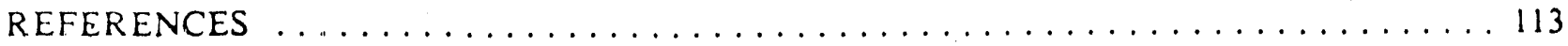

APPENDICES

A - PROCEDURE FOR FABRICATION OF TWO-DIMENSIONAL

GLASS MICROMODELS $\ldots \ldots \ldots \ldots \ldots \ldots \ldots \ldots \ldots \ldots \ldots \ldots \ldots \ldots \ldots \ldots$

B - GELATION STUDIES AT THE OPTIMUM REACTION pH $\ldots \ldots \ldots \ldots \ldots \ldots$

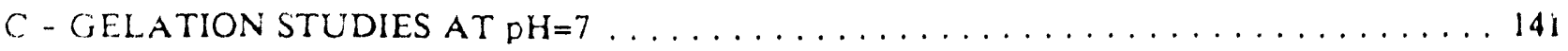

D - RESULTS FROM CORE EXPERIMENTS WITH RESORCINOL-

FORMALDEHYDE GELANTS . 


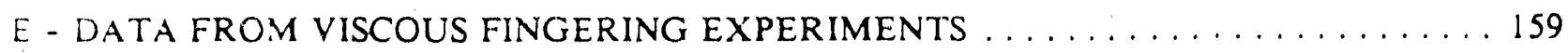

F-FORTRAN CODING FOR THEORETICAL MODEL: RADIAL FLOW $\ldots \ldots \ldots \ldots$

G -FORTRAN CODING FOR THEORETICAL MODEL: LINEAR FLOW $\ldots \ldots \ldots \ldots$

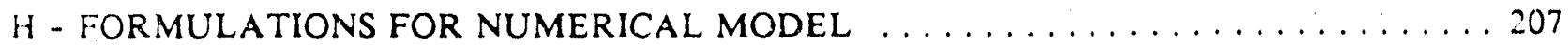




\section{LIST OF FIGURES}

Figure $1 \quad$ Compositions of Resorcinol and Formaldehyde $\ldots \ldots \ldots \ldots \ldots \ldots \ldots \ldots$

Figure 2 Reaction of Resorcinol with Formaldehyde $\ldots \ldots \ldots \ldots \ldots \ldots \ldots \ldots$

Figure $3 \quad$ Buffer Capacities of Resorcinol and Formaldehyde $\ldots \ldots \ldots \ldots \ldots \ldots$

Figure $4 \quad F_{\pi}$ Value at $0.025 \mathrm{ft} / \mathrm{d}$ After Injecting Brine at the Maximum Flux Specified on the Abscissa ........................... 17

Figure 5 Tracer Results during Brine Injection Before vs. After Gel Placement in 57-md

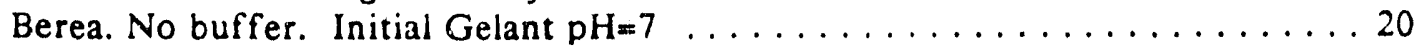

Figure 6 Tracer Results during Brine Injection Before vs. After Gel Placement in 63-md Berea. Phosphate buffer. Gelant $\mathrm{pH}=7 \ldots \ldots \ldots \ldots \ldots$

Figure 7 Tracer Results during Brine Injection Before vs. After Gel Placement in 7 -md Limestone. Phosphate buffer. Gelant $\mathrm{pH}=7 \ldots \ldots \ldots \ldots$

Figure $8 \quad$ Best-Case Profile Improvement in Radial Flow with a Weak Gel $\left(F_{r}=10\right) \ldots 24$

Figure $9 \quad$ Best-Case Profile Improvement in Radial Flow with a Strong Gel $\left(F_{r}=1000\right) \ldots 24$

Figure $10 \quad$ Results from Tracer Studies during $\mathrm{CO}_{2}$ Experiments $\ldots \ldots \ldots \ldots \ldots$

Figure 11 Tracer Breakthrough Curves from Tracer Studies during Oil/Water

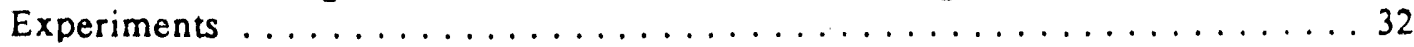

Figure 12 Tracer Breakthrough Curves with/without Residual Oil Present ........ 32

Figure 13 Effect of $\mathrm{Cr}^{3+}$ and Flow through 293-md Berea on the Viscosity of

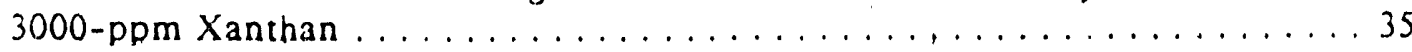

Figure 14 Effect of $\mathrm{Cr}^{3+}$ on Rheology of 3000-ppm Xanthan in 293-md Berea ...... 35

Figure 15 Effect of $\mathrm{Cr}^{3+}$ and Flow through 164-md Berea on the Viscosity of

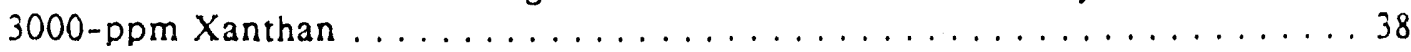

Figure 16 Effect of $\mathrm{Cr}^{3+}$ on Rheology of 3000-ppm Xanthan in 164-md Berea ...... 39

Figure 17 Effect of $\mathrm{Cr}^{3+}$ on Rheology of $3000-\mathrm{npm}$ Xanthan in 531-md Berea with

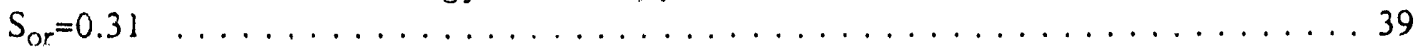

Figure 18 Residual Resistance Factor vs. Flux in 77-md Berea. Initial Gelant pH=4 . . 47

Figure 19 Residual Resistance Factor v:. Flux in 66-md Bereu. Initiai Gelant pH=7 . . . 47

Figure 20 Residual Resistance Factor vs. Flux in 483-m f Berea. Initial Gelant $p H=7 \ldots 47$ 
Figure 21 Tracer Effluent Curves During Brine Injection Before vs. After Gel Placement

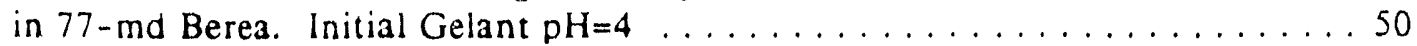

Figure 22 Tracer Effluent Curves During Brine Injection Before vs. After Gel Placement

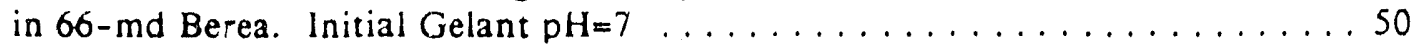

Figure 23 Tracer Effluent Curves During Brine Injection Before vs. After Gel Placement

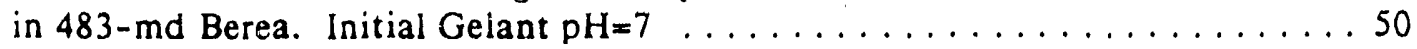

Figure 24 Relative Penetration of Fluids in an Unfractured Injection Well with Multiple, Noncommunicating Layers ................... 57

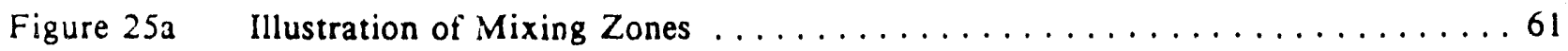

Figure 25b Concentration Profile at the Interface Between the Water Bank and the Gelling-

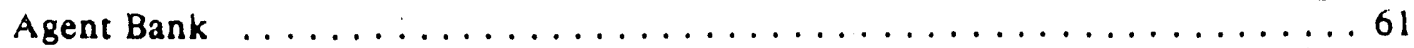

Figure $26 \quad$ Length of Mixing Zone Due to Diffusion $\ldots \ldots \ldots \ldots \ldots \ldots \ldots \ldots \ldots$

Figure $27 \quad$ Effect of Dispersion on Bank Size $\ldots \ldots \ldots \ldots \ldots \ldots \ldots \ldots \ldots \ldots \ldots \ldots \ldots$

Figure 28 Impact of Diffusion and Dispersion on Gel in Unfractured Injection Wells . . . 66

Figure 29 Exploiting Diffusion and Dispersion to Prevent Gelation in Layer $2 \ldots \ldots$. . . 67

Figure $30 \quad$ Impact of Diffusion and Dispersion during Parallel Linear Corefloods . . . . 67

Figure $31 \quad$ Illustration of a Water Postflush $\ldots \ldots \ldots \ldots \ldots$

Figure 32 Thinning of the Gelant Bank during a Water Postflush in Radial Flow . . . 68

Figure $33 \quad$ Effect of a Water Postflush on Injectivity $\ldots \ldots \ldots \ldots \ldots \ldots$

Figure $34 \quad$ Illustration of Viscous Fingering $\ldots \ldots \ldots \ldots \ldots \ldots \ldots \ldots \ldots \ldots \ldots$

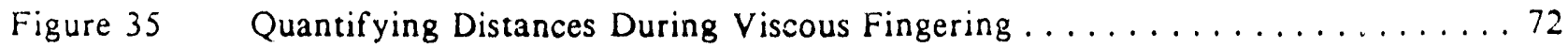

Figure 36 Sensitivity of Finger Breakthrough to Assumed Resistance Factor in the Fingered Zone: Radial Flow .................... 74

Figure 37 Sensitivity of Finger Breakthrough to Resistance Factor of the Viscous Fluid:

Radial Flow ............................ 74

Figure 38 Sensitivity of Finger Breakthrough to Assumed Resistance Factor in the

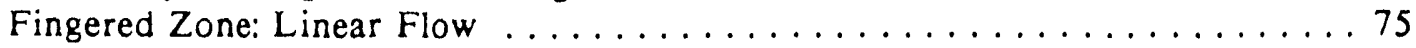

Figure 39 Sensitivity of Finger Breakthrough to Resistance Factor of the Viscous Fluid:

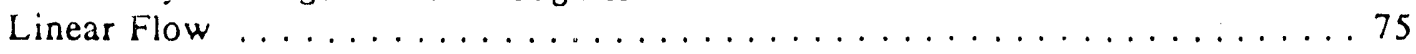

Figure $40 \quad$ Important Measurements during Viscous Fingering Experiments . . . . . . 77 
Figure $41 \quad$ Injection Profiles from Chang $e t$ al $\ldots \ldots \ldots \ldots \ldots \ldots \ldots \ldots \ldots$

Figure $42 \quad$ Injection Profiles with Flow Percentages $\ldots \ldots \ldots \ldots \ldots \ldots \ldots$

Figure $43 \quad$ Saturation Profile in Layer $i$ during the Placement Process $\ldots \ldots \ldots \ldots 9$

Figure $44 \quad$ Radial vs. Linear Parallel Corefloods; Degree of Penetration into Core i . . . . 93

Figure 45 Effect of Oil/Water Viscosity Ratio on Degree of Penetration into Core i . . . 94

Figure 46 Degree of Penetration into Core i; Effects of End-Point Permeability to

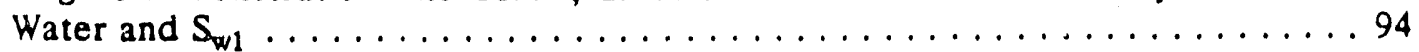

Figure 47 Effect of $\Psi_{i}$ Variations on Degree of Penetration into Layer $i \ldots \ldots 96$

Figure 48 Theoretical Model vs. Numerical Model; Degree of Penetration into Core i: Radial Flow .............................. 98

Figure 49 Oil and Water Relative Permeability Curves Before and After Gel Treatment . . 99

Figure $50 \quad$ Water Fractional Flow Curves Before and After Gel Treatment . . . . . . . 99

Figure $51 \quad$ Cil and Water Relative Permeability Curves with/without Hysteresis . . . . . 102

Figure 52 Effect of Hysteresis of Relative Permeability Curves on Productivity Loss . . 102

Figure 53a Effects of Depth of Penetration and $\mathrm{S}_{\mathrm{wi}}$ on Productivity Retained af ter Gel

Treatment ............................... 104

Figure 53b Productivity Retained after Gel Treatment with Hysteresis of Relative

Permeability Curves .......................... 104

Figure 54 Effect of $\Psi_{i}$ Variations on Degree of Penetration in Vertically

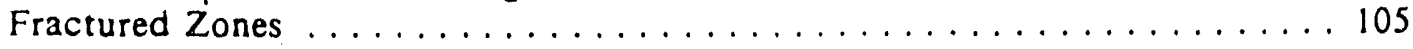

Figure 55a Effects of Degree of Penetration and $\mathrm{S}_{\mathrm{wi}}$ on Productivity Loss after Gel Treatment in Vertically Fractured Zones ................... 107

Figure 55b Productivity Retained after Gel Treatment with Hysteresis of Relative Permeability Curves in Vertically Fractured Zones . . . . . . . . . . . . 107 


\section{LIST OF TABLES}

Table 1

Table 2

Table 3

Table 4

Table 5

Table 6

Table 7

Table 8

Table 9

Table 10

Table 11

Table 12

Table 13

Table 14

Table 15

Table 16

Table 17

Table 18

Table 19

Table 20

Table 21
Summary of Gelation Results at Optimum $\mathrm{pH} \ldots \ldots \ldots \ldots \ldots \ldots$

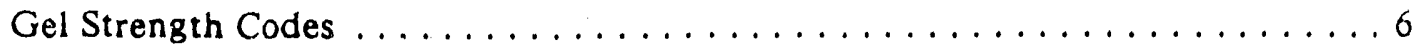

Summary of Gelation Results at $\mathrm{pH}=7 \ldots \ldots \ldots \ldots \ldots \ldots \ldots \ldots$

$\mathrm{pH}$ Values of $0.5 \% \mathrm{KCl}$ Brine in Contact with Rock and Compressed $\mathrm{CO}_{2} \ldots \ldots 9$

Results from Brine Injection After Gel Placement in 63-md Berea Sandstone . . 15

Results from Brine Injection After Gel Placement in 49 -md Berea Sandstone . . 16

Summary of Results from Brine Injection After Gelation $\ldots \ldots \ldots \ldots \ldots \ldots$

Summary of $\mathrm{CO}_{2}$ Coreflood, $105^{\circ} \mathrm{F} \ldots \ldots \ldots \ldots \ldots \ldots \ldots \ldots$

Rock and Fluid Properties for Oil/Water Experiments . . . . . . . . . . 30

Effect of Flow-Direction Reversal on End-Point Permeabilities Prior to

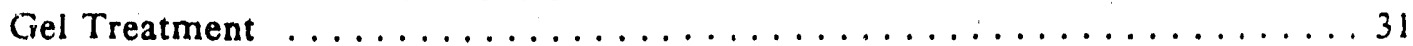

Summary of the Results of Tracer Studies During Oil/Water Experiments . . . . 31

Rheology of 3000-ppm Xanthan (without $\mathrm{Cr}^{3+}$ ) in 293-md Berea Sandstone . . 36

Rheology of 3000-ppm Xanthan with $90-\mathrm{ppm} \mathrm{Cr}^{3+}$ in 293-md Berea Sandstone 37

Rheology of $3000-p p m$ Xanthan (without $\mathrm{Cr}^{3+}$ ) in 164 -md Berea Sandstone . . 40

Rheology of 3000-ppm Xanthan with 90-ppm $\mathrm{Cr}^{3+}$ in 164-md Berea Sandstone 41

Rheology of 3000-ppm Xanthan (without $\mathrm{Cr}^{3+}$ ) in Berea Sandstone with a

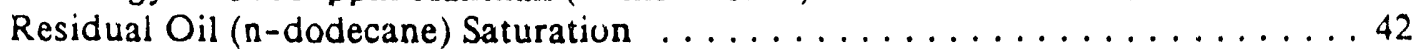

Rheology of 3000-ppm Xanthan with 90-ppm Cr${ }^{3+}$ in Berea Sandstone with

a Residual Oil (n-dodecane) Saturation . ...............43

Gelation Onset for $\mathrm{Cr}^{3+}-$ Xanthan Gelants $\ldots \ldots \ldots \ldots \ldots \ldots$

Residual Resistance Factors After Placement and Gelation in 77-md Berea

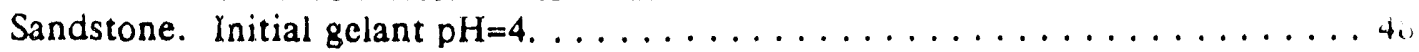

Residual Resistance Factors After Placement and Gelation in 66-md Berea

Sandstone. Init.ul getant $\mathrm{pH}=7$.

Residual Resistance Factors After Placement and Gelation in 483-md Berea Sandstone. Initial gelant $\mathrm{pH}=7$. 
Table 22 Results from Parallel Linear Floods $\ldots \ldots \ldots \ldots \ldots \ldots \ldots \ldots$

Table 23 Comparison of Dispersion Predictions for Linear and Radial Flow ... . . . 64

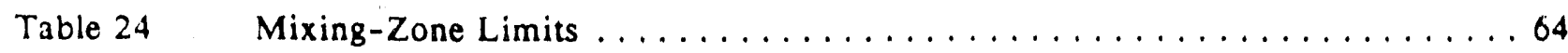

Table $25 \quad$ Surrmary of Viscous-Fingering Results $\ldots \ldots \ldots \ldots \ldots \ldots \ldots$

Table 26 Published Injection Profiles: Before versus After Gel Treatments . . . . . . . 84

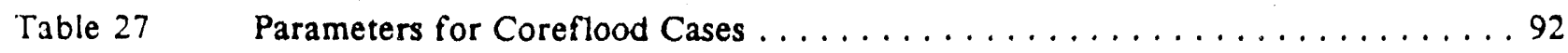

Table $28 \quad$ Parameters for Unf ractured 5 -Spot Cases $\ldots \ldots \ldots \ldots \ldots \ldots \ldots \ldots \ldots$

Table 29 Parameters Used When Comparing the Theoretical and Numerical Models . . . . 92

Table $30 \quad$ Parameters After Gelation $\ldots \ldots \ldots \ldots \ldots \ldots \ldots \ldots \ldots$

Table $31 \quad$ Parameters for Hysteresis Cases $\ldots \ldots \ldots \ldots \ldots \ldots \ldots \ldots$

Table $32 \quad$ Parameters for Productivity Loss Calculations $\ldots \ldots \ldots \ldots \ldots \ldots$ 


\section{ACKNOWLEDGEMENTS}

This work was financially supported by the U.S. Department of Energy, the New Mexico Research and Development Institute, Amoco, Conoco, Elf Aquitaine, Marathon, Oryx Energy Co., OXY USA, Phillips Petroleum, Shell Development Co., and Texaco. This support is gratefully acknowledged. The mathematical modeling portions of this work were partially supported by NCSATRA $890079 \mathrm{~N}$ and utilized the CRAY X-MP/48 system at the National Center for Supercomputing Applications at the University of Illinois at Urbana-Champaign. Richard Schrader performed most of the corefloods, was instrumental in the design and construction portions of the project, and also participated in much of the other experimental work. His contributions are greatly appreciated. We also appreciate the efforts of Michael Carr, who performed the parallel beadpack floods and who developed the improved procedures for fabricating micromodels. We acknowledge helpful discussions with Dr. Norman Morrow, Jill Buckley, Mary Graham, John Oelfke, and Sean French when developing the micromodel techniques. Dr. Jenn-Tai Liang was principally responsible for performing and reporting the experimental and theoretical work with gel treatments in production wells (Sections 5 and 9). Professor Robert $L$. Lee performed the simulation of gel treatments in production wells and also participated in many helpful discussions. We also thank Jeff Albers, John Hagstrom, Peter Lewis, Tommy Morris and Dennis Newell for their help during some of the experiments. The authors thank the staff of the New Mexico Petroleum Recovery Research Center (notably, Karen Bohlender, Toni Johnson, and Dawn Anderson) for their help in preparing and reviewing this report. We especially appreciate the thorough review of the manuscript that was provided by Julie Ruff. 


\section{EXECUTIVE SUMMARY}

The objectives of this project are to identify the mechanisms by which gel treatments divert fluids in reservoirs and to establish where and how gel treatments are best applied. Several different types of gelants are being examined. This research is directed at gel applications in water injection wells, in production wells, and in high-pressure gas floods. The work will establish how the flow properties of gels and gelling agents are influenced by permeability, lithology, and wettability. Other goals include determining the proper placement of gelants, the stability of in-place gels, and the types of gels required for the various oil recovery processes and for different scales of reservoir heterogeneity.

This report describes progress made during the first year of this three-year study.

\section{Srreening Studies}

Screening tests were verformed to establish gelation times, gel strengths, and gel stabilities for the gel systems that are being studied in the project. Several different types of gelants were examined, including a monomer-based gelant, several polymer-based gelants, and two colloidal silica gelants. Gel stabilities were determined over a 30-day period as a function of temperature (room temperature to $200^{\circ} \mathrm{F}$ ) and $\mathrm{pH}$ of water in contact with the gel (water pH values of 3, 7, and 12,5). For each gelant, one set of studies was performed on gels that were allowed to form at the optimum $\mathrm{pH}$ for the gelation reaction (i.e., the $\mathrm{pH}$ specified by the gelant supplier). A second set of studies was performed on gels that were formed at $\mathrm{pH}=7$. These latter studies were initiated because reservoir rocks may force in-situ gelation reactions to occur at neutral pH, even though the gelant was in jected at some other pH.

Some cursory experiments were performed to determine the $\mathrm{pH}$ of brine in contact with rock and compressed $\mathrm{CO}_{2}$. The results indicate that the $\mathrm{pH}$ of a $0.5 \% \mathrm{KCl}$ brine in contact with $\mathrm{CO}_{2}$ at $1500 \mathrm{psi}$ and $105^{\circ} \mathrm{F}$ will be in the range from 4.4 to 5.1 . This $\mathrm{pH}$ is fairly insensitive to the presence or absence of rock (Berea sandstone, Indiana limestone, or San Andres dolomite).

\section{Studies Using a Resorcinol-Formaldehyde Gel}

For many of the geis that are currently used in enhanced oil recovery, the gelation reaction is very sensitive to $\mathrm{pH}$. Often, an optimum pH exists at which the strongest gels form. At other $\mathrm{pH}$ values, weak geis may form, or gelation may not occur at all. Commonly, the optimum pH for gelation is not near neutral pH. Also, most gel formulations have very little buffering capacity (i.e., their $\mathrm{pH}$ may be changed very easily). On the other hand, clays and other minerals in reservoir rocks usually have a tremendous capacity to buffer aqueous solutions near neutral pH. Thus, even though a gelant is injected at the optimum $\mathrm{pH}$ for gelation, the rock may quickly change the ${ }_{F} \mathrm{H}$ to a less optimum value.

We performed an experimental investigation of the effects of gelation $\mathrm{pH}$ on the performance of a resorcinol-formaldehyde gel. This gel has been used in field applications and was chosen for study because its placement in porous media is not complicated by some of the factors that influence placement of polymeric gelling agents (i.e., permeability- and lithology-dependent retention of polymers and metallic crosslinkers). 
Beaker studies and core experiments were performed using both unbuffered gelants and gelants that were buffered at a variety of $\mathrm{pH}$ values. The product formed by the reaction of resorcinol with formalciehyde depends on $\mathrm{pH}$. During studies of gelfison in beakers, the strongest resorcinol-form: ldehyde gels are formed at $\mathrm{pH}=9$. At $\mathrm{pH}=9$, a strong gel is formed that is clear and red. The inherent permeability to water for this gel (no rock) was found to be $6 \mu \mathrm{D}$. As the initial $\mathrm{pH}$ is decreased, gel formation becomes less perfect. With an initial pH of 7 , an opaque orange- white $\mathrm{gel}$ is formed during beaker tests, and some free water remains after the reaction. As initial pH value is decreased below 7, the final ratio of free water to gel increases.

During core experiments, residual resistance factors are very high $\left(10^{3}-10^{4}\right)$ for gelants buffered and formed at $\mathrm{pH}=9$. Tracer studies reveal that these gels occupy $87 \%$ to $99 \%$ of the available pore volume. As pH is decreased during core experiments, the gelation reaction is inhibited. In particular, as gelation $\mathrm{pH}$ decreases from 7 to 6 , residual resistance factors decrease sharply from high to low values (e.8., from $\approx 1000$ to 1). Tracer studies show that the fraction of the pore volume occupied by the gel gencrally decreases over this $\mathrm{pH}$ range. In many core experiments, the results suggest that upon first exposure to a given fluid velocity, a certain amount of gel brejks down to allow a flow path through the porous medium. Flow of brine through this porous medium then appears more or less Newtonian until the previuus maximum in fluid velocity is cxceeded.

In general, residual resistance factors $\left(F_{\pi}\right)$ increased with decreased permeability. However, $F_{\pi}$ values can be significantly higher in sandstones than in less-permeable carbonate cores. A simple mathematical model was used to assess whether $\mathrm{pH}$ effects can be exploited to optimize gel placement in injection wells. Our results suggest that $\mathrm{pH}$ ef fects usually will not help much in eliminating the need for zone isolation during gel placement in radial flow (unfractured injection wells). Insights obtained by studying this relatively simple gel system may be valuable when assessing the performance ur more complicated gels in fluid diversion.

Some preliminary studies have also been performed to examine the effects of a resorcinolformaldehyde gel on the flow of oil and $\mathrm{CO}_{2}$ in Berea sandstone. The main value of these studies is in establishing procedures that will be used in future experiments.

\section{Rhealogy of Chromium(III)-Xanthan Gels and Gelants in Porous Media}

Experiments were performed to probe the rheology of chromium(III)-xanthan gels and gelants in porous media. For a large fraction of the time prior to gelation, the presence of $90-\mathrm{ppm} \mathrm{Cr}^{3+}$ was found not to significantly affect the rheology in porous media of a $3000-p p m$ xanthan solution.

For gel formulations containing 3000-ppm xanthan and 90-ppm $\mathrm{Cr}^{3+}$, residual resistance factors in Berea sandstone were not much greater than those for xanthan solutions without $\mathrm{Cr}^{3+}$. We suspect that clay and carbonate minerals in Berea forced the gelation reaction to occur near neutral $\mathrm{pH}$ rather than at the injection $\mathrm{pH}(3.8)$. In beaker tests, rigid gels are formed from $\mathrm{Cr}^{3+}-\mathrm{xanthan}$ gelants at $\mathrm{pH} \approx 4$, but gelation is nut apparent for the same formulation at $\mathrm{pH}=7$. Previous researchers reported large residual resistance factors for more dilute $\mathrm{Cr}^{2}+x$ ninan gels in clean sandpacks. The discrepancy between our results and those of previous researchers may be explained in that the lack of clay and carbonate minerals in sandpacks may have allowed gelation to occur at low pH values rather than at neutral $\mathrm{pH}$. Thus, the buffering action of reservoir rocks should be considered when evaluating gel performance in we icboratory.

Additional siudies were performed with a gelant that contained 4000-ppm xanthan and $154-$

$\mathrm{ppm} \mathrm{Cr}^{3+}$. With this composition, a rigid gel was formed in a beaker at $\mathrm{pH}=4$, but gelation was not evident at $\mathrm{pH}=7$. Even so, the composition injected at $\mathrm{pH}-7$ provided substantial residual resistance 
lactors $(30-714)$ in Berea sandstone. Tracer studies indicated that the gel occupied between $0 \%$ and $45 \%$ of the original pore volume, depending on the initial $\mathrm{pH}$ of the gelant and the core permeability. Tracer studies also revealed that the gel increased dispersivity values in Berea by factors ranging from 5.5 to 17.8. For this gel in 483 -md Berea, residual resistance factors were quite high $(50-714)$ even though tracer studies indicated that the pore volume occupied by the gel was near zero. Perhaps small gel particles lodge in pore throats-thereby dramatically reducing brine permeability without occupying much volume. Residual resistance factors provided by these gets decreased significantly with increased fluid flux and coule se described by a power-law relation over the flux range from 0.025 to $16 \mathrm{ft} / \mathrm{d}$.

Laboratory measurements of gel properties in 66-md Berea and in 483-md Berea were used during example calculations to show that the apparent "shear-thinning" nature of residual resistance factors will not eliminate the need for zone isolation during gel placement in unfractured injection wells.

Impact of Diffusion, Dispersion, and Viscous Fingering on Gel Placement in Injection Wells

A key issue in gel technology is how to place gels in "thief" zones without damaging oilproductive zones. Simple calculations using the Darcy equation indicate that gel treatments in unfractuied in jection wells are not likely to improve in jection protiles unless zones are isolated during gel placement. We explored the influence of diffusion, dispersion, and viscous fingering during placement of gels to modify injection profiles. In particular, these phenomena are examined to determine whether they can be exploited to optimize gel placement. In concept, diffusion and dispersion could dilute gelling agents enough to prevent gelation in less-permeable, oil-productive zones while still allowing a gel plug to form in watered-out, high-permeability streaks. However, this study reveals that diffusion and dispersion usually will not eliminate the need for zone isolation during gel placement in unfractured injection wells. During gel placement in parallel laboratory corefloods, diffusion and dispersion can mislead one to conclude that zone isolation is not needed in field applications.

During this study, we also investigated the role of a water postflush during gel placement. A postflush can provide additional mixing and thinning of the gelant banks by displacing gelling agents away from the wellbore prior to gelation. One goal of this work was to determine whether viscous fingering from a water postflush could be exploited during placement of a viscous gelant. Both theory and experiments indicate that viscous fingers usually will break through the viscous bank in the most-permeable layer first. However, at the time of this breakthrough, the fingers will have traversed most of the viscous bank in a less-permeable layer. This finding is discussed with respect to its relevance to field applications of gel treatments and traditional polymer floods.

The analyses provided here and eisewhere suggest that zone isolation can significantly improve the performance of gel treatments in some applications. (These include unfractured injection wells with noncommunicating layers. Of course, cement squvezes can be equally effective in these applications.) If zone isolation is not fersible, then our analyses raise doubts that gel treatments can be effective in unfractured injection wells. Literature reports of field applications to date do not provide enough information to confirm or contradict these analyses. Iigei treatments can be effective in uniractured wells without using zore isolation, then this success is due to some phenomenon that hias yet to be ioentiöieo. 


\section{Placement of Gels in Production Wells}

Applications of near-wellbore gel treatments in production wells are intended to reduce water production without sacrificing oil production. During gel placement, much of the gel formulation will enter zones that are responsible for the excess water production. However, some of this fluid may penetrate into and damage oil-productive zones.

We performed a critical examination of gel placement in production wells. An analysis was developed using fractional flow concepts to determine the degree of penetration of gelling agents into oil-productive strata as well as into water-source zones. Variables examined include permeability contrast. oil/water viscosity ratio, initial oil saturations, relative permeability characteristics, and gelling-agent properties. A near-wellbore simulator was developed to confirm predictions that were made using the analytical model.

This work demonstrates that gelling agents can penetrate to a significant degree into all open zones--not just those with high water saturations. The damage caused by gel in oil-productive zones will depend greatly on (1) the flow geometry (linear or radial), (2) hysteresis of water-oil relative permeability curves, (3) the extent to which geis disproportionately reduce relative permeability to water more than that to oil, and (4) fluid saturations. The impact of each of these factors is discussed.

Particular attention is paid to the impact of gel treatments that reduce the relative permeability to water more than that to oil. Induced changes in the relative permeability curves nearwellbore will not necessarily enhance oil recovery from a particular zone. Depending on the steadystate fractional flows of fluid outside of the gel-treated region, oil production could be impaired even though the gel reduces water permeability without affecting oil permeability. The principal ad vantage of the disproportionate reduction of the water and oil relative permeabilities is in reducing the need for zone isolation during gel placement. Realizing this advantage generally requires high fractional oil flow from the zone(s) of interest.

Examples are provided to illustrate and contrast situations where gels are/are not expected . . damage oil productivity. Cases considered include fractured and unfractured production wells and wells with and without water coning problems. The analysis provides an explanation for why some of the most successful applications of geis have been in fractured wells that are produced by bottomwater drive. With the right properties, these gels can significantly increase the critical rate for water coning. 


\section{INTRODUCTION}

In any oil recovery process, large-scale heterogeneities, such as fractures, channels, or highpermeability streaks, can cause breakthrough of injected fluid which will reduce oil recovery' efficiency. In enhanced recovery projects, this problem is particularly acute because of the cost of the in jected fluids.

Crosslinked-polymer treatments (gel treatments) were developed to reduce channeling of fluids through fractures and streaks of very high permeability. These treatments have been successfully applied to both injection wells and production wells. The success in injection wells has been demonstrated in the North Stanley polymer flood. ${ }^{\perp}$ Here, gel treatments were applied to plug fractures prior to implementing a traditional polymer flood. A large portion of the tertiary oil recovered in this project was attributed to the gel treatments rather than to the polymer flood. Gel treatrnents in production wells have worked particularly well in reducing water coning in the bottomwater-drive Arbuckle reservoirs of Kansas. ${ }^{2}$ Gel treatments have also been applied to reduce channeling in miscible floods. 3,4

Near-wellbore applications of crosslinked-polymer treatments in in jection wells experienced explosive growth between 1980 and 1986. At the peak of activity, 35\% of the enhanced oil recovery (EOR) projects in the United States were pclymer projects. ${ }^{5}$ A close examination of various literature sources (Enhanced Recovery Week. Oil \& Gas Journal, and others) reveais that about $60 \%$ of these polymer projects were gel treatments rather than traditional polymer floods. ${ }^{6}$ Although many projects have been very successful, many other gel projects have been technical failures. Even though $20 \%$ of ail EOR projects were gel treatments, they have been responsible for less than two percent of the total EOR production in the United States. 5,6 One study conducted in 1985 revealed that less than $45 \%$ of near-wellbore gel treatments were successful. ${ }^{7}$ A second study of over 100 well treatments within one company revealed a $48 \%$ success ratio. ${ }^{7}$ In part, the success of gel projects has been sporadic because the science and technology base did not adequately complement the extensive field applications.

\section{Project Objectives and Scope}

The objectives of this prnject are to identify the mechanisms by which gel treatments divert fluids in reservoirs and to establish where and how gel treatments are best applied. Several different types of gelants are being examined, including poiymer-based gelants, monomer-based gelants, and a colloidal silica gelant. This research is directed at gel applications in water injection wells, in production wells, and in high-pressure gasfloods. The work will examine how the flow properties of gels and gelling agents are influenced by permeability, lithology, and wettability. Other goals include determining the proper placement of gelants, the stability of in-place gels, and the types of gels required for the various oil recovery processes and for different scales of reservoir heterogeneity.

Several key features distinguish this research from previous work. First, results from basic reservoir engineering calculations are being used to guide the research. These calculations are being used to identify the most critical problem areas in gel technology. In contrast, a large fraction of previous research has focused on evaluating gelation chemistry, gelation kinetics, and retention of gelant components. $2,-23$ Wnile these act vities are important, out calculations $24-27$ show that the most critica! unanswered questions lie in the areas of 1) ge! placement procedurss, and 2) the dependence of gel properties on permeability, lithology, and fluid velocities. The caliulations are also valuable in that they have revealed serious deficiencies in some of the laboratory methodologies that have previously been used to evaluate gel systems. 
A second feature of this research is that it examines gel applications in production wells and in gastloods. Most previous research ${ }^{8-23}$ has been restricted to gel applications in water injection wells. Third, gel performance is being examined in carbonates as well as in sanistones. During the $1980^{\circ} \mathrm{s}, 20 \%$ of gel treatments occurred in carbonate reservoirs, ${ }^{6}$ and this percentage is growing. The pertormance of gels in carbonates is particularly relevant to gel applications in $\mathrm{CO}_{2}$ floods. A fourth distinguishing feature of the proposed research is that micromodels are being used to visualize the mechanisms by which gels divert flow of water, oil, and gas when multiple phases are present.

\section{Project Task Areas}

Eight task areas are included in this project. They are as follows:

Task 1: Equipment Design and Construction

Task 2: Screening Tests

Task 3: Gels for Producing Well Applications

Task 4: Chemical Geis in Waterflooding

Task 5: Flow Properties of Gels and Gelling Agents

Task 6: Chemical Geis in High-Pressure Gasflooding

Task 7: Mathematical Modeling

Task 8: Coordination with Other Research Programs

ill of the equipment design and construction has been completed (Task 1 ). We have acquired all equipment and techniques needed to produce both low-pressure and high-pressure micromodels. Many $(\approx 60)$ glass micromodels with various etched patterns have been produced. These are being used in several subsequent tasks, including Tasks 3, 4, and 6. Procedures for fabricating the micromodels are described in Appendix A. Video equipment for recording flow visualization studies has been procured and installed. The original design and operation of the micromodel apparatus have been described in the literature. ${ }^{28}$

A constant-temperature chamber has been constructed for performing corefloods. Three such chambers are available for use in this project. One chamber is being used to examine gel applications in production wells (Task 3 ) and in water in jection wells (Task 4). An existing chamber is being used primarily for gel applications in $\mathrm{CO}_{2}$ floods (Task 6). The apparatus that is being used during $\mathrm{CO}_{2}$ corefloods has been described earlier. ${ }^{29,30}$ In this apparatus a back-pressure regulator allows floods 10 be conducted at elevated pressures (up to $3100 \mathrm{psi}$ ). A third chamber houses the coreflood apparatus that is being used to study the rheology of gels and gelling agents in porous media.

A coreflood apparatus has been constructed for determining the rheology of gels and gelling agents in porous media. This apparatus is dedicated to studying gel performance as a function of iluid velocity in porous media (Task 5). Using this apparatus, corefloods can be conducted using the full range of flow conditions expected in a reservoir, including fluid velocities from $0.01 \mathrm{ft} / \mathrm{d}$ to 1000 $\mathrm{ft} / \mathrm{d}$ and pressure gradients from $0.1 \mathrm{psi} / \mathrm{ft}$ to $5000 \mathrm{psi} / \mathrm{ft}$. In all corefloods, at least one internal presure tap is being used to insure that observations are nor dominated by face-plugging or end eifects. This coreflood apparatus, as well as the two others described above, are interfaced with a data acquisition system and a computer.

Our gel screening siudies (Task 2) are described in Section 2 of this report. Task 3 (gels for ipplications in producing wells) is addressed in Sections 5 and 9 . Section 3 is primarily concerned with Task 4 (gels for waterflood applications). Task 5 (flow properties of gels and gelling agents) is discussed in Section 6 (and to some extent in Section 3). Preliminary studies for Task 6 (gels for 
applications in high-pressure gas floods) are presented in Section 4. Activities for Task 7 are included in Sections 3, 6, 7, 8, and 9. 


\section{GEL SCREENING STUDIES}

This section describes the results of screening tests that were performed under Task 2 of the project. These screening tests were used to establish gelation times, gel strengths, and gel stabilities for the gel systems that are being studied in other portions of the project. Several different types of gelants were examined, including a monomer-based gelant, several polymer-based gelants, and two colloidal silica gelants. By studying a variety of types of gelants, we hope 1) to compare different mechanisms of fluid diversion, and 2) to identify the types of gels that are needed for the various EOR applications (waterflood injectors, production wells, $\mathrm{CO}_{2}$ injectors, etc.)

\section{Gels Studied}

The following zels were examined:

1. A covalently crosslinked, phenolic-based gelant (Floperm $\left.325^{\otimes}\right)^{31,32}$

2. Chromium(III)-xanthan (Flocon $4800^{\otimes}$ with X-Link $\left.2000^{(3)}\right)^{33}$

3. Chromium(VI $\rightarrow$ III)-polyacrylamide (Cyanagel 100\%)

4. Chromium(III)-carboxylate-complexed polyacrylamide (MARCIT $\left.{ }^{\$}\right)^{9,34}$

5. A covalently crosslinked, vinyl-based gelant (Floperm $\left.465^{(*)}\right)^{35}$

6. Colloidal silica (Ludox $)^{36}$

Pfizer provided the Flocon 4800, X-link 2000, Floperm 325, and Floperm 465 products. American Cyanamid provided the Cyanagel 100 polyacrylamide. Marathon provided the MARCIT polymer and crosslinker. Conoco (Dupont) provided the Ludox product. Floperm 325 is a monomerbased gelant. Ludox is a colloidal silica. The other products (listings 2 through 5 above) are polymerbased gelants.

The following gel compositions were prepared and studied:

1. $6 \%$ Floperm 325 (3\% Floperm 32.5R, 3\% Floperm $325 \mathrm{~F}$ ), $0.5 \% \mathrm{KCl}$;

2. $0.4 \%$ xanthan (Flocon 4800), $73 \mathrm{ppm} \mathrm{Cr}^{3+}(\mathrm{X}$-link 2000), $100 \mathrm{ppm} \mathrm{HCHO}, 0.5 \% \mathrm{KCl}$;

3. $0.4 \%$ xanthan (Flocon 4800), $154 \mathrm{ppm} \mathrm{Cr}^{3+}(\mathrm{X}-$ ink 2000$), 100 \mathrm{ppm} \mathrm{HCHO}, 0.5 \% \mathrm{KCl}$;

4. $2.8 \%$ polyacrylamide (Cyanagel 100), $500 \mathrm{ppm} \mathrm{Na}_{2} \mathrm{Cr}_{2} \mathrm{O}_{7}, 1500 \mathrm{ppm} \mathrm{Na} \mathrm{S}_{2} \mathrm{O}_{4}, 0.5 \% \mathrm{KCl}$;

5. $\quad 0.994 \%$ polyacrylamide (MARCIT), $150 \mathrm{ppm} \mathrm{Cr}^{3+}, 1 \% \mathrm{NaCl}$;

6. $1.39 \%$ polyacrylamide (MARCIr), $212 \mathrm{ppm} \mathrm{Cr}^{3+}, 1 \% \mathrm{NaCl}$;

7. $2.5 \%$ Floperm $465 \mathrm{P}, 3 \%$ Floperm $465 \mathrm{~B}_{2}, 0.25 \%$ Floperm $4.5 \mathrm{X}, 0.5 \% \mathrm{KCl}^{\circ}$

8. $10 \%$ colloidal silica (Ludox HS-40), $2.1 \% \mathrm{NaCl}$; and

9. 10\% colloidal silica (Ludox SM), $0.7 \% \mathrm{NaCl}$.

Three criteria were uscd in selecting these compositions. First, the gel times should be long enough to allow placement in cores prior to the onset of gelation. Second, the gels should be relatively strong. Third, the gel compositions should be somewhat representative of those used in field applications. 
For most of the formulations, the brine contained $0.5 \% \mathrm{KCl}$. Different $\mathrm{NaCl}$ brines were used with the colloidal silica because the gelation process is very sensitive to salinity for these gelants. In general, salinity will not be a major variable of study in our research.

\section{Experimental Procedures}

For each of the above gelant systems, gels were prepared using mixing procedures that were specified by the supplier of the gel. Table 1 lists the $\mathrm{pH}$ of each formulation immediately after completion of the mixing procedure. Twenty milliliters of a given gelant were placed in a $50 \mathrm{ml}$ glass vial. The vials were placed in an air bath at $105^{\circ} \mathrm{F}$ for three days. The gelation time was noted (visually) during this period. Also, the gel strength was recorded after three days at $105^{\circ} \mathrm{F}$. These values are listed in Table 1. The system for assessing gel strength was taken from Ref. 9. The codes used in this system are listed in Table 2.

After three days at $105^{\circ} \mathrm{F}, 20 \mathrm{ml}$ of a $0.5 \% \mathrm{KCl}$ brine at neutral $\mathrm{pH}$ was placed on top of the $20 \mathrm{ml}$ of gel in each vial of one set of vials. In a duplicate set of vials, $20 \mathrm{ml}$ of a $0.5 \% \mathrm{KCl}$ brine at $\mathrm{pH} 3$ was placed in contact with the gel to simulate confact with brine $/ \mathrm{CO}_{2}$. In a third set of vials, $20 \mathrm{ml}$ of a $1 \% \mathrm{NaOH}$ solution $(\mathrm{pH}=12.5)$ was placed in contact with the gel to simulate conditions in alkaline floods. After brine addition, the vials were evacuated and then flame sealed. Vials were then placed in air baths or ovens at room temperature $\left(\approx 70^{\circ} \mathrm{F}\right), 105^{\circ} \mathrm{F}, 160^{\circ} \mathrm{F}$, and $200^{\circ} \mathrm{F}$. These vials were monitored regularly for gel consistency, stability, color, and other visual changes. After 30 days at a given temperature, the vials were opened and the $\mathrm{pH}$ of the free water was measured (at room temperature). Tables B1 through $B 9$ in Appendix B list important results of these experiments. These results include (1) the final code for gel strength after 30 days, (2) the final gel volume af ter 30 days relative to the initial volume of the gel, and (3) the final pH of the free water after 30 days. These results are reported as a function of temperature $\left(\approx 70^{\circ} \mathrm{F}, 105^{\circ} \mathrm{F}, 160^{\circ} \mathrm{F}\right.$, and $\left.200^{\circ} \mathrm{F}\right)$ and initial pH of the free water ( $\mathrm{pH}: 3,7$, and 12.5). In part "a" of each Table in Appendix B, both the initial gelstrength code and the final code after 30 days are indicated. For example, a listing of $I / B$ means that the initial gel-strength code was I, while the code after 30 days was B. Incidently, the assignment of a given gel-strength code can be somewhat subjective. Therefore, codes that differ by a single letter do not necessarily reflect significant differences in gel strength.

When in contact with neutral or acidic brines, many of the gels maintained most of their original gel streagth after 30 days at $200^{\circ} \mathrm{F}$. However, this does not necessarily mean that the gels will have sufficient stability for long-term applications.

Many of the gels experienced either syneresis or swelling, depending on the temperature and the $\mathrm{pH}$ of the free water in contact with the gel. In fractures, syneresis or swelling could have a significant impact on gel performance. However, in a rock matrix, the work of some researchers ${ }^{37}$ suggests that syneresis or swelling may be less important.

The $\mathrm{pH}$ of the free water in contact with the gel was reported in part "c" of each table in Appendix $B$. Changes in the $\mathrm{pH}$ of the free water reflect leaching of certain acidic or alkaline components from the gel. We note that in several cases, the pH results were somewhat unexpected. For example, consider the chromium-xanthan gels that were placed in contact with water at $\mathrm{pH}=7$ (Tables B2, B3, C2 and C3). In all or these cases, the $\mathrm{pH}$ of the brine after 30 days was lower than either the initial $\mathrm{pH}$ of the water or the $\mathrm{pH}$ of the gelant prior to gelation. Additional studies will be needed to determine why this occurred. 
TABLE 1

Summary of Gelation Results at Optimum (or Supplier-Specified) pH

Gel

Floperm 325

Xanthan (73 ppm $\mathrm{Cr}^{+}$)

Xanthan (154 ppm $\mathrm{Cr}^{3+}$ )

Cyanagel $100-\mathrm{Cr}$

MARCIT (0.994\% P.AM)

MARCIT (1.39\% PAM)

Toperm 465

Ludox HS

Ludox SM

\section{Initial}

$\mathrm{oH}$

9.0

4.2

3.9

5.0

5.8

5.8

5.1

8.25

8.25
Gel Time

(hours)

10

8.5

6

13.5

12.5

11.5

14.5

9.5

31
Initial Gel Strength (Gel Code $\left.{ }^{y}\right)$

\section{TABLE 2}

Gel Strength Codes $^{9}$

Sode

A No detectable gel formed: The gel appears to have the same viscosity as the original polymer solution and no gel is visually detectable.

B Highly flowing gel: The gel appears to be only slightly more viscous than the initial polymer solution.

C Flowing gel: Most of the obviously detectable gel flows to the vial top upon inversion.

D Moderately flowing gel: Only a small portion (about 5 to $15 \%$ ) of the gel does not readily flow to the vial top upon inversion-usually characterized as a tonguing gel (i.e., after hanging out of jar, gel can be made to flow back into bottle by slowly turning bottle upright).

E Barely flowing gel: The gel can barely flow to the vial top and/or a significant portion (>15\%) of the gel does not flow upon inversion.

F Highly deformable nonflowing gel: The gel does not flow to the vial top upon inversion.

$G$ Moderately deformable nonflowing gel: The gel flows about half way down the vial upon inversion.

H Slightly deformable nonflowing gel: The gel surface only slightly deforms upon inversion.

I Rigid gel: There is no gel-suriace deformation upon inversion.

J Ringing rigid gel: A tuning-fork-like mechanical vibration can be felt after tapping the bottle. 


\section{Gelation at Neutral pH}

For many gelants, an optimum pH exists at which the strongest gels ar formed. In the experiments described above, gelation was allowed to occur at or near the optimum pH for the particular gelant. (In all cases, the "optimum" pH was taken to be the pH specified by the supplier of the gelant.) Prior to gelation, many gelant formulations have very little buffering capacity (i.e., their $\mathrm{pH}$ can be changed significantly by adding relatively small amounts of acid or base). However, the minerals in reservoir rocks of ten have a large buffering capacity. ${ }^{38}$ Thus, even if a gelant is injected at the optimum $\mathrm{pH}$ for the gelation reaction, the reservoir minerals can quickly change the $\mathrm{pH}$ of the formulation to a more neutral value.

With this in mind, we performed a parallel set of studies where the gelant formulations were adjusted to a $\mathrm{pH}$ value of 7 at the end of the gelant mixing procedure. All other steps in the mixing procedures were identical to those specif ied by the gelant supplier. All other experimental procedures were identical to those described in the previous section, except that only free water $(0.5 \% \mathrm{KCl})$ at $\mathrm{pH} 7$ was placed in contact with the gel (i.e., after the formulation was allowed to set 3 days at $105^{\circ} \mathrm{F}$ ).

The results are tabulated in Tables $\mathrm{Cl}$ through $\mathrm{C} 9$ in Appendix $\mathrm{C}$. These results include (1) the final code For gel strength after 30 days, (2) the final gel volume after 30 days relative to the initial volume $\mathrm{O}^{-}$the gel, and $(3)$ the tinal $\mathrm{pH}$ of the free water after 30 days. These results are reported as a function of temperature $\left(\approx 70^{\circ} \mathrm{F}, 105^{\circ} \mathrm{F}, 160^{\circ} \mathrm{F}\right.$, and $\left.200^{\circ} \mathrm{F}\right)$.

Table 3 lists gelation times for the gelants at $\mathrm{pH}=7$. Also listed are the gel strengths that were measured af ter three days at $105^{\circ} \mathrm{F}$. In most cases, the gelation time at $\mathrm{pH}=7$ was less than that at the optimum (or gelant-supplier-specified) pH (compare Tables $\perp$ ard 3). In some cases, no gelation was observed. We should note that Floperm 465 has a substantial buffering capacity around $\mathrm{pH}=5$. Thus, this gelant may contain enough buffer to maintain the $\mathrm{p} H$ at a value of 5 even after injection into porous rock. Of course, a buffer could be incorporated into any gelant to have the same effect.

In several cases, the gel strength was the same after gelation at $\mathrm{pH}=7$ as that after gelation at the optimum reaction $\mathrm{pH}$. In other cases, the gel strength was significantly less af ter gelation at $\mathrm{pH}=7$.

During replicate experiments that were performed after completion of the above tests, we found some variability in the gelation results at $\mathrm{pH}=7$ for two of the gelants. For the Floperm 325 gelant, a strong, opaque gel was formed in the experiments described above. However, during replicate experiments, gelation at $\mathrm{pH}=7$ resulted in a sludge mixed with free water. Variable results were also found at $\mathrm{pH}=7$ for the chrome-xanthan gelant ( $4000 \mathrm{ppm}$ xanthan, $154 \mathrm{ppm} \mathrm{Cr}^{3+}$ ). For the experiments described in Table 3 and in Appendix $C$, a strong xanthan gel was formed. However, during some suisenuent replicate experiments, gelation was not observed at $\mathrm{pH}=7$. We are investigating the reasons for the variability of results for these two gelants. 
TABLE 3

Summary of Gelation Results at $\mathrm{pH}=7$

\begin{tabular}{|c|c|c|}
\hline Gel & $\begin{array}{l}\text { Gel Time } \\
\text { (hours) }\end{array}$ & $\begin{array}{l}\text { Initial Gel Strength } \\
\left(G^{\prime} \mid \text { Code }^{9}\right)\end{array}$ \\
\hline Floperm 325 & 6.5 & $I$ \\
\hline Xanthan (73 ppm $\mathrm{Cr}^{3+}$ ) & 7.25 & $\mathrm{E}$ \\
\hline Xanthan (154 ppm $\mathrm{Cr}^{3+}$ ) & 4.25 & I \\
\hline Cyanagel $100-\mathrm{Cr}$ & No gelation & $A$ \\
\hline $\operatorname{MARCIT}(0.994 \% \operatorname{PAM})$ & 11 & $H$ \\
\hline $\operatorname{MARCIT}(1.39 \%$ PAM) & 9.5 & I \\
\hline Floperm 465 & No gelation & A \\
\hline Ludox HS & 6.5 & $\mathrm{~J}$ \\
\hline Ludox SM & 4.5 & $\mathrm{~J}$ \\
\hline
\end{tabular}

\section{pH of Brine in Coutact With Compressed $\mathrm{CO}_{2}$}

In our studies to this point, we have presumed that brine in contact with $\mathrm{CO}_{2}$ and rock will have a $\mathrm{pH}$ value of 3 . While there is no doubt that brine in contact with $\mathrm{CO}_{2}$ alone should have a low $\mathrm{pH}$, it is conceivable that minerals in rock may buffer the $\mathrm{pH}$ at a more neutral value. We are not aware that anyone has measured the $\mathrm{pH}$ of brine in contact with both compressed $\mathrm{CO}_{2}$ and rock. This is an important issue with respect to the stability of chemicals (e.g., gels and foams) that may be used during $\mathrm{CO}_{2}$ floods. Therefore, we obtained a high-pressure $\mathrm{pH}$ probe and have measured $\mathrm{pH}$ values for brine in contact with compressed $\mathrm{CO}_{2}$ and rock (Berea sandstone, Indiana limestone, or San Andres dolomite). The results are listed in Table 4.

The pressure vessel that was used in these experiments consisted of a $6^{\prime \prime}$ length of 4130 Chrome-Moly tubing ( $1.5^{\mathrm{N}}$ inside diameter) that was fitted with 316 stainless steel end caps and a portal for the high-pressure pH probe (TBI-Bailey Controls Model \#TB567 pH sensor with Model \#540 $\mathrm{pH}$ indicator). The $\mathrm{pH}$ measurements were typically monitored over a 12 -hour period to ensure that equilibrium was reached.

The results indicate that the $\mathrm{pH}$ of a $0.5 \% \mathrm{KCl}$ brine in ccnact with $\mathrm{CO}_{2}$ at 1500 psi and $105^{\circ} \mathrm{F}$ will be in the range irom 4.4 to 5.1 . The pH at $1500 \mathrm{psi}$ is rairly insensitive to the presence or absence of rock. There are at least two unanswered questions concerning the data. First, why does brine in contact with compressed $\mathrm{CO}_{2}$ have a pH value of 4.4 to 4.8 rather than 3 ? Second, in the first set of experiments, why does the $\mathrm{pH}$ of brint without $\mathrm{CO}_{2}$ jump from 6.5 to 8.2 as the pressure is raised to $1500 \mathrm{psi}$ ? (We note that this behavior was not repeated in the replicate experiment.) These 
These experiments were intended to be cursory in order to give a quick indication of the $\mathrm{pH}$ of brine in contact with rock and $\mathrm{CO}_{2}$ at elevated pressure. A more thorough theoretical and experimental analysis may be needed. ${ }^{30}$

TABLE 4

$\mathrm{pH}$ Values of $0.5 \% \mathrm{KCl}$ Brine in Contact with Rock and Compressed $\mathrm{CO}_{2}, 105^{\circ} \mathrm{F}$ (50g. brine per $320 \mathrm{~g}$. crushed rock)

\section{Experimental Cenditions}

1. Brine only, ambient pressure

2. Brine only at $1500 \mathrm{psi}$

3. Brine and $\mathrm{CO}_{2}$ at $1500 \mathrm{psi}$

4. Brine, $\mathrm{CO}_{2}$, and crushed Berea sandstone at $1500 \mathrm{psi}$

5. Brine, $\mathrm{CO}_{2}$, and crushed Indiana limestone at $1500 \mathrm{psi}$

6. Brine, $\mathrm{CO}_{2}$, and crushed San Andres dolomite at $1500 \mathrm{psi}$

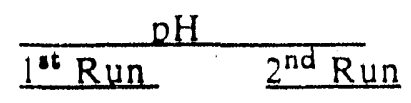

6.5

7.1

8.2

7.1

4.4

4.8

4.5

5.0

4.8

5.1

-.-

\section{Summary}

Screening tests were performed to establish gelation times, gel strengths, and gel stabilities for the gel systems that will be studied in the project, "Fluid Diversion ar.d Sweep Improvement with Chemical Gels in Oil Recovery Processes." Several different types of gelants were examined, including a monomer-based gelant, several polymer-based gelants, and two colloidal silica gelants. Gel stabilities were determined over a 30-day period as a function of temperature (room temperature to $200^{\circ} \mathrm{F}$ ) and $\mathrm{pH}$ of water in contact with the gel (water pH values of 3,7 , and 12.5). For each gelant, one set of studies was performed on gels that were allowed to form at the optimum pH for the gelation reaction (i.e., the pH specified by the gelant supplier). A second set of studies was performed on gels that were formed at $\mathrm{pH}=7$. These latter studies were initiated because reservoir rocks may force in-situ gelation reactions to occur at neutral $\mathrm{pH}$, even though the gelant was injected at some other $\mathrm{pH}$. The performance of some gels may be very different when the gel is formed at neutral $\mathrm{pH}$ rather than at the optimum gelation $\mathrm{pH}$.

Some cursory experiments were performed to determine the $\mathrm{pH}$ of brine in contact with rock and compressed $\mathrm{CO}_{2}$. The results indicate that the $\mathrm{pH}$ of a $0.5 \% \mathrm{KCl}$ brine in contact with $\mathrm{CO}_{2}$ at 1500 psiand $105^{\circ} \mathrm{F}$ will be in tne range irom 4.4 to 5.1 . This pri is tarsy insensitive to the presence or absence of rock (Berea sandstone, Indiana limestone, or San Andres dolomite). 


\section{IMPACT OF GELATION pH, ROCK PERMEABILITY, AND LITHOLOGY ON THE PERFORMANCE OF A MONOMER-BASED GEL}

ideally, gel treatments should reduce channeling of fluids through high-permeability, watered-out flow paths without damaging oil-productive zones. However, in most applications, the gelant penetrates to some extent into low-permeability, oil-productive zones. A gel treatment can either enhance or harm oil production, depending on how the gel's pertormance in low-permeability rock compares with that in the high-permeability channel. ${ }^{24-27}$

This section reports results from an experimental investigation of the effects of gelation pH, rock permeability, and lithology on the performance of a resorcinol-formaldehyde gel. This gel was chosen for study because its placement in porous media is not complicated by some of the factors that influence placement of polymeric gelants. In particular, prior to gelation, aqueous resorcinolformaldehyde solutions 1) are Newtonian, 2) exhibit nearly the same viscosity as water., and 3) can readily propagate through sandstone and carbonate porous media without experiencing any significant chemical retention. In contrast, polymeric gelants usually are viscous and non-Newtonian. More important, the components of polymeric gelants (i.e., polymers and metallic crosslinkers) exhibit permeability - and lithology-dependent retention. $40-43$ Insights obtained by studying this relatively simple resorcinol-formaldehyde gel may be valuable when assessing the performance of more complex gels in fluid diversion.

Resorcinol and formaldehyde (see Fig. 1) are small molecules that are very solu'le in water. Resorcinol and formaldehyde will polymerize to form gels, as shown in Fig. 2. These reactions are - ery similar to those that occur during the formation of phenol-formaldehyde resins (e.8., Bakelite $).{ }^{44}$ This class of gels has been used for fluid diversion in field applications. ${ }^{31,32,45,46}$

Several terms should be defined for the reader's benefit. The terms "gelant" and "gelling agent ${ }^{n}$ here refer to the liquid formulation prior to gelation. Resistance factor, $F_{r}$, is defined as water mobility divided by mobility of the gelant. It is equivalent to the effective viscosity of the gelant in porous media relative to that of water. Residual resistance factor, $F_{m}$, is defined as water mobility in the absence of gel divided by water mobility in the presence of gel. Residual resistance factor is a measure of the permeability reduction caused by gel.

\section{pH Depeadence of Gelation}

All gelant formulations throughout this study contained $3 \%$ (by weight) resorcinol $(0.27 \mathrm{M})$, 3 * formaldehyde $(1 \mathrm{M})$, and $0.5 \% \mathrm{KCl}(0.067 \mathrm{M})$. All chemicals used were reagent grade. Also, all core experiments and gelation studies were performed at $105^{\circ} \mathrm{F}\left(41^{\circ} \mathrm{C}\right)$.

The product formed by the reaction of resorcinol with formaldehyde depends on pH. At $\mathrm{pH}=9$, a strong, ringing gel is formed that is clear and red. No free water remains after gelation. However, as the initial pH is decreased, gel formation becomes less perfect. With an initial pH of 7 , an opaque orange-white gel is formed, and some free water remains after the reaction. The gelation time (at $105^{\circ} \mathrm{F}$ ) is about four hours at $\mathrm{pH}=9$ and is five to seven hours when the initial $\mathrm{pH}$ is 7 . As initial pH value is decreased below 7 , the gelation time increases, and the final ratio of free water $t$ gel also increases.

As the reaction berween resorcinol and formaldehyde procesds, the pHtends to decrease. For example, for a gelant at an initial pH of 7 , the pH gradually declines 10 a value of 5.1 over the course of five hours. Under similar conditions, the $\mathrm{pH}$ remains stable ar a value of 7 for solutions of resorcinol without formaldehyde or of formaldehyde without resorcinol. 
<smiles>Oc1ccccc1</smiles>

RESORCINOL

$\mathrm{C}_{6} \mathrm{H}_{4}(\mathrm{OH})_{2}$

$M_{w}=110$<smiles>C=O</smiles>

FORMALDEHYDE $\mathrm{HCHO}$

$M_{w}=30$

Fig. 1. Compositions of Resorcinol and Formaldehyde.

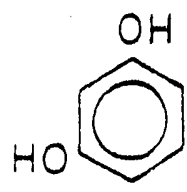

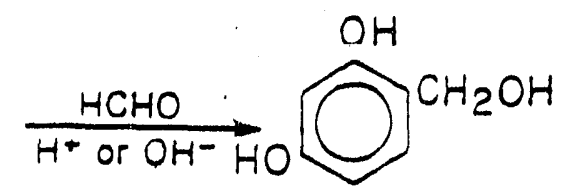

$\frac{\mathrm{C}_{6} \mathrm{H}_{4}(\mathrm{OH})_{2}}{\mathrm{HO}_{\mathrm{O}}} \stackrel{\mathrm{OH}}{\mathrm{CH}_{2}} \longrightarrow \mathrm{OH}$
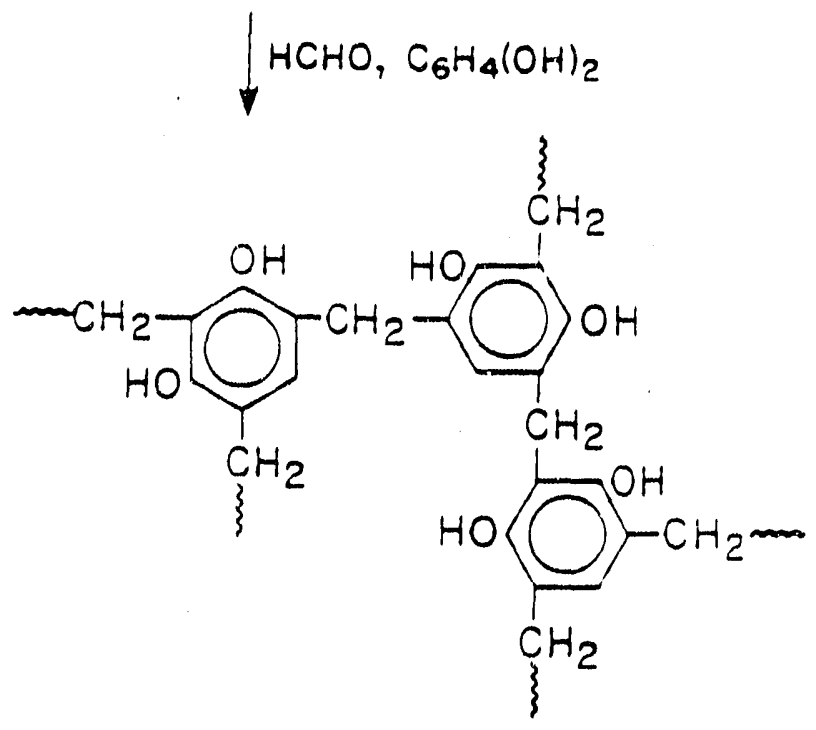

Fig. 2. Reaction of Resorcinol with Formaldehyde. 
Resorcinol and formaldehyde have very little capacity to buffer the $\mathrm{pH}$ of a solution. This is shown in Fig. 3. Thus, acid generated during the resorcinol-formaldehyde reaction can decrease $\mathrm{pH}$ and affect the final nature of the product. Since gelation is sensitive to $\mathrm{pH}$, all factors that affect the $\mathrm{pH}$ should be of concern when determining the nature and the performance of the gel. In addition to the reaction itself, several rock minerals can have an important influence on solution $\mathrm{pH}$. For example, clay minerals can reversibly exchange metallic cations (e.g. $\mathrm{Na}^{+}, \mathrm{Ca}^{2+}$ ) with hydrogen ions in solution. ${ }^{38}$ Clay minerals can also react irreversibly with hydrogen or hydroxide ions. ${ }^{4 "}$ Dissolution and precipitation of minerals can also change $\mathrm{pH}^{48-50}$ This raises a concern that the reaction product formed in beaker tests may differ from those formed in porous media. Furthermore, during laboratory corefloods with unbuffered gelants, a gradient of $\mathrm{pH}$ values may exist in the core. Since the nature and performance of gels varies considerably with $\mathrm{pH}$, coreflood results using unbuffered gelants may be difficult to scale to field applications.

With this in mind, we have examined the use of buffers to maintain constant $p H$ values. A phosphate buffer was found to be effective at maintaining $\mathrm{pH}$ at 7 ; an acetate buffer was used at $\mathrm{pH}=5$; and carbonate buffers were used at $\mathrm{pH}$ values of 6 and 9 . In all cases, the buffer concentration was $0.05 \mathrm{M}$. With a sodium bicarbonate buffer at $\mathrm{pH}=9$, the gelation time, gel strength, and appearance were identical to those without the carbonate buffer at $\mathrm{pH}=9$.

At an initial $\mathrm{pH}$ of 7 , the gelation time was five to seven hours either with or without the phosphate buffer. Also, the gel product had the same color (opaque orange-white) with or without phosphate. With phosphate, no free water remained after gelation, and the gel had a smooth, solid appearance. However, without the phosphate buffer, free water remained after the reaction, and the "gel" appeared as a grainy precipitate. Without phosphate, the final ratio of free water to "gel" ranged from $5: 1$ to $1: 10$ during several replicate experiments. Thus, there is variability that is not currently understood. In contrast, for cases where buffers were used, results were reproducible. This provides another argument in favor of using buffers during laboratory experiments.

For gelants buffered with bicarbonate at $\mathrm{pH}=6$, no free water formed, but the gel was more grainy in appearance than that with phosphate at $\mathrm{pH}=7$. For gelants buffered with acetate at $\mathrm{pH}=5$, free water in contact with a grainy precipitate was formed (ratio of $\approx 3: 1$, respectively). We cannot eliminato the possibility that the buffers interfere $w$ ith the resorcinol-formaldehyde reaction by some means other than by affecting $\mathrm{pH}$. However, we suspect that $\mathrm{pH}$ alteration is the dominant factor.

We also determined the inherent permeability of gel formed at $\mathrm{pH}=9$. Gel was allowed to form in a glass "micromodel" that had internal dimensions of $10.35 \mathrm{~cm} \times 0.21 \mathrm{~cm} \times 0.0178 \mathrm{~cm}$. Before placing the gel, the effective "permeability" of the micromodel was $893 \mathrm{D}$. After allowing the gel to form, the permeability to brine was found to be $6.2 \mu \mathrm{D}$.

\section{Chemical Transport in Porous Media Prior to Gelation}

For an aqueous solution that contains $3 \%$ resorcinol, $3 \%$ formaldehyde, and $0.5 \% \mathrm{~K} \mathrm{Cl}$, the viscosity at $105^{\circ} \mathrm{F}$ (prior to gelation) is $0.75 \mathrm{cp}$-nearly the same as that of a brine that contains $0.5 \%$ $\mathrm{KCl}(0.65 \mathrm{cp})$. During flow through cores, all resistance factors for resorcinol-formaldehyde solutions (again, prior to gelation) were cbserved to be near one. This was noted in both sandstone and carbonate cores having permeabilities ranging from 7 to $700 \mathrm{md}$.

Previous work has demonstrated that propagation of formaldehyce is not retarded during flow inrough reservoir rock. ${ }^{51}$ In our work, corefloods were performed to assess retention of resorcinol in Berea. During injeciion of a continuous bank of $3 \%$ resorcinol to displace brine from $288-\mathrm{md}^{3}$ Berea, the 50\%-concentration level for resorcinol in the effluent (monitored spectrophotometrically) 


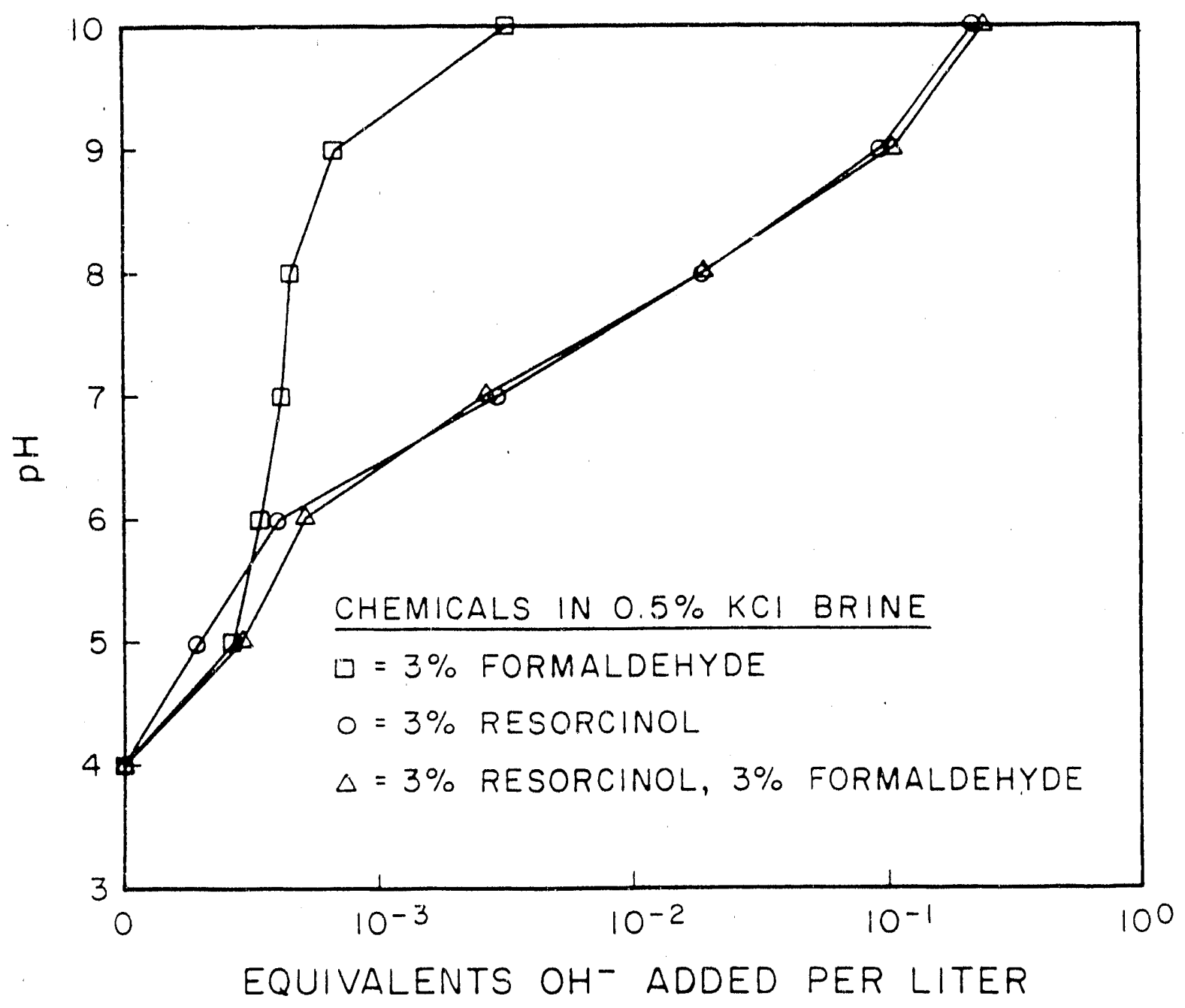

Fig. 3. Bufier Cápacicies of Resorcinol and Formaldehyde. 
indicated that resorcinol was not adsorbed or retained by the porous media to any significant extent.

\section{Coreflood Procedures}

In each of the corefloods performed during this work, the porosity and permeability to brine vere first determined. All cores were about $15-\mathrm{cm}$ long and $3.6 \mathrm{~cm}$ in diameter. All cores had one internal pressure tap located $2.5 \mathrm{~cm}$ from the inlet rock face. The cores were not fired. Tracer studies were performed to determine the dispersivity of the core and to confirm the pore volume determination. These studies involved injecting a brine bank that contained potassium iodide as a tracer. The tracer concentration in the effluent was monitored spectrophotometrically at a wavelength of $230 \mathrm{~nm}$.

"Then, three pore volumes of resorcinol-formaldehyde gelant were injected using a flux of 15.7 $\mathrm{it} / \mathrm{d}$. Resistance factors were monitored in the two core sections during this entire time. We also continuously monitored pH values in the effluent. Effluent samples were coliected and monitored 10 determine whether the gelation characteristics of the effluent differed from ihose of gelant that had not been injected. After injection of the gelant, cores were shut-in for three to four days (at $\left.105^{\circ} \mathrm{F}\right)$.

After shut-in, brine was injected to determine residual resistance factors $\left(\mathrm{F}_{\mathrm{rr}}\right)$. Low injection rates were used first. The $F_{r r}$ values reported here were measured using the second segment $(\approx 12.5$ $\mathrm{cm}$ ) of the core. Note was made of how rapidly $F_{\text {r }}$ values stabilized and whether any gel was forced from the core along with the effluent. After stabilization, brine injection rates were increased, and the observations were repeated. Then, the injection rate was decreased to determine whether $F_{\mathrm{rr}}$ values at lower rates had changed. This process was repeated with successively higher rates. The objectives of this procedure were (1) to determine whether gel mobilization occurred at a given flow rate, and (2) to determine the apparent rheology of the gel in porous media.

After the $F_{r r}$ values had been determined, additional tracer studies were performed to determine (1) the final pore volume that was occupied by the gel, and (2) the final dispersivity of the cure.

\section{Permeability Reduction After Gelation}

In each of the core experiments, residual resistance factors were determined over a range of fluid velocities. In many cases, $F_{\mathrm{rr}}$ values decreased significancly upon exposure to successively higher brine flow rates. Table 5 lists $F_{r r}$ data for a resorcinol-formaldehyde gel (that was buffered at $\mathrm{pH}=7$ ) in 63 -md Berea sandstone. Residual resi. tance factors decreased from 1735 after first exposure to a fluid flux of $0.025 \mathrm{ft} / \mathrm{d}(76.2 \mathrm{psi} / \mathrm{ft})$ to 1120 after exposure to a flux of $0.393 \mathrm{ft} / \mathrm{d}(773$ $\mathrm{psi} / \mathrm{ft})$. However, when flow rates were subsequently reduced, the $F_{r r}$ values remained fairly constant. The results suggest that upon first exposure to a giver fluid velocity, a certain amount of gel breaks down to allow a flow path through the porous medium. Flow of brine through this porous medium then appears more or less Newtonian until the previous maximum in fluid velocity is exceeded. 
TABLE 5

Results from Brine Injection After Gel Placement in 63-md Berea Sandstone

(Gelant contains $3 \%$ resorcinol, $3 \%$ formaldehyde, $0.5 \% \mathrm{KCl}, 0.05 \mathrm{M}$ phosphate, $\mathrm{pH}=7$ ) $\phi=0.192, \mu_{w}=0.70 \mathrm{cp}, 105^{\circ} \mathrm{F}$

\begin{tabular}{|c|c|c|c|c|}
\hline $\begin{array}{c}\text { Flux } \\
(\text { ft } / d)\end{array}$ & $\begin{array}{l}\text { Pressure } \\
\text { Gradient } \\
\text { (osi/ft) }\end{array}$ & $\begin{array}{l}\text { Final } \\
1 \\
\text { (md) }\end{array}$ & $\mathrm{F}_{\mathrm{rr}}$ & \\
\hline 0.025 & 76.2 & 0.0363 & 1735 & \\
\hline $\begin{array}{l}0.050 \\
0.025\end{array}$ & $\begin{array}{r}140.5 \\
71.8\end{array}$ & $\begin{array}{l}0.0394 \\
0.0385\end{array}$ & $\begin{array}{l}1600 \\
1635\end{array}$ & $\bar{F}_{r r}=1620$ \\
\hline $\begin{array}{l}0.100 \\
0.025\end{array}$ & $\begin{array}{l}259 \\
68.5\end{array}$ & $\begin{array}{l}0.0427 \\
0.0404\end{array}$ & $\begin{array}{l}1475 \\
1560\end{array}$ & $\overline{\mathrm{F}}_{\mathrm{rr}}=1520$ \\
\hline $\begin{array}{l}0.201 \\
0.100 \\
0.025\end{array}$ & $\begin{array}{r}494 \\
267 \\
58.4\end{array}$ & $\begin{array}{l}0.0450 \\
0.0414 \\
0.0474\end{array}$ & $\begin{array}{l}1400 \\
1520 \\
1330\end{array}$ & $\bar{F}_{\pi}=1420$ \\
\hline $\begin{array}{l}0.393 \\
0.202 \\
0.100 \\
0.025\end{array}$ & $\begin{array}{c}773 \\
415 \\
211 \\
48.3\end{array}$ & $\begin{array}{l}0.0563 \\
0.0538 \\
0.0525 \\
0.0573\end{array}$ & $\begin{array}{l}1120 \\
1170 \\
1200 \\
1100\end{array}$ & $\bar{F}_{\pi}=1150$ \\
\hline
\end{tabular}

Table 6 provides another example for a gel that was buffered at $p H=9$ in $49-m$ merea sandstone. With this gel, $F_{\pi}$ values experience a more dramatic decrease upon exposure to successively higher injection rates. This is also shown in Fig. 4. Upon subsequent reduction of injection rates, a mild shear-thinning character is observed for the gel at $\mathrm{pH}=9$. Equations relating $F_{\pi}$ values to flux values ( $u$, in $\mathrm{ft} / \mathrm{d}$ ) are included in Table 6 . Similar data from other core experiments are listed in Tables D1-D10 in Appendix D. (These tables list data in the order in which they were collected.) A summary of this data is included in Table 7 . In determining each $F_{r r}$ relation in Table 7, the core was first exposed to an injection rate that resulted in the maximum pressure gradient specified. Then, $\mathrm{F}_{\mathrm{r}}$ values were determined at a number of lower rates.

The coefficient of flux in the $F_{\pi}$ relations in Table 7 provides a means for comparing $F_{\pi}$ values at a fixed flux $(1 \mathrm{ft} / \mathrm{d})$. For a given lithology and permeability, the highest $F_{m}$ values were observed for gels formed at $\mathrm{pH}=9$. This was anticipated since the most rigid gels are formed at $\mathrm{pH}=9$ during beaker tests.

For gels that were buffered at $p H$ values of 7 or sbove, $F_{I T}$ values were very high. In fact, these values are so high that rock matrix treated by these gels would be effectively plugged. Thus, when placing these gels in a reservoir, caution should be used to prevent damage to oil-productive zones.

For gels that were buffered at pH values of 6 or less, $F_{m}$ values were near one-indicating that the "gel" had little or no eifect. Thus, there is a fairly narrow range of $\rho H(6$ to 7$)$ over which $F_{m}$ values will change from unity to very high values. 
For gels that were buffered at a given $\mathrm{pH}$ in Berea sandstone, $\mathrm{F}_{\mathrm{rr}}$ values generally remained about the same or increased with decreasing permeability. (Results at $\mathrm{pH}=6.5$ appear to provide an exception. However, this may be an artifact since different buffers were used and since the $F_{r r}$ values were very sensitive to $\mathrm{pH}$ in this $r$ gion.) For some of the data observed in limestone cores, $F_{r r}$ values were less than those in more-permeable Berea cores. Thus, $F_{r r}$ values can be affected by both permeability and lithology.

For gels that were injected at $\mathrm{pH}=7$ but were not buffered, we are less certain about the actual $\mathrm{pH}$ at which the gelation reaction occurred. From the $\mathrm{F}_{\mathrm{rr}}$ values, we suspect that reaction $\mathrm{pH}$ values were between 6 and 7 . However, it is quite possible that the reaction $\mathrm{pH}$ values were different in the three unbuffered cases-especially in the sandstone cores vs. the carbonate core.

Tracer studies provide interesting insights about the fraction of the total pore volume that was occupied by gel. In Table $7, \mathrm{~V}_{\mathrm{pf}} / \mathrm{V}_{\mathrm{pi}}$ refers to the fraction of the original pore volume that was sampled by the iodide tracer af ter gel placement (as determined by the $50 \%$ tracer-concentration level in the effluent). For gels at $\mathrm{pH}=9$, the gel apparently occupied $87 \%$ to $99 \%$ of the pore space. Generally, as the gelation pH was reduced, a smaller fraction of the pore volume was occupied by the gel. In one case (unbuffered gel at $\mathrm{pH}=7$ in $57-$ md Berea), the gel reduced permeability by a factor if 128 , apparently without reducing the pore volume. In other cases (gel buffered at $\mathrm{pH}=7$ in $63-\mathrm{md}$ Berea and in 7-md limestone), large $F_{r r}$ values were associated with fairly small reductions in pore volume (27\% to $34 \%)$. One could speculate how a small volume of gel could cause large permeability reductions. Perhaps, small gel particles lodge in pore throats-thereby, dramatically reducing brine pernieability without occupying much volume.

Table 7 also $h$ s: dispersivity results obtained during tracer studies. The quantity $\alpha_{f} / \alpha_{i}$ refers to the final dispersivity turing tracer injection after gelation divided by the initial dispersivity value before gel placement. The effluent tracer curves usually fit quite well using the error-function solution. ${ }^{52}$ Figures 5-7 show several tracer curves that were obtained before and after gel placement.

TABLE 6

Resu, ts From Brine Injection After Gel Placement in 49-md Berea Sandstone (Gelant contains 3\% resorcinol, 3\% formaldehyde, $0.5 \% \mathrm{KCl}, 0.05 \mathrm{M} \mathrm{NaHCO}_{3}, \mathrm{pH}=9$ ) $\phi=0.200, \mu_{w}=0.67 \mathrm{cp}, 105^{\circ} \mathrm{F}$

\begin{tabular}{|c|c|c|c|c|}
\hline $\begin{array}{r}\text { Flux } \\
(\mathrm{ft} / \mathrm{d}) \\
\end{array}$ & $\begin{array}{l}\text { Pressure } \\
\text { Gradient } \\
\text { (psi/ft) }\end{array}$ & $\begin{array}{l}\text { Final } \\
k \\
\text { (md) }\end{array}$ & $F_{r r}$ & \\
\hline 0.025 & 466 & 0.0057 & 8600 & \\
\hline $\begin{array}{l}0.050 \\
0.025\end{array}$ & $\begin{array}{l}471 \\
243\end{array}$ & $\begin{array}{l}0.0113 \\
0.0109\end{array}$ & $\begin{array}{l}4350 \\
4490\end{array}$ & $F_{r r}=3793 u^{-0.06}$ \\
\hline $\begin{array}{l}0.126 \\
0.025 \\
0.050\end{array}$ & $\begin{array}{l}622 \\
156 \\
313\end{array}$ & $\begin{array}{l}0.0214 \\
0.0170 \\
0.0184\end{array}$ & $\begin{array}{l}2280 \\
2890 \\
2660\end{array}$ & $F_{\mathrm{rr}}=1688 \mathrm{u}^{-0.16}$ \\
\hline $\begin{array}{l}0.251 \\
0.025\end{array}$ & $\begin{array}{l}690 \\
100\end{array}$ & $\begin{array}{l}0.0385 \\
0.0266\end{array}$ & $\begin{array}{l}1270 \\
1840\end{array}$ & $F_{r r}=1017 u^{-0.16}$ \\
\hline $\begin{array}{l}0.628 \\
0.12 i \\
0.025\end{array}$ & $\begin{array}{r}748 \\
199 \\
50\end{array}$ & $\begin{array}{l}0.0889 \\
0.0670 \\
0.0531\end{array}$ & $\begin{array}{l}550 \\
730 \\
920\end{array}$ & $F_{r r}=515 \mathrm{u}^{-0.16}$ \\
\hline
\end{tabular}




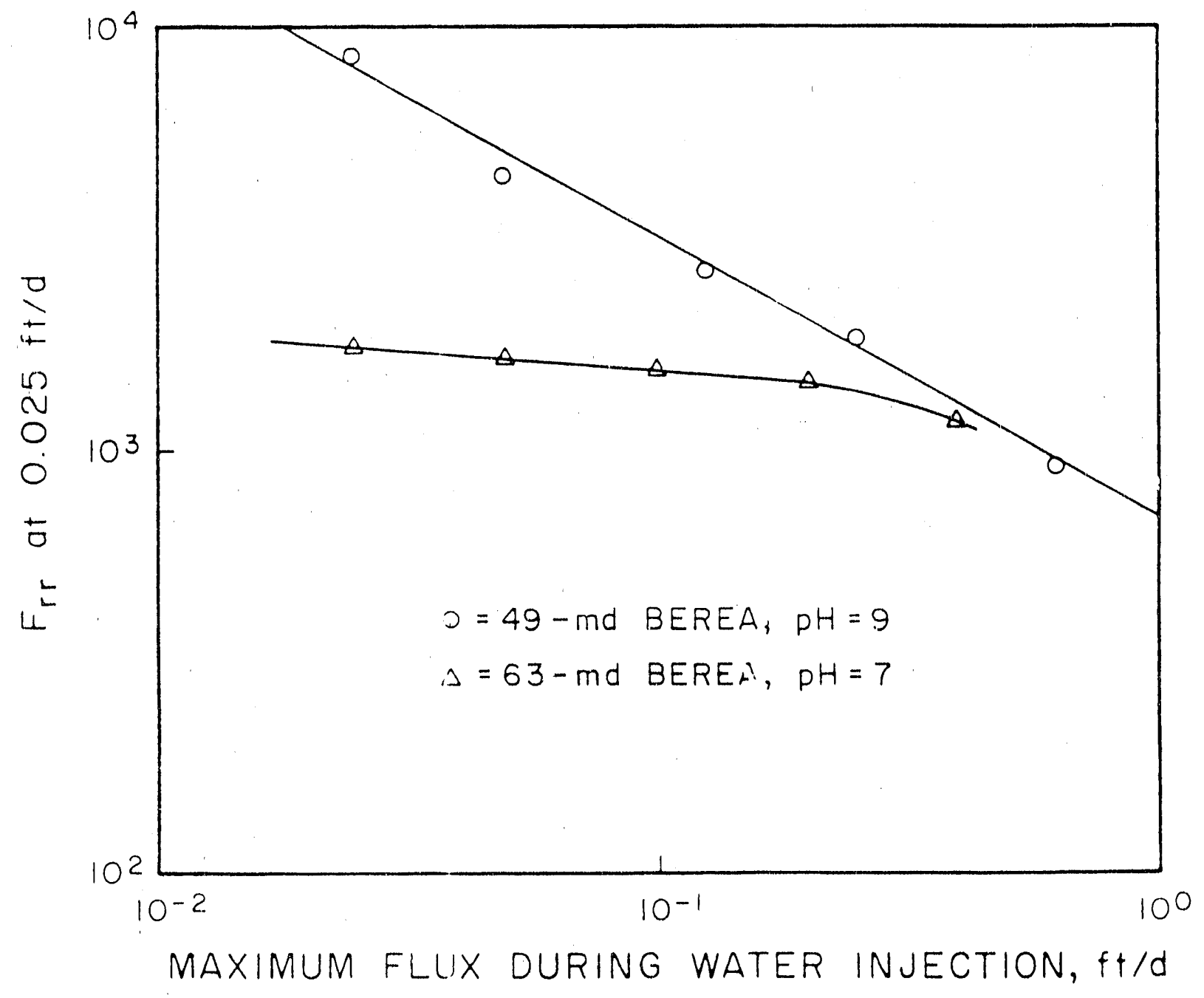

Fig, 4. F Value at $0.025 \mathrm{ft} / \mathrm{d}$ After Injecting Brine at the Maximum Flix Specified on the Abscissa. 
TABLE 7

Summary of Results From Brine Injection After Gelation (Gelant contains $3 \%$ Resorcinol, $3 \%$ Formaldehyde, $0.5 \% \mathrm{KCl}, 105^{\circ} \mathrm{F}$ )

\begin{tabular}{|c|c|c|c|c|c|c|}
\hline Rock & $\mathrm{pH}$ & Buffer & $E_{r}$ Relation & $\begin{array}{l}\text { Maximum } \\
\mathrm{dp} / \mathrm{dl} \\
(\mathrm{psi} / \mathrm{ft})\end{array}$ & $\underline{V}_{p f}\left\langle V_{p i}\right.$ & $\underline{\alpha}_{\mathrm{f}}<\alpha_{i}$ \\
\hline 570-md BS & 9 & $0.05 \mathrm{M} \mathrm{NaHCO}_{3}$ & $\begin{array}{l}F_{r r}=2170 \\
F_{r r}=1250\end{array}$ & $\begin{array}{l}25 ! \\
750\end{array}$ & 0.095 & 106 \\
\hline $49-$ md BS & 9 & $0.05 \mathrm{M} \mathrm{NaHCO}_{3}$ & $\begin{array}{l}F_{r r}=3793 u^{-0.06} \\
F_{r r}=1688 u^{-0.15} \\
F_{r r}=1017 u^{-0.16} \\
F_{r r}=515 u^{-0.16}\end{array}$ & $\begin{array}{l}471 \\
622 \\
690 \\
748\end{array}$ & 0.13 & 11.5 \\
\hline 7.4-md LS & 9 & $0.05 \mathrm{M} \mathrm{NaHCO}_{3}$ & $F_{r r}=1594 u^{-0.16}$ & 1006 & 0.01 & 2.9 \\
\hline $455-\mathrm{md} \mathrm{BS}$ & 7 & $0.05 \mathrm{M}$ phosphate & $\begin{array}{l}F_{r r}=970 u^{-0.11} \\
F_{r r}=662 u^{-0.19} \\
F_{r r}=500 u^{-0.17} \\
F_{r r}=386 u^{-0.10} \\
F_{r r}=286\end{array}$ & $\begin{array}{r}56 \\
74 \\
100 \\
147 \\
1103\end{array}$ & 0.12 & 5.2 \\
\hline 63-md BS & 7 & $0.05 \mathrm{M}$ phosphate & $\begin{array}{l}F_{r r}=1735 \\
F_{r r}=1620 \\
F_{r r}=1520 \\
F_{r r}=1420 \\
F_{r r}=1150\end{array}$ & $\begin{array}{r}76 \\
141 \\
259 \\
494 \\
773\end{array}$ & 0.73 & 94 \\
\hline 7-md LS & 7 & $0.05 \mathrm{M}$ phosphate & $\begin{array}{l}F_{r r}=404 \\
F_{r r}=365 \\
F_{r r}=355 \\
F_{r r}=326\end{array}$ & $\begin{array}{l}160 \\
283 \\
534 \\
910\end{array}$ & 0.66 & 4.4 \\
\hline $390-\mathrm{md} \mathrm{BS}$ & 7 & none & $F_{\mathrm{rr}}=83.1 \mathrm{u}^{-0.05}$ & 298 & 0.13 & 64 \\
\hline $57-\mathrm{md} \mathrm{BS}$ & 7 & none & $F_{r r}=128$ & $160-2130$ & 1.0 & 29 \\
\hline $13-m d$ LS & 7 & none & $F_{\mathrm{rr}}=4.7 \mathrm{u}^{-0.04}$ & $24-504$ & 0.98 & 0.35 \\
\hline 28-md BS & 6.5 & $0.05 \mathrm{M}$ phosphate & $F_{\mathrm{rr}}=24.4 \mathrm{u}^{-0.26}$ & $8-930$ & 0.39 & 9.0 \\
\hline $288-\mathrm{md} \mathrm{BS}$ & 6.5 & $0.05 \mathrm{M} \mathrm{NaHCO}_{3}$ & $\begin{array}{l}F_{r r}=417 u^{-0.28} \\
F_{r r}=404 u^{-0.19} \\
F_{r r}=242 u^{-0.31} \\
F_{r r}=248 \\
F_{r r}=153 \\
F_{r r}=123\end{array}$ & $\begin{array}{r}29 \\
41 \\
48 \\
69 \\
169 \\
629\end{array}$ & 0.99 & 1.5 \\
\hline 7.4-md LS & 6.5 & $0.05 \mathrm{M} \mathrm{NaHCO}_{3}$ & $F_{r r}=1.5 u^{-0.07}$ & $1-29$ & 0.99 & 1.5 \\
\hline
\end{tabular}


TABLE 7 (cont.)

\begin{tabular}{|c|c|c|c|c|c|c|}
\hline Rock & $\mathrm{pH}$ & Buffer & $E_{r}$ Relatisn & $\begin{array}{l}\text { Maximum } \\
\mathrm{dp} / \mathrm{dl} \\
(\mathrm{psi} / \mathrm{ft})\end{array}$ & $\underline{V}_{p r} / V_{p i}$ & $\underline{\alpha}_{r}<\alpha_{i}$ \\
\hline 704-md BS & 6 & $0.05 \mathrm{M} \mathrm{NaHCO}_{3}$ & $F_{\mathrm{rr}}=1.8$ & $1-8$ & 0.87 & 5.1 \\
\hline $61-m d B S$ & 6 & $0.05 \mathrm{M} \mathrm{NaHCO}_{3}$ & $F_{r r}=2.1 u^{-0.14}$ & $5-41$ & 1.0 & 0.97 \\
\hline $77-\mathrm{md} \mathrm{BS}$ & 6 & $0.05 \mathrm{M}$ phosphate & $F_{\mathrm{rr}}=1.3$ & $3-27$ & 0.93 & 1.4 \\
\hline 573-md BS & 5 & $0.05 \mathrm{M}$ acetate & $F_{r r}=1.0$ & $2-5$ & 0.97 & 1.35 \\
\hline
\end{tabular}

Dispersivity values for cores before exposure to gel were roughly the same in highpermeability Berea as in low-permeability Berea $(\approx 0.1 \mathrm{~cm})$. However, dispersivity values for Indiana limestone were typically five to ten times greater than those for Berea.

In most cases, the presence of gel increased dispersivity. At pH values above 6 , dispersivity values in Berea were 5 to 106 times greater after gel placement than before gel placement. Gelinduced dispersivity changes in Indiana limestone were generally less than those in Berea sandstone.

When the tracer studies were performed at very low injection rates, tracer curves sometimes showed signs of an exchange of iodide between the gel and the mobile brine. We observed that the degree of "tailing" exhibited by the tracer curve increased with decreasing injection rate. As injection rate decreased, there was a greater need to use a capacitance model (e.g., a "Coats-Smith" model ${ }^{53}$ ) to describe the tracer data. Further evidence that iodide was exchanging between the gel and the brine was found when brine was used to flush tracer from the core. After injecting many pore volumes of brine at a high rate to displace tracer, no iodide was detected in the effluent. However, if the core was shut-in for a day, and then additional brine was injected, iodide was detected in the iirst pore volume of effluent.

\section{Exploiting pH to Optimize Gel Placement}

We have shown that residual resistance factors provided by a resorcinol-formaldehyde gel depend on the $\mathrm{pH}$ at which gelation occurs. This is especially true over the $\mathrm{pH}$ range from 6 to 7 . For gelation $\mathrm{pH}$ values between 6 and 7, residual resistance factors can change from one to more than 1000.

Can this pH dependence of gelarion be exploited to optimize gel placement? In concept, ion exchange and other reactions with rock ininerals could retard lide niovecnen: of a pH front to a greater extent in one zone than in another zone. Perhaps different rates of propagation of pH fronts could be exploited to attain high $F_{r r}$ values in the most-permeable zones but low $F_{r r}$ values in lesspermeable zones. 


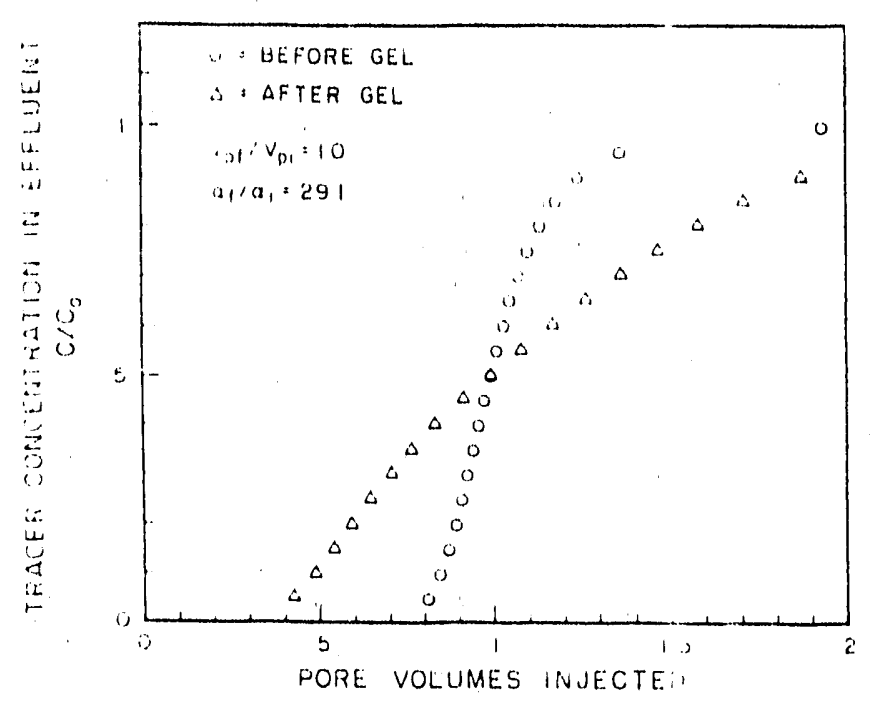

Fig. 5. Tracer Results during Brine Injection Before vs. After Gel Placement in 57-md Berea. No buffer. Initial Gelant $\mathrm{pH}=7$.

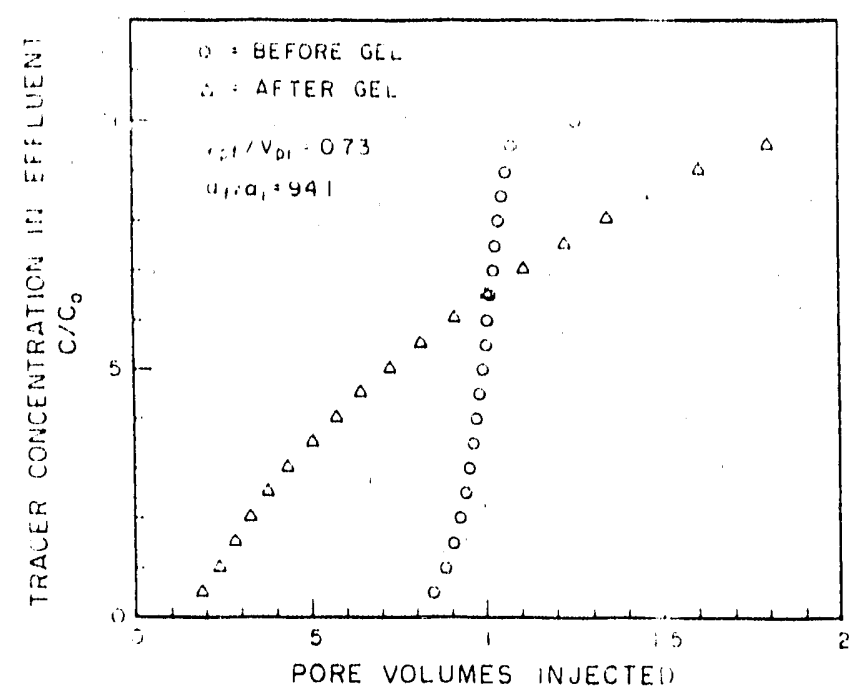

Fig. 6. Tracer Results during Brine Injection Before vs. After Gel Placement in 63-md Berea. Phosphate buffer. Gelant $\mathrm{pH}=7$.

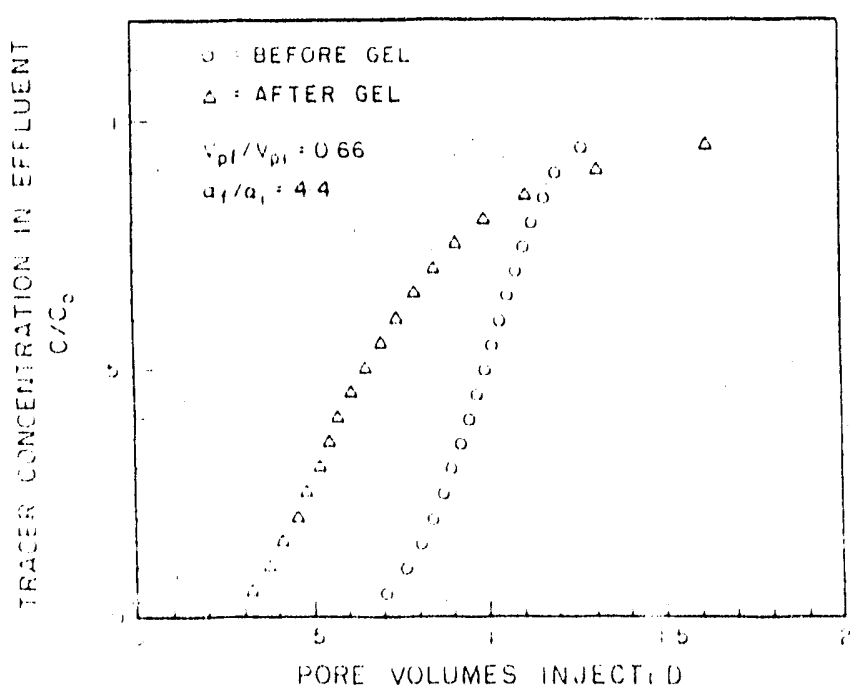

Fig. 7. Tracer Results during Brine Injection Before vs. After Gel Placement in 7-md Limestone. Phosphate buffer. Gelant $\mathrm{pH}=7$. 
Previous researchers have described the propagation of buffered and unbuffered fluid banks through porous media. ${ }^{38,54}$ As with any species, the propagation of $\mathrm{H}^{+}$or $\mathrm{OH}^{-}$through porous media depends on (1) the injected concentration of the species, (2) the volume of fluid injected, (3) the number of sites available for adsorption or exchange, and (4) any reactions or equilibria that involve the species.

A parameter, $a_{r}$ is defined here as the number of pore volumes of a chemical formulation that must be injected to satisfy all available retentive sites in one pore volume of the porous medium. Eq. I can be used to evaluate $a_{r}$.

$$
a_{r}=\frac{n_{\text {rock }}}{n_{\text {sol }}}
$$

Here, $\mathrm{n}_{\text {mok }}$ is the amount of a specific chemical that is removed by the rock from a iertain pore volume of fluid under a particular set of conditions. The parameter, $\mathrm{n}_{\mathrm{sol}}$, is the amount of the species of interest in solution per unit of volume of the injected formulation. When considering changes in $\mathrm{H}^{+}$or $\mathrm{OH}^{-}$concentrations, both $\mathrm{n}_{\text {rock }}$ and $\mathrm{n}_{\text {sol }}$ can be expressed in units of equivalents per liter (eq/1) of pore volume.

The capacity of a given rock to retard a pH front is related to the quantities and specific types of clays and other minerals that are present. The ion exchange capacity $\left(n_{\max }\right)$ provides a measure of the number of sites that can reversibly exchange cations or hydrogen ions. Berea, which is considered a relatively clean sandstone, has an ion-exchange capacity around 5 milli-equivalents per kilogram (meq $/ \mathrm{kg}$ ) of rock. ${ }^{55}$ For reservoir rocks, ion-exchange capacities from 4 to $70 \mathrm{meq} / \mathrm{kg}$ have been reported. 55

The maximum value for $n_{\text {rock }}$ that is due to ion exchange is given by Eq. $2 .^{38}$

$$
\mathrm{n}_{\text {rock }}=\frac{\mathrm{n}_{\max \rho_{\mathrm{r}}(1-\phi)}}{\phi}
$$

For a sandstone with a porc ity $(\phi)$ of 0.2 , a rock density $\left(\rho_{\mathrm{r}}\right)$ of $2.65 \mathrm{~g} / \mathrm{cm}^{2}$, and an ion-exchange capacity of $10 \mathrm{meq} / \mathrm{kg}$, the value of $\mathrm{n}_{\text {rock }}$ is given by Eq. 3 .

$$
\mathrm{n}_{\mathrm{rock}}=\frac{\left(10^{-2} \mathrm{eq} / \mathrm{kg}\right)\left(2.65 \mathrm{~g} / \mathrm{cm}^{3}\right)\left(10^{-3} \mathrm{~kg} / \mathrm{g}\right)\left(10^{3} \mathrm{~cm}^{3} / 1\right)(1-0.2)}{0.2}=0.106 \mathrm{eq} / 1
$$

Eqs. 2 and 3 assume that retention of the species is independent of concentration and that no other reactions involve the species (othe: than irreversible retention). However, adsorption of the species of ten will be governed by a Langmuir-type isotherm ${ }^{38}$ so that $n_{\text {rock }}$ wili be less than the value given by Eq. 2 .

Concerning $\mathrm{n}_{30 j}$, equilibria may exist between components in solution so that a species may be replenished as it is removed by adsorption. For example, in a buffered solution, loss of hydrogen ion by ion exchange will cause the buffer to replenish the $\mathrm{H}^{+}$. Thus, the denominator in Eq. 1 of ten will be underestimated by simply using the existing concentration of the species.

From $\mathrm{Fig} .3$, we note that $0.107 \mathrm{eq} / 1$ of $\mathrm{H}^{+}$are required to change the $\mathrm{pH}$ from 9 to 7 for our resorcinol-formaldehyde gelant. Only another $0.00223 \mathrm{eq} / 1$ of $\mathrm{H}^{+}$are needed to change the $\mathrm{pH}$ from 7 to 6 . Thus, if $n_{\text {rock }} \leq 0.106$ and if a gelant is injected at $p H=9$, we can estimate the maximum $a_{r}$ 
value associated with the rock changing the gelant $\mathrm{pH}$ to 6 . That is, $a_{r}$ is less than one $10.106 /(0.107+0.00223)]$. Similarly, if a gelant is injected at $\mathrm{pH}=7$, the maximum $\mathrm{a}_{\mathrm{r}}$ value associated with the rock changing the gelant $\mathrm{pH}$ to a value of 6 is about $48(0.106 / 0.00223)$. If the gelant contains a buffer, the $a_{r}$ values could be significantly lower since $n_{s o l}$ could be considerably greater than the values used in these examples.

Previous work ${ }^{24}$ has quantified the impact of retention $\left(a_{r}\right)$ and inaccessible pore volume $\left(a_{v}\right)$ on the degree of penetration of gelant in to a given zone in reservoirs with noncommunicating layers. For Newtonian fluids in linear flow, Eq. 4 relates the depth of penetration $\left(L_{p l}\right)$ for a species in the most-permeable layer (with properties designated with the subscript " 1 ") to the depth of penetration $\left(L_{p 2}\right)$ in a given less-permeable layer (with properties designated with the subscript " $\left.2^{n}\right)$.

$$
\begin{gathered}
\left(1+a_{r 1}-a_{v 1}\right)\left(\phi_{1} / k_{1}\right)\left[\left(F_{r}-1\right) L_{p 1}^{2} / 2+\left(\Psi_{1}+1\right) L_{p m} L_{p 1}\right]= \\
\left(1+a_{r 2}-a_{v 2}\right)\left(\phi_{2} / k_{2}\right)\left[\left(F_{r}-1\right) L_{p 2}^{2} / 2+\left(\Psi_{2}+1\right) L_{p m} L_{p 2}\right]
\end{gathered}
$$

Eq. 5 provides the analogous relation for Newtonian fluids in radial flow (with $r_{p 1}$ and $r_{p 2}$ designating radii of penetration in a given layer).

$$
\begin{aligned}
& \left(1+a_{r 1}-a_{v 1}\right)\left(\phi_{1} / k_{1}\right)\left\langle r_{p 1}^{2}\left\{F_{r} \ln \left(r_{p 1} / r_{o}\right)+\ln \left(r_{p m} / r_{p 1}\right)+\left(1-F_{r}\right) / 2+\Psi_{1} \ln \left(r_{p m} / r_{o}\right)\right]\right. \\
& \left.-r_{0}^{2}\left\{\left(\Psi_{1}+1\right) \ln \left(r_{p m} / r_{o}\right)+\left(1-F_{r}\right) / 2\right]\right)= \\
& \left(1+a_{r 2}-a_{n}\right)\left(\phi_{2} / k_{2}\right)\left(r_{p 2}^{2}\left\{F_{r} \ln \left(r_{p 2} / r_{0}\right)+\ln \left(r_{p m} / r_{p 2}\right)+\left(1-F_{r}\right) / 2+\Psi_{2} \ln \left(r_{p m} / r_{o}\right)\right]\right. \\
& \left.-r_{o}^{2}\left\{\left(\Psi_{2}+1\right) \ln \left(r_{p m} / r_{0}\right)+\left(1-F_{r}\right) / 2\right]\right\}
\end{aligned}
$$

Eqs. 6 and 7 provide the analogous relations for non-Newtonian fluids in linear flow and radial flow, respectively.

$$
\begin{aligned}
& \left(1+\mathrm{a}_{\mathrm{r} 1}-\mathrm{a}_{\mathrm{v} 1}\right)\left(\phi_{1} / \mathrm{k}_{1}\right) f\left(\mathrm{~L}_{\mathrm{p} 1}\right)=\left(1+\mathrm{a}_{\mathrm{r} 2}-\mathrm{a}_{\mathrm{v} 2}\right)\left(\phi_{2} / \mathrm{k}_{2}\right) f\left(\mathrm{~L}_{\mathrm{p} 2}\right) \\
& \left(1+\mathrm{a}_{\mathrm{r} 1}-\mathrm{a}_{\mathrm{v} 1}\right)\left(\phi_{1} / \mathrm{k}_{1}\right) f\left(\mathrm{r}_{\mathrm{p} 1}\right)=\left(1+\mathrm{a}_{\mathrm{r} 2}-\mathrm{a}_{\mathrm{v} 2}\right)\left(\phi_{2} / \mathrm{k}_{2}\right) f\left(\mathrm{r}_{\mathrm{p} 2}\right)
\end{aligned}
$$

In Eqs. 6 and 7 , the functions, $f\left(\mathrm{~L}_{\mathrm{p} 1}\right), f\left(\mathrm{~L}_{\mathrm{p} 2}\right), f\left(\mathrm{r}_{\mathrm{p} 1}\right)$, and $f\left(\mathrm{r}_{\mathrm{p} 2}\right)$, are independent of any depth of penetration other than the parameter indicated.

For propagation of $\mathrm{H}^{+*}$ or $\mathrm{OH}^{-}$fronts, the inaccessible pore volume terms $\left(\mathrm{a}_{\mathrm{v}}\right)$ can be neglected. Then, close examination of Eqs. 4 through 7 reveals that the $\left(1+a_{r}\right)$ terms will cancel if $a_{r}$ is much less than one or if $a_{r}$ values are the same in all layers. Thus, retention values must be significantly different in different layers in order to have a strong impact on the relative depth of penetration.

Now, let us examine whether pH effects can be exploited to optimize gel placement in an unfractured (radial flow) in jection well with two noncommunicating layers. A gelant will be injected without zone isolation until the gelant penetrates to a radius of $50 \mathrm{ft}$ in the most-permeable layer (layer 1). (The wellbore radius, $r_{0}$, is $0.5 \mathrm{ft}$.) We will focus on the "best-case" situation. In particular, $a_{r l}$ is assumed to be zero in the most-permeable layer. Thus, there is no retention of the gelant in layer 1 , and the $\mathrm{pH}$ front (i.e., the $\mathrm{pH}$ of the injectant) coincides with the final radius of the gelant bank $(50 \mathrm{ft})$. All of the gelant that enters this layer can form gel at the optimum $\mathrm{pH}$. Also, the resistance factor $\left(F_{r}\right)$ of the gelant is assumed to be one. This insures that the depth of penetration of gelant in the less-permeable layer (layer 2) will be minimized. 24,25 For simplicity, dispersion is neglected. ${ }^{26}$ 
In the less-permeable layer, the gelant front and the $\mathrm{pH}$ front are retarded to the extent that is determined by the factor, $a_{r 2}$. After placement of the gelant, the well is shut in to allow gelation. The gel is only allowed to form upstream of the $\mathrm{pH}$ front. Thus, gel with a residual resistance factor, $F_{T}$ is formed to a radius of $50 \mathrm{ft}$ in layer 1 . In both layers, no gel is formed downstream of the $\mathrm{pH}$ iront $\left(F_{\pi}=1\right)$.

During brine injection after gelation, we are interested in how the injection profile has changed. Figures 8 and 9 illustrate how the value for $a_{r 2}$ af fects injection profiles in a reservoir with two noncommunicating layers. This is shown as a function of permeability ratio, $k_{1} / k_{2}$, for the two layers. In the label for the $y$-axis in Figs. 8 and $9, q_{2} / q_{20}$ is the water injectivity in layer 2 a:ter the gel has formed relative to water injectivity prior to gel placement. Similarly, $q_{1} / q_{10}$ is the water injectivity in layer 1 after the gel has formed relative to water injectivity prior to gel placement. Thus, the $y$-axis represents the injectivity retained in the less-permeable layer relative to the injectivity retained in the most-permeable layer. If $\left(a_{2} / q_{20}\right) /\left(q_{1} / q_{10}\right)$ is greater than one, then the flow profile is improved by the gel treatment. In contrast, if the expression is less than one, the flow profile is impaired.

Figure 8 illustrates the case where a "weak" gel is formed (i.e, $F_{\pi}=10$ ), while Fig. 9 illustrates the case where a "strong" gel is formed (i.e, $F_{r r}=1000$ ). These figures show that even under the best circumstances, very high $a_{r 2}$ values and very high permeability ratios are required to significantly improve the injection profile. Thus, our results suggest that $\mathrm{pH}$ effects usually will not help much in eliminating the need for zone isolation during gel placement in radial flow (unfractured injection wells). A similar analysis reveals that $\mathrm{pH}$ effects are more likely to be useful during gel placement in linear flow (fractured wells). 


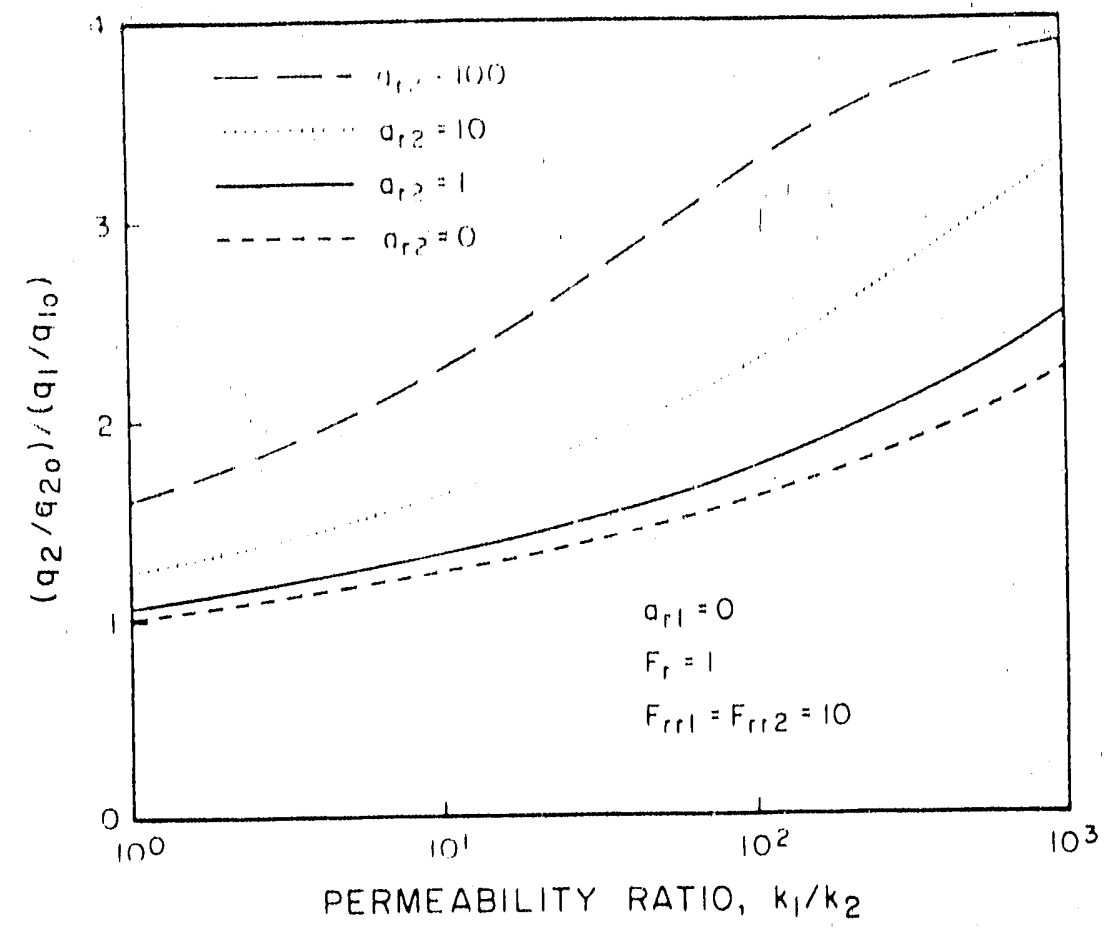

Fig. 8. Best-Case Profile Improvement in Radial Flow with a Weak Gel $\left(F_{\pi}=10\right)$.

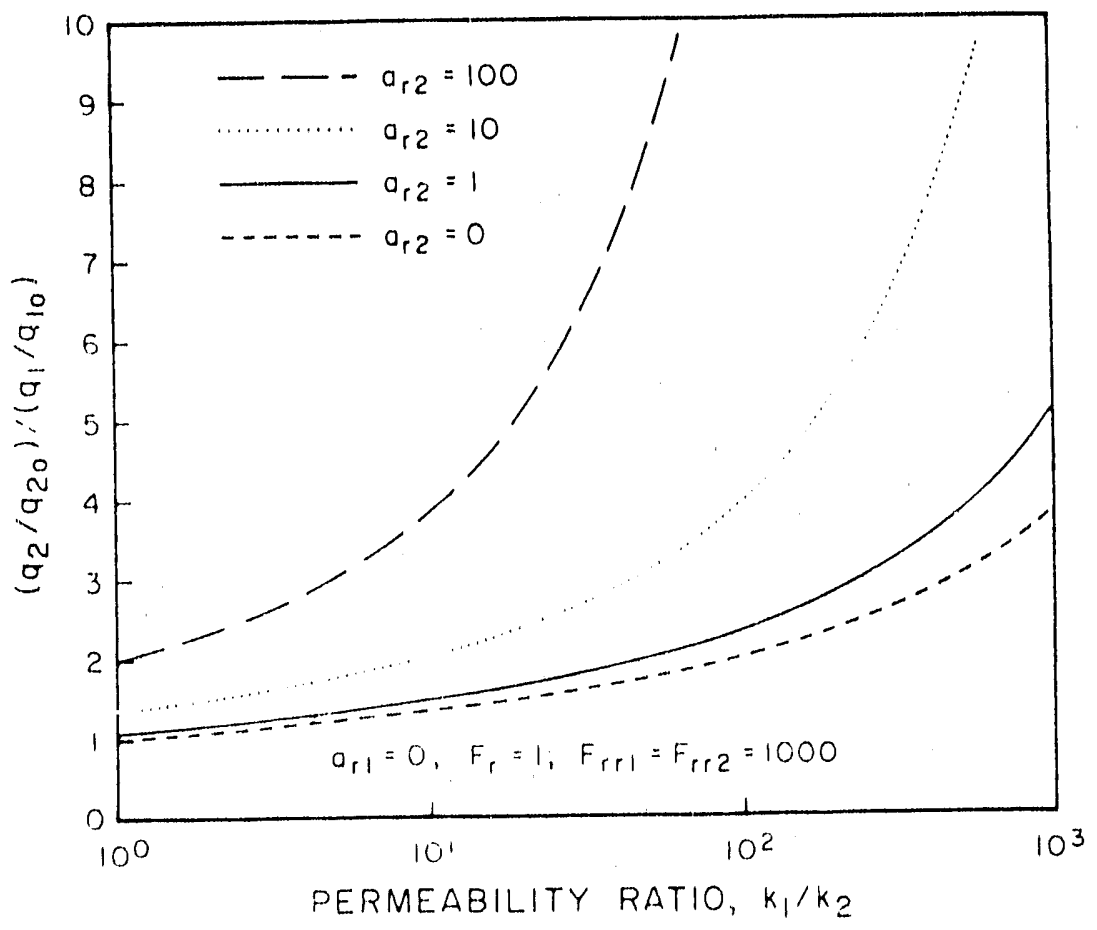

Fig. 9. Best-Case Profile Improvement in Radial Flow with a Strong Gel $\left(F_{r r}=1000\right)$. 


\section{Conclusions}

The following conclusions were reached during a study at $105^{\circ} \mathrm{F}$ of a gelant containing $3 \%$ resorcinol, $3 \%$ formaldehyde, and $0.5 \% \mathrm{KCl}$ :

1. The product formed by the reaction of resorcinol with formaldehyde depends on $\mathrm{pH}$. During studies of gelation in beakers, the strongest resorcinol-formaldehyde gels are formed at $\mathrm{pH}=9$. The inherent permeability to water for this gel (no rock) was found to be $6 \mu \mathrm{D}$.

2. As the initial pH is decreased, gel formation becomes less perfect. With an initial pH of 7 , an opaque orange-white gel is formed during beaker tests, and some free water remains after the reaction. As the initial $\mathrm{pH}$ value is decreased below 7, the final ratio of free water to $\varepsilon \mathrm{el}$ increases.

3. During core experiments, residual resistance factors are very high $\left(10^{3}-10^{4}\right)$ for gelants buffered and formed at $\mathrm{pH}=9$. Tracer studies reveal that this gel occupies $87 \%$ to $99 \%$ of the available pore volume.

4. As pH is decreased during core experiments, the gelation reaction is inhibited. In particular, as gelation $\mathrm{pH}$ decreases from 7 to 6 , residual resistance factors decrease sharply from high to low values (e.g., from $\approx 1000$ to 1 ). Tracer studies show that the fraction of the pore volume occupied by the gel generally decreases over this $\mathrm{pH}$ range.

5. In many core experiments, the results suggest that upon first exposure to a given fluid velocity, a certain amount of gel breaks down to allow a flow path through the porous medium. Flow of brine through this porous medium then appears more or less Newtonian until the previous maximum in fluid velocity is exceeded.

6. In general, residual resistance factors $\left(F_{\pi}\right)$ increased with decreased permeability. However, $F_{r r}$ values can be significantly higher in sandstones than in less-permeable carbonate cores.

7. A simple mathematical model was used to assess whether pH effects can be exploited to optimize gel placement in injection wells. Our results suggest that $\mathrm{pH}$ effects usually will not help much in eliminating the need for zone isolation during gel placement in radial flow (unf ractured injection wells). 


\section{A PRELIMINARY STUDY OF PERMEABILITY REDUCTION FOR $\mathrm{CO}_{2}$ AND WATER USING A RESORCINOL-FORMALDEHYDE GEL}

In recent years, gel treatments have been applied to reduce channeling in high-pressure gas Iloods. $3,4,46$ Laboratory work has also been performed to assess the ability of various gels to divert $\mathrm{CO}_{2}{ }^{59.30}$ With increasing interest in the use of gels to reduce channeling during high-pressure gas floods, the question arises, "How do the mechanisms for gas diversion differ from those for water diversion?" This report describes some experiments that were performed to assess how a resorcinoliormaldehyde gel will affect flow of $\mathrm{CO}_{2}$ and water in Berea sandstone.

\section{Flow of Brine and $\mathrm{CO}_{2}$ Through Berea Prior to Gel Placement}

Some initial studies were performed to characterize flow of brine and $\mathrm{CO}_{2}$ through Berea sandstone prior to g( nlacement. The Berea core that was used in this work had a total length of 14.1 $\mathrm{im}$, a cross-sectional area of $10.03 \mathrm{~cm}^{2}$, and a porosity of 0.238 . The core was cast in a metal alloy (Cerrotru ${ }^{3}$ ). The core had one internal pressure tap that was located $1.65 \mathrm{~cm}$ from the inlet sand face. The first core segment was treated as a filter, while the second core segment $(12.45-\mathrm{cm}$ length $)$ was used to measure mobilities and residual resistance factors. All experiments were performed at $105^{\circ} \mathrm{F}$ $\left(41^{\circ} \mathrm{C}\right)$. Table 8 provides a summary of many of the experimental results.

The brine contained $0.5 \% \mathrm{KCl}$ and $0.05 \mathrm{M}$ phosphate $\left(0.34 \% \mathrm{KH}_{2} \mathrm{PO}_{4}, 0.355 \% \mathrm{Na}_{2} \mathrm{HPO}_{4}\right)$. The phosphate was used to buffer the brine at $\mathrm{pH}=6.5$. (In future experiments, we will probably switch to a bicarbonate buffer to more closely imitate field brines.) The viscosity of this brine was $0.70 \mathrm{cp}$ at $105^{\circ} \mathrm{F}$. For the Eerea core, the absolute permeability to brine was $516 \mathrm{md}$. The mobility of this brine (at $105^{\circ} \mathrm{F}$ ) was $737 \mathrm{md} / \mathrm{cp}$. During injection with a 1500-psi (102-atm) back pressure, the mobility of brine was the same as that at atmospheric pressure. Figure 10 shows the tracer results that were obtained during this work. These studies involved injecting a brine bank that contained potassium iodide as a tracer. The tracer concentration in the effluent was monitored spectrophotometrically at a wavelength of $230 \mathrm{~nm}$. The circles in Fig. 10 show tracer results during brire injection before introduction of either $\mathrm{CO}_{2}$ or gel. The dispersivity value $\left(\alpha_{i}\right)$ associated with this curve is $0.060 \mathrm{~cm}$. (For all of the tracer studies described in this section, an error-function solution 52 fit the tracer curves fairly well.)

During continuous injection of $\mathrm{CO}_{2}(15.7$ to $31.4 \mathrm{ft} / \mathrm{d}$ at $1500 \mathrm{psi})$, an end-point mobility of $1466 \mathrm{md} / \mathrm{cp}$ was measured in the second core segment. During continuous brine injection $(15.7 \mathrm{ft} / \mathrm{d}$ at $1500 \mathrm{psi}$ ) after the $\mathrm{CO}_{2}$ flood, the brine mobility was found to be $628 \mathrm{md} / \mathrm{cp}$. Af ter these steps, the core was depressurized so that a second tracer study could be performed. The triangles in Fig. 10 show tracer results during brine injection with a residual $\mathrm{CO}_{2}$ saturation. The tracer curve indicates that the residual $\mathrm{CO}_{2}$ saturation occupies only $7 \%$ of the pore volume at atmospheric pressure. (This was judged using the $50 \%$ concentration level for the tracer effluent.) Also, this residual $\mathrm{CO}_{2}$ saturation did not significantly change the dispersivity $\left(\alpha_{\mathrm{f}} / \alpha_{\mathrm{i}}=1.15\right)$. 
TABLE 8

Summary of $\mathrm{CO}_{2}$ Coreflood, $105^{\circ} \mathrm{F}$

Brine contains $0.5 \% \mathrm{KCl}, 0.05 \mathrm{M}$ phosphate, $\mathrm{pH}=6.5$.

Gelant contains $3 \%$ resorcinol, $3 \%$ formaldehyde, and brine components.

Berea sandstone core: $L_{t}=14.1 \mathrm{~cm}, A=10.03 \mathrm{~cm}^{2}, k_{b}=516 \mathrm{md}, \phi=0.238$.

Mobility of brine before first $\mathrm{CO}_{2}$ and before gel: $737 \mathrm{md} / \mathrm{cp}$.

Mobility of $\mathrm{CO}_{2}$ before gel: $1466 \mathrm{md} / \mathrm{cp}$.

Mobility of brine after first $\mathrm{CO}_{2}$ but before gel: $628 \mathrm{md} / \mathrm{cp}$.

Mobility of fluids after gel placement

\begin{tabular}{|c|c|c|c|c|c|}
\hline Injectant & $\begin{array}{l}\text { Flux } \\
(f t / d)\end{array}$ & $\begin{array}{l}\text { Pore Volumes } \\
\text { Iniected }\end{array}$ & $\begin{array}{l}\text { Mobility } \\
\text { (md/co) }\end{array}$ & \multicolumn{2}{|c|}{$\begin{array}{l}\text { Residual } \\
\text { Resistance } \\
\text { Factor }\end{array}$} \\
\hline Brine & 0.202 & 1.5 & 3.40 & 185 & \\
\hline Brine & 1.57 & 0.5 & 3.18 & 197 & \\
\hline Brine & 6.28 & 2.0 & 3.25 & 193 & $\bar{F}_{r r}=192$ \\
\hline Brine & 15.7 & 2.3 & 3.27 & 192 & \\
\hline $\mathrm{CO}_{2}$ & 15.7 & 1.9 & 70.9 & 20.7 & \\
\hline $\mathrm{CO}_{2}$ & 3.14 & 1.9 & 55.0 & 26.7 & $\overline{\mathrm{F}}_{\mathrm{rr}}=23.7$ \\
\hline Brine & 15.7 & 2.2 & 25.7 & 24.4 & \\
\hline Brine & 3.14 & 1.0 & 22.7 & 27.6 & $\bar{F}_{\pi}=27.1$ \\
\hline Brine & 0.393 & 0.4 & 21.5 & 29.2 & \\
\hline
\end{tabular}

\section{Flow of Brine and $\mathrm{CO}_{2}$ Through Berea After Gel Placement}

The gelant used in this study contained $3 \%$ resorcinol and $3 \%$ formaldehyde, in addition to the components that were included with the brine. The $\mathrm{pH}$ of the gelant was 6.5 . A total of 3.6 pore volumes of gelant was in jected using a flux of $15.7 \mathrm{ft} / \mathrm{d}$ (at atmospheric pressure). The core was then shut in for three days (at atmospheric pressure) to allow gelation to occur. The gelation time was abou't five hours both for gelant that had passed through the core and for gelant that was not in jected.

Three days after shutting in the core, brine was again injected using a variety of injection rates (with 1500-psi back pressure). Residual resistance factors $\left(F_{\pi}\right)$ were determined for each injection rate. These $F_{\mathrm{rr}}$ values were determined by dividing brine mobility before gel placement $(628$ $\mathrm{md} / \mathrm{sp}$ ) by brine mobility after gel placement. Between flux values of $0.2 \mathrm{ft} / \mathrm{d}$ and $15.7 \mathrm{ft} / \mathrm{d}, \mathrm{F}_{\mathrm{m}}$ values averaged 192 during the rirst stage of brine injection after geiation (see Table 8). No visible sign of gel was found in the effluent during brine injection. 


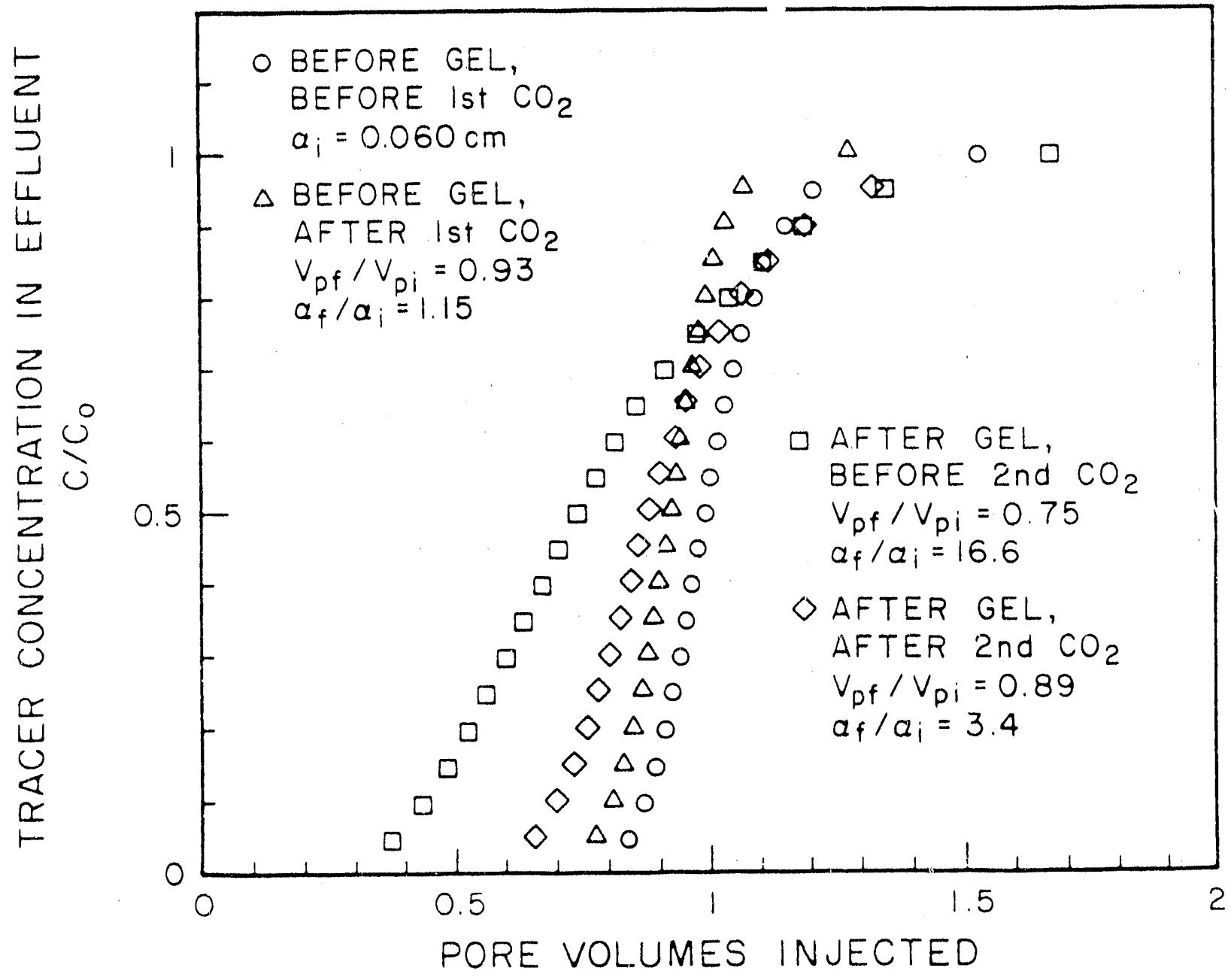

Fig. 10. Results from Tracer Studies during $\mathrm{CO}_{2}$ Experiments. 
After determination of brine $F_{\pi}$ values, the core was depressurized and additional tracer studies were conducted. The resulting tracer curve (squares in Fig. 10) indicates that $75 \%$ of the original pore volume was occupied by water. Therefore, gel and residual $\mathrm{CO}_{2}$ occupied the remaining $25 \%$ of the pore volume. The tracer curves also reveal that the dispersivity $\left(\alpha_{\mathrm{f}}\right)$ at this time was 16.6 times greater than the original dispersivity of the core $\left(\alpha_{i}\right)$.

Next, $\mathrm{CO}_{2}$ was injected at fluxes ranging from 3.14 to $15.7 \mathrm{ft} / \mathrm{d}$ (at $1500 \mathrm{psi}$ ). During this stage, the residual resistance factor for $\mathrm{CO}_{2}$ was found to average 23.7 (see Table 8). These residual resistance factors were determined by dividing $\mathrm{CO}_{2}$ mobility before gel placement $(1466 \mathrm{md} / \mathrm{cp})$ by $\mathrm{CO}_{2}$ mobility after gel placement. Then brine was injected at fluxes between $0.393 \mathrm{ft} / \mathrm{d}$ and 15.7 $\mathrm{ft} / \mathrm{d}$ (at $1500 \mathrm{psi}$ ). Residual resistance factors for water during this stage averaged 27.1. This value is seven times less than the value obtained just before the last $\mathrm{CO}_{2}$ injection.

Using a slightly lower concentration of this type of gelant, an earlier study ${ }^{30}$ found a $F_{\mathrm{rT}}$ value of 34.5 during initial brine injection after gelation. $A F_{\pi}$ value of 71.4 was found during the first $\mathrm{CO}_{2}$ injection after gelation. During a second stage of brine injection, a $F_{\mathrm{rr}}$ value of 3.0 was observed. Thus, in both this work and the earlier work, $F_{\pi}$ values for brine decreased substantially during the first brine $/ \mathrm{CO}_{2}$ cycle. Differences exist in the magnitudes of the $\mathrm{F}_{\mathrm{rr}}$ values for brine and $\mathrm{CO}_{2}$ from the two studies. A number of factors could contribute to these differences. These include different permeabilities, core lengths, and gelant $\mathrm{pH}$ values.

Finally, the core in the present study was depressurized for one last tracer study. The diamonds in Fig. 10 show these results. The curve reveals that the fraction of pore volume occupied by gel and $\mathrm{CO}_{2}$ had been reduced from $25 \%$ to $11 \%$. Also, the relative dispersivity $\left(\alpha_{\mathrm{f}} / \alpha_{\mathrm{i}}\right)$ had been reduced from 16.6 to 3.4 . These observations support the idea that the gel had broken down during the latter stages of our experiments.

We recognize that the gas will expand during depressurization prior to the tracer studies. Therefore, we are concerned about the relevance of this study to what happens in field applications. In the future, we plan to procure a high-pressure detector that will be placed in-line during our highpressure gas studies and that will allow tracer studies to be performed without depressurizing the core. The specifications of the high-pressure detector may require that our studies not exceed a pressure of 1000 psi. Ideally, future studies of this type will be conducted entirely at high pressure.

\section{Summary}

This study represents a prelininary effort to examine how gels reduce permeability to highpressure gas relative to that for water. The procedures that were examined during this study will provide a basis for future experiments. 


\section{A PRELIMINARY STUDY OF PERMEABILITY REDUCTION FOR OIL AND WATER USING A RESORCINOL-FORMALDEHYDE GEL}

Several researchers ${ }^{56-60}$ reported that some polymers and gels can reduce permeability to water more than to oil. The objectives of our research in this area are to examine the reason why the disproportionate permeability reduction occurs and to identify conditions that maximize this phenomenon. We are also examining whether hysteresis of oil-water relative permeability curves occurs during the "pump-in, pump-out" sequence used during gel treatments in production wells. This report describes our initial work with a refined oil in Berea sandstone. This fluid-rock system is strongly water-wet. In future work, we will examine other gels, other lithologies, and fluid-rock systems of intermediate wettability.

\section{Effect of Flow-Direction Reversal on End-Point Permeabilities Prior to Gel Placement}

The core used in this work had a total length of $13.57 \mathrm{~cm}$, and a cross-sectional area of 10.03 $\mathrm{cm}^{2}$. The core was cast in a metal alloy and had one internal pressure tap located at $2.13 \mathrm{~cm}$ from the inlet rock face. The core was not fired. All experiments were performed at $105^{\circ} \mathrm{F}$.

Some initial studies were performed to evaluate the effect of flow-direction reversal on end-point oil and water permeabilities. The brine contained $0.5 \% \mathrm{KCl}$. A Berea sandstone core (635-md absolute permeability to brine) was used as the porous medium, and a refined oil (Soltrol-130॰) was used as the oil phase. The rock and fluid properties involved are listed in Table 9. The core was first saturated with brine, and the porosity and the permeability to brine were determined. It then went through a cycle of oilflooding followed by waterflooding to establish irreducible oil saturation. The end-point oil and water permeabilities were determined at the irreducible water saturation af ter the oilflood and the irreducible oil saturation af ter the waterflood, respectively. Then the flow direction was reversed and the procedure described above was repeated so that the effect of hysteresis could be determined. In order to verify that the results were reproducible, each step in the procedure described above was repeated twice. Table 10 provides a summary of the experimental results. Table 10 shows that the results were reproducible, and the end-point oil permeability was not affected by the flow-direction reversal. The end-point water permeability was slightly lower when the flow-direction was reversed. However, the effect was not large enough to be significant.

TABLE 9

Rock and Fluid Properties for Oil/Water Experiments

Core Properties:

Core Type: Berea Sandstone

$$
\begin{array}{ll}
L_{t}=13.57 \mathrm{~cm} & V_{p}=33.5 \mathrm{mid} \\
r=1.8 \mathrm{~cm} & \phi=0.244 \\
A=10.12 \mathrm{~cm}^{2} & k_{b}=635 \mathrm{md}
\end{array}
$$

Fluid Properties:

Brine: $0.5 \% \mathrm{KCl}, \mu_{\mathrm{b}}=0.6 \mathrm{cp}\left(105^{\circ} \mathrm{F}\right)$

Oil: Soltrol $-130, \mu_{0}=1.05 \mathrm{cp}\left(105^{\circ} \mathrm{F}\right)$ 
TABLE 10

Effect of Flow-Direction Reversal on End-Point

Permeabilities Prior to Gel Treatment

Drainage - 1

Drainage - 2

Drainage - 3 (reversed)

Drainage - 4 (reversed)

Imbibition - 1

Imbibition - 2

Imbibition - 3 (reversed)

Imbibition - 4 (reversed)

$\frac{S_{w r}}{0.13} \quad \frac{k_{0}^{\prime}(m d)}{624} \quad \frac{K_{0}^{\prime}(n . d)}{624.5}$

625

$0.23 \quad 570$

$0.15 \quad 654$

$\underline{S_{o r}} \quad \underline{k_{w}^{\prime}(m d)} \quad \underline{k_{w}^{\prime}(m d)}$

$0.36 \quad 89$

$0.32 \quad 78$

$0.37 \quad 69$

$0.40 \quad 63$
83.5

66.0

Tracer studies were performed during this process. These studies involved injecting a brine bank that contained potassium iodide as a tracer. The tracer concentration in the effluent was monitored spectrophotometrically at a wavelength of $230 \mathrm{~nm}$. The tracer breakthrough curves from our tracer studies are shown in Fig. 11. The results of tracer studies are summarized in Table 11. In Table $11, \mathrm{~V}_{\mathrm{p} 50 \%}$ refers to the number of pore volumes of tracer injected at the time when the $50 \%$ level of tracer concentration was observed in the effluent. No significant changes in pore volume and dispersivity were observed as a result of the flow-direction reversal.

Figure 12 presents the tracer breakthrough curves with and without residual oil present. As shown in the plot, an error-function solution fits the tracer curve fairly well when no residual oil was present. Howe' ir, with the residual oil present, the error-function solution no longer fits the tracer curve well. There was a delay in reaching the injection tracer concentration compared with the error-function solution. The volume of tracer injected when the tracer concentration reached $50 \%$ of its injection value was also significantly less than the pore volume calculated by the material balance. (In Fig. 12 the $V_{p 50 \%}$ value for the case with $S_{o r}=0.36$ was shifted from 0.4 to 1 to allow an easier comparison with the error-function solution.)

TABLE 11

Summary of the Results of Tracer Studies During Oil/Water Experiments

$100 \%$ Brine Sarurated

Imbibition - 1

Imbibition - 2

Imbibition - 3 (Reversed)

Imbibition - 4 (Reversed)

After Gel Treatment (Reversed)
$\underline{V_{\mathrm{D} 50 \%}} \quad \underline{\alpha(\mathrm{cm})}$

i.0 $\quad 0.06$

$0.407 \quad 2.27$

$0.418 \quad 2.20$

$0.418 \quad 2.20$

$0.428 \quad 1.90$

$0.446-1.86$ 


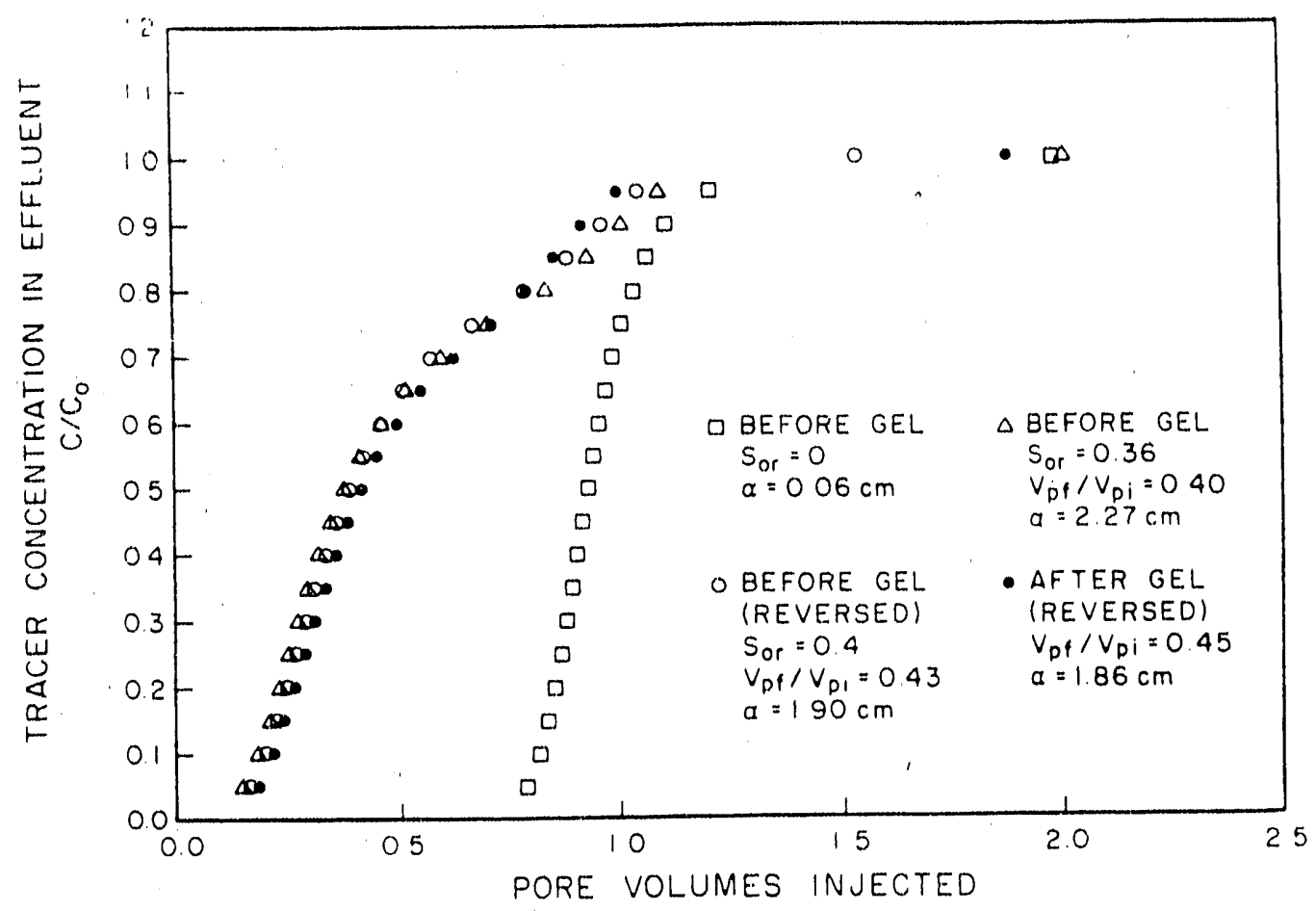

Fig. 11. Tracer Breakthrough Curves from Tracer Studics During Oil/Water Experiments.

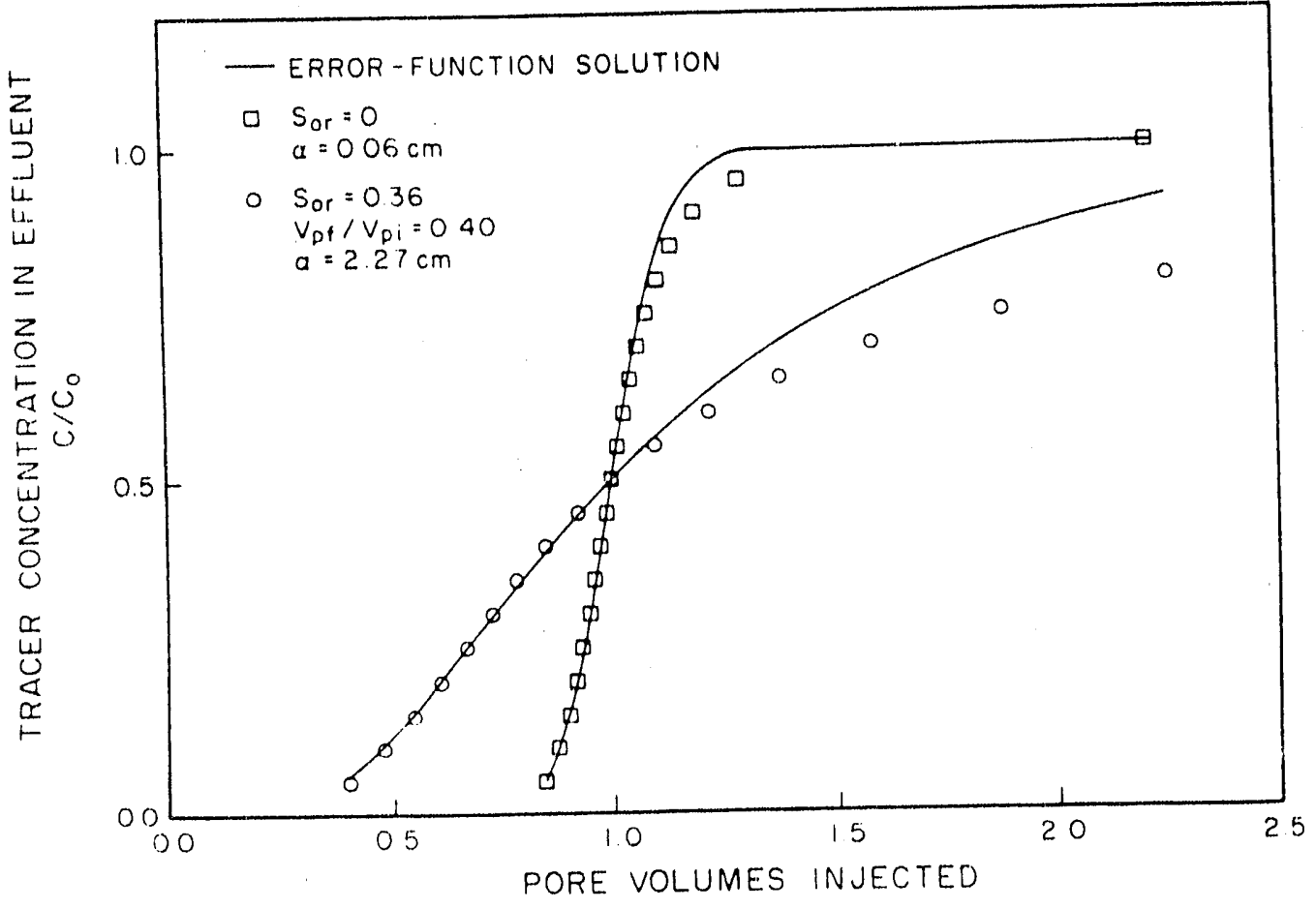

Fig. 12. Tracer Breakthrough Curves with/without Residual Oil Present. 


\section{Permeability Reduction After Gel Treatment}

In order to simulate the "pump-in, pump-out" sequence used during gel treatments in production wells, the gelant was injected into the core from one direction and residual resistance factors $\left(F_{r r}\right)$ were measured in the opposite direction.

Resistance factors $\left(F_{r}\right)$ and effluent $\mathrm{pH}$ were monitored during the entire gelant-injection process. $F_{r}$ values were near one during gelant injection. Effluent samples were collected and monitored to determine whether the gelation characteristics of the effluent differed from those of gelant that had not been injected. After injection of the gelant, the ccre was shut in for five days (at $105^{\circ} \mathrm{F}$ ). After shut-in, brine was injected from the opposite direction to determine the residual resistance factors $\left(F_{r r}\right)$ af ter gel treatment.

The gelant used in this study contained $3 \%$ resorcinol and $3 \%$ formaldehyde, in addition to the components that were included with the brine. Retention studies performed in cores that contained a residual oil saturation revealed that no significant level of resorcinol partitioned into the oil phase. We also examined how' the presence of oil affects gelation. The presence of the refined oil had no effect on the gelation time or the appearance of the gel. No significant solubility of either resorcinol or formaldehyde was found in Soltrol-130\%.

Acetic acid $(0.05 \mathrm{M})$ was used as a buffer, and the $\mathrm{pH}$ of the gelant was adjusted to 5 prior to injection. A total of 3.7 pore volumes of gelant was injected using a flux of $15.6 \mathrm{ft} / \mathrm{d}$. The core was then shut in for five days to allow gelation to occur. After the shut-in period, brine was injected from the opposite direction and the residual resistance factor $\left(F_{\pi}\right)$ was found to be very close to 1 . A comparison of the results of the tracer tests before and after gel treatment is shown in Table 11. This table shows no significant changes in either pore volume or dispersivity as a result of gel treatment. We suspect that larger $F_{n \pi}$ values would have been observed if gelation had been allowed to occur at higher $\mathrm{pH}$ values. This will be investigated in future studies.

\section{Summary}

This study represents a preliminary effort to examine how gels reduce permeabilities to oil and water. The study also examines the effect of flow-direction reversal on oil and water permeabilities. The procedures that were examined during this study will provide a basis for future experiments. 


\section{RHEOLOGY OF CHROMIUM(III)-XANTHAN GELS AND GELANTS IN POROUS MEDIA}

For many years, researchers have been aware of the non-Newtonian rheology of polymer solutions in porous media. ${ }^{61.64}$ This has prompted some to speculate that non-Newtonian rheology may be exploitable in eliminating the need for zone isolation during gel placement. ${ }^{65}$ We have examined the potential of this idea by performing calculations using eight different models of nonNewtonian rheology ${ }^{25}$ (including shear-thickening models, shear-thinning models, and various combinations). These calculations suggest that the rheology of existing polymers will not eliminate the need for zone isolation during gel placement in unf ractured injection wells. However, the validity of these calculations rests on several assumptions that need to be examined experimentally. First, the calculations assume that prior to gelation, the rheology of polymer solutions with crosslinkers is the same as that of polymer solutions without crosslinkers. Second, af ter gelation, the residual resistance factors are assumed to be independent of velocity and permeability. Third, the calculations assume that the wells are shut-in at the time gelation occurs. The calculations also wsume that gels are not mobile. This report describes results of experiments that were designed to test some of the above assumptions for chromium(III)-xanthan gels in porous media.

\section{Gelant Rheology in Porous Media Prior to Gelation}

Our first experiments were designed to test whether the rheology of xanthan solutions in porous media prior to gelation depends on the presence of chromium. These experiments were performed using aqueous solutions that contained $3000-\mathrm{ppm}$ xanthan, $3 \% \mathrm{NaCl}$, and $0.3 \% \mathrm{CaCl}_{2}$ at $85^{\circ} \mathrm{F}\left(29^{\circ} \mathrm{C}\right)$. When chromium(III) was included, a concentration of $90-\mathrm{ppm}$ was used. Pfizer provided both the xanthan (Flocon 4800\%) and the chromium (X-link 2000॰) that were used in this work. In these core experiments, Berea sandstone cores were used that had diameters of $1.27 \mathrm{~cm}$ and lengths of $15.24 \mathrm{~cm}$. All cores had one internal pressure tap that was located $2.54 \mathrm{~cm}$ from the inlet sand face. The cores had not been fired.

Viscosity vs. shear-rate data are shown in Fig. 13 for five 3000 -ppm xanthan solutions:

(a) without chromium, befo-e injection;

(b) without chromium, effluent from a 293-md core (after injecting 10 pore volumes);

(c) with 90-ppm $\mathrm{Cr}^{3+}$ (freshly mixed), before injection;

(d) with $90-\mathrm{ppm} \mathrm{Cr}{ }^{3+}$, effluent from a $293-\mathrm{md}$ core (after injecting 10 pore volumes, 0.7 hours af ter gelant preparation);

(e) with 90-ppm $\mathrm{Cr}^{3+}$, effluent from a 293 -md core (after injecting 53 pore volumes, 4.5 hours after gelant preparation).

Except possibly at very low shear rates, there is no significant difference in rheology between the five different solutions-at least, not in the first several hours of the gelation process. The gelation time (as measured by monitoring viscosity at $1.75 \mathrm{~s}^{-1}$ ) was 10.5 hours for gelant that was not in jected into a core. The gelation time was about 8 hours for effluent that was collected after injection at a flux (volumetric flow rate per unit area) of $225 \mathrm{ft} / \mathrm{d}$. Furthermore, ine gel formed from this efflueric was noticeably more rigid than the gel that was formed from gelant that had not been injected into the core.

The effect of $\mathrm{Cr}^{3+}$ on xanthan rheology (during the first seven hours of the gelation process) in 293-md Berea sandstone is shown in Fig. 14. Resistance factors shown in Fig. 14 are also listed in Tables 12 and 13. These tables list data in the chronological order in which they were collected. For the core experiments, the rheology of xanthan solutions without $\mathrm{Cr}^{3+}$ were determined first (Table 12a). Then, brine was injected to displace the xanthan and to determine residual resistance 


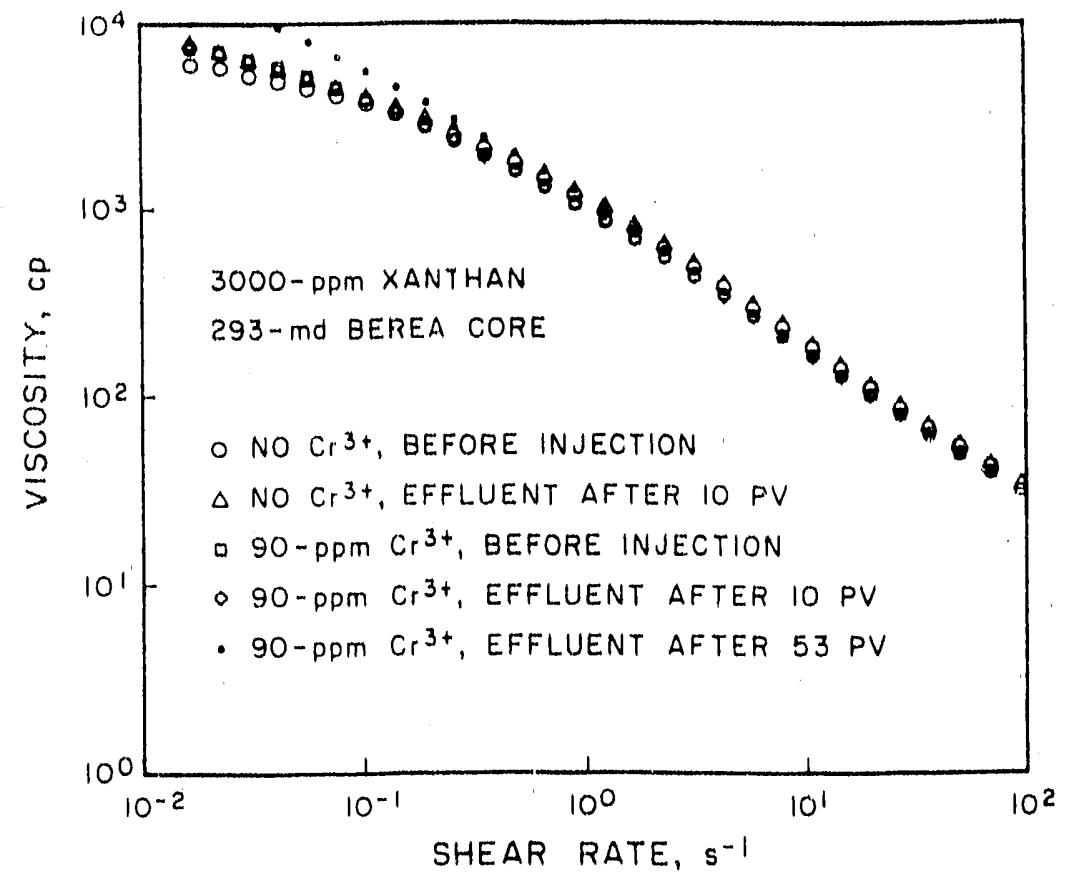

Fig. 13. Effect of $\mathrm{Cr}^{3+}$ and Flow through 293-md Berea on the Viscosity of 3000-ppm Xanthan.

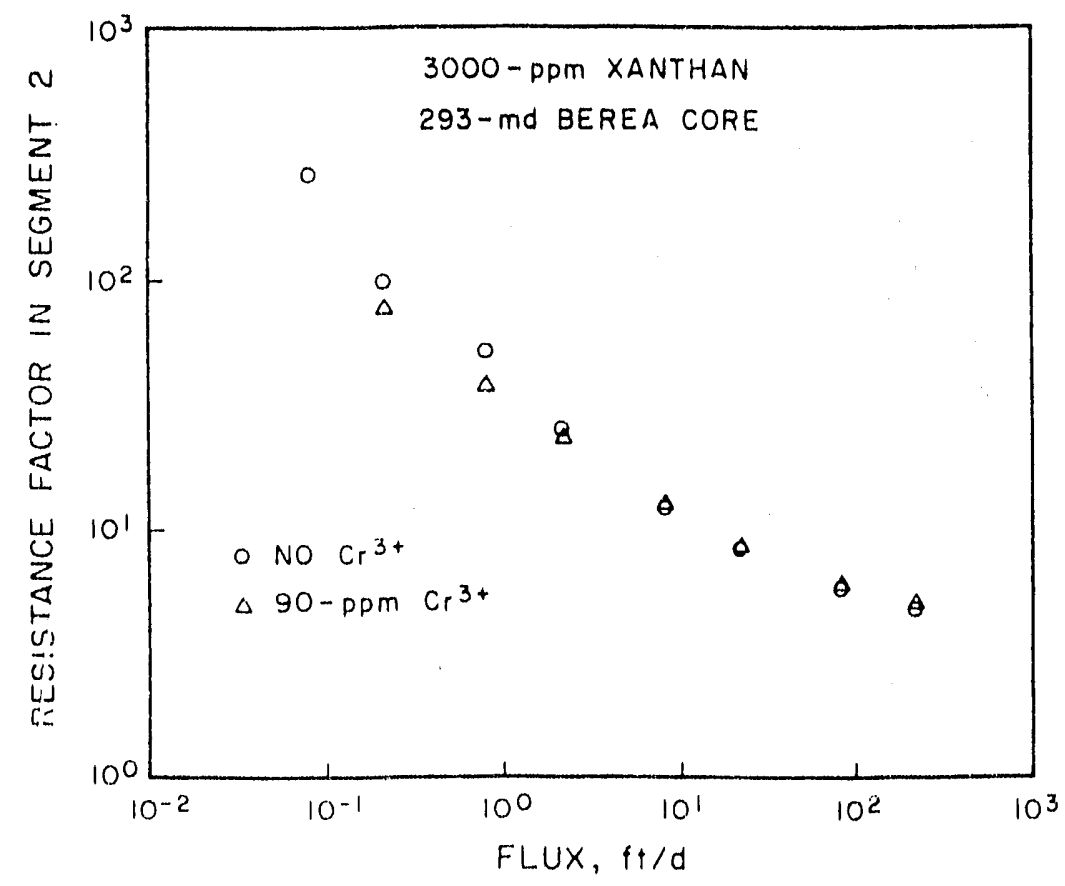

Fig. 14. Effect of $\mathrm{Cr}^{3+}$ on Rheology of 3000-ppm Xanthan in 293-md Berea. 
factors as a function of flow rate (Table 12b). Next, xanthan solutions with 90-ppm $\mathrm{Cr}^{3+}$ were injected to determine resistance factors as a function of flow rate (Table 13a). This step was performed as early in the gelation process as was practical. Then, the core was shut-in for several days to allow gelation to proceed. Gel and gelant were removed from the flow lines, and gel was scraped from the sand faces. Finally, brine was injected to determine residual resistance factors as a function of flow rate (Table $13 \mathrm{~b}$ ). Resistance factors and residual resistance factors were monitored in both core segments.

TABLE 12

Rheology of 3000-ppm Xanthan (without $\mathrm{Cr}^{3+}$ ) in 293-md Berea Sandstone

Total core length $=15.24 \mathrm{~cm}$, diameter $=1.27 \mathrm{~cm}, \phi=0.21$.

Segment 1 length $=2.54 \mathrm{~cm}$, segment 2 length $=12.7 \mathrm{~cm}, 85^{\circ} \mathrm{F}$.

(d) Resistance factors during injection of $3000-\mathrm{ppm}$ xanthan, $3 \% \mathrm{NaCl}, 0.3 \% \mathrm{CaCl}_{2}$.

\begin{tabular}{|c|c|c|c|}
\hline \multirow{2}{*}{$\begin{array}{l}\text { Flux } \\
\text { (ft/d) }\end{array}$} & \multirow{2}{*}{$\begin{array}{l}\text { Injected } \\
\text { Pore Volumes }\end{array}$} & \multicolumn{2}{|c|}{ Resistance Factors } \\
\hline & & Segment 1 & Segment 2 \\
\hline 225 & 17.2 & 4.1 & 4.8 \\
\hline 84.4 & 14.0 & 5.5 & 5.8 \\
\hline 22.5 & 4.1 & 8.7 & 8.4 \\
\hline 8.44 & 1.6 & 14.4 & 12.4 \\
\hline 2.25 & 0.4 & 31.7 & 26.0 \\
\hline 0.844 & 0.3 & 67.7 & 52.7 \\
\hline 0.225 & 1.6 & $106-189^{\circ}$ & 101 \\
\hline 0.0844 & 1.6 & $300-900^{\circ}$ & 268 \\
\hline 0.225 & 1.7 & 323 & 125 \\
\hline 0.844 & 0.3 & 121 & 47.9 \\
\hline 2.25 & 1.2 & 58.0 & 26.2 \\
\hline 8.44 & 4.7 & 22.3 & 12.6 \\
\hline 22.5 & 6.7 & 12.4 & 8.2 \\
\hline 84.4 & 6.5 & 6.5 & 5.7 \\
\hline 225 & 9.3 & 4.6 & 4.6 \\
\hline & 71.2 & & \\
\hline
\end{tabular}

* Resistance factors in this segment rose steadily during the course of injection at this rate.

(b) Residual resistance factors during injection of brine with $3 \% \mathrm{NaCl}, 0.3 \% \mathrm{CaCl}_{2}$.

\begin{tabular}{|c|c|c|c|}
\hline Flux & Injected & \multicolumn{2}{|c|}{ Residual Resistance Factors } \\
\hline$(\mathrm{ft} / \mathrm{d})$ & Pore Volumes & Segment 1 & Segment 2 \\
\hline 225 & 110.2 & 1.1 & 1.0 \\
\hline 84.4 & 7.9 & 1.2 & 1.0 \\
\hline 22.5 & 7.9 & 1.9 & 1.1 \\
\hline 8.44 & 5.0 & 2.6 & 1.3 \\
\hline 2.25 & 0.8 & 4.0 & 1.4 \\
\hline & $\overline{131.8}$ & & \\
\hline
\end{tabular}


TABLE 13

Rheology of 3000-ppm Xanthan with 90-ppm $\mathrm{Cr}^{3+}$ in 293-md Berea Sandstone

Total core length $=15.24 \mathrm{~cm}$, diamster $=1.27 \mathrm{~cm}, \phi=0.21$.

Segment 1 length $=2.54 \mathrm{~cm}$, segment 2 length $\left.=12.7 \mathrm{~cm}, 85^{\circ}\right)^{\circ}$.

(a) Resistance factors during injection of $3000-p p m$ xanthan, $90-p p m \mathrm{Cr}^{3+}, 3 \% \mathrm{NaCl}, 0: 3 \% \mathrm{CaCl}_{2}$.

\begin{tabular}{|c|c|}
\hline $\begin{array}{l}\text { Flux } \\
(\mathrm{ft} / \mathrm{d})\end{array}$ & $\begin{array}{c}\text { Injected } \\
\text { Pore Volumes }\end{array}$ \\
\hline 225 & 27.3 \\
\hline 84.4 & 18.9 \\
\hline 22.5 & 5.2 \\
\hline 8.44 & 1.4 \\
\hline 2.25 & 1.2 \\
\hline 0.844 & 0.4 \\
\hline \multirow[t]{2}{*}{0.225} & 0.1 \\
\hline & 54.5 \\
\hline
\end{tabular}

\begin{tabular}{|c|c|c|}
\hline \multicolumn{2}{|c|}{ Resistance Factors } & Time since gelant \\
\hline Segment 1 & Segment 2 & formulation (hrs) \\
\hline $5.5-6.1$ & 4.9 & 1.0 \\
\hline $8.2-11.1^{\circ}$ & 5.9 & 1.6 \\
\hline $15.5-25.7^{\circ}$ & 8.4 & 2.3 \\
\hline $49-67^{\circ}$ & 12.5 & 2.8 \\
\hline $164-783^{\circ}$ & 23.0 & 4.6 \\
\hline $1970-3870^{\circ}$ & 37.3 & 5.9 \\
\hline $14600-16300^{\circ}$ & 76 & 6.9 \\
\hline
\end{tabular}

* Resistance factors in this segment rose steadily during the course of injection at this rate.

(b) Residual resistance factors during injection of brine with $3 \% \mathrm{NaCl}, 0.3 \% \mathrm{CaCl}_{2}$. Core had been shut-in for four days to allow gelation.

Gel was removed from the sand faces prior to brine injection.

Viscosity of first pore volume of effluent during brine injection was $23.5 \mathrm{cp} @ 11 \mathrm{~s}^{-1}$.

\begin{tabular}{|c|c|c|c|}
\hline Flur & Injected & \multicolumn{2}{|c|}{ Residual Resistance Factors } \\
\hline$(\mathrm{f} t / d)$ & Pore Volumes & Segment 1 & Segment 2 \\
\hline 0.225 & 2.0 & $20-1100^{\circ}$ & $8.0^{\circ}$ \\
\hline 0.844 & 0.8 & 745 & 3.4 \\
\hline 2.25 & 1.2 & 431 & 2.3 \\
\hline 8.44 & 2.2 & 130 & 1.7 \\
\hline 22.5 & 3.4 & 70 & 1.4 \\
\hline 84.4 & 7.4 & 14 & 1.1 \\
\hline 225 & 30.0 & 12 & 1.1 \\
\hline 84.4 & 13.9 & 9.6 & 1.2 \\
\hline 22.5 & 4.5 & 9.0 & 1.3 \\
\hline 8.44 & 3.6 & 10.8 & 1.6 \\
\hline 2.25 & 1.4 & 19.3 & 2.0 \\
\hline 0.844 & 0.5 & 52 & 3.1 \\
\hline & 70.9 & & \\
\hline
\end{tabular}

* Residual resistance factors in this segment rose steadily during the course of injection at this rate.

* Value at the end of 2 pore volumes. Unsteady values observed prior to this point. 
Figure 14 shows that, during the first seven hours of the gelation process, the presence of 90 $\mathrm{ppm} \mathrm{Cr}{ }^{3+}$ does not significantly af fect the rheology in porous media of a 3000 -ppm xanthan solution. The same conclusion is reached for studies in 164-ma Berea sandstone (see Figs, 15 and 16, and Tables 14 and 15 ). This conclusion also appears valid when a residual oil saturation is present (see Fig. 17 and Tables 16 and 17 ).

Some plugging (i.e., progressive increase in apparent resistance factor) of the first core segment was noted during the experiments with 293-md Berea (see Tables 12a, 13a, and 13b). However, this plugging was observed to a lesser extent during the experiments with $164-\mathrm{md}$ Berea (see Table 15). The plugging appeared to be most severe at low flow rates. Sometimes, exposure to high flow rates could reverse the plugging to some extent (see Table 13b). Plugging was not observed in the core with a residual oil saturation. We believe that this plugging is an experimental artifact during our experiments. Based on our data, its presence or absence should not be correlated with permeability or oil saturation.

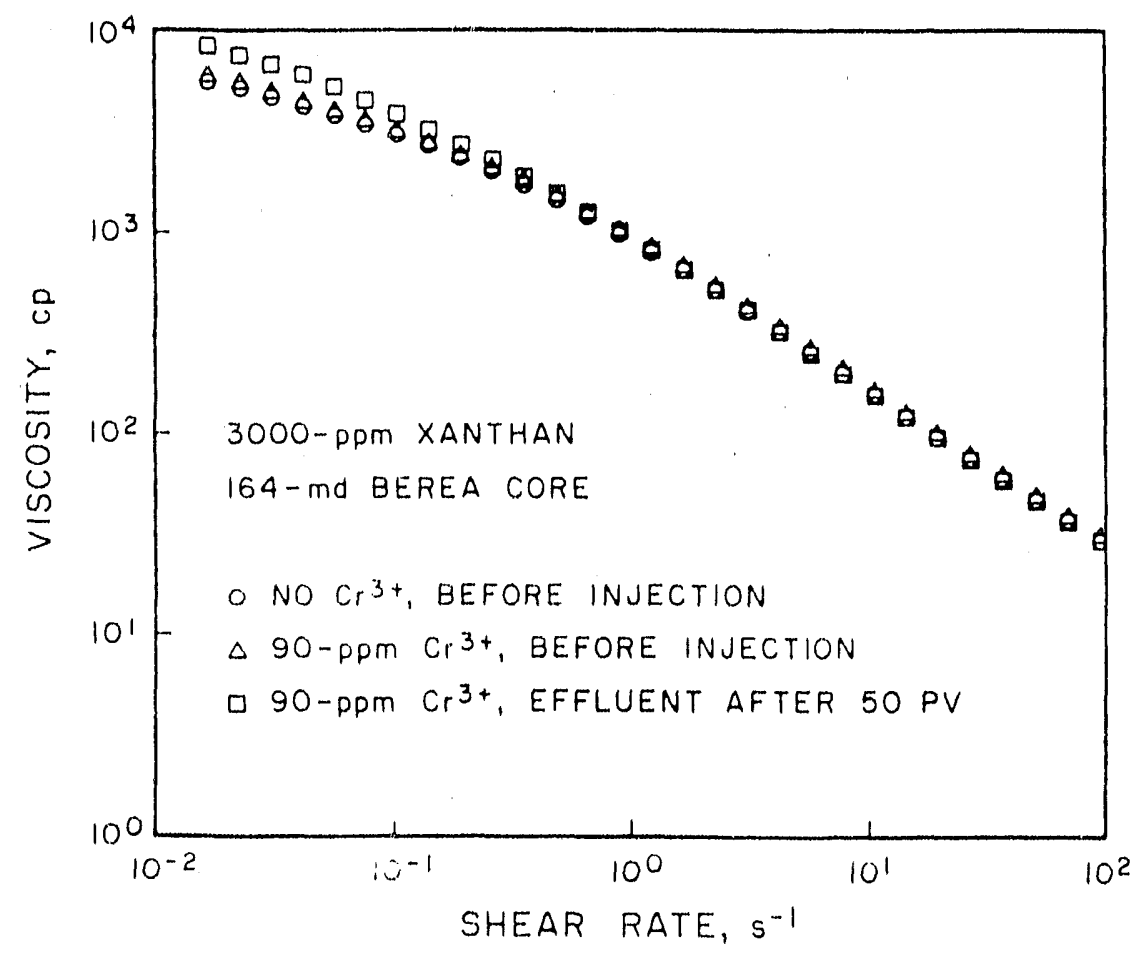

Fig. 15. Effect of $\mathrm{Cr}^{3+}$ and Flow through 164-mi Berea on the Viscosity of 3000-ppm Xanthan. 


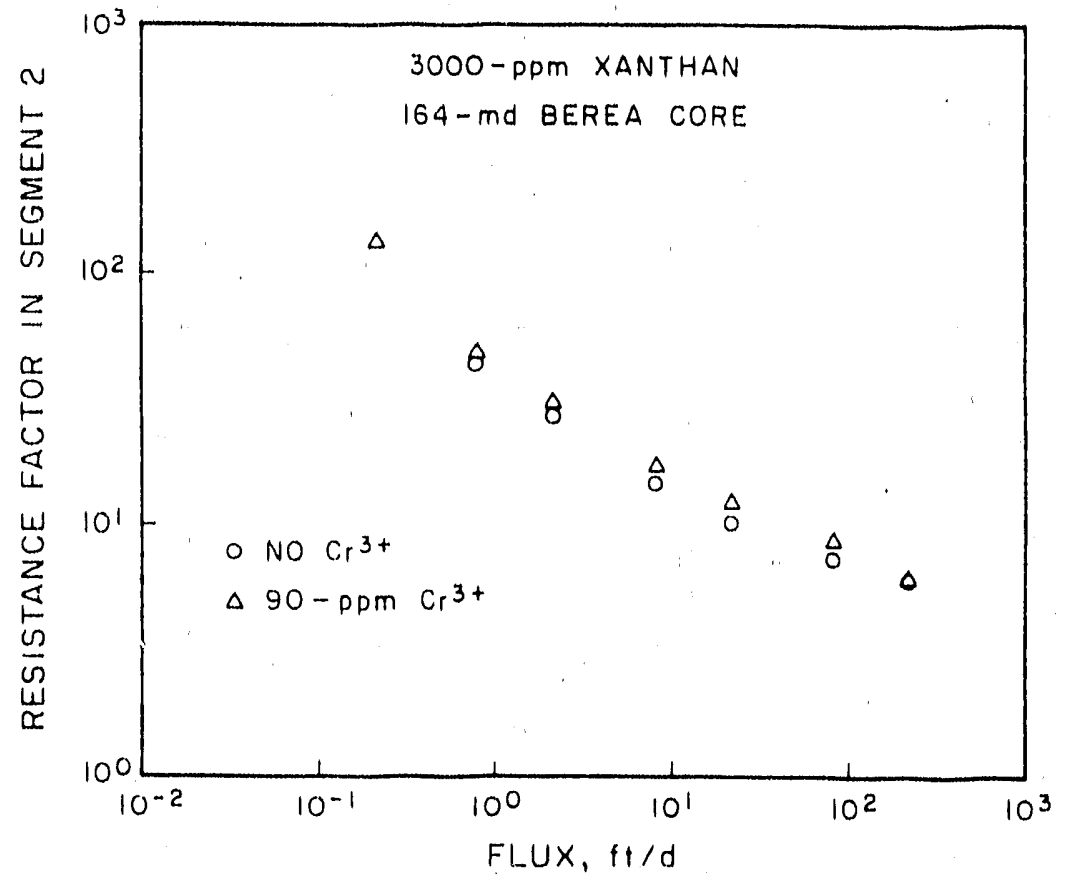

Fig. 16. Effect of $\mathrm{Cr}^{3+}$ on Rheology of 3000-ppm Xanthan in 164-md Berea.

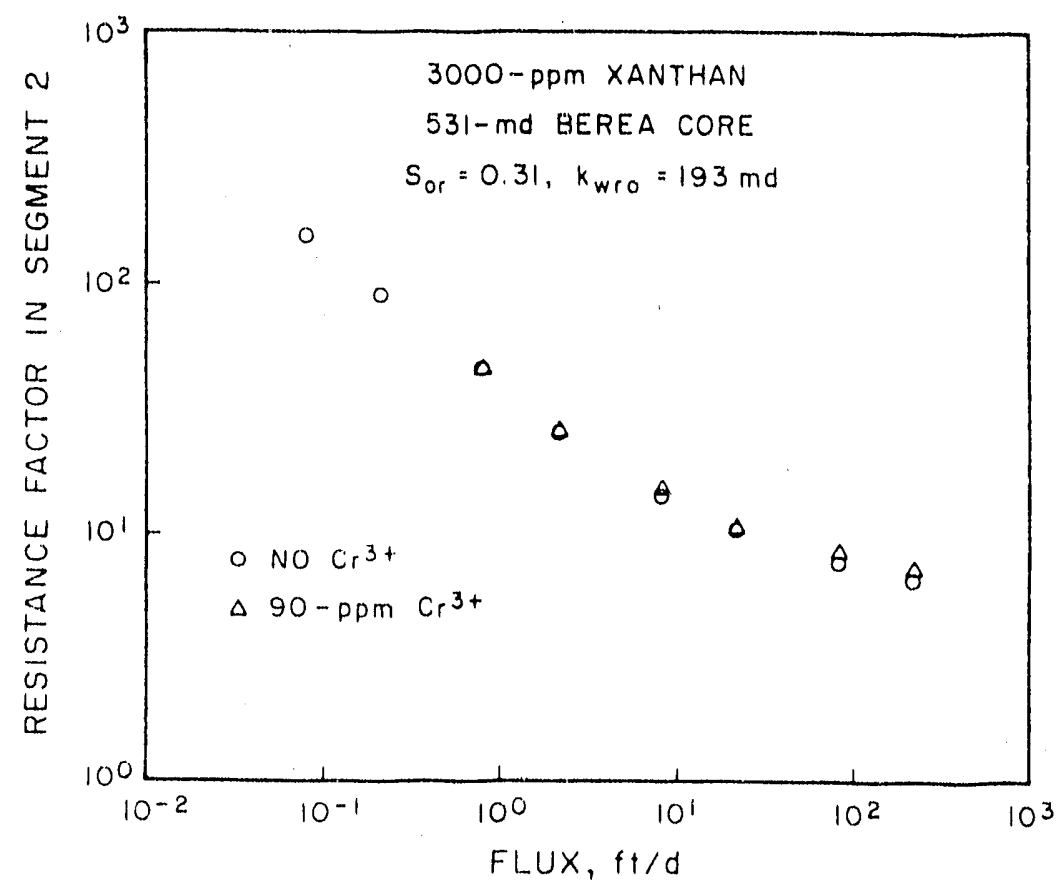

Fig. 17. Effect of $\mathrm{Cr}^{3+}$ on Rheology of $3000-\mathrm{ppm}$ Xanthan in 531 -md Berea with $\mathrm{S}_{\mathrm{or}}=0.31$. 
TABLE 14

Rheology of 3000-ppm Xanthan (without $\mathrm{Cr}^{3+}$ ) in 164-md Berea Sandstone

Total core length $=15.24 \mathrm{~cm}$, diameter $=1.27 \mathrm{~cm}, \phi=0.19$. Segment 1 length $=2.54 \mathrm{~cm}$, segment 2 length $=12.7 \mathrm{~cm}, 85^{\circ} \mathrm{F}$.

(a) Resistance factors during injection of $3000-\mathrm{ppm}$ xanthan, $3 \% \mathrm{NaCl}, 0.3 \% \mathrm{CaCl}_{2}$.

\begin{tabular}{|c|c|}
\hline $\begin{array}{l}\text { Flux } \\
\text { (ft/d) }\end{array}$ & $\begin{array}{l}\text { Injected } \\
\text { Pore Volumes }\end{array}$ \\
\hline 22 & 60.0 \\
\hline 83.4 & 16.0 \\
\hline 22.2 & 4.1 \\
\hline 8.34 & 3.3 \\
\hline 2.22 & 0.9 \\
\hline 0.834 & 5.6 \\
\hline 2.22 & 0.7 \\
\hline 8.34 & 2.1 \\
\hline 22.2 & 5.9 \\
\hline 83.4 & 9.5 \\
\hline 222 & 13.0 \\
\hline & 121.1 \\
\hline
\end{tabular}

\begin{tabular}{|c|c|}
\hline \multicolumn{2}{|c|}{ Resistance Factors } \\
\hline Segment 1 & Segment 2 \\
\hline 6.0 & 6.1 \\
\hline 7.3 & 7.4 \\
\hline 10.7 & 10.3 \\
\hline 15.8 & 14.8 \\
\hline 29.1 & 2.7 .2 \\
\hline 48.0 & 43.8 \\
\hline 29.8 & 26.7 \\
\hline 15.8 & 14.5 \\
\hline 10.5 & 10.3 \\
\hline 7.3 & 7.4 \\
\hline 6.0 & 6.2 \\
\hline
\end{tabular}

(b) Residual resistance factors during injection of brine with $3 \% \mathrm{NaCl}, 0.3 \% \mathrm{CaCl}_{2}$.

$\begin{array}{cc}\begin{array}{c}\text { Flux } \\ \text { (ft/द) }\end{array} & \begin{array}{c}\text { Injected } \\ \text { Pore Volumes }\end{array} \\ 222 & 100.0 \\ 83.4 & 24.5 \\ 22.2 & 2.8 \\ 8.34 & 2.3 \\ 2.22 & 1.1 \\ 0.834 & \text { Total: } \frac{0.3}{131.0}\end{array}$

Residual Resistance Factors

Segment 1 Segment 2

$\begin{array}{rr}1.8 & 1.7 \\ 2.0 & 1.8 \\ 2.4 & 2.2 \\ 3.2 & 2.6 \\ 5.3 & 4.0 \\ 10.4 & 6.2\end{array}$


TABLE 15

Rheology of 3000-ppm Xanthan with 90-ppm $\mathrm{Cr}^{3+}$ in 164-md Berea Sandstone

Total core length=15.24 cm, diameter $=1.27 \mathrm{~cm}, \phi=0.21$.

Segment 1 length $=2.54 \mathrm{~cm}$, segment 2 length $=12.7 \mathrm{~cm}, 85^{\circ} \mathrm{F}$.

(a) Resistance factors during injection of $3000-\mathrm{ppm}$ xanthan, $90-\mathrm{ppm} \mathrm{Cr}^{3+}, 3 \% \mathrm{NaCl}, 0.3 \% \mathrm{CaCl}_{2}$.

\begin{tabular}{|c|c|c|c|c|}
\hline Flux & Injected & \multicolumn{2}{|c|}{ Resistance Factors } & \multirow{2}{*}{$\begin{array}{l}\text { Time since gelant } \\
\text { formulation (hrs) }\end{array}$} \\
\hline$(\mathrm{ft} / \mathrm{d})$ & Pore Volumes & Segment 1 & Segment 2 & \\
\hline 222 & 8.9 & 6.5 & 6.0 & 0.5 \\
\hline 83.4 & 42.5 & 9.9 & 8.5 & 2.0 \\
\hline 22.2 & 4.1 & 14.9 & 12.0 & 2.6 \\
\hline 8.34 & 2.6 & 21.4 & 16.8 & 3.6 \\
\hline 2.22 & 1.1 & 36.4 & 29.8 & 5.4 \\
\hline 0.834 & 0.6 & 61.6 & 47.5 & 7.3 \\
\hline 0.222 & 0.1 & 181 & 132 & 8.3 \\
\hline & 59.9 & & & \\
\hline
\end{tabular}

(b) Residual resistance factors during injection of brine with $3 \% \mathrm{NaCl}, 0.3 \% \mathrm{CaCl}_{2}$. Core had been shut-in for six days to allow gelation.

Viscosity of first pore volume of effluent during brine injection was $8.8 \mathrm{cp} @ 11 \mathrm{~s}^{-1}$.

\begin{tabular}{|c|c|c|c|}
\hline Flux & Injected & \multicolumn{2}{|c|}{ Residual Resistance Factors } \\
\hline$(\mathrm{ft} / \mathrm{d})$ & Pore Volumes & Segment 1 & Segment 2 \\
\hline 0.222 & 2.2 & --- & -- \\
\hline 0.834 & 1.6 & 48 & 6.5 \\
\hline 2.22 & 1.2 & 22.9 & 3.3 \\
\hline 8.34 & 2.2 & 14.1 & 3.1 \\
\hline 22.2 & 5.3 & 8.2 & 2.6 \\
\hline 83.4 & 7.4 & 4.6 & 2.2 \\
\hline 222 & 34.0 & 3.2 & 1.9 \\
\hline 83.4 & 6.5 & 3.6 & 2.0 \\
\hline 22.2 & 5.8 & 4.9 & 2.3 \\
\hline 8.34 & 4.1 & 7.4 & 2.6 \\
\hline 2.22 & 2.7 & 26.0 & 3.4 \\
\hline 0.834 & 0.4 & 54.6 & 4.7 \\
\hline 0.222 & 1.7 & 109 & 7.1 \\
\hline & $\overline{75.1}$ & & \\
\hline
\end{tabular}


TABLE 16

Rheology of 3000-ppm Xanthan (without $\mathrm{Cr}^{3+}$ ) in Berea Sandstone with a Residual Oil (n-dodecane) Saturation

Brine permeability (prior to oil saturation) $=531 \mathrm{md}$. $\phi=0.207 . k_{\mathrm{ocw}}=350 \mathrm{md} . k_{\mathrm{wro}}=193 \mathrm{md} . \mathrm{S}_{\mathrm{or}}=0.31$.

Total core length $=15.24 \mathrm{~cm}$, diameter $=1.27 \mathrm{~cm}$.

Segment 1 length $=2.54 \mathrm{~cm}$, segment 2 length $=12.7 \mathrm{~cm}, 85^{\circ} \mathrm{F}$.

(a) Resistance factors during injection of 3000-ppm xanthan, $3 \% \mathrm{NaCl}, 0.3 \% \mathrm{CaCl}_{2}$.

\begin{tabular}{|c|c|}
\hline $\begin{array}{l}\text { Flux } \\
(\mathrm{ft} / \mathrm{d})\end{array}$ & $\begin{array}{l}\text { Injected } \\
\text { Pore Volumes }\end{array}$ \\
\hline 223 & 134.0 \\
\hline 83.8 & 18.4 \\
\hline 22.3 & 14.9 \\
\hline 8.38 & 5.3 \\
\hline 2.23 & 2.7 \\
\hline 0.838 & 6.6 \\
\hline 2.23 & 2.6 \\
\hline 8.38 & 3.0 \\
\hline 22.3 & 7.1 \\
\hline 83.8 & 34.5 \\
\hline 223 & 39.5 \\
\hline 83.8 & 11.7 \\
\hline 22.3 & 10.7 \\
\hline 8.38 & 5.6 \\
\hline 2.23 & 2.1 \\
\hline 0.838 & 5.3 \\
\hline 2.23 & 2.3 \\
\hline 0.223 & 3.7 \\
\hline 0.0838 & 0.8 \\
\hline & $\overline{310.8}$ \\
\hline
\end{tabular}

\begin{tabular}{|c|c|}
\hline \multicolumn{2}{|c|}{ Resistance Factors } \\
\hline Segment 1 & Segment 2 \\
\hline 4.6 & 6.3 \\
\hline 5.5 & 7.7 \\
\hline 8.1 & 10.3 \\
\hline 12.3 & 14.4 \\
\hline 26.8 & 28.8 \\
\hline 58.3 & 50.0 \\
\hline 31.8 & 30.4 \\
\hline 14.7 & 15.8 \\
\hline 8.9 & 10.9 \\
\hline 5.9 & 7.9 \\
\hline 4.8 & 6.6 \\
\hline 5.7 & 7.7 \\
\hline 8.2 & 10.5 \\
\hline 12.5 & 14.4 \\
\hline 25.9 & 27.6 \\
\hline 47.1 & 46.7 \\
\hline 24.8 & 25.8 \\
\hline 114 & 91.6 \\
\hline 170 & 160 \\
\hline
\end{tabular}

(b) Residual resistance factors during injection of brine with $3 \% \mathrm{NaCl}, 0.3 \% \mathrm{CaCl}_{2}$.

$\begin{array}{cc}\begin{array}{c}\text { Flux } \\ (\mathrm{ft} t / \mathrm{d})\end{array} & \begin{array}{c}\text { Injected } \\ \text { Pore Volumes }\end{array} \\ 223 & 52.8 \\ 83.8 & 14.0 \\ 22.3 & 10.2 \\ 8.38 & 6.9 \\ 2.23 & 0.7 \\ 8.38 & 2.2 \\ 22.3 & 5.2 \\ 83.8 & 3.3 \\ 223 & 36.4 \\ 0.838 & 0.4 \\ & \text { Total: } \quad \frac{132.1}{}\end{array}$

Residual Resistance Factors

Segment 1

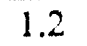

Segment 2

1.2

1.9

$1.3 \quad 2.0$

$1.8 \quad 2.3$

$2.1 \quad 2.9$

$\begin{array}{ll}1.9 & 2.4\end{array}$

$1.4 \quad 2.1$

$1.3 \quad 2.0$

$\begin{array}{ll}1.5 & 2.1\end{array}$

$3.7 \quad 3.2$ 
TABLE 17

Rheology of 3000-ppm Xanthan with 90-ppm $\mathrm{Cr}^{3+}$

in Berea Sandstone with a Residual Oil ( $n$-dodecane) Saturation

Brine permeability (prior to oil saturation) $=531 \mathrm{md}$.

$\phi=0.207 . k_{\text {ocw }}=350$ md. $k_{w r o}=193 \mathrm{md}$. $S_{o r}=0.31$.

Total core length $=15.24 \mathrm{~cm}$, diameter $=1.27 \mathrm{~cm}$.

Segment 1 length $=2.54 \mathrm{~cm}$, segment 2 length $=12.7 \mathrm{~cm}, 85^{\circ} \mathrm{F}$.

(a) Resistance factors during injection of 3000-ppm xanthan, $90-p p m \mathrm{Cr}^{3+}, 3 \% \mathrm{NaCl}, 0.3 \% \mathrm{CaCl}_{2}$.

\begin{tabular}{|c|c|c|c|c|}
\hline \multirow{2}{*}{$\begin{array}{l}\text { Flux } \\
(\mathrm{ft} / \mathrm{d})\end{array}$} & \multirow{2}{*}{$\begin{array}{l}\text { Injected } \\
\text { Pore Volumes }\end{array}$} & \multicolumn{2}{|c|}{ Resistance Factors } & \multirow{2}{*}{$\begin{array}{l}\text { Time since gelant } \\
\text { formulation (hrs) }\end{array}$} \\
\hline & & Segment 1 & Segment 2 & \\
\hline 223 & 23.0 & 5.7 & 7.1 & 1.2 \\
\hline 83.8 & 19.1 & 6.7 & 8.3 & 1.8 \\
\hline 22.2 & 5.6 & 9.7 & 10.4 & 2.6 \\
\hline 8.38 & 2.4 & 14.3 & 15.0 & 3.5 \\
\hline 2.22 & 1.1 & 26.4 & 25.7 & 5.3 \\
\hline 0.838 & 0.3 & 49.0 & 45.0 & 6.3 \\
\hline
\end{tabular}

(b) Residual resistance factors during injection of brine with $3 \% \mathrm{NaCl}, 0.3 \% \mathrm{CaCl}_{2}$. Core had been shut-in for six days to allow gelation.

Viscosity of first pore volume of effluent during brine injection was $10.6 \mathrm{cp} @ 11 \mathrm{~s}^{-1}$.

\begin{tabular}{cccc}
$\begin{array}{c}\text { Flux } \\
(\mathrm{ft} / \mathrm{d})\end{array}$ & $\begin{array}{c}\text { Injected } \\
\text { Pere Volumes }\end{array}$ & & \multicolumn{2}{c}{$\begin{array}{c}\text { Residual Resistance Factors } \\
\text { Segmenj 1 }\end{array}$} & $\begin{array}{c}\text { Segment 2 } \\
0.838\end{array}$ & 6.8 & 6.0 & 6.5 \\
2.23 & 3.3 & 5.0 & 4.9 \\
8.38 & 4.9 & 3.0 & 3.7 \\
22.3 & 9.6 & 2.4 & 3.1 \\
83.8 & 12.6 & 1.9 & 2.4 \\
223 & 74.2 & 1.5 & 1.9 \\
83.8 & 15.4 & 1.4 & 2.0 \\
22.3 & 8.8 & 1.5 & 2.1 \\
8.38 & 4.9 & 2.0 & 2.4 \\
2.23 & 1.7 & 2.4 & 2.8 \\
0.838 & 5.7 & 3.4 & 3.4 \\
& Total: $\quad \frac{57.9}{147.9}$ & &
\end{tabular}

* Value at the end of 6.8 pore volumes. 


\section{Rheology During Gelation}

Researchers at the University of $\mathrm{Kansas}^{66,67}$ have examined the flow of $\mathrm{Cr}^{3+}$-xanthan gelants in sandpacks. They believe that filtration of polymer aggregates plays an important role in permeability reduction during gel treatments. ${ }^{66,68}$ A. filtration mechanism is most likely to be important if flow is occurring at the time when polymer aggregates grow to the size of pore throats. However, early in the gelation process (when polymer aggregates are small or are present in very low concentrations), filtration may not be important. Our resistance-factor data show that, early in the gelation process, the rheology of $\mathrm{Cr}^{3+}$-xanthan gelants is not significantly different from that of a xanthan solution without $\mathrm{Cr}^{3+}$. Data from the University of Kansas do not contradict this finding. ${ }^{66,67}$

How long after the gelant is prepared does the filtration mechanism become important? Our data indicate that the rheology in porous media remains unchanged for at least half of the time required for the onset of gelation to be noticed in a beaker or viscometer. Data from the literature (see Table 18) suggest that even longer time periods may be required for the filtration mechanism to be important for $\mathrm{Cr}^{3+}$-xanthan gelants. (The data listed in Table 18 refer to gelants that contain 1500-ppm xanthan, 50-ppm $\mathrm{Cr}^{3+}$.)

Shutting in wells after gelant injection is a common practice. Therefore, if the well is shut in relatively early in the gelation process, a filtration mechanism may not be important.

If flow is still occurring at the time when significant polymer aggregates develop, of course, filtration may be important. During the filtration process, will polymer aggregates plug lowpermeability zones to a greater extent than high-permeability zones? Intuitively, we expect the answer to this 4 , lestion to be "yes." Consistent with intuition, Hejri et al ${ }^{66}$ noted that resistance factors for $\mathrm{Cr}^{3+}$-xanthan polymer aggregates increased with decreasing permeability. More work is needed to fully assess the impact of filtration on the effectiveness of gel treatments.

\section{Effect of Flow Through Porous Media on Gelation Time}

We noted that flow through Berea at high rates $(225 \mathrm{ft} / \mathrm{d})$ decreased the gelation time for a geiant that contained 3000 -ppm xanthan and $90-p p m \mathrm{Cr}^{3+}$. In contrast, Hejri et al..$^{66}$ and Garver et $a l .{ }^{42}$ found that flow through sandpacks and Berea sandstone increased the gelation time for more dilute $\mathrm{Cr}^{3+}$-xanthan gelants. Hejri et al. used gelants with 1500-ppm xanthan and 50-ppm $\mathrm{Cr}^{3+}$, while Garver et al. used gelants with 500-ppm to 1000-ppm xanthan and with 50-ppm to 150-ppm $\mathrm{Cr}^{3+}$. Interestingly, Jousset et al ${ }^{67}$ found cases where flow through sandpacks did not change the gelation time significantly for a gelant that contained $1500-\mathrm{ppm}$ xanthan and $50-\mathrm{ppm} \mathrm{Cr}^{3+}$. Clearly, more work is needed to resolve how flow through porous media affects gelation. 
TABLE 18

Gelation Onset for $\mathrm{Cr}^{3+}-$ Xanthan Gelants

(1500-ppm xanthan, 50-ppm $\mathrm{Cr}^{3+}$ )

Time until onset of gelation noticed in a viscometer

$$
\begin{aligned}
& 11-17 \text { hours } \\
& 11-17 \text { hours } \\
& 11-17 \text { hours } \\
& 11-17 \text { hours } \\
& 11-17 \text { hours } \\
& 11-17 \text { hours }
\end{aligned}
$$

Time until onset of gelation noticed in eorous media

50 hours

45 hours

18 hours

33 hours

21 hours

28 hours

\section{Literature source}

Figs. $2 \& 4$ of Ref. 66

Figs. $2 \& 6$ of Ref. 66

Figs. 2 \& 3 of Ref. 67

Figs. $2 \& 7$ of Ref. 67

Figs. $2 \& 8$ of Ref. 67

Figs. $2 \& 9$ of Ref. 67

\section{Rheology During Brine Injection After Gelation}

Our most disturbing finding was that residual resistance factors were very low during brine injection after gelation (see Tables $13 \mathrm{~b}, 15 \mathrm{~b}$, and $17 \mathrm{~b}$ ). In general, residual resistance factors for the $\mathrm{Cr}^{3+}$-xanthan gels were not much greater than those for xanthan solutions without $\mathrm{Cr}^{3+}$ (compare with Tables 12b, 14b, and 16b).

Why were residual resistance factors so low? We note that the pH of freshly prepared $\mathrm{Cr}^{3+}$ $x a n t h a n$ gelant was 3.8. We also note that if $\mathrm{pH}$ was maintained at a value of 7 (using buffers), gelation did not occur. Reservoir rocks usually have a tremendous capacity to buffer aqueous solutions near neutral $\mathrm{pH}^{38,47-50}$ Therefore, contact with reservoir rock could render $\mathrm{Cr}^{3+}$-xanthan gelants ineffective by changing $\mathrm{pH}$ and inhibiting gelation.

Do our low residual resistance factors contradict previous literature reports? Several researchers ${ }^{33,66,67}$ noted substantial residual resistance factors for $\mathrm{Cr}^{3+}$-xanthan gels (as high as 9200). These researchers of ten used lower polymer and $\mathrm{Cr}^{3+}$ concentrations (e.g., 1500-xanthan, 50-ppm $\mathrm{Cr}^{3+}$ ) than those used in our study. Thus, our low residual resistance factors appear surprising upon first consideration. However, the previous researchers performed their studies using sandpacks (with Ottawa sand). These sandpacks do not contain clays or carbonate minerals (e.g., dolomite, calcite, siderite) that are responsible for the buffering capacity of reservoir rocks. Therefore, it is not surprising that gelation in these sandpacks is not inhibited to the extent observed in consolidated sandstones.

In order to achieve higher residual resistance factors, additional core experiments were performed using a gelant that ccntained 4000-ppm xanthan and $154-\mathrm{ppm} \mathrm{Cr}^{3+}$. The brine for these experiments contained $0.5 \% \mathrm{KCl}$, and the temperature was $105^{\circ} \mathrm{F}\left(41^{\circ} \mathrm{C}\right)$. The viscosity of this brine was $0.65 \mathrm{cp}$ at $105^{\circ} \mathrm{F}$. After saturation with brine and determination of permeabilities and porosities, tracer studies were performed to determine dispersivity values $\left(\alpha_{i}\right)$ for the cores. Gelant was then placed in the cores using a flux of $15.7 \mathrm{ft} / \mathrm{d}$. The cores were then shut in for several days to allow gelation to occur. After gelation, brine was injected to determine residual resistance factors. Finally, 
tracer studies were again performed to determine the final dispersivity values $\left(\alpha_{f}\right)$ and the relative changes in pore volume $\left(\mathrm{V}_{\mathrm{pf}} / \mathrm{V}_{\mathrm{pi}}\right)$ that were caused by the gel.

One core experiment (in 77-md Berea) was performed using a $\mathrm{Cr}^{3+}$-xanthan gelant without adjustment of $\mathrm{pH}$. This gelant had an initial pH value of 4 . The gelation time for this gelant (at $105^{\circ} \mathrm{F}$ ) was six hours, and a rigid gel was formed. However, we noted that this gelant had very little buffering capacity (i.e., its $\mathrm{pH}$ could be changed very easily). During injection of gelant at $\mathrm{pH}=4$, we also noted that the $\mathrm{pH}$ of the effluent remained near neutral after several pore volumes. We were concerned that a pH value of 4 was not representative of the $\mathrm{pH}$ experienced by the gelant in the core. Therefore, we performed two core experiments (in 66-md Berea and in 483-md Berea) in which the $\mathrm{pH}$ was 7 for the in jected gelant.

Residual resistance factors after gel placement are shown in Figs. 18-20 and Tables 19-21 for three different Berea cores. In all three cores, residual resistance factors decreased significantly with increased flux. Equations that describe the relation between residual resistance factor $\left(F_{r r}\right)$ and flux $(\mathrm{u}$, in $\mathrm{ft} / \mathrm{d})$ are included in rigs. 18-20. Power-law exponents for the relations vary from -0.29 to -0.55 . Of course, brine is a Newtonian fluid, so the apparent "shear-thinning" behavior must be attributed to the gel in the core rather than to the brine. The apparent shear-thinning behavior generally is not due to gel mobilization upon exposure to successively higher flow rates. Stable pressure drops were quickly achieved at a given flux. Also, residual resistance factors at a given flux in a given core were fairly reproducible (see Tables 19-21). (Again, these tables list data in the chronological order in which they were collected.)

We noted that $\mathrm{Cr}^{3+}$-xanthan gelants that were buffered at $\mathrm{pH}=7$ did not appear to gel during beaker tests. Based on this observation we expected to see low residual resistance factors for the gelants that were injected at $\mathrm{pH}=7$. Thus, we were somewhat surprised that residual resistance factors for gelant at $\mathrm{pH}=7$ were generally higher than those for gelant at $\mathrm{pH}=4$ (except at high flux values).

Figures $21-23$ show results of tracer studies that were performed before and after gel placement in the three cores, respectively. (The tracer bank contained potassium iodide that was monitored spectrophotometrically at $230 \mathrm{~nm}$.) In all three cores, the gel increased dispersivity values (by factors ranging from 5.5 to 17.8). The $50 \%$ concentration level for tracer effluent provides an indication of the fraction of original pore volume $\left(\mathrm{V}_{\mathrm{pf}} / \mathrm{V}_{\mathrm{pi}}\right)$ that remains after gelation. This fraction ranged from 0.55 to 1 . In 77 -md Berea, the gel formed from gelant at $\mathrm{pH}=4$ apparently occupies $45 \%$ of the original pore volume (see Fig. 21). In contrast, in 483-md Berea, the gel formed from gelant at $\mathrm{pH}=7$ apparently occupies an insignificant fraction of the original pore volume (see Fig. 23). How can a gel reduce permeability by a factor ranging from 50 to 714 (Table 21) and yet occupy a very small fraction of the pore volume? Perhaps, small gel particles lodge in pore throats-thereby, dramatically reducing brine permeability without occupying much volume. We hope to address this question in more detail during some of our future studies with $\mathrm{Cr}^{3+}-\mathrm{xanthan}$ gels and gelants. 

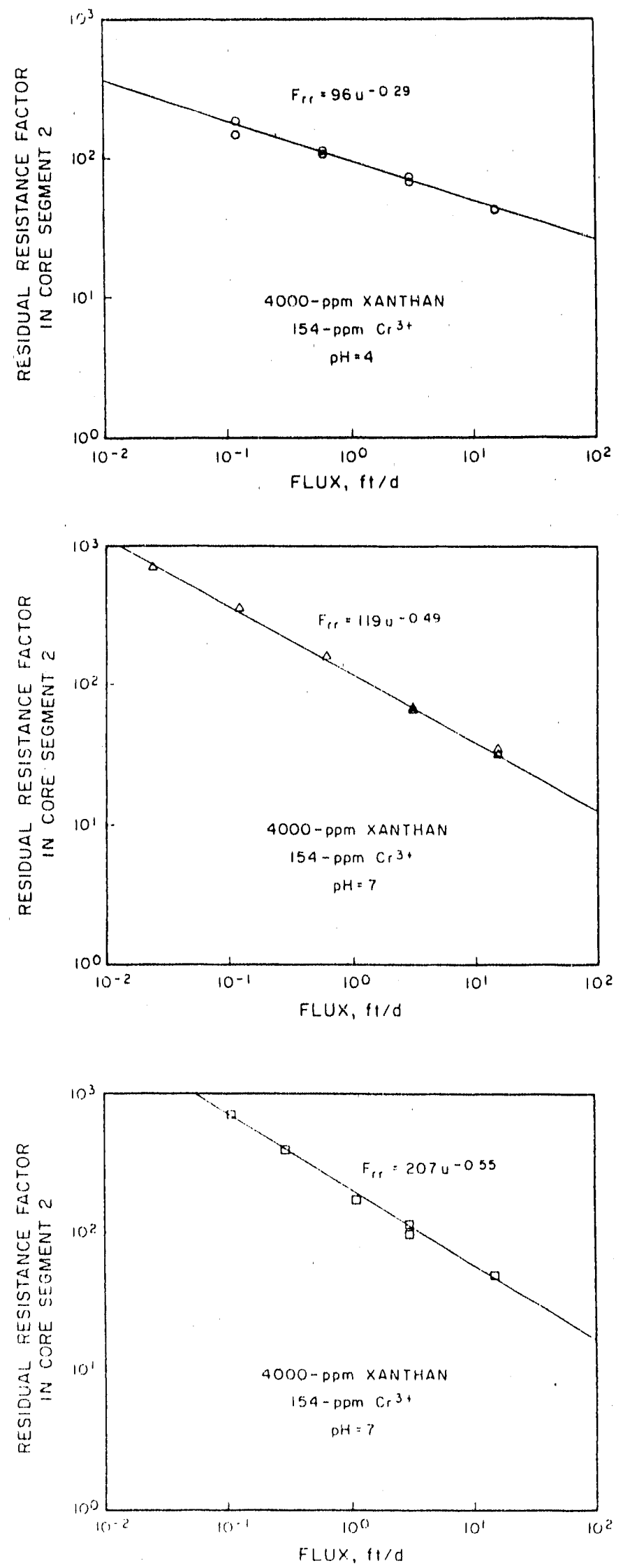

Fig. 18. Residual Resistance Factor vs. Flux in 77-md Berea. Initial Gelant $\mathrm{pH}=4$.

Fig. 19. Residual Resistance Factor vs. Flux in 66-md Berea. Initial Gelant $\mathrm{pH}=7$.

\section{Fig. 20. Residual Resistance Factor vs. Flux in 483-md Berea. Initial Gelant $\mathrm{pH}=7$.}


TABLE 19

Residual Resistance Factors After Placement and Gelation in 77-md Berea Sandstone, $\phi=0.184$. Initial gelant $\mathrm{pH}=4$.

4000-ppm Xanthan, 154-ppm $\mathrm{Cr}^{3+}, 0.5 \% \mathrm{KCl}, \mathrm{pH}=4,105^{\circ} \mathrm{F}$. Total core length $=13.44 \mathrm{~cm}$, diameter $=3.57 \mathrm{~cm}$.

Segment 1 length $=2.44 \mathrm{~cm}$, segment 2 length $=11.4 \mathrm{~cm}$. Dispersivity before gel placement $\left(\alpha_{i}\right)=0.210 \mathrm{~cm}$. Dispersivity after gel placement $\left(\alpha_{f}\right)=1.163 \mathrm{~cm}$. $\alpha_{i} / \alpha_{r}=5.54$.

Fraction of original pore volume left after gelation $=0.55$

\begin{tabular}{cc}
$\begin{array}{c}\text { Flux } \\
(\mathrm{ft} / \mathrm{d})\end{array}$ & $\begin{array}{c}\text { Residual resistance factor } \\
\text { in core segment2 }\end{array}$ \\
\hline 0.126 & 190 \\
0.628 & 115 \\
3.14 & 68 \\
15.7 & 43 \\
3.14 & 74 \\
0.628 & 109 \\
0.126 & 151 \\
15.7 & 42
\end{tabular}


TABLE 20

Residual Resistance Factors After Placement and Gelation in 66-md Berea Sandstone, $\phi=0.185$. Initial gelant $\mathrm{pH}=7$.

4000-ppm Xanthan, 154-ppm $\mathrm{Cr}^{3+}, 0.5 \% \mathrm{KCl}, \mathrm{pH}=7,105^{\circ} \mathrm{F}$. Total core length $=14.32 \mathrm{~cm}$, diameter $=3.57 \mathrm{~cm}$.

Segment 1 length $=1.69 \mathrm{~cm}$, segment 2 length $=12.63 \mathrm{~cm}$.

Dispersivity before gel placement $\left(\alpha_{i}\right)=0.157 \mathrm{~cm}$.

Dispersivity after gel placement $\left(\alpha_{f}\right)=2.534 \mathrm{~cm}$.

$\alpha_{i} / \alpha_{f}=16.2$.

Fraction of original pore volume left after gelation $=0.84$

Flux Residual resistance factor

(ft/d)

in core segment 2

$\begin{array}{cr}15.7 & 34 \\ 3.14 & 67 \\ 15.7 & 31 \\ 3.14 & 65 \\ 0.628 & 157 \\ 0.025 & 690 \\ 0.126 & 348 \\ 15.7 & 31\end{array}$

TABLE 21

Residual Resistance Factors After Placement and Gelation in 483-md Berea Sandstone, $\phi=0.219$. Initial gelant $\mathrm{pH}=7$.

4000-ppm Xanthan, 154-ppm Cr${ }^{3+}, 0.5 \% \mathrm{KCl}, \mathrm{pH}=7,105^{\circ} \mathrm{F}$. Total core length $=14.44 \mathrm{~cm}$, diameter $=3.57 \mathrm{~cm}$.

Segment 1 length $=1.74 \mathrm{~cm}$, segment 2 length $=12.7 \mathrm{~cm}$.

Dispersivity before gel placement $\left(\alpha_{i}\right)=0.088 \mathrm{~cm}$.

Dispersivity after gel placement $\left(\alpha_{\mathrm{f}}\right)=1.566 \mathrm{~cm}$.

$\alpha_{i} / \alpha_{f=17.8}$

Fraction of original pore volume left after gelational

Flux

$(f / d)$

3.10

1.161

0.310

0.116

15.48

3.10
Residual resistance factor

in core segment 2

116

175

400

714

50

99 

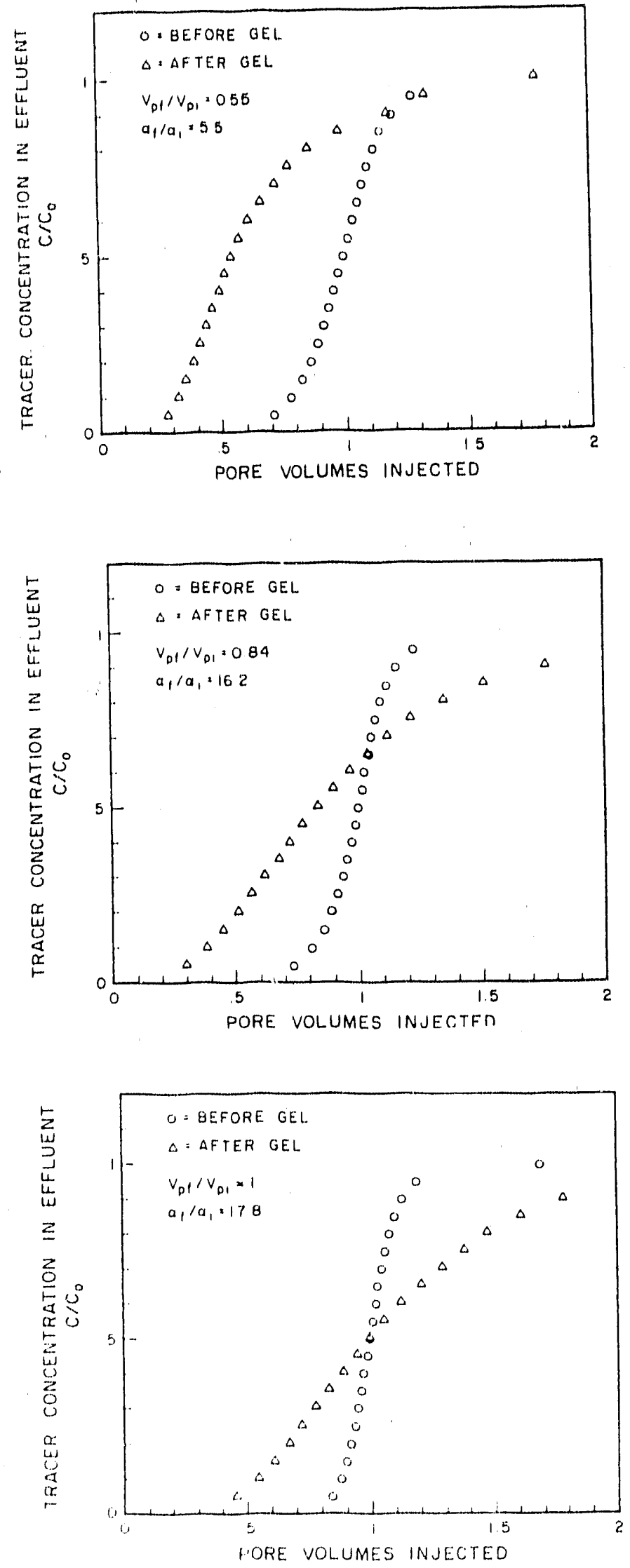

Fig. 21. Tracer Effluent Curves During Brine Injection Before vs. After Gel Placement in 77-md Berea. Initial Gelant $\mathrm{pH}=4$.
Fig. 22. Tracer Effluent Curves During Brine Injection Before vs. After Gel Placement in 66-md Berea. Initial Gelant $\mathrm{pH}=7$.
Fig. 23. Tracer Effluent Curves During Brine Injection Before vs. After Gel Placement in 483-md Berea. Initial Gelant $\mathrm{pH}=7$. 


\section{Example Calculations for an Unfractured Injection Well}

The previous information can be used to estimate the effects of a gel rreatment in an unf ractured injection well. For example, let's consider a reservoir with two noncommunicating layers. Layer 1 has a permeability $\left(k_{1}\right)$ of 483 md and a porosity $\left(\phi_{1}\right)$ of 0.219 . Layer 2 has a permeability $\left(k_{2}\right)$ of $66 \mathrm{md}$ and a porosity $\left(\phi_{2}\right)$ of 0.185 . The wellbore radius $\left(\mathrm{r}_{0}\right)$ is $0.5 \mathrm{ft}$. The pressure drop (downhole) between this unfractured injection well and a nearby production well (in a five-spot pattern) is maintained constant at 1000 psi.

Let $r_{\mathrm{pm}}$ be defined as the greatest radius from the injection well to which gel will ultimately penetrate into the most-permeable layer. During water injection before the gel treatment, the total pressure drop $\left(\Delta P_{f}\right)$ between the injection well and the production well in layer 1 can be separated into two components,

$$
\Delta P_{l}=\Delta P_{w 1}+\Delta P_{w 1}
$$

where $\Delta \mathrm{P}_{\mathrm{w1}}$ is the pressure drop in layer 1 between the injection well and $\mathrm{r}_{\mathrm{pm}}$, and $\Delta \mathrm{P}_{\mathbf{1}}$ is the pressure drop between $r_{p m}$ and the production well. Let $\Psi_{1}$ be defined by Eq. 9 .

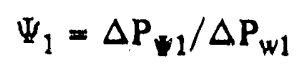

Because of the way in which $\Psi$ is defined, we know that

$$
\Delta P_{\mathfrak{t}}=\left(\Psi_{1}+1\right) \Delta P_{w 1}
$$

During water injection before the gel treatment, we will assume that $\Psi_{1}=\Psi_{2}=2$. See Refs. 24 and 25 for further explanation of $\Psi$.

Prior to the gel treatment, we will assume that the volume within 50 feet from the injection well has been swept to the same residual (irreducible) oil saturation in both layers. Thus, only water is flowing between the injector and $r_{\mathrm{pm}}$. So,

$$
\Delta P_{t}=\left(\Psi_{1}+1\right)\left[\mathrm{q}_{1} /\left(2 \pi h_{1}\right)\right]\left(\mu_{w} / k_{1}\right)\left[\ln \left(r_{p m} / r_{0}\right)\right]
$$

We know that the fluid flux $\left(u_{1}\right)$ at the wellbore sand face in layer 1 is related to injection rate in layer $1\left(\mathrm{q}_{1}\right)$ by

$$
u_{1}=q_{1} /\left(2 \pi h_{1} r_{0}\right)
$$

So, Eq. 11 becomes

$$
\Delta P_{1}=\left(\Psi_{1}+1\right)\left(u_{1} r_{0}\right)\left(\mu_{w} / k_{1}\right)\left[\ln \left(r_{p m} / r_{o}\right)\right]
$$

Solving for $u_{1}$ and substituting appropriate values, we find that

$$
\begin{gathered}
u_{1}=(1000 / 14.7) /[(2+1)(0.5)(12)(2.54)(0.65 / 0.483) \ln (50 / 0.5)] \\
u_{1}=0.24 \mathrm{~cm} / \mathrm{s} \text { or } 680 \mathrm{ft} / \mathrm{d} .
\end{gathered}
$$

In a similar way, the flux $\left(u_{2}\right)$ at the wellbore sand face in layer 2 is found to be $93.4 \mathrm{ft} / \mathrm{d}$ during brine injection prior to the gel treatment. 
A gelant with $4000-p p m$ xanthan and $154-\mathrm{ppm} \mathrm{Cr}^{3+}$ at $\mathrm{pH}=7$ will be injected (without isolating zones) until the gelant reaches a radius $\left(r_{p 1}\right)$ of 50 feet from the injector in layer 1. (So, $r_{\mathrm{pm}}=50 \mathrm{ft}$.) We assume that chemical retention and inaccessible pore volume are the same in $483-\mathrm{md}$ rock as in 66-md rock. We also neglect diffusion and dispersion. (For a discussion of the impact of chemical retention, inaccessible pore volume, diffusion, and dispersion, see Refs. 24-26.)

We can estimate the minimum depth of penetration of gelant in layer 2 . The minimum penetration $\left(r_{p 2}\right)$ into layer 2 will occur if the resistance factor $\left(F_{r}\right)$ of the gelant is equal to one. ${ }^{24,25}$ When $F_{r}=1$, Eq. 15 in Ref. 24 simplifies to

$$
r_{p 2}=\left[\left(\phi_{1} / \phi_{2}\right)\left(k_{2} / k_{1}\right)\left(r_{p 1}{ }^{2}-r_{o}{ }^{2}\right)+r_{0}{ }^{2}\right]^{0.5}
$$

Thus, the minimum value for $r_{\mathrm{p} 2}$ is

$$
r_{p 2}=\left[(0.219 / 0.185)(66 / 483) \times\left(50^{2}-0.5^{2}\right)+0.5^{2}\right]^{0.5}=20.2 \mathrm{ft}
$$

In reality, a viscous xanthan gelant will penetrate a greater distance into layer $2 .{ }^{25}$ However, we will use the optimistic value of $20.2 \mathrm{ft}$ in our subsequent calculations.

After injecting the gelant, the well is shut in to allow gelation. When brine injection is resumed, the total pressure drop between the injector and the production well can be separated into three components. In layer 1 ,

$$
\Delta P_{1}=\Delta P_{p 1}+\Delta P_{w 1}+\Delta P_{w 1}
$$

In Eq. 17, $\Delta \mathrm{P}_{\mathrm{p} 1}$ is the pressure drop across the gel bank in layer 1, i.e., between the injector and the outer radius $\left(r_{p 1}\right)$ of the gel bank in layer 1. Also, $\Delta P_{w 1}$ is the pressure drop between $r_{p 1}$ and $r_{p m}$. This term can be evaluated using Eq. 18.

$$
\Delta \mathrm{P}_{\mathrm{w} 1}=\left(\mathrm{u}_{1} \mathrm{r}_{\mathrm{o}}\right)\left(\mu_{\mathrm{w}} / \mathrm{k}_{1}\right)\left[\ln \left(\mathrm{r}_{\mathrm{pm}} / \mathrm{r}_{\mathrm{p} 1}\right)\right]
$$

In layer 1 this term is equal to zero since $r_{p 1}=r_{p m}$. The term $\Delta P_{\Psi 1}$ in $E q .17$ can be evaluated using Eq. 19.

$$
\Delta P_{\Psi 1}=(\Psi)\left(u_{1} r_{o}\right)\left(\mu_{w} / k_{1}\right)\left[\ln \left(r_{p m} / r_{0}\right)\right]
$$

Substituting appropriate values into Eq. 19 gives

$$
\Delta P_{\Downarrow 1}=u_{1}(2)(0.5)(12)\{2.54)(0.65 / 0.483)[\ln (50 / 0.5)]=378 u_{1} \text { atm. }
$$

( $u_{1}$ must be in units of $\mathrm{cm} / \mathrm{s}$.)

In Eq. 17, $\Delta \mathrm{P}_{\mathrm{pl}}$ can be evaluated using Eq. 21.

$$
\Delta P_{p 1}=u_{1} r_{0}\left(\mu_{w} / k_{1}\right) \int_{r o}^{r p l}\left(F_{r r} / r\right) d r
$$

From Figs. 18-20, we note that the residual resistance factor $\left(F_{r r}\right)$ has the form

$$
F_{r r}=K u^{n}
$$


In radial flow, the flux $(u)$ at any radius $(r)$ in layer 1 can be related to the flux at the wellbore sand face $\left(u_{1}\right)$ in layer 1 .

$$
u=u_{1} r_{0} / r
$$

Therefore, Eqs. 21-23 can be combined and integrated to give Eq. 24.

$$
\Delta P_{p 1}=\left(u_{1}\right)^{(1+n)} r_{0}\left(\mu_{w} / k_{1}\right)(-K / n)\left[\left(r_{p 1} / r_{0}\right)^{-n}-1\right]
$$

From Fig. 20, we know that $F_{r r}=207 u^{-0.55}$ in 483 -ma berea (where $u$ has units of $f t / d$ ). By substituting the appropriate values into Eq. 24 , we find

$$
\begin{aligned}
& \Delta P_{p 1}=\left(u_{1}\right)^{(1-0.55)}[86400 / 12 / 2.54]^{-0.55}(0.5)(12)(2.54)(0.65 / 0.483)(207 / 0.55)\left[(50 / 0.5)^{0.55}-1\right] \\
& \Delta P_{p 1}=1129 u_{1}^{0.45} \mathrm{~atm} .
\end{aligned}
$$

Combining Eqs. 17, 18, 20 and 25 leads to Eq. 26.

$$
(1000 / 14.7)=1129 u_{1}^{0.45}+0+378 u_{1}
$$

Solving Eq. 26 by iteration shows that during brine injection into layer 1 after the gel treatment, $u_{1}$ equals $1.90 \times 10^{-3} \mathrm{~cm} / \mathrm{s}$ or $5.38 \mathrm{ft} / \mathrm{d}$. We note that $5.38 \mathrm{ft} / \mathrm{d}$ is within the range where the $F_{\mathrm{rr}}$ relation is valid in Fig. 20. If $u_{1}$ had been too high, unrealistic $F_{r r}$ values might have been predicted. In particular, $F_{r r}$ values can not be less than one. Therefore, when making calculations such as those shown here, checks should be made to insure that $F_{r r}$ values are realistic.

Finally, the gel treatment is found to reduce brine injectivity in layer 1 to $0.79 \%$ of the original injectivity. This value is determined by dividing $u_{1}$ obtained after the gel treatment ( 5.38 $\mathrm{ft} / \mathrm{d})$ by $\mathrm{u}_{1}$ obtained before the gel treatment $(680 \mathrm{ft} / \mathrm{d})$.

A similar procedure can be used to find the injectivity loss in layer 2 (the 66-md layer). In layer 2, the equation that is analogous to Eq. 18 gives

$$
\Delta P_{w 2}=u_{2}(0.5)(12)(2.54)(0.65 / 0.066)[\ln (50 / 20.2)]=135.7 u_{2} \text { atm. }
$$

The equation that is analogous to Eq. 19 gives

$$
\Delta P_{\Psi_{2}}=u_{2}(2)(0.5)(12)(2.54)(0.65 / 0.066)[\ln (50 / 0.5)]=1376 u_{2} \text { atm, }
$$

From Fig. 19, we know that $F_{\mathrm{rr}}=119 \mathrm{u}^{-0.49}$ in 66 -md Berea (where $u$ has units of $\mathrm{ft} / \mathrm{d}$ ). Thus, the equation that is analogous to Eq. 24 gives

$$
\begin{aligned}
& \Delta \mathrm{P}_{\mathrm{p} 2}=\left(\mathrm{u}_{2}\right)^{(1-0.49)}[86400 / 12 / 2.54]^{-0.49}(0.5)(12)(2.54)(0.65 / 0.066)(119 / 0.49)\left[(20.2 / 0.5)^{0.49}-1\right] \\
& \Delta \mathrm{P}_{\mathrm{p} 2}=3778 \mathrm{u}_{2}^{0.51} \mathrm{~atm} .
\end{aligned}
$$

Summing Eqs. 27, 28, and 29, and equating to the total pressure drop yields

$$
(1000 / 14.7)=3778 u_{2}^{0.61}+135.7 u_{2}+1376 u_{2}
$$

Solving Eq. 30 by iteration shows that during brine injection into layer 2 af ter the gel tre tment, $u_{2}$ equals $3.73 \times 10^{-4} \mathrm{~cm} / \mathrm{s}$ or $1.06 \mathrm{ft} / \mathrm{d}$. We note that $1.06 \mathrm{ft} / \mathrm{d}$ is within the range where the $F_{r r}$ relation 
is valid in Fig. 19. Thus, the gel treatment is found to reduce brine injectivity in layer 2 to $1.13 \%$ of the original in jectivity. This value is determined by dividing $u_{2}$ obtained af ter the gel treatment ( 1.06 $\mathrm{ft} / \mathrm{d})$ by $\mathrm{u}_{2}$ obtained before the gel treatment $(93.4 \mathrm{ft} / \mathrm{d})$. This estimate of injectivity reduction is actually optimistic. Recall that we assumed that the gelant would penetrate the minimum distance of $20.2 \mathrm{ft}$ into layer 2 . Since a viscous xanthan gelant will probably penetrate a greater distance into layer 2 , the final brine injectivity in layer 2 will be somewhat less than $1.13 \%$ of the original in jectivity.

The final result of the gel treatment is that the in jection profile has not been improved to any significant extent. Injectivity in both layers has been reduced by about $99 \%$. This injectivity reduction may be desirable in the most-permeable layer, but it would be very harmful in the oilprodustive layer (i.e., layer 2). Thus, zone isolation would be needed during gel placement. This same conclusion is reached if residual resistance factors are assumed to be constant after gelation. Thus, the apparent non-Newtonian behavior of residual resistance factors for this $\mathrm{Cr}^{3+}$-xanthan gelant will not eliminate the need for zone isolation in unfractured injection wells.

The above analysis can be applied in a similar manner to yel placement in vertically fractured injection wells. To some extent, the analysis is simplified in fractured wells because the flow geometry is linear. However, in fractured wells, two other factors should be considered. First, injection of viscous polymer solutions or gels may extend the length of the fracture. Second, a large part of the beneficial effects that are derived from the gel may come from reducing the flow capacity of the fracture rather than from selectively reducing permeabilities in the different strata that are cut by the fracture. We hope to address these considerations in our future work.

\section{Conclusions}

1. For a large fraction of the time prior to gelation, the presence of $90-\mathrm{ppm} \mathrm{Cr}^{3+}$ did not significantly affect the rheology in porous media of a $3000-p p m$ xanthan solution.

2. For gel formulations containing $3000-\mathrm{ppm}$ xanthan and $90-\mathrm{ppm}^{3+}$, residual resistance factors in Berea sandstone were not much greater than those for xanthan solutions without $\mathrm{Cr}^{3+}$. We suspect that clay and carbonate minerals in Berea forced the gelation reaction to occur near neutral pH rather than at the injection $\mathrm{pH}(3.8)$. In beaker tests, $\mathrm{Cr}^{3+}$-xanthan formed rigid gels at $\mathrm{pH} \approx 4$, but geiation was not apparent for the same formulation at $\mathrm{pH}=7$. Previous researchers reported large residual resistance factors for more dilute $\mathrm{Cr}^{3+}$-xanthan gels in clean sandpacks. The discrepancy between our results and those of previous researchers may be explained in that the lack of clays and carbonate minerals in sandpacks may have allowed gelation to occur at low $\mathrm{pH}$ values rather than at neutral $\mathrm{pH}$. Thus, the w.uffering action of reservoir rocks should be considered when evaluating gel performance in the laboratory.

3. Using 4000-ppm xanthan and 154-ppm $\mathrm{Cr}^{3+}$, a rigid gel was formed in a beaker at $\mathrm{pH}=4$, but gelation was not evident at $\mathrm{pH}=7$. Even so, the composition injected at $\mathrm{pH}=7$ provided substantial residual resistance factors $(30-714)$ in Berea sandsione.

4. Tracer studies incicated that the gel (4000-ppm xanthan, 154-ppm $\mathrm{Cr}_{3}$ ) occupied between $0 \%$ and $45 \%$ of the original pore volume, depending on the initial pH of the gelant and the core permeablity. Tracer studies also revealed that the gel increased dispersivity values in Derea by factors renging from 5.5 to 17.8 . 
5. For a gel with $4000-\mathrm{ppm}$ xanthan and $154-\mathrm{ppm} \mathrm{Cr}^{3+}$ at $\mathrm{pH}=7$ in $483-\mathrm{md}$ Berea, residual resistance factors were quite high (50-714) even though tracer studies indicated that the pore volume occupied by the gel was near zero. Perhaps, small gel particles lodge in pore throats-thereby, dramatically reducing brine permeability without occupying muc'n volume.

6. Residual resistance factors provided by gels (4000-ppm xanthan, 154-ppm $\mathrm{Cr}^{3+}$ ) docreased significantly with increased fluid flux and could be described by a power-law relation over the flux range from 0.025 to $16 \mathrm{ft} / \mathrm{d}$.

7. Laboratory measurements of gel properties in 66-md Berea and in 483-md Berea were used during example calculations to show that the apparent "shear-thinning" nature of residual resistance factors will not eliminate the need for zone isolation during gel placement in unf ractured injection wells. 


\section{IMPAC I OF DIFFUSION, DISPERSION, AND VISCOUS FINGERING ON GEL PLACEMENT IN INJECTION WELLS}

At the peak of activity, $35 \%$ of the enhanced oil recovery projects in the United States were polymer projects. ${ }^{5}$ About $60 \%$ of these polymer projects were gel treatments rather than traditional polymer floods. ${ }^{6}$ The objective of gel treatments is to block fractures or watered-out, highpermeability zones such that fluids injected subsequently are more likely to enter and displace oil from other strata. Many gel projects have been very successful. Unfortunately, many other gel projects have been technical failures. One study revealed that less than $45 \%$ of near-wellbore gel treatments were successful. ${ }^{7}$ In part, the sporadic success rate for gel treatments may be due to the way in which the gels were placed in the reservoir. In most cases when gelling agents were injected, zones were not isolated, so the chemicals had access to all open intervals. Much of the gel formulation enters fractures and/or high-permeability streaks. However, some of this fluid penetrates into strata that one does not want to plug. Therefore, a key issue in gel technology is how to place gels in fractures or "thief" zones without damaging oil-productive zones.

Two recent studies 24,2 examined how injection-flow profiles are modified by unrestricted injection of Newtonian and non-Newtonian gelling agents. These studies found:

1. Zone isolation is much more likely to be needed during placement of gels in unf ractured wells than in fractured wells.

2. Productive zones in unfractured wells can be seriously damaged if zones are not isolated during gel placement.

3. If zones are not isolated during gel placement, the minimum penetration into lowpermeability zones can be achieved by using a water-like gelling agent (having a resistance factor equal to one).

4. Compared with water-like gelling agents, the non-Newtonian rheology of existing polymeric gelling agents will not reduce the need for zone isolation during gel placement.

This study explores the influence of diffusion, dispersion, and viscous instabilities during placement of gels to modify injection profiles. In particular, these phenomena are examined to determine whether they can be exploited to optimize gel piacement.

\section{Gelant Penetration in Oll-Productive Strata}

A common misconception in the application of gel treatments is that injected gelling agents will exclusively enter high-permeability, watered-out channels without penetrating to any significant extent into less-permeable, oil-bearing strata. Straightforward applications of the Darcy equation reveal that gelling agents can penetrate tc a significant degree into all open intervals, ${ }^{24,25}$ For example, if a gelant penetraces fifty feer $(: 5.2 \mathrm{~m})$ radially from an injection well into the mostpermeable layer of a multilayer reservoir, then the gelant can propagate at least five feet $(1.5 \mathrm{~m})$ radially into a zone that is 100 rimes less permeable. This can be seen by examining Fig. 24 . Figure 24 plots the depth of penetration (final radius minus wellbore radius) of gelant into a less-permeable zone (layer $2, k_{2}$ ) when the gelant reaches $50 \mathrm{ft}$ into the most-permeable zone (layer $1, k_{1}$ ). (The wellbore radius is $0.5 \mathrm{ft}$, and all layers have the same porosity.) This information is shown for two Newtonian fluids $\left(F_{r^{-}}\right)$and $\left.F_{r}+00\right)$ and two non-Newtonian fluids. The non-Newtonian fluids 


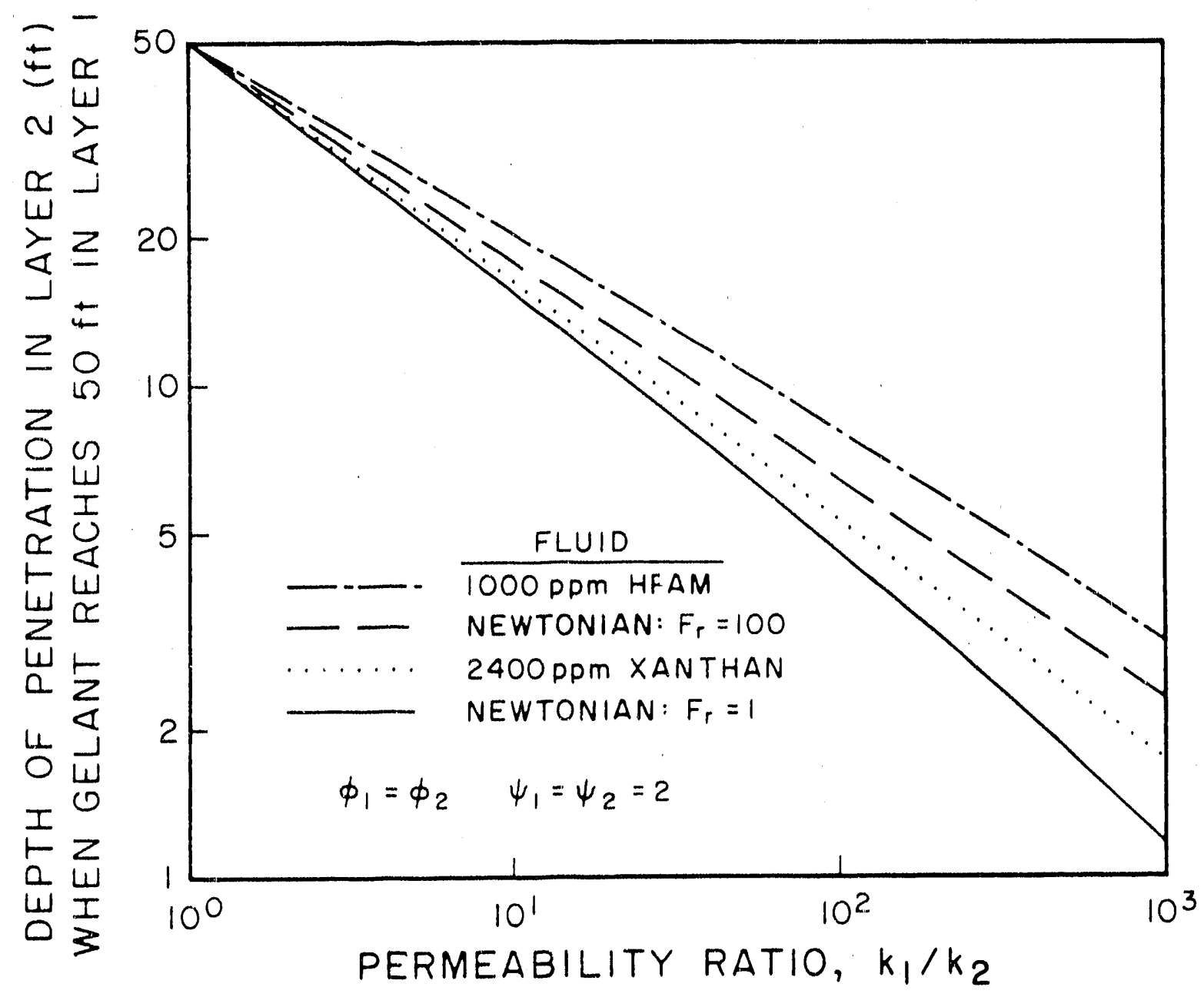

Fig. 24. Relative Penetration of Fluids in an Unfractured Injection Well with Multiple, Noncommunicating Layers. 
included a xanthan solution and a partially hydrolyzed polyacrylamide (HPAM) solution. (Flow properties of the non-Newtonian fluids are described in detail in Ref. 25.) Note that for a given permeability ratio, the three viscous fluids penetrate to a greater depth in the less-permeable layer than does the water-like fluid $\left(\mathrm{F}_{\mathrm{r}}=1\right)$.

The degree of penetration into a given low-permeability zone (relative to that in the mostpermeable zone) increases with increased viscosity (resistance factor) of the injected fluid. ${ }^{24}$ This is a basic principle of polymer flooding and is also evident from Fig. 24. This principle can readily be demonstrated by properly conducting floods in paral'el cores, sandpacks or beadpacks. Table 22 presents results from several parallel floods involving both Newtonian fluids (water and ethanediol) and non-Newtonian fluids (xanthan and HPAM solutions). The floods were performed in parallel linear beadpacks that had a length of $30 \mathrm{~cm}$ and a porosity of $37 \%$. Different-sized beads were packed into clear plexiglass tubes, and clear water was displaced by injecting a dyed fluid. The permeabilities of the beadpacks were 48 darcys and 22 darcys, respectively. The dys and effluent volumes were used to establish the position of the in jectant-water front during the flood. The degree of penetration for each flood $\left(\mathrm{L}_{\mathrm{p} 2} / \mathrm{L}_{\mathrm{p} 1}\right)$ was the distance to which the injectant had propagated into the less-permeable core when the injectant had reached the outlet of the most-permeable core. Table 22 compares experimental values for the degree of penetration with theoretical values determined using the methods described in Refs. 24 and 25. The comparison reveals that the theoretical values closely match the experimental values. If these values had not matched, this would have been cause for concern since the theoretical values are based on nothing more than mass balances and the Darcy equation.

TABLE 22

Results from Parallel Linear Floods

\section{Iniectant}

$1-c p$ dyed water

$11-c p$ ethanediol

2000-ppm xanthan

2000-ppm HPAM

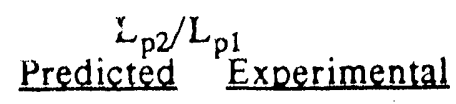

0.46

0.46

0.65

0.67

0.67
0.64

0.66

0.67

Several papers report laboratory results from parallel linear floods involving gelling agents that appear to violate Darcy's law. ${ }^{31,33}$ If these results are accepted without question, then one is misled to believe that the gelling agents only enter the most-permeable porous media and that zone isolation is not needed during field projects. However, caution must be used when performing parallel laboratory floods with gelling agents. In particular, at least two factors must be considered. First, the flow lines leading to the core inlets must be completely filled with gelling agent at the start of the displacement process. Otherwise (if the lines are filled with water instead of gelling agent), the gelling agent could penetrate well into the most-permeable core before the gelling agent could even reach the inlet face of the less-permeable core. Second, the short bank of gelling agent in the lesspermeable core can be difuted enough by diffusion and dispersion to prevent gelation while still allowing gel to form in the most permeable core. As will be discussed later, this is far more likely to occur on a laboratory scale than on a field scale. 
For the calculations represented in Fig. 24, no crossflow occurs between layers. If crossflow can occur between layers or flow paths in a reservoir, viscous gelants will penetrate into lowpermeability layers to a greater extent than if crossflow is not possible. In fact, under some circumstances (if the gelant/water mobility ratio is less than the permeability contrast between adjacent layers), the depth of penetration of gelant in a low-permeability layer can be the same as that in an adjacent high-permeability layer. ${ }^{69,70}$ Thus, if crossflow can occur, viscous gelants can damage oil-productive zones to a greater extent than if crossflow is not possible.

In preparing Fig. 24, diffusion, dispersion, chemical retention, and inaccessible pore volume effects were neglected. The impact of chemical retention and inaccessible pore volume on these calculations has been described previously. ${ }^{24,25}$ The role of diffusion and dispersion will be discussed in this study.

\section{Dilution by Diffusion}

In concept, diffusion and dispersion could dilute gelling agents enough to prevent gelation in less-permeable, oil-productive zones while still allowing a gel plug to form in watered-out, highpermeability streaks. ${ }^{71}$ Whether or not a chemical bank can be diluted enough by diffusion to prevent gelation depends on at least four factors: 1) the size of the chemical bank, 2) the diffusion coefficient, 3) the gelation time, and 4) the extent of dilution required to prevent gelation.

Diffusion coefficients are typically on the order of $10^{-5} \mathrm{~cm}^{2} / \mathrm{s}$ for low-molecular-weight chemicals in water. ${ }^{72}$ These chemicals include gelling agents such as acrylamide monomer, phenol, and formaldehyde. Diffusion coefficients are typically on the order of $10^{-8} \mathrm{~cm}^{2} / \mathrm{s}$ for highmolecular-weight polymeric gelling agents such as polyacrylamide or xanthan. ${ }^{73}$ For low-molecularweight species in a viscous polymer solution (e.g., $\mathrm{Cr}_{2} \mathrm{O}_{7}{ }^{2-}$ in water with 2000 -ppm polyacrylamide), the diffusion coefficient should have some intermediate value-varying inversely with the viscosity of the solution. ${ }^{72}$ The relationship between the apparent diffusion coefficient $(D)$ in porous media and the molecular binary diffusion coefficient $\left(D_{0}\right)$ has been described by

$$
\mathrm{D}=\mathrm{D}_{\mathrm{o}} /(\mathrm{F} \phi)
$$

where $F$ is the formation electrical resistivity and $\phi$ is porosity. Apparent diffusion coefficients in porous media are commonly 20 to 40 percent less than molecular diffusion coefficients. ${ }^{52}$

Gelation times range from a few minutes to several days for most formulations that have been considered for near-wellbore gel treatments. In general, the gelation time decreases with increasing concentrations of the gelling agents. ${ }^{16,17}$ Also, some minimum concentration of the proper reactants must be present in order for gelation to occur. In most field applications of gel treatments, the concentrations of reactants that are in jected are well above the minimum levels required for gelation. Thus, significant dilution (of ten oy a factor of two or more) is required in order to prevent gelation.

For much of this study, we assume that the gelation reaction is stopped by only a ten percent dilution of the reactants. Thus, the reader should bear in mind that the reductions in gel-bank size that are forecasi due to dilution by diffusion and/or dispersion will be overly optimistic. By overestimating the impact of diffusion and dispersion in this analysis, we increase confidence in a major conclusion from this study. That is, in field applications, diffusion and dispersion will not usually cause enough dilution to prevent gelation in the less-permeable zones. 
In field applications of gel treatments, wells are commoniy shut-in for some time after injection of the gelling agent to allow the gel to form. During the time prior to gelation, diffusion acts to dilute the chemical banks (see Fig. 25a). The size of the mixing zone $\left(\mathrm{L}_{\mathrm{m}}\right)$ created by diffusion alone (no dispersion) during this time can be approximated using Eq. 32,

$$
L_{m}=3.62 \sqrt{\mathrm{Dt}_{\mathrm{g}}}
$$

where $t_{g}$ is gelation time. The mixing zone given by Eq. 32 extends from the point where the gelling agent has been diluted to 90 percent of the original concentration to the point where the gelling agent has been diluted to 10 percent of the original concentration. ${ }^{52}$ Figure $25 \mathrm{~b}$ illustrates a typical concentration profile that results when diffusion or dispersion smears an interiace that was originally sharp. Figure $25 \mathrm{~b}$ also illustrates the size of the mixing zone that is given by Eq. 32 .

If the gelation reaction is stopped by a ten percent dilution of the reactants, then diffusion will reduce the gel bank size by the distance $\mathrm{L}_{\mathrm{m}} / 2$. Figure 26 provides values of $\mathrm{L}_{\mathrm{m}} / 2$ as a function of time and diffusion coefficient. A key point illustrated by Fig. 26 is that diffusion will not create a large mixing zone in the period associated with typical gelation times. Even for relatively large diffusion coefficients $\left(10^{-5} \mathrm{~cm}^{2} / \mathrm{s}\right), \mathrm{L}_{\mathrm{m}} / 2$ is only about $0.2 \mathrm{ft}(5 \mathrm{~cm})$ af ter ten days. Considering the depths of penetration for gelling agents in typical field applications (see Fig. 24), diffusion is not likely to have a significant impact on a field scale.

In contrast, diffusion can significantly affect results from parallel laboratory corefloods. Consider injection of a $1-\mathrm{cp}$ gelling agent to displace water from two one-foot-long cores that are being flooded in parallel. Assume that one core is ten times more permeable than the other and both cores have the same porosity. When the gelling agent reaches the outlet of the most-permeable core, the gelling agent will have penetrated $0.1 \mathrm{ft}$ into the less-permeable core. Over the course of one day, most of the gelling agent in the less-permeable core could be diluted if the diffusion coefficient is $10^{-5} \mathrm{~cm}^{2} / \mathrm{s}$.

\section{Dilution by Dispersion}

During injection of a gelant to miscibly displace water, both diffusion and dispersion will occur. While diffusion is the transport of mass because of spatial concentration differences, dispersion is mixing caused by variations in the velocity within each flow channel and from one channel to another. ${ }^{74}$ In flow through reservoirs, dispersion usually is much more important than diffusion. ${ }^{74}$

The concentration gradient at the front between the two fluids will be governed by the value of the Peclet number $\left(N_{p e}\right)$,

$$
N_{p e}=v L / K
$$

where $v$ is interstitial velocity, $L$ is distance traveled by the fluid front, and $K$ is the dispersion coefficient. The longitudinal dispersion coefficient $(K)$ is given by Eq. 34 .

$$
\mathrm{K}=\mathrm{D}+\alpha \mathrm{v}^{\mathrm{B}}
$$

where $\alpha$ is the longitudinal dispersivity and $B$ is an exponent that has been determined by experiment to be in the range from 1 to 1.2 . In this work, a value of 1 will be used for $B$. 


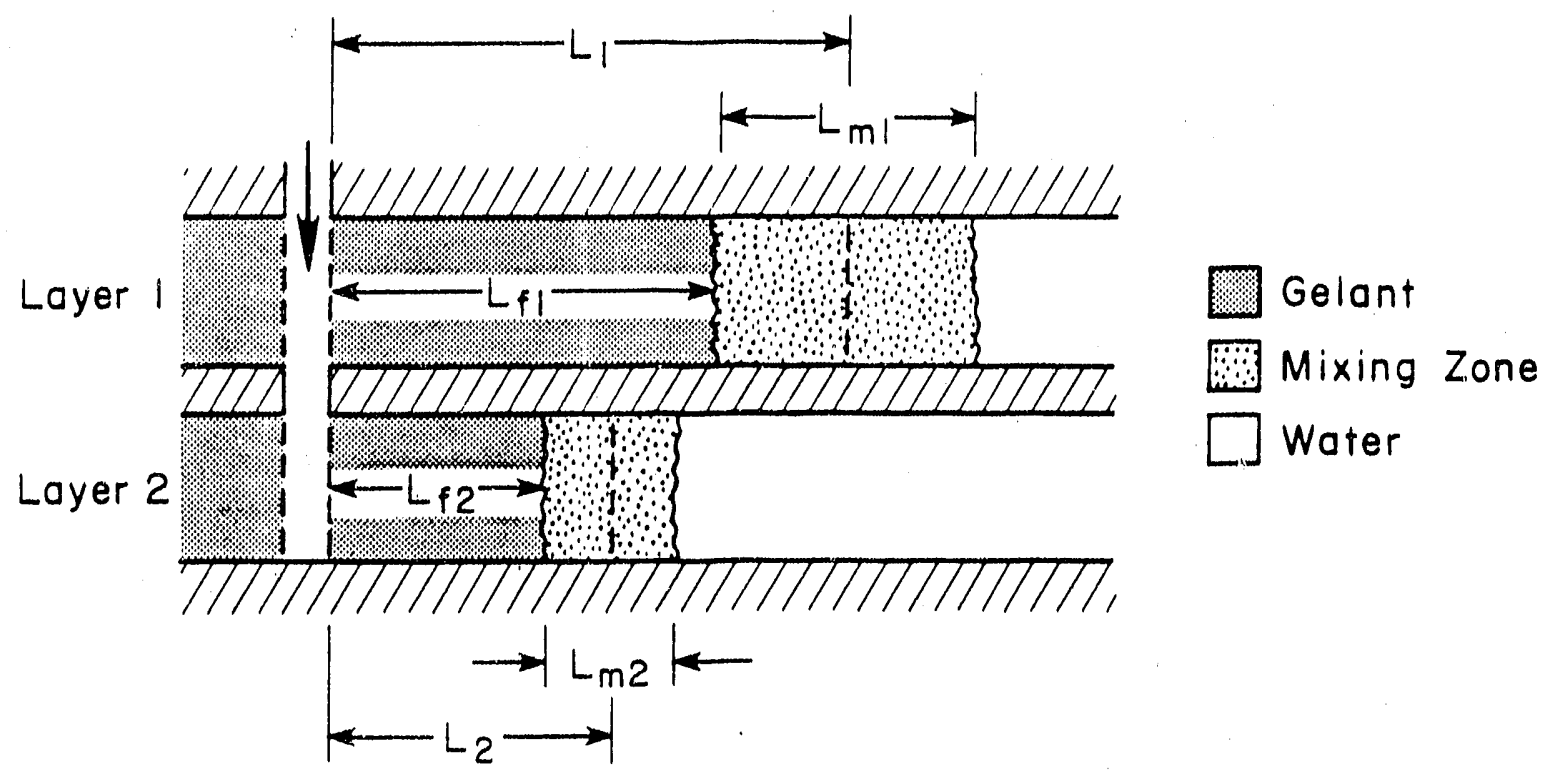

Fig. 25a. Illustration of Mixing Zones

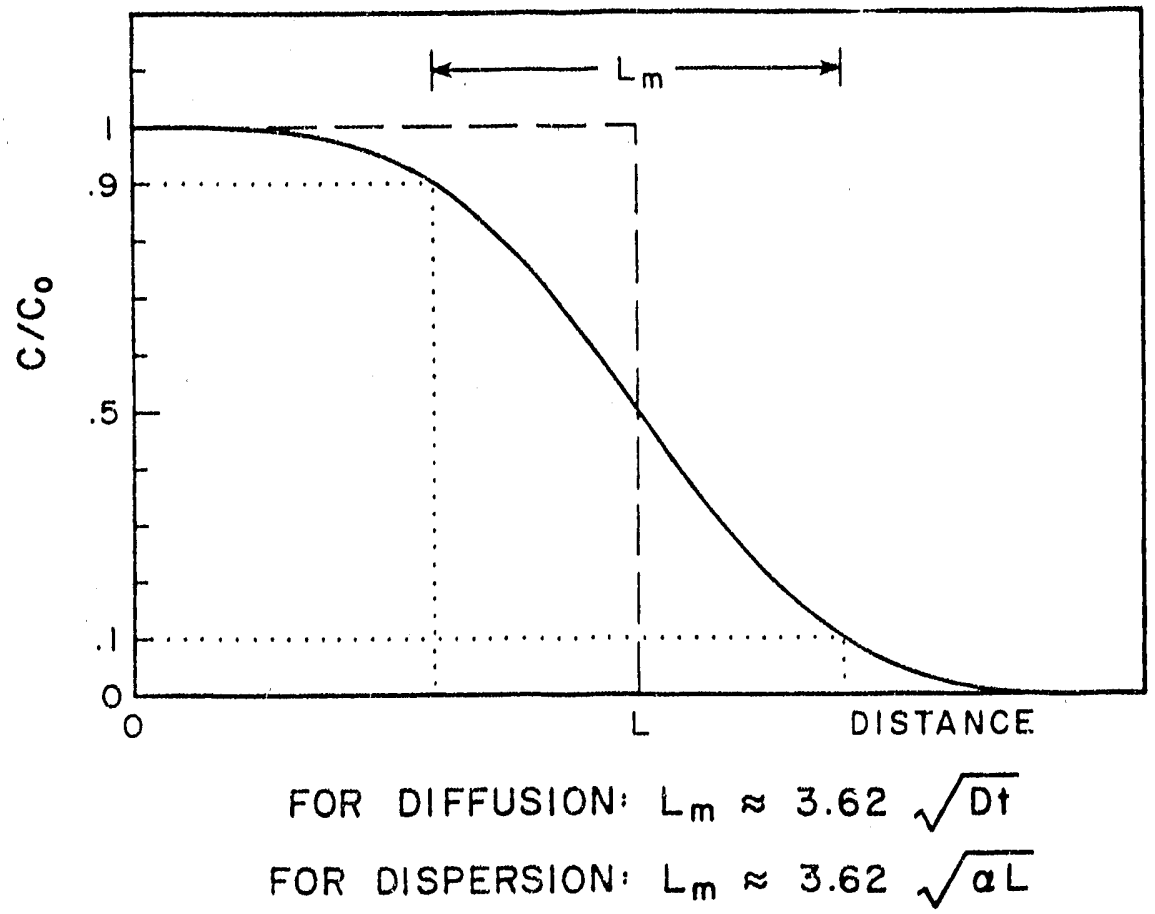

Fig, 25b. Concentration Profile at the Interface Between the Water Bank and the Gelling-Agent Bank. 


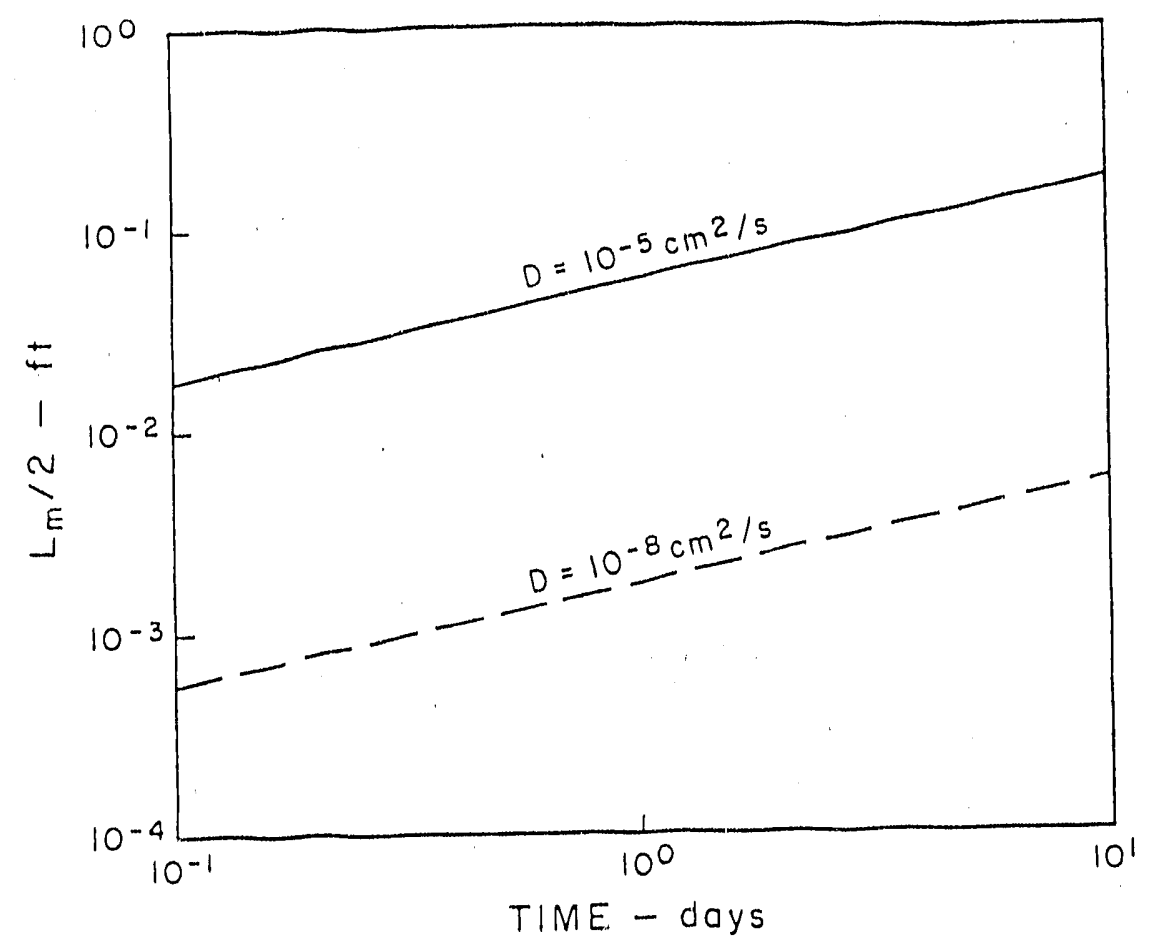

Fig. 26. Length of Mixing Zone Due to Diffusion.

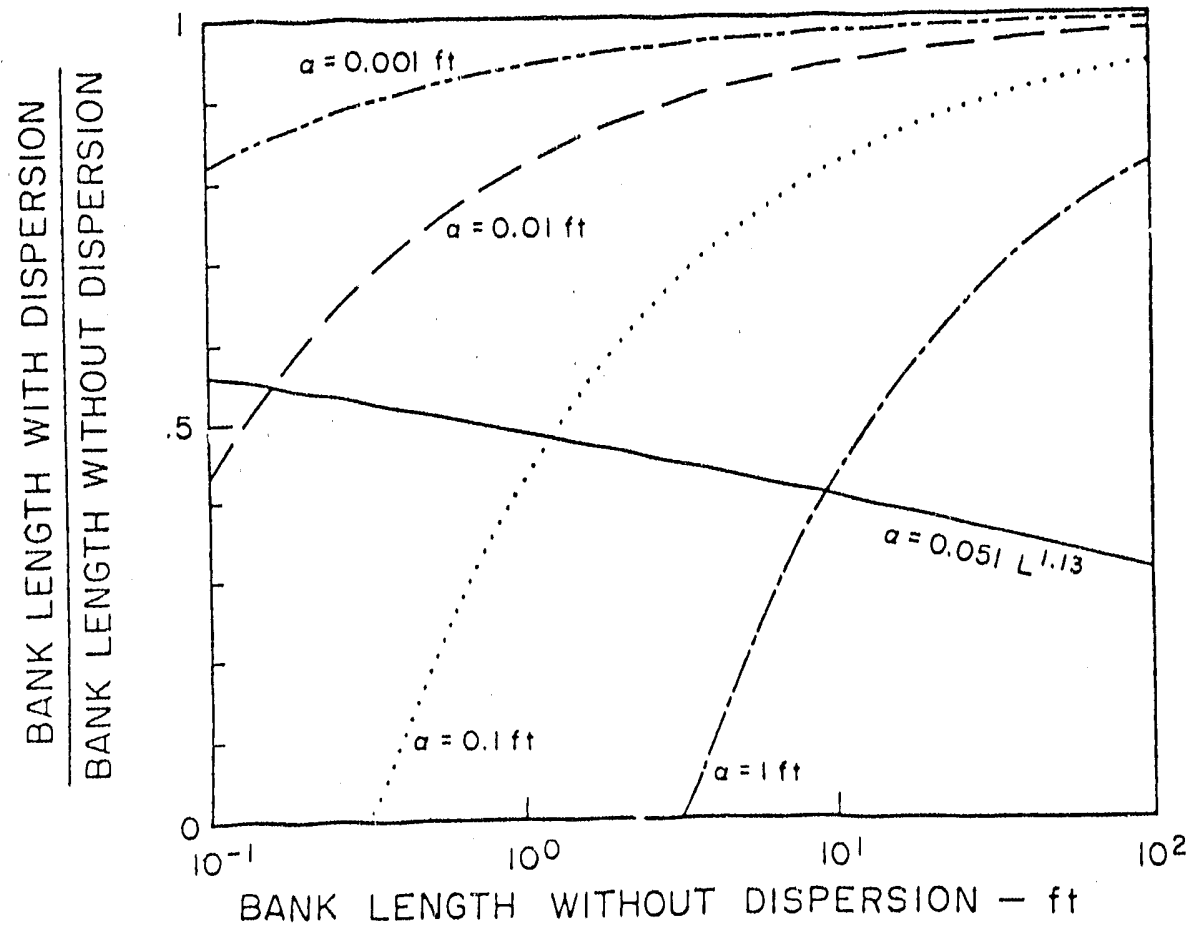

Fig. 27. Effect of Dispersion on Bank Size. 
The size of the mixing zone (again, between the 90\%-10\% concentration levels) created by dispersion can be estimated using Eq. 35 ,

$$
L_{m} \times 3.62 \sqrt{\alpha L}
$$

where $\alpha$ is the dispersivity of the porous medium and $\mathcal{L}$ is the distance traveled by the fluid front. Laboratory values for $\alpha$ commonly are in the range from 0.001 to $0.05 \mathrm{ft}(0.0003$ to $0.015 \mathrm{~m})$. $^{74,75}$ However, field dispersivity values are usually significantly greater than laboratory values because of the greater heterogeneity experieuced on the larger scale. ${ }^{74,76}$ Using over 60 dispersivity values from both field and laboratory measurements, Aiya et al. ${ }^{74}$ noted that the following relation correlates dispersivity values over a wide range of length scales (although there is considerable scatter in the data):

$$
\alpha=0.044 \mathrm{~L}^{1.13}
$$

if $\alpha$ and $L$ are expressed in meters, and

$$
\alpha=0.051 \mathrm{~L}^{1.13}
$$

if $\alpha$ and $L$ are expressed in feet.

The above information can be used to estimate reductions in the size of a gel bank due to dilution by dispersion. These estimates are shown in Fig. 27 as a function of dispersivity and original bank size $(L)$. The size of the gel bank after dispersion $\left(L_{f}\right)$ relative to the original bank size was approximated using

$$
\mathrm{L}_{\mathrm{f}} / \mathrm{L}=\left[1-\mathrm{L}_{\mathrm{m}} /(2 \mathrm{~L})\right]
$$

Four constant-dispersivity cases are shown. These cases indicate that the smallest chemical banks should experience the greatest dilution by dispersion. However, very high near-wellbore dispersivity values $(\sim 1 \mathrm{ft})$ are required to prevent gelation during typical field gel treatments (compare Fig. 27 with Fig. 24).

In contrast to the calculations made using constant dispersivity values, the Arya correlation (Eq. 36b) predicts that the relative reduction in bank size will be fairly insensitive to the length of the original (undiluted) gel bank. In fact, the correlation actually suggests that dispersion has a slightly greater impact as bank size increases (see Fig. 27). Therefore, if the Arya correlation applies, dispersion will not inhibit gel formation in low-permeability zones to a greater extent than in highpermeability zones.

For a given length scale, different strata may exhibit different dispersivities. Thus, in concept, one could exploit a situation where the dispersivity was much higher in the less-permeable layers than in the most-permeabie layers. However, close examination of Fig. 27 suggests that the dispersivity contrast must be very large in order to be exploitable.

Figures 26 and 27 are most applicable for diffusion and dispersion in a linear geometry. Calculation of diffusion and dispersion effects in a radial geometry can be complex. However, the relative reduction in chemical bank size will be less in a radial geometry than in a linear geometry. This can be illustrated using the information in Table 23, which cornpares dispersion predictions in 
linear flow with radial-flow predictions from three different sets of researchers. The relative reduction in bank length (or radius) can be approximated by $\left[1-L_{m} /(2 L)\right]$, where $L_{m} / L$ values are listed in Table 23. The $\alpha / \mathrm{L}$ term in Table 23 is equivalent to the reciprocal of the Peclet number. ${ }^{74}$

TABLE 23

Comparison of Dispersion Predictions for Linear and Radial Flow

(Bank Length with Dispersion)+(Bank Length without Dispersion)

\begin{tabular}{|c|c|c|c|c|}
\hline \multirow[b]{2}{*}{$\alpha / L$} & \multirow[b]{2}{*}{ Linear Elow } & \multicolumn{3}{|c|}{ Radial Flow } \\
\hline & & $\begin{array}{l}\text { Perkins- } \\
\text { Johnston }^{77}\end{array}$ & $\begin{array}{l}\text { Tang- } \\
\text { Peaceman }^{78}\end{array}$ & $\begin{array}{l}\text { Tang- } \\
\text { Babu }^{75}\end{array}$ \\
\hline $1 / 32$ & 0.68 & 0.82 & 0.79 & 0.79 \\
\hline $1 / 16$ & 0.55 & 0.74 & 0.70 & 0.70 \\
\hline $1 / 8$ & 0.36 & 0.63 & 0.56 & -- \\
\hline $1 / 4$ & 0.09 & 0.48 & 0.36 & -- \\
\hline
\end{tabular}

In the discussion to this point, gelation is assumed to be prevented by a $10 \%$ reduction of the original concentration of gelling agent. As mentioned earlier, the impact of diffusion and dispersion is generally overestimated using this assumption. Other values may be used for the concentration below which gelation does not occur. In general, if the minimum concentration for gelation is greater than $50 \%$ of the or:-ginal concentration, then diffusion and dispersion will reduce the size of the gel bank in a given zone. However, if the minimum concentration for gelation is less than 50\% of the original concentration, then the gel bank will be increased in size by the action of diffusion and dispersion. (Of course, the final gel strength in the mixing zone may not be as great as that in the undiluted portion of the gel bank.) To approximate the size of a mixing zone $\left(L_{m}\right)$ whose limits are other than at the $90 \%-10 \%$ concentration levels, the coefficient $(3.62)$ in Eqs. 32 and 35 can be replaced by the appropriate value from Table 24 . These approximations are truly valid only for diffusion or dispersion in a one-dimensional, semi-infinite medium where the concentration gradient is described by the standard error-function solution. ${ }^{52}$ More sophisticated methods ${ }^{53,78}$ may be more appropriate for predicting concentrations in the mixing zone in some cases.

TABLE 24

Mixing-Zone Limits

\begin{tabular}{l} 
Concentration limits \\
of the mixing zone \\
\hline $95 \%-5 \%$ \\
$90 \%-10 \%$ \\
$80 \%-20 \%$ \\
$70 \%-30 \%$ \\
$60 \%-40 \%$ \\
$50 \%-50 \%$
\end{tabular}

\begin{tabular}{l} 
Coefficient in \\
Eas. 32 and 35 \\
\hline 4.65 \\
3.62 \\
2.38 \\
1.48 \\
0.72 \\
0.00
\end{tabular}

The objective of gel trearments is to reduce injectivity in high-permeability watered-out zones while maintaining injectivity in less-permeable oil-productive zones. Figures 28a and 28b compare injectivity behavior for gel placement in an uniractured (radial flow) injection well where the gelling agent is allowed to penetrate $50 \mathrm{ft}$ into the most-permeable layer (layer 1). The gelling agent penetrates into a less-permeable layer (layer 2 ) to a radius determined by the Darcy equation and the rheology of the gelling agent. ${ }^{24,2 S}$ (The particular reservoir model used here corresponds to 
the $\Psi_{1}=2, W_{2}-2$ unfractured in jection well in Refs. 24 and 25.) Diffusion and dispersion are allowed to the extent calculated using Eqs. 32 and 35 , respectively, Various diffusion coefficients and dispersivity values were examined, as indicated in Figs. 28a and 28b. To maximize dilution by diffusion, the gelation time was assumed to be ten days. Wherever gel forms, the permeability to water is assumed to be reduced by a factor of $30\left(F_{\mathrm{rr}}=30\right)$. The Carreau rheological model for a 2400 ppm xanthan solution was used in generating the xanthan curve. To generate the HPAM curve, the Heemskerk dual power-law model for a 1000 -ppm polyacrylamide solution was used. Both of these rheological models are described and illustrated in Ref. 25. For the monomer curves, the resistance factor for the gelling agent had a value of one.

Figures $28 \mathrm{a}$ and $28 \mathrm{~b}$ indicate that for conventional gel treatments in unfractured injection wells, the injectivity (of water af ter the gel treatment) will be reduced to about the same extent in all layers that have permeabilities that are greater than 0.01 times that of the most-permeable layer. By assuming an extremely large near-wellbore dispersivity $(\alpha=1 \mathrm{ft})$, dilution by diffusion and dispersion could eliminate the need for zone isolation during gel placement if the permeability contrast $\left(k_{1} / k_{2}\right)$ was greater than 100:1. However, with more realistic dispersion behavior (i.e., the Arya correlation), diffusion and dispersion will not have a significant impact for permeability contrasts of less than 1000:1.

If diffusion and dispersion are to be exploited to eliminate the need for zone isolation during gel placement, then much smaller gelant banks must be used. Figure 29 provides a means to estimate the maximum allowable depth of penetration of gelant in the most-permeable layer (layer 1) in order for diffusion and dispersion to prevent gelation in a given less-permeable layer (layer 2). This is shown for both linear geometries (e.8., fractured injection wells) and radial geometries (e.8., unf ractured injection wells). The reader should note that Fig. 29 was generated by assuming (1) that a monomeric gelant was used $\left(D=10^{-5} \mathrm{~cm}^{2} / \mathrm{s}, \mathrm{F}_{\mathrm{r}} \mathrm{l}\right)$ and (2) only $10 \%$ dilution is required to prevent gelation. Thus, Fig. 29 tends to overestimate the impact of diffusion and dispersion, especially for polymeric gelants.

Diffusion and dispersion during gel placement in parallel laboratory corefloods can mislead one to conclude that zone isolation is not needed during gel placement in field projects. Fig. 30 shows the fraction of original injectivity retained in cores after gel placement in 1 -ft-long parallel linear corefloods. For core permeability ratios of $10: 1$ or greater, the gelling agent could be diluted sufficiently to prevent gelation in the less-permeable core.

\section{Effect of a Water Postflush on Water-Like Gelant Banks}

Additional mixing and thinning of gelant banks can be induced by injecting water to displace gelants away from the wellbore prior to gelation. The impact of a water postflush will be considered in two parts. In this section the discussion will focus on displacement of a water-like gelant $\left(F_{r}=1\right)$ by injection of water. The next section will discuss the case where water displaces a viscous gelant.

If water is injected to displace a water-like gelant, the mobility ratio for the displacement is unity. In radial flow, in jection of a water postflush will thin the gelant bank, even in the absence of diffusion and dispersion (see Fig. 31). However, this thinning is not large, and it occurs to about the same proportion in all zones. This is illustrated in Fig. 32. The situation represented in Fig. 32 is as follows. First, a water-like gelant is injected into a radial, multilayer reservoir until the gelant propagates to a radius of $50 \mathrm{ft}$ in the most-permeable layer (layer 1). (The wellbore radius is $0.5 \mathrm{ft}$, and all layers have the same porosity,) At this time the length of the gelant bank (bank radius minus the wellbore radius) will be $49.5 \mathrm{ft}, 15.3 \mathrm{ft}, 4.5 \mathrm{ft}$, and $1.2 \mathrm{ft}$ in layers that have permeabilities that are 1,10,100, and 1000 times less than that in the most-permeable layer, respectively. After injec- 

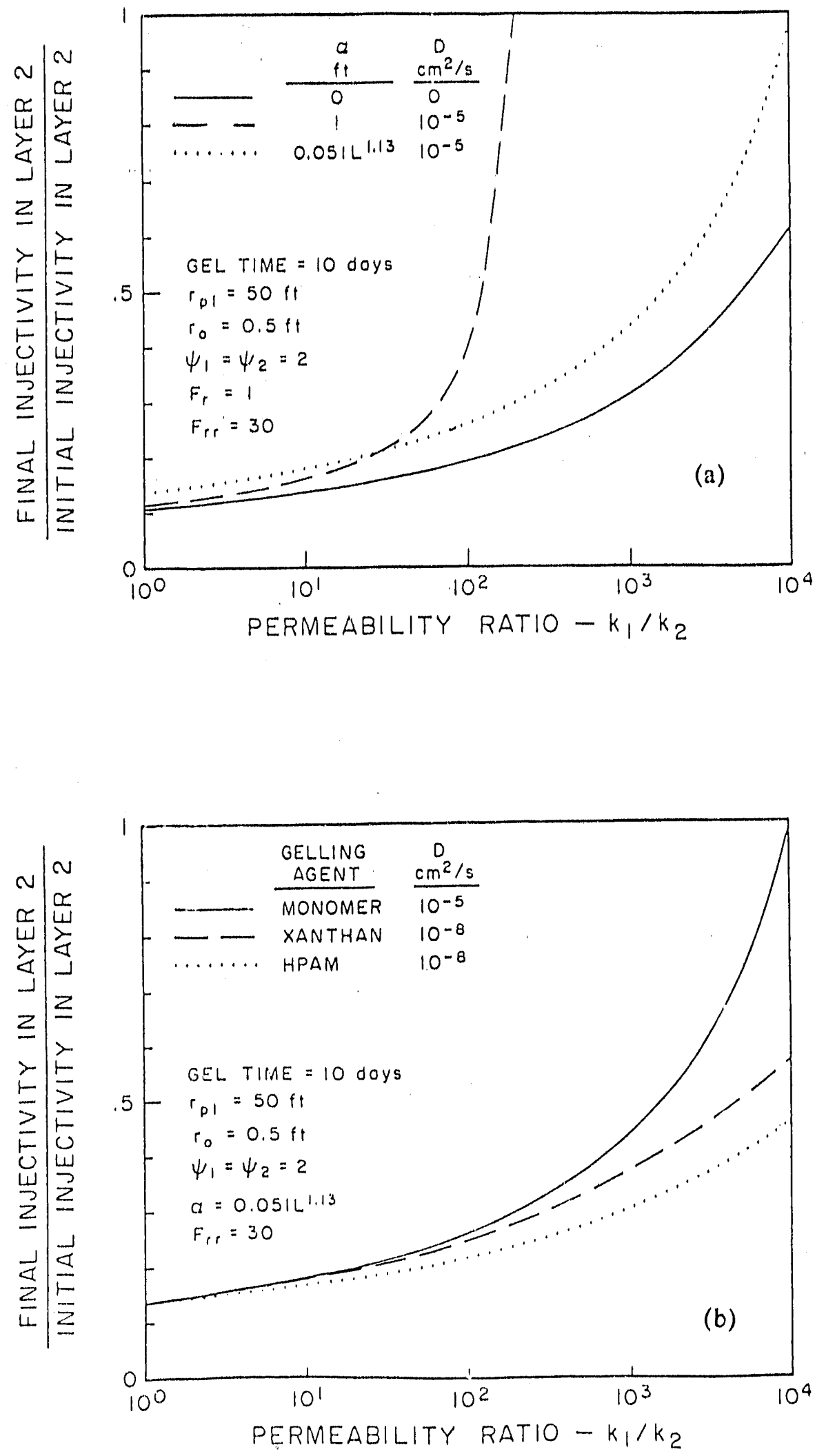

Fig. 28. Impact of Diffusion and Dispersion on Gel in Unfractured Injection Wells. 


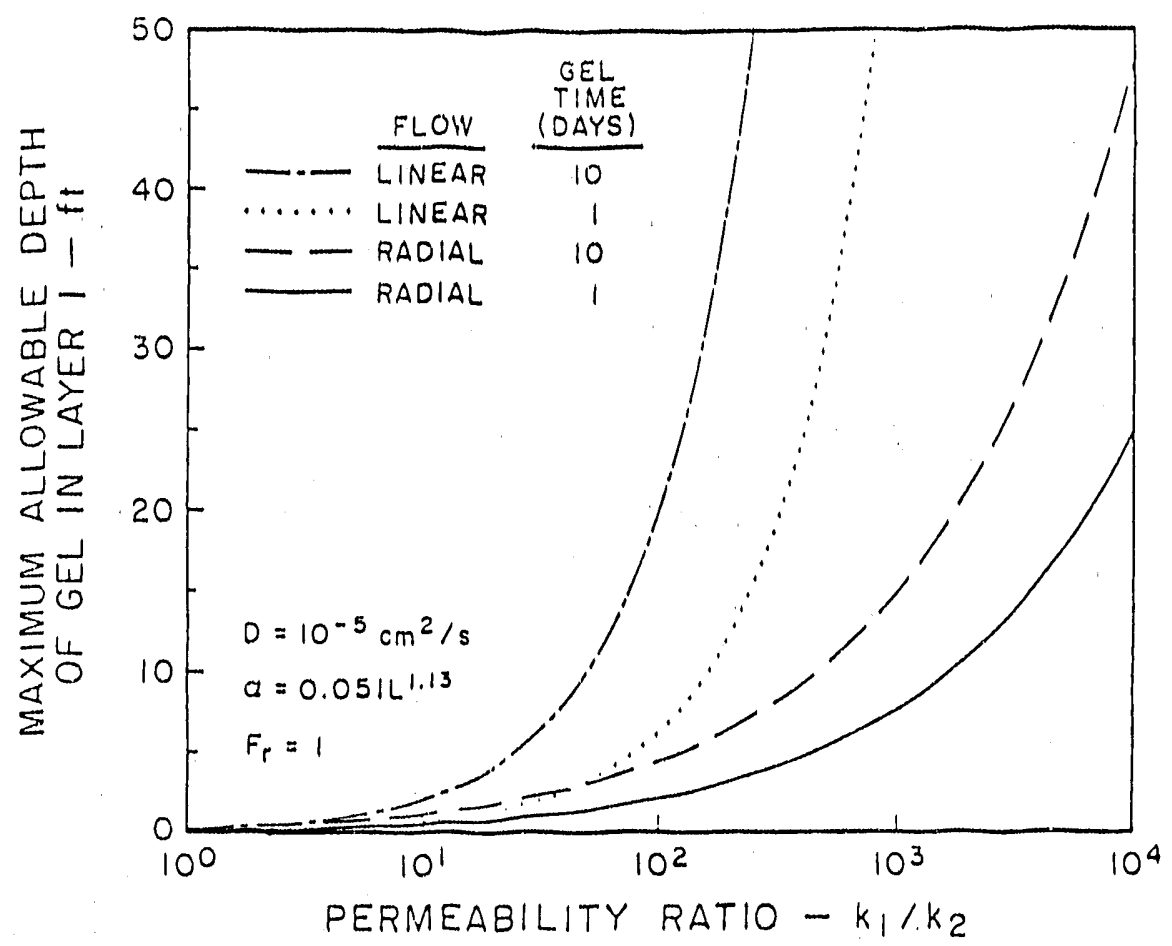

Fig. 29. Exploiting Diffusion and Dispersion to Prevent Gelation in Layer 2.

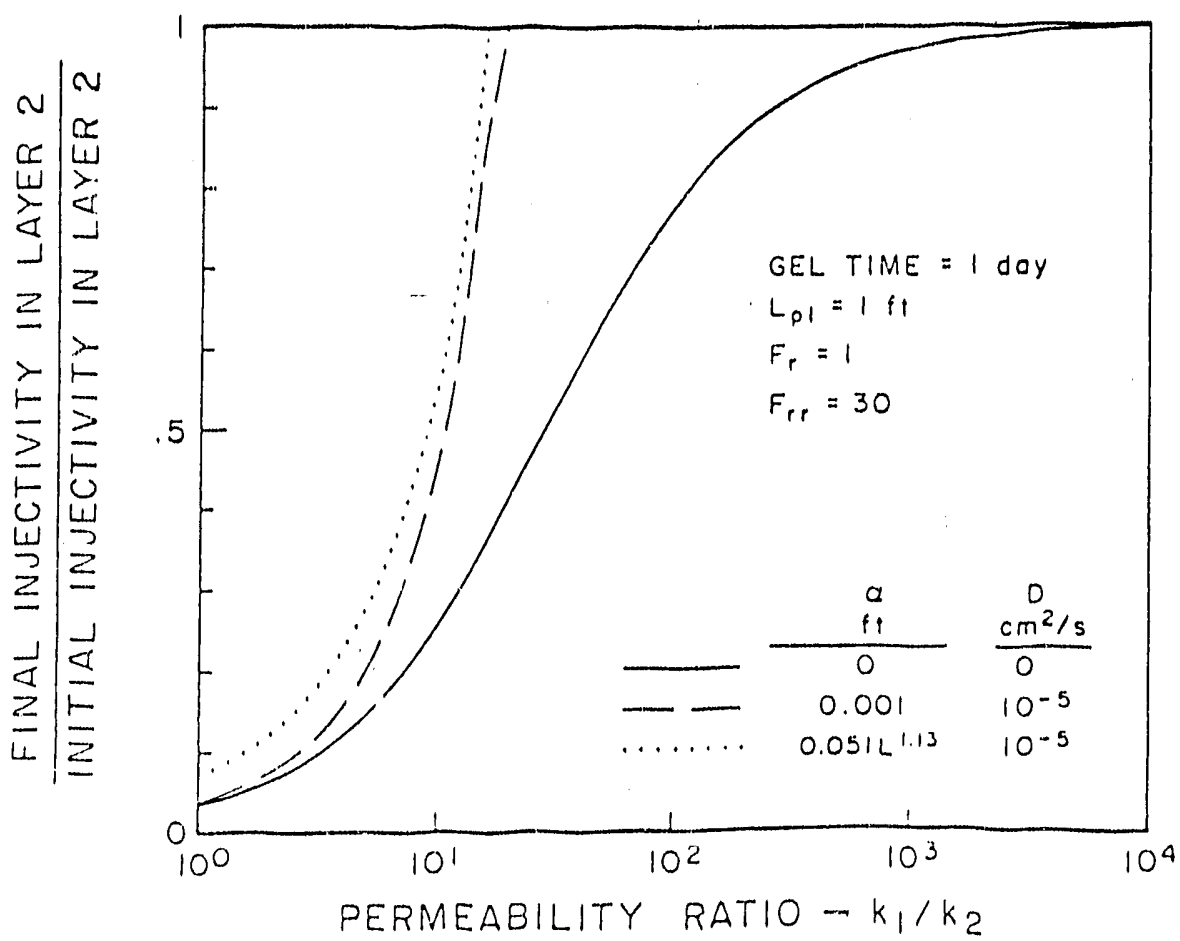

Fig. 30. Impact of Diffusion and Dispersion during Parallel Linear Corefloods. 


\section{WILL A WATER POST FLUSH REDUCE}

THE NEED FOR ZONE ISOLATION?

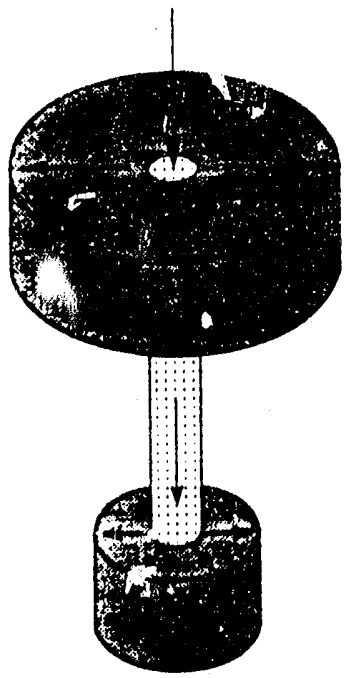

(a)

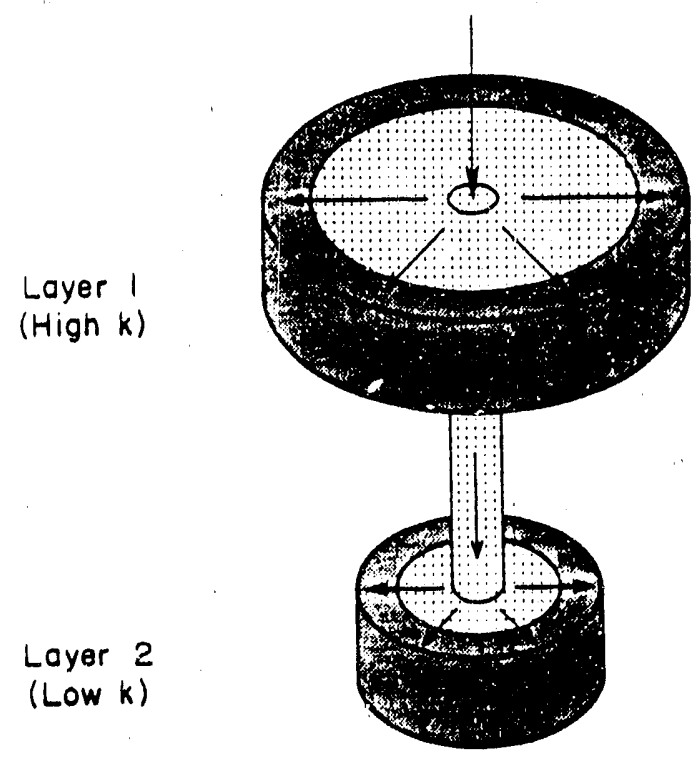

(b)

Gelant Woter

Fig. 31. Illustration of a Water Postflush.

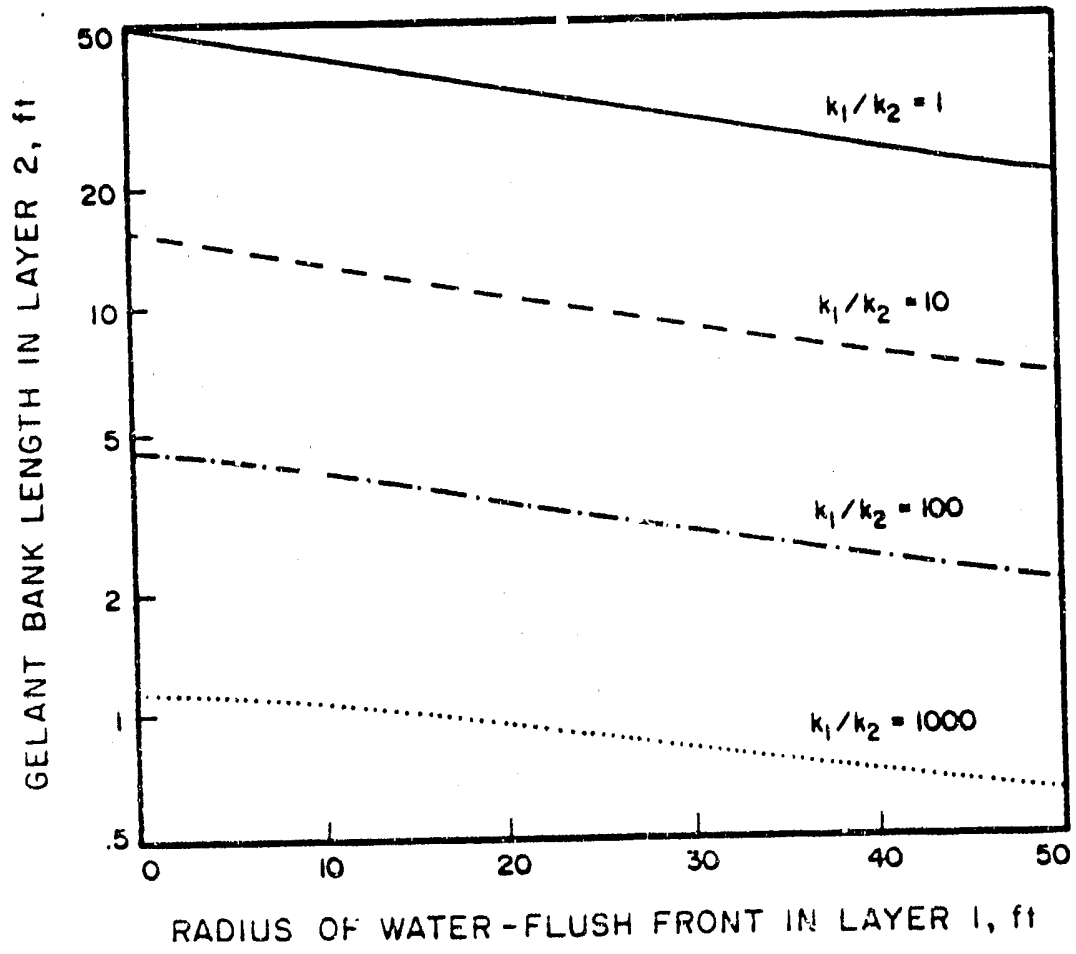

Fig. 32. Thinning of the Gelant Bank during a Water Postflush in Radial Flow. 
tion of the gelant, water is injected to displace the gelant away from the wellbore. Figure 32 plots the length of the gelant bank in a given zone (layer 2 , where the permeability ratis,,$k_{1} / k_{2}$, is specified in the figure) as a function of the radius of the water postflush in the most-permeable zone. Figure 32 reveals that a water postflush out to $50 \mathrm{ft}$ in the most-permeable zone redusts the length of the gelant bank in all zones by roughly a factor of two.

The objective of gel treatments is to reduce injectivity in high-permeability watered-out zones while maintaining injectivity in less-permeable oil-productive zones. The effect of a water postflush on injectivities is shown in Fig. 33. Four cases are illustrated. In all four cases, a water-like $\left(F_{r}=1\right)$ gelant was allowed to penetrate $50 \mathrm{ft}$ radially into the most-permeable layer. The gelant penetrated to some lesser radius in a given less-permeable layer. In two cases, dispersion was allowed to occur during fluid injection. The dispersivity was given by Eq. 36b. Diffusion was also allowed to occur at the gelant-water interface (prior to gelation). The diffusion coefficient was $10^{-5} \mathrm{~cm}^{2} / \mathrm{s}$, and the gelation time was one day. In the other two cases, no diffusion or dispersion was allowed.

In two cases illustrated in Fig. 33, a water pustflush was injected (prior to gelation) to displace the inner radius of the gelant bank to $50 \mathrm{ft}$ from the wellbore in the most-permeable layer. Of course, the postflush had a smaller radius in a given less-permeable layer. (Fig. $31 \mathrm{~b}$ may help to visualize this.) One case allowed diffusion and dispersion prior to gelation, while a second case did not. After gclation, the permeability was reduced by a fac or of $30\left(F_{r r}=30\right)$ wherever gel formed. Water injectivity in layer 2 after gelation was fairly insensitive to permeability ratio for $k_{1} / k_{2}$ values between 1 and 100 . For the case with no dispersion, water injectivity after gelation increased from $61 \%$ to $62 \%$ (of original water injectivity) as the permeability ratio increased from 1 to 100 . For the case with dispersion (the dotted surve), water in jectivity after gelation increased from $64 \%$ to $68 \%$ (of original water injectivity) as the permeability ratio increased from 1 to 100 .

In two other cases in Fig. 33, no water postflush was used. After gelation, the permeability reduction in the gel bank was again equal to 30 . For the case with no dispersion (the solid curve), water injectivity after gelation increased from $11 \%$ to $18 \%$ (of original water injectivity) as the permeability ratio increased from 1 to 100 . For the case with dispersion (the dashed curve), water in jectivity af ter gelation increased from $14 \%$ to $25 \%$ (of original water in jectivity) as the permeability ratio increased from 1 to 100.

Thus, several important points should be noted from Fig. 33. First, a water postflush prior

to gelation can significantly increase injectivity in a radial geometry. Unfortunately, injectivity increases by about the same proportion in all zones. Also, diffusion and dispersion can reduce the size of a gel bank during a water pos flush. However, the bank size is reduced by about the same proportion in all zones. Thus, : water postflush usually does not help to eliminate the need for zone isolation during gel placement.

\section{Viscous Fingering: Theoretical}

I water postflush prior to gelation will tend to form viscous fingers through a viscous gelant. For a multilayer system where gelant has entered all zones, the question arises:

In which zone will viscous fingers from a water postfiush first bredk through the gelant bank? 


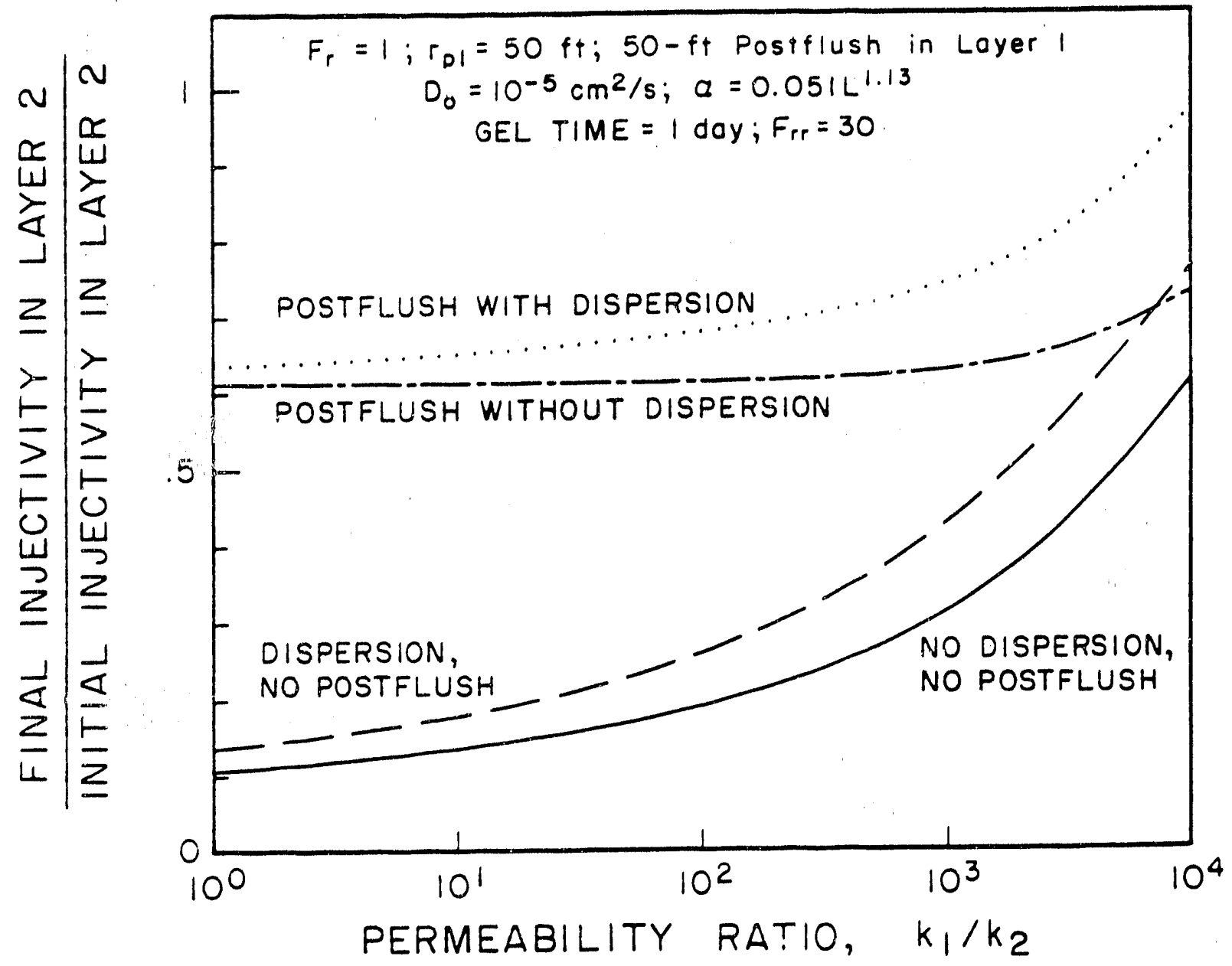

Fig. 33. Effect of a Water Postflush on Injectivity. 
When compared to the most-permeable zone, the size of the gelant bank is smaller in the lesspermeable zones, so viscous fingers have a shorter distance to travel to achieve breakthrough (see Fig. 34). However, the viscous fingers will propagate much more rapidly in the most-permeable zone. The answer to the above question will determine whether or not viscous fingering can be exploited to eliminate the need for zone isolation during gel placement.

Several researchers have quantified the growth of viscous fingers during miscible displacements. ${ }^{77,80,81}$ The analysis of Koval ${ }^{80}$ appears to be the most widely accepted. Using Koval's analysis, the region between the wellbore and the leading edge of the gelant bank can be divided into three segments (see Fig. 35). The first segment extends from the wellbore to the trailing edge of the gelant bank. Water from the postflush is the only mobile fluid in this segment. The length of this segment is $\mathrm{L}_{\mathrm{pf}} / \mathrm{EH}$ fo, linear flow and $\left[\left(\mathrm{r}_{\mathrm{pf}}{ }^{2}-\mathrm{r}_{\mathrm{o}}{ }^{2}\right) / \mathrm{EH}+\mathrm{r}_{\mathrm{o}}{ }^{2}\right]^{0.5}$ for radial flow. ${ }^{82}$ Here, $\mathrm{L}_{\mathrm{pf}}$ and $\mathrm{r}_{\mathrm{pf}}$ are the length and radius, respectively, of the water postflush if the displacement had been piston-like; $r_{o}$ is the wellbore radius; $H$ is a heterogeneity factor that ranges from 1 to 5 ; and $E$ is an effective viscosity ratio that is usually given by the quarter-power mixing rule:

$$
E=\left[0.78+0.22\left(F_{r}\right)^{0.25}\right]^{4}
$$

The second segment extends from the trailing edge of the gelant bank to the leading edge of the viscous fingers. This length corresponds to the length of the region of viscous fingers. The length of this segment for linear flow $\left(\mathrm{L}_{\mathrm{v}}\right)$ is

$$
L_{v f}=L_{p t}(E H-1 / E H)
$$

and for radial flow $\left(r_{v f}\right)$ is

$$
r_{v f}=\left[\left(r_{p f}^{2}-r_{0}^{2}\right) E H+r_{0}^{2}\right]^{p .5}-\left[\left(r_{p f}^{2}-r_{0}^{2}\right) / E H+r_{0}^{2}\right]^{0.5}
$$

The third segment extends from the leading edge of the viscous fingers to the leading edge of the gelant bank. Gelant is the only mobile fluid in this segment.

One additional piece of information is required in order to answer the question raised at the beginning of this section-that is, the effective viscosity or resistance factor of the region of viscous fingers. The maximum and minimum possible values for this resistance factor can readily be identified. The maximum value will be the resistance factor of the undiluted gelant, while the minimum value will be the resistance faztor of water (with a value equal to one). The effective viscosity for the region of viscous fingers is of ten assumed to be given by the quarter-power mixing rule $^{80-84}$ (Eq. 38). This rule is most applicable for displacement of a viscous Newtonian fluid by another Newtonian fluid. Stoneberger and Claridge ${ }^{85}$ propose Eq. 41 for unstable displacements when pseudoplastic (skear-thinning) fluids are used.

$$
E_{p}=\left[0.50+0.50\left(F_{r}\right)^{0.25}\right]^{4}
$$


In Which Layer Will Viscous Fingers

First Break Through The Gelant Bank?

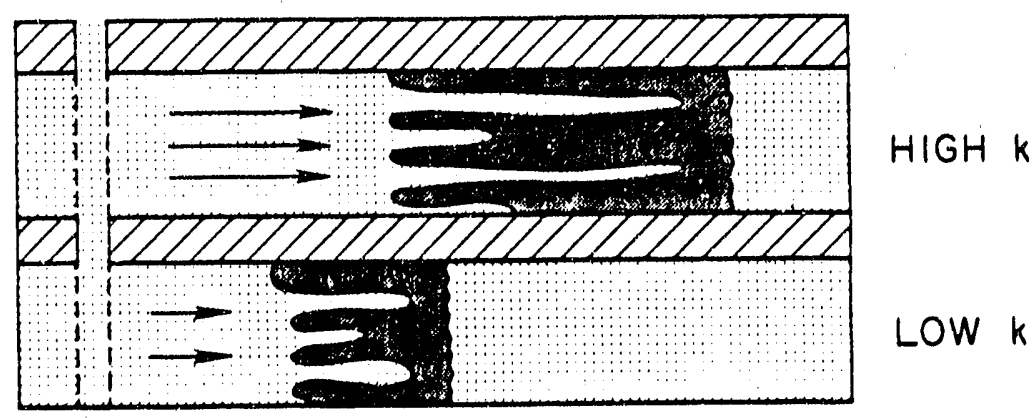

$\square$ WATER GELANT

Fig. 34. Illustration of Viscous Fingering.

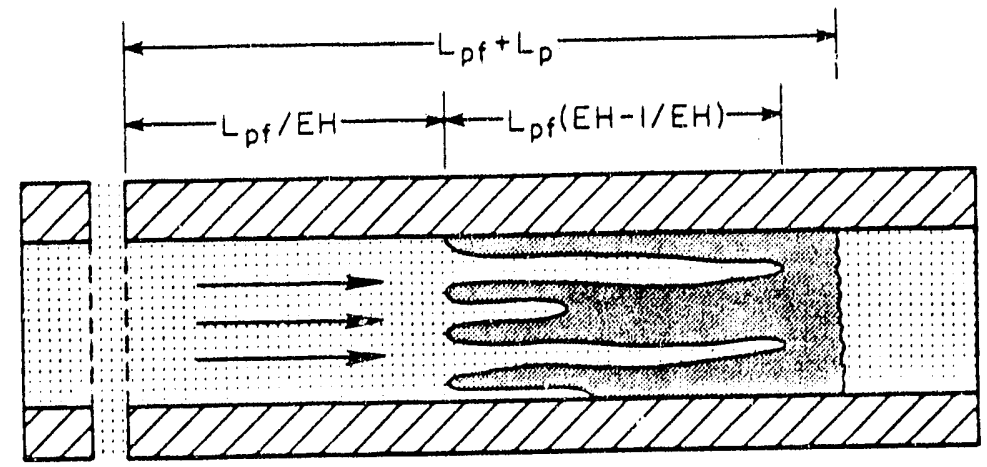

$\square$ WATER GELANT

$$
E=\left[0.78+0.22\left(F_{r}\right)^{0.25}\right]^{4}
$$

Fig. 35. Quantifying Distances During Viscous Fingering. 
Figures 36 and 37 address viscous fingering from a water postflush in radial flow. For these figures, a viscous gelant is injected to a radius of $50 \mathrm{ft}(15.24 \mathrm{~m})$ in the most-permeable layer (layer 1). During this time, the gelant penetrates some lesser distance in a given less-permeable layer (layer 2). Then, prior to gelation, watior is injected to displace the gelant. In Fig. 36 the resistance factor of the undiluted gelant $(10$. Four different values are considered for the average resistance factor of fluid in the zdrot of wiscous fingers $\left(F_{m}\right)$. The cases where $F_{m}=1$ and $F_{m}=F_{r}$ represent the extremes of possible resis anctefactors, while $F_{m}=E H$ (from the quarter-power mixing rule) provides a best guess. Using $F_{\mathrm{m}}=E_{\mathrm{p}} \mathrm{H}$ may be applicable for pseudoplastic fluids. For all four cases and for all permeability ratios, viscous fingers are predicted to break through the gelant bank in layer 1 prior to breaking through the bank in layer 2 .

Figure 37 is similar to Fig. 36 except that $F_{m}=E H$ for all cases and the resistance factor of the undiluted gelant varies from 10 to 1000 . Again, for all cases and for all permeability ratios, viscous fingers are predicted to break through the gelant bank in the most-permeable layer prior to breaking through the bank in a given less-permeable layer. However, note that for all but the most extreme permeability ratios, the fingered zone in the less-permeable layer extends most of the distance through the gelant bank.

Koval's theory has been used widely as a means of quantif ying the growth of viscous fingers. ${ }^{84}$ On the surface, our use of Koval's theory appears to provide exact predictions. However, when interpreting these predictions, one must consider the statistical nature of viscous fingering. Within a given layer, there is a random element in where fingers will form and how rapidly a given finger will grow. ${ }^{84}$ Thus, Figs. 36 and 37 must be viewed as predictions of what will occur af ter averaging many trials. On average, Fig. 37 predicts that when viscous fingers break through the bank in layer 1 , the fingers will have traversed typically $60 \%$ to $100 \%$ of the bank in layer 2 . Alternatively, Fig. 37 predicts that viscous fingers will break through in layer 1 somewhat more of ten than in layer 2.

Figures 38 and 39 provide predictions for viscous fingering from a water post-flush in linear flow. The conditions represented in this figure are analogous to those used in generating Figs. 36 and 37. Figure 39 predicts that, on average, viscous fingers will break through the gelant bank in a given less-permeable layer at about the same time as in the most-permeable layer.

In Figs. 36 and 39, the Koval heterogeneity factor $(\mathrm{H})$ was assumed to have a value of one in each layer. We have examined the effect of $\mathrm{H}$ on the predictions and have found that the results are fairly insensitive to the choice of heterogeneity factor.

The reader should note that Figs. 24, 28, 29, 32, 33, 37, and 39 can be applied to reservoirs with many layers. For example, consider a reservoir that has four noncommunicating layers, with permeabilities of $10,000 \mathrm{md}, 1000 \mathrm{md}, 100 \mathrm{md}$, and $10 \mathrm{md}$, respectively. Assume that all layers have the same porosity and that flow is radial. If a 10 -cp fluid were injected to displace 1 -cp water, then when the viscous fluid reached a radius of $50 \mathrm{ft}$ from the wellbore in the 10,000-md layer, the fluid would have reached radii of $17.9 \mathrm{fr}, 6.7 \mathrm{ft}$, and $2.6 \mathrm{ft}$ in the 1000-md, $10 \mathrm{~m}$-md, and 10-md layers, respectively. If a water postflush was subsequentiy injected until viscous fingers broke through the viscous bank in the 10,000-md layer, then Fig. 37 can be used to find how far viscous fingers had propagated in the other layers. At breakthrough in the 10,000-md layer, the fingers are predicted to traverse $85 \%, 70 \%$, and $53 \%$ of the viscous banks in the $1000-\mathrm{md}, 100-\mathrm{md}$, and 10-md layers, respectively. 


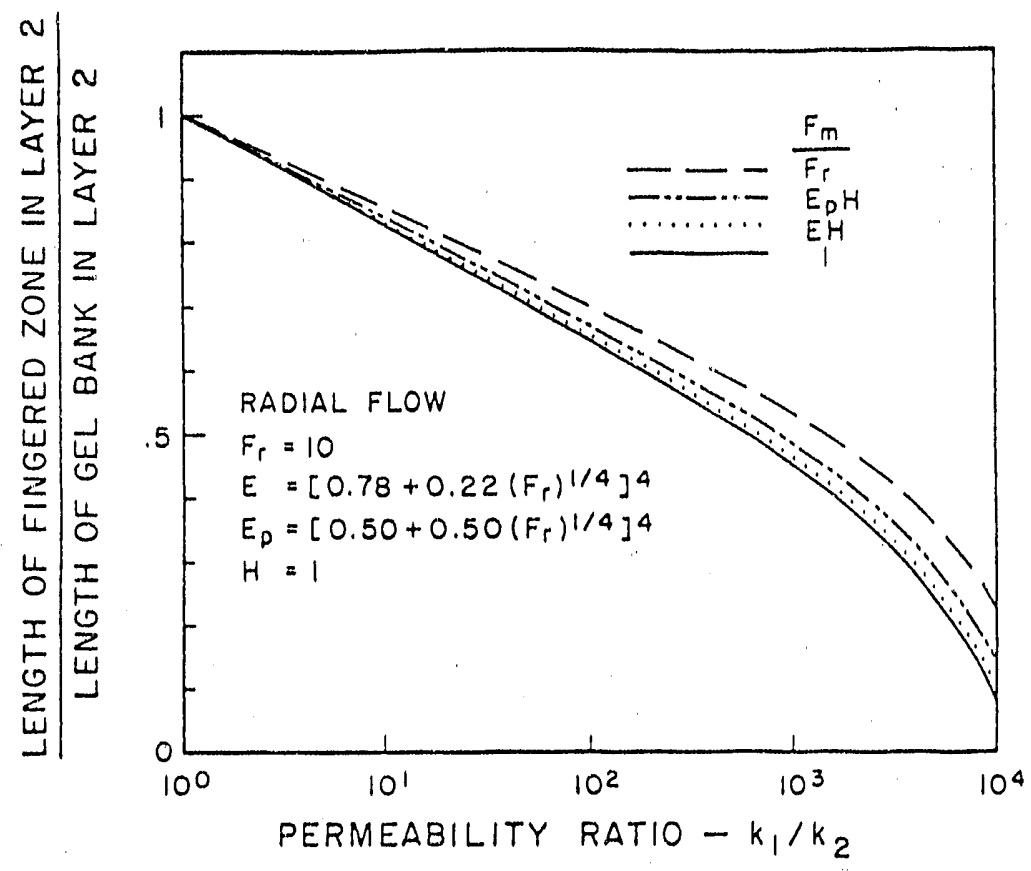

Fig. 36. Sensitivity of Finger Breakthrough to Assumed Resistance Factor in the Fingered Zone: Radial Flow. Length of Fingered Zone in Layer 2 when Fingers Break Through in Layer 1.

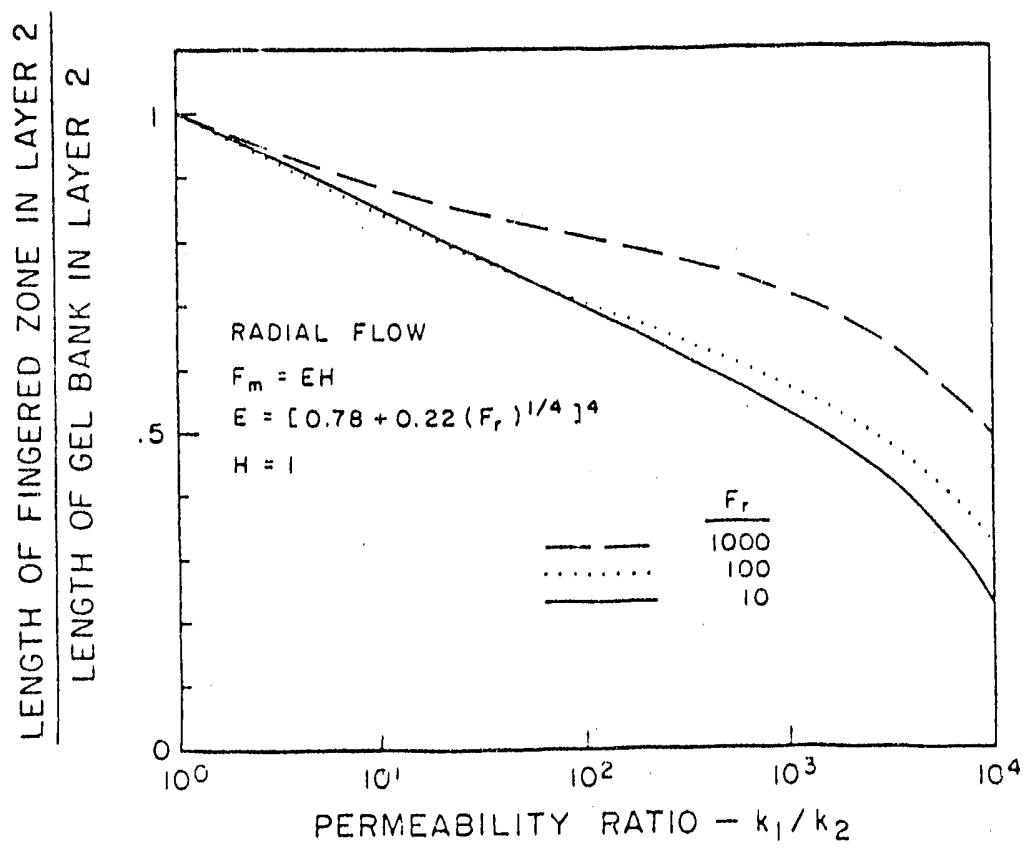

Fig. 37. Sensitivity of Finger Breakthrough to Resistance Factor of the Viscous Fluid: Radial Flow. Length of Fingered Zone in Layer 2 when Fingers Break Through in Layer 1. 


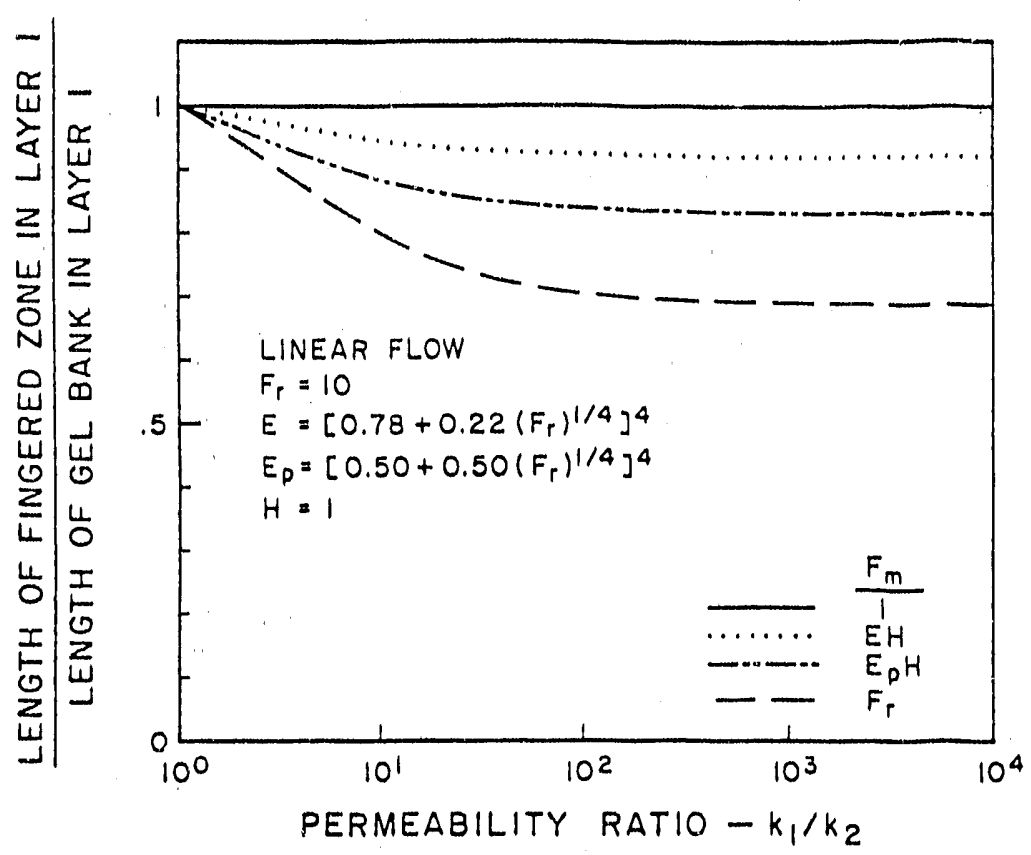

Fig. 38. Sensitivity of Finger Breakthrough to Assumed Resistance Factor in the Fingered Zone: Linear Flow. Length of Fingered Zone in Layer 1 when Fingers Break Through in Layer 2.

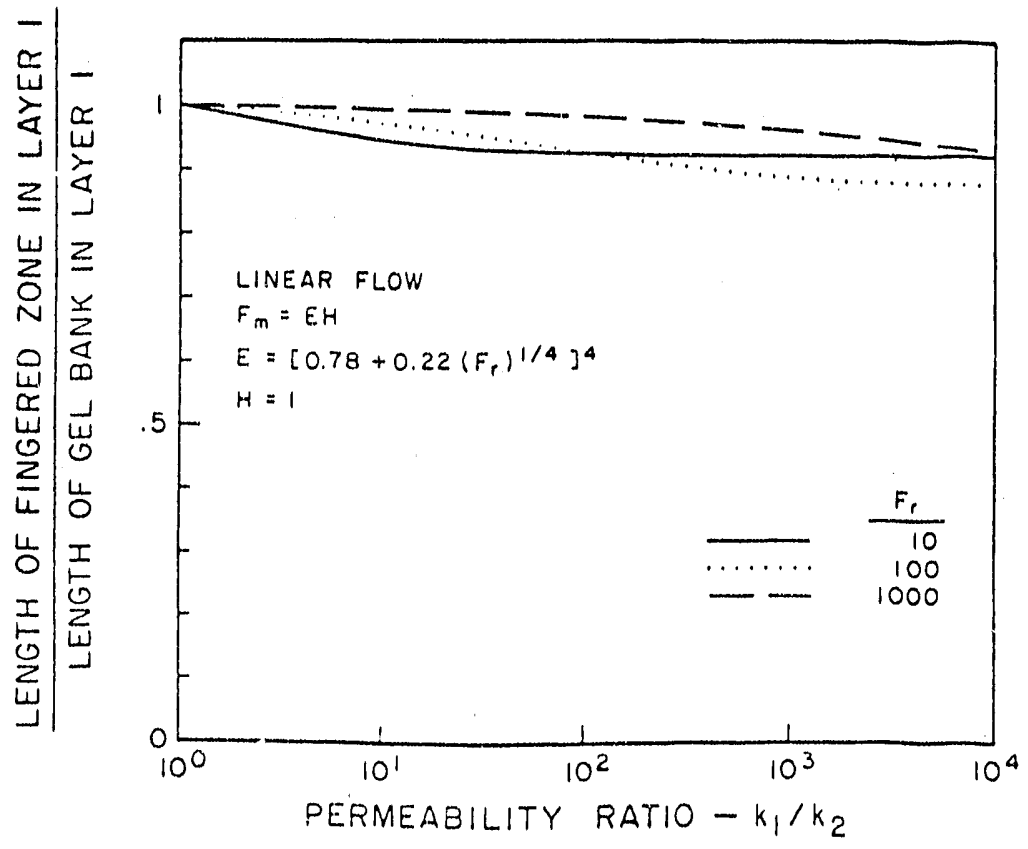

Fig. 39. Sensitivity of Finger Breakthrough to Resistance Factor of the Viscous Fluid: Linear Flow. Leng th of Fingered Zone in Layer 1 when Fingers Break Through in Layer 2. 


\section{Viscous Fingering: Experimental Verification}

To test the radial-flow predictions, sequential banks of water, viscous fluid, and water were injected into parallel beadpacks. Each beadpack was configured as one-quarter of a five-spot pattern, with one injector and one producer in diagonally opposite corners (see Fig. 40). The dimensions of the packs were $56 \mathrm{~cm} \times 56 \mathrm{~cm} \times 1.2 \mathrm{~cm}$. The less-permeable pack contained nominally $150-\mu \mathrm{m}$ glass beads and had a permeability of 13.6 darcies $\left(13.4 \mu \mathrm{m}^{2}\right)$. The more-permeable pack contained nominally $500-\mu \mathrm{m}$ glass beads and exhibited a permeability of 174 darcies $\left(172 \mu \mathrm{m}^{2}\right)$. The porosity of both packs was 0.38 , and both packs were initially completely saturated with water. The two packs were flooded in parallel-experiencing the same pressure drop and injection fluid at any given time. The total fluid injection rate (pack 1 plus pack 2) was maintained constant at $1440 \mathrm{ml} / \mathrm{hr}$.

A viscous water-miscible fluid was injected to reach a radius $\left(r_{p 1}\right)$ of approximately $35 \mathrm{~cm}$ in the most-permeable beadpack. The radius to which the fluid penetrated in the less-permeable pack $\left(r_{\mathrm{p} 2}\right)$ was then noted (see Table 25). Fluorescein dye was includied with the viscous bank to allow visualization of its boundaries. Three viscous fluids were used: (1) a mixture of $85 \%$ ethanediol and $15 \%$ water, (2) 2000 -ppm xanthan in water, and (3) 2000 -ppm HPAM in aqueous $0.5 \% \mathrm{KCl}$. The ethanediol mixture was Newtonian with a viscosity of $11 \mathrm{cp}$. The xanthan solution was shearthinning, exhibiting a power-law exponent of 0.36 and providing a viscosity of $99 \mathrm{cp}$ at $11 \mathrm{~s}^{-1}$. The HPAM solution was also shear-thinning, exhibiting a power-law exponent of 0.69 and providing a viscosity of $30 \mathrm{cp}$ at $11 \mathrm{~s}^{-1}$. Experiments confirmed that polymer adsorption in the beadpacks was negligible. All experiments were conducted at room temperature.

Consistent with the predictions of Refs. 24 and 25 , all three viscous agents penetrated to a significant degree into the less-permeable pack. The ratio, $r_{p 2} / r_{p 1}$, was greater than the square root of the permeability ratio, $k_{2} / k_{j}$, in all three cases.

After placement of the viscous banks, water (without fluorescein) vas injected until viscous fingers broke through one of the two viscous banks. Injection was then stopped. Note was made of the following:

1. The bank that was first breached by viscous fingers,

2. The final outer radius of the viscous bank in each pack $\left(r_{f o 1} \& r_{f o 2}\right)$,

3. The final inner radius of the viscous bank in each pack $\left(r_{\tilde{\pi} 1} \& r_{\tilde{1} 2}\right)$,

4. The number of fingers in each bank,

5. The length of the longest finger in each bank $\left(r_{v 11} \& r_{v 22}\right)$,

6. The length of the songest finger divided by the final length of the viscous bank, and

7. The ratio, $r_{f o} / r_{\tilde{f}}$, for each pack ( $w$ hich, in theory, should approximately equal EH).

The fingering patterns did not show signs of repeating the same flow paths from run to run-indicating good homogeneity in the beadpacks. For each viscous fluid, the parallel displacement experiments were repeated 9 to 12 times. Figure 40 illustrates the important measurements that were made during each experiment. Averages and standard deviation values for the various measurements are listed in Table 25. A listing of results for individual experiments can be found in Appendix E. 


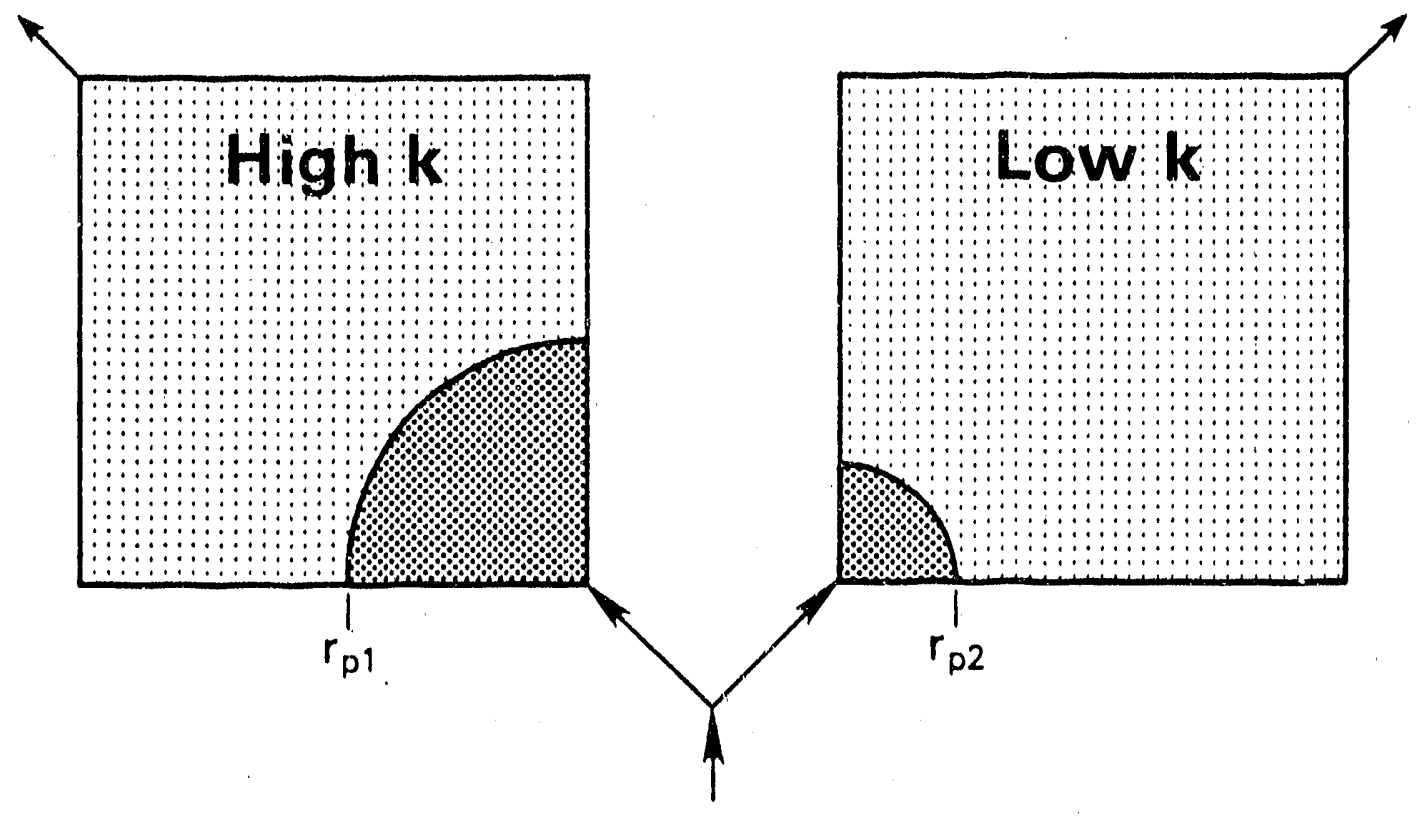

WATER VISCOUS FLUID

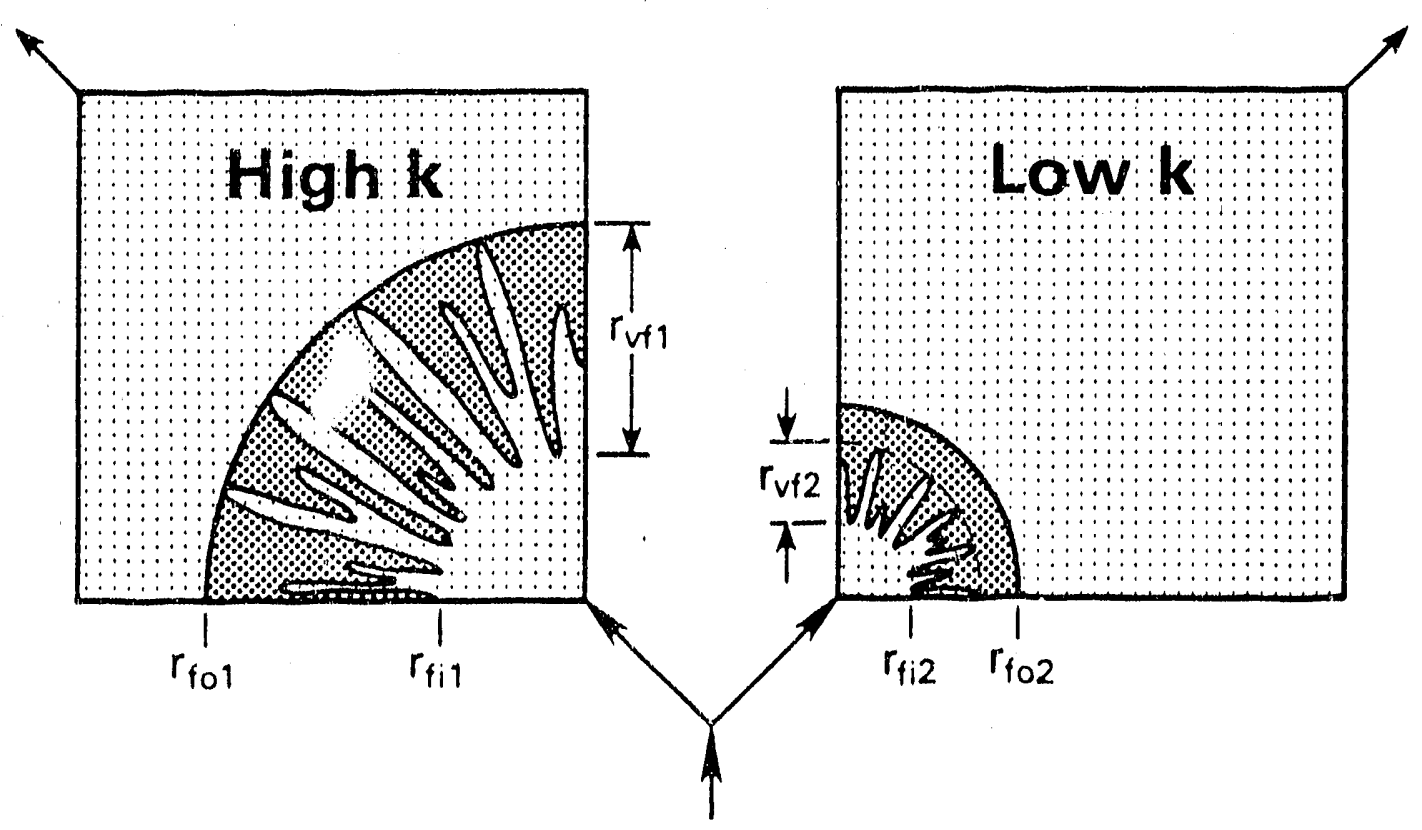

Fig. 40. Important Measurements during Viscous Fingering Experiments. 
TABLE 25

Summary of Viscous-Fingering Results

\begin{tabular}{|c|c|c|c|c|c|c|}
\hline Viscous Fluid: & Ethaned & Liol & 2000-00m & Santhan & 2000-pom & HPAM \\
\hline Number of replicates: & 10 & & 12 & & 9 & \\
\hline & $\begin{array}{l}\text { High k } \\
\text { Pack } \\
\end{array}$ & $\begin{array}{l}\text { Low k } \\
\text { Pack }\end{array}$ & $\begin{array}{l}\text { High k } \\
\text { Pack } \\
\end{array}$ & $\begin{array}{l}\text { Low k } \\
\text { Pack } \\
\end{array}$ & $\begin{array}{l}\text { High k } \\
\text { Pack } \\
\end{array}$ & $\begin{array}{l}\text { Low k } \\
\text { Pack }\end{array}$ \\
\hline $\begin{array}{l}\text { Initial radius of } \\
\text { viscous fluid }(\mathbf{c m}) \text { : }\end{array}$ & $35.2 \pm 1.3$ & $14.1 \pm 1.9$ & $35.8 \pm 0.2$ & $11.6 \pm 0.7$ & $33.7 \pm 1.2$ & $15.1 \pm 1.9$ \\
\hline $\begin{array}{l}\text { Occasions viscous bank } \\
\text { is breached first: }\end{array}$ & 7 & 3 & 9 & 3 & 8 & 1 \\
\hline $\begin{array}{l}\text { Final outer radius of } \\
\text { viscous fluid }(\mathrm{cm}) \text { : }\end{array}$ & $41.9 \pm 2.9$ & $15.8 \pm 1.8$ & $38.8 \pm 0.9$ & $12.2 \pm 0.8$ & $39.7 \pm 1.3$ & $15.8 \pm 1.9$ \\
\hline $\begin{array}{l}\text { Final inner radius of } \\
\text { viscous fluid }(\mathrm{cm}) \text { : }\end{array}$ & $19.9 \pm 4.4$ & $9.6 \pm 2.1$ & $8.0 \pm 2.2$ & $4.3 \pm 0.5$ & $12.0 \pm 1.5$ & $4.8 \pm 0.8$ \\
\hline Number of fingers: & $5 \pm 1$ & $18 \pm 7$ & $12 \pm 3$ & $15 \pm 5$ & $7 \pm 2$ & $6 \pm 2$ \\
\hline $\begin{array}{l}\text { Length of longest } \\
\text { viscous fingor }(\mathrm{cm}) \text { : }\end{array}$ & $20.4 \pm 2.7$ & $4.5 \pm 2.6$ & $30.2 \pm 2.1$ & $6.9 \pm 1.2$ & $27.5 \pm 1.1$ & $6.8 \pm 2.9$ \\
\hline $\begin{array}{l}\text { Length of fingered zone } \\
\text { relative to length of } \\
\text { viscous bank: }\end{array}$ & $0.93 \pm 0.10$ & $0.71 \pm 0.22$ & $0.98 \pm 0.03$ & $0.86 \pm 0.10$ & $0.99 \pm 0.03$ & $0.62 \pm 0.16$ \\
\hline E from fingering data: & $2.1 \pm 0.3$ & $1.6 \pm 0.5$ & $5.2 \pm 1.7$ & $2.6 \pm 0.4$ & $3.3 \pm 0.4$ & $2.5 \pm 0.9$ \\
\hline E from Eq. 38: & 1.9 & 1.9 & 2.8 & 2.7 & 2.4 & 2.3 \\
\hline E from Eq. 41 : & 4.0 & 4.0 & 7.7 & 7.1 & 5.8 & 5.6 \\
\hline
\end{tabular}

For all three viscous fluids, viscous fingers broke through the bank in the high-permeability pack more of ten than in the low-permeability pack. However, regardless of which pack first experienced breakthrough, fingers had traversed most of the viscous bank in the other beadpack. These findings are qualitatively in agreement with the theory, and they have important implications with respect to in-situ dilution and mixing of gelling agents. The results suggest that viscous fingers will facilitate in-situ mixing of fluids to about the same extent in low-permeability zones as in highpermeability zones. This is beneficial if uniform mixing of fluids is desired. However, the results cast doubt on the utility of schemes that rely on nonuniform mixing to optimize gel placement. For example, fingers from a water postflush are not expected to eliminate the need for zone isolation during gel placement (i.e., by breaking through a gelant bank in a low-permeability layer before breaking through in a high-permeability layer). Also, one can not rely on viscous fingering to promote or inhibit gelation in one zone to a greater extent than in another zone.

The above observations also have an important implication in traditional polymer floods where crossflow can occur between layers. Reserchers ${ }^{86-88}$ have suggested that a small bank of a viscous fluid can cause surprisingly high leveis of incremental oil recovery if crossflow can occur in a stratified reservoir. They argue that the viscous bank in a high-permeability layer can outrun the bank in less-permeable layers. Then, water behind the viscous bank in the high-permeability layer is forced to cross flow into and displace oil from the less-permeable layers. 
The observations of viscous fingering reported here introduce questions about the validity of the above displacement mechanism. The above mechanism requires that significant volumes of water from the postflush must cross flow from the high-permeability layer into the low-permeability layer. By implication, this requires that viscous fingers must somehow break through the viscous bank in the low permeability layer substantially before breakthrough in the high-permeability layer. Our findings raise doubt that this will happen. More work is needed to resolve this issue.

The factor, E, provides one means to compare theoretical predictions with experimental results. As mentioned earlier, the outer radius of the fingered zone is $\left[\left(r_{p f}{ }^{2}-r_{0}{ }^{2}\right) E H+r_{o}{ }^{2}\right]^{0.5}$, while the inner radius of the fingered zone is $\left[\left(r_{\mathrm{pt}}{ }^{2}-\mathrm{r}_{0}{ }^{2}\right) / E H+\mathrm{r}_{\mathrm{o}}{ }^{2}\right]^{0.5}$. If the heterogeneity factor $(\mathrm{H})$ equals one (which should be the case for our homogeneous beadpacks) and if the wellbore radius $\left(r_{0}\right)$ is negligible, $E$ may be estimated from experimental data. In particular, $E$ is approximately equal to the outer radius of the fingered zone divided by the inner radius of the fingered zone $\left(r_{\mathrm{fo}} / r_{\mathrm{ff}}\right)$. The bottom of Table 25 lists values for $E$ that were determined in this way from the experimental data. For comparison, Table 25 also lists values for $E$ that were calculated using Eqs. 38 and 41 . (In the high- and low-permeability packs, the geometrically averaged values for $F_{r}$ that were used in Eqs. 38 and 41 were (1) 11 and 11 , respectively, for ethanediol; (2) 30 and 26 , respectively, for the xanthan solution; and (3) 20 and 19, respectively, for the HPAM solution.) For all but one of the pack-fluid combinations, Eq. 38 provided a reasonably close match to the experimentally determined value for E. The exception was the xanthan-high-permeability-pack data. Eq. 41 overestimated the experimental value for $\mathrm{E}$ in all cases.

Note in Table 25 that the final outer radius of the viscous fluid is least for the 2000 -ppm xanthan solution and is greatest for the ethanediol mixture. This is true for both the high- and the low-permeability packs. This indicates that viscous fingering is most severe for the xanthan solution and least severe for the ethanediol. Two factors may be responsible for this ordering. First, fingering becomes more severe with increased mobility contrast. (The xanthan solution is the most viscous, while ethanediol is the least viscous of the three fluids.) Second, fingering is reported to be more severe for shear-thinning fluids than for Newtonian fluids. ${ }^{85,89}$ (The xanthan solution is the most pseudoplastic of the fluids, while ethanediol is Newtonian.)

\section{Conclusions}

1. For near-wellbore gel treatments in unfractured in jection wells, diffusion and/or dispersion usually will not eliminate the need for zone isolation during gel placement.

2. During gel placement in parallel laboratory corefloods, diffusion and dispersion can mislead one to conclude that zone isolation is not needed during gel placement in field projects.

3. For near-wellbore gel treatments in unfractured injection wells, a water postflush usually will not eliminate the need for zone isolation during gel placement.

4. If a viscous fluid is injected into a radial, multilayer system and then followed by a water postflush, both theory and experiments indicate that viscous fingers usually will break through the viscous bank in the most-permeable layer first. However, at the time of this breakthrough, the tingers will have traversed most of the viscous bank in a less-permeable layer. 


\section{AN EXAMINATION OF FLOW-PROFILE CHANGES FOR FIELD APPLICATIONS OF GEL TREATMENTS IN INJECTION WELLS}

In several reports, $24,25,26$ theoretical analyses are presented that examine how flow profiles in injection wells are modified when zones are not isolated during gel placement. An important prediction of these investigations is that injection profiles usually are not expected to improve significantly in unfractured wells if gels are placed without using zone isolation.

The question arises, "Does field experience with gel treatments in injection wells confirm or contradict the conclusions of these papers?"

Before answering this question, several points should be made to narrow the scope of the issue to be addressed here. First, we recognize that changing the flow profile in an injection well will not insure that sweep efficiency will be improved in a reservoir. Particularly if crossflow can occur between strata, changes in injection profiles are irrelevant beyond the outer radii of the gel banks. Also, flow behind pipe can negate any significance of a measured injection profile.

Second, changes in oil production rates or in water cuts in nearby production wells are not necessarily relevant to the question raised here. Responses in production wells are strongly influenced by well workovers, pumping rates in produrtion wells, and changes in injection rates in all nearby wells-not just by changes in injection profiles in a given well.

Third, we recognize that flow profiles in a vertically fractured well can be meaningless. The high conductivity of the fracture can readily allow fluids to move vertically (within the fracture) after they leave the wellbore. Thus, this analysis is not intended to argue that an observed change of an injection profile in a fractured well has some meaning. Instead, the objective here is to establish whether or not injection profiles in unfractured wells are improved if gels are placed without zone isolation.

Certain limitations must be understood when trying to establish the flow profile in an injection well. First, the tools and methods that are used to measure flow rofiles (spinner surveys and radioactive-tracer flow logs) have limitations. Typically, these tools measure the flow past a given point to within plus or minus five to ten percent of the total flow. ${ }^{90}$ Thus, changes that amount to less than $\pm 5-10 \%$ of total flow are not usually significant. Second, the methods have limitations on how precisely they can pinpoint where the fluid leaves the wellbore. Furthermore, for positions that are separated vertically by only a few feet, one must consider the likelihood of flow behind pipe and vertical crossflow. Unless unusual precautions are taken, different elevations of a given profile should not be considered distinct unless they are separated by a reasonable distance (several feet). 


\section{Analysis Example}

When interpreting injection profile changes, some reports apparently failed to consider some of the above limitations. Results reported by Chang et al. ${ }^{65}$ will be used to illustrate this. Fig. $4 i$ is taken from Fig. 15 of Ref. 65. This figure shows the results from six injection profiles that were measured in one well. The first profile was obtained during water injection prior to the gel treatment. The four subsequent profiles were taken during injection of a xanthan gelling agent. The final profile was obtained during water injection after the gel treatment. Based on this figure, Chang et al. concluded:

1) The fraction of fuid entering the most-permeable zone increased when a xanthan formulation was injected instead of water.

2) The final injection profile during water injection after gel placement was more favorable than the injection profile during water injection prior to the gel treatment.

Chang et al. do not provide an analysis of the profiles to support their conclusions. Therefore, an analysis will be provided here.

For all profiles in Fig. 41 , most fluid enters the 5-10 ft interval that is centered at a depth of $1578 \mathrm{ft}$. This interval should be considered as a single zone. The tool limitations mentioned previously provide one reason for doing this. A second reason is that all strata in the bottom 5-10 feet are probably connected (either by flow behind pipe or by vertical crossflow). Attempts to distinguish details of the flow profile in the lowest zone would be of questionable value.

Figure 42 identifies the percentages of fluid that enter the different zones for the six injection profiles. During water injection prior to the gel treatment, $63 \%$ of the fluid entered the bottom zone. During in jection of the xanthan gel formulation, the percentages of fluid that entered the bottom zone varied between $53 \%$ and $70 \%$ in no discernable pattern. During this period the average percentage (and standard deviation) was $61 \%( \pm 7 \%)$. During water injection after placement of the gel, the percentage of fluid entering the bottom zone was $60 \%$. Thus, the percentage of fluid entering the bottom zone did not change significantly either during xanthan injection or as a final resulic of the gel treatment.

Of the remaining intervals in well WI-A, there are no changes in the flow profiles that look definitive. One might argue that a zone opened at 1525-1530 ft in the SWAT $A$ and post-treatment profiles. However, aside from fracturing the well in that zone, what argument could be used to. justify this? How could the permeability of this zone suddenly go from zero to some value that would account for 10 percent of the total flow? Similarly, it appears that 10 percent of the fluid left the wellbore at $1538 \mathrm{ft}$ in the pre-treatment profile; then this zone was somehow plugged during the next 3-4 profiles, and somehow became unplugged during the last 1-2 profiles. How could this have happened? The answers to these questions probably have more to do with linitations of the method of measuring profiles thar it does with real profile changes.

Thus, an analysis of Chang's profiles reveals that (within the limitations of the measurements) no significant profile changes occurred either during injection of the xanthan gelant or as a final result of the gel treatment. 
SELECTIVELY EMPLACED BIOPOLYMER GEL MODIFIED INJECTION PROFILE

FIELL A, WELL WI-A

Y FLOW

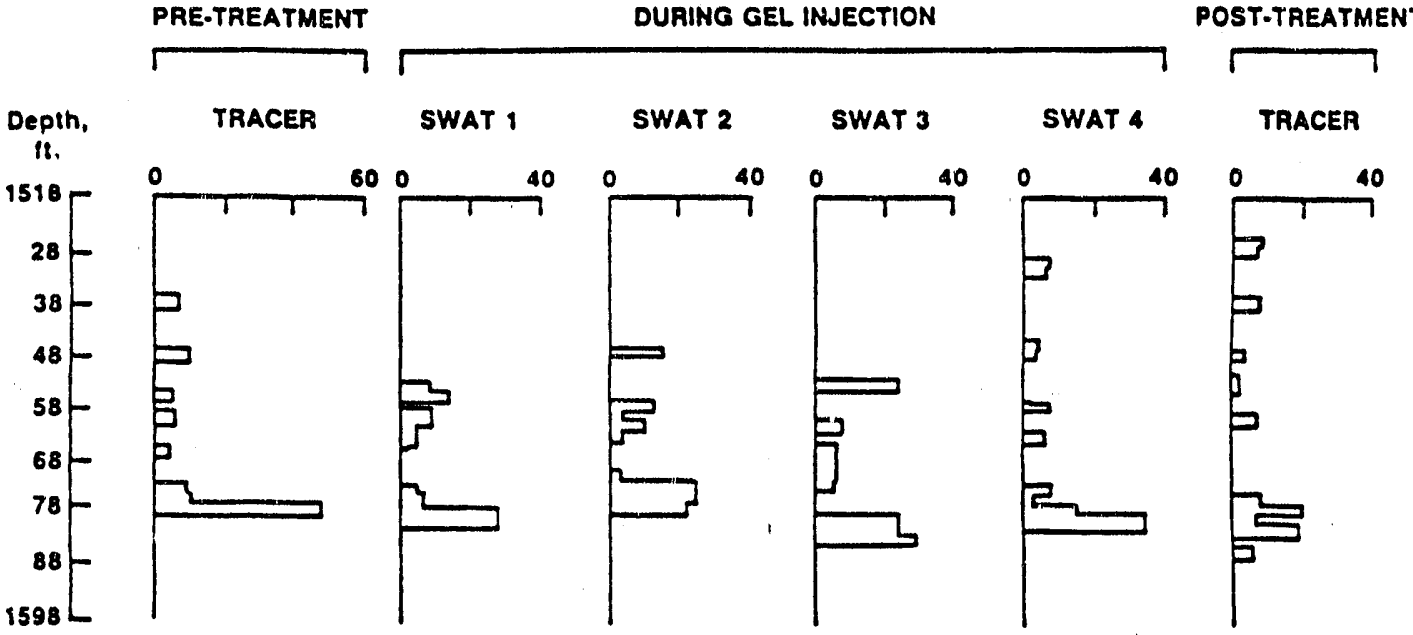

Fig. 41. Injection Profiles from Chang et al. ${ }^{65}$

\section{SELECTIVELY EMPLACED BIOPOLYMER GEL MODIFIED INJECTION PROFILE}

FIELD A, WELL WI-A

\% FLOW

PRE-TREATMENT

DURING GEL INJECTION

POST-TAEATMENT
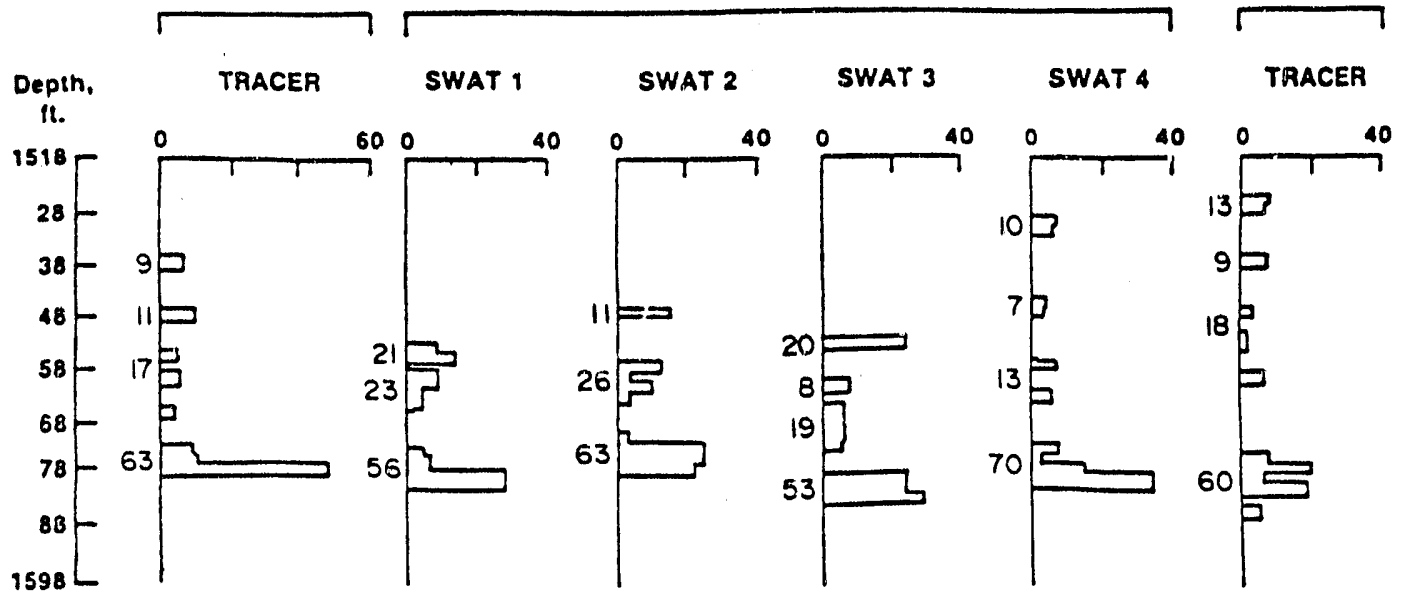

Fig. 42. Injection Profiles with Flow Percentages. 


\section{Analysis Summary for 43 Pairs of Injection Proflies}

Table 26 provides a summary of similar analyses of 43 pairs of injection profiles that were reported in the literature. ${ }^{31,33,46,64,91-98}$ For each pair, one profile was obtained before the gel treatment, and another profile was obtained after the gel treatment. Zones were not isolated during gel placement. For each entry in Table 26, the following questions are addressed:

1. What was the gelant?

2. Was the profile changed significantly as a result of the gel treatment?

3. Was the profile improved as a result of the gel treatment?

4. Was the injector fractured prior to the gel treatment?

5. What was the lithology of the reservoir?

6. Was injectivity (BPD/psi) reported both before and after the gel treatment?

7. What was the literature source? (Specific figures used from each source are included in the reference column of Table 26 in parentheses.)

A question mark (?) indicates that no information was provided concerning the listed item. Entries that are grouped together in Table 26 refer to wells that were treated within the same field.

About 30 percent of the entries in Table 26 demonstrate a clear improvement in the injection profile resulting from a gel treatment. About 40 percent show no significant improvement. In the remaining cases, improvements in injection profiles are slight or debatable.

Unfortunately, the literature does not provide enough information to surrelate profile improvement with whether or not a well is fractured. In $21 \%$ of the cases, the wells were reported to be fractured. In the remaining $79 \%$ of the cases, the literature sources did not state whether or not the wells were fractured. Because of the widespread use of fracturing to stimulate walls, one can not simply assume that all of these wells were not fractured. Between 1948 and 1968, more than 500,000 wells were intentionally fractured. ${ }^{99}$ Many other wells have been fractured unintentionally during waterflood operations. Without knowing whether or not a given well was fractured, we can not establish that injection profiles can be improved in unf ractured injection wells without rone isolation.

Assessing whether or not zones can or should be isolated during gel placement is a critical issue that must be addressed. Gel treatments account for approximately $20 \%$ of all EOR projects in the United States. In spite of the large number of projects, less than two percent of the total EOR oil production rate can be attributed to these gel treatments. 5,6 This fact is consistent with a widespread view in the industry that the success of gel treatments in injection wells has been very sporadis. A study conducted in 1985 revealed that less than $45 \%$ of near-wellbore gei treatments were successful. ${ }^{7}$ In the vast majority of gel applications during the 1980's, zones were not isolated during ge: placement. This practice could be partly responsible for the sporadic success rate for gel treatments. 
TABLE 26

Published Injection Profiles: Before versus After Gel Treatments

\begin{tabular}{|c|c|c|c|c|c|c|}
\hline Gelant & $\begin{array}{l}\text { Profile } \\
\text { Changed? }\end{array}$ & $\begin{array}{l}\text { Profile } \\
\text { Improved? }\end{array}$ & $\begin{array}{l}\text { Injector } \\
\text { Fractured? }\end{array}$ & Lithology & $\begin{array}{l}\text { Before/After } \\
\text { Injectivity } \\
\text { Given? } \\
\end{array}$ & Reference \\
\hline Cr-xanthan & no & no & $?$ & sandstone & no & 65 (Fig. 15) \\
\hline $\begin{array}{l}\text { Cr-xanthan } \\
\text { Cr-xanthan } \\
\text { Cr-xanthan } \\
\text { Cr-xanthan }\end{array}$ & $\begin{array}{l}\text { no } \\
\text { slightly } \\
\text { slightly } \\
\text { yes }\end{array}$ & $\begin{array}{l}\text { no } \\
\text { slightly } \\
\text { slightly } \\
\text { yes }\end{array}$ & $\begin{array}{l}? \\
? \\
? \\
?\end{array}$ & $\begin{array}{l}\text { sandstone } \\
\text { sandstone } \\
\text { sandstone } \\
\text { sandstone }\end{array}$ & $\begin{array}{l}\text { no } \\
\text { no } \\
\text { no } \\
\text { no }\end{array}$ & $\begin{array}{l}91 \text { (Fig. 4) } \\
91 \text { " " } \\
91 \text { " " } \\
91 \text { " " }\end{array}$ \\
\hline $\begin{array}{l}\mathrm{Cr} \text {-xanthan } \\
\mathrm{Cr} \text {-xanthan } \\
\mathrm{Cr} \text {-xanthan } \\
\mathrm{Cr} \text {-xanthan }\end{array}$ & $\begin{array}{l}\text { slightly } \\
\text { yes } \\
\text { yes } \\
\text { yes }\end{array}$ & $\begin{array}{l}\text { debatable } \\
\text { yes } \\
\text { no } \\
\text { no }\end{array}$ & $\begin{array}{l}? \\
? \\
? \\
?\end{array}$ & $\begin{array}{l}\text { sandstone } \\
\text { sandstone } \\
\text { sandstone } \\
\text { sandstone }\end{array}$ & $\begin{array}{l}\text { no } \\
\text { no } \\
\text { no } \\
\text { no }\end{array}$ & $\begin{array}{l}91 \text { (Fig. 7) } \\
91 \text { " " } \\
91 \text { " " } \\
91 \text { " }\end{array}$ \\
\hline $\begin{array}{l}\text { Cr-xanthan } \\
C r-x a n t h a n \\
\text { Cr-xanthar } \\
C r-x a n t h a n\end{array}$ & $\begin{array}{l}\text { slightly } \\
\text { slightly } \\
\text { slightly } \\
\text { no }\end{array}$ & $\begin{array}{l}\text { debatable } \\
\text { debatable } \\
\text { slightly } \\
\text { no }\end{array}$ & $\begin{array}{l}? \\
? \\
? \\
?\end{array}$ & $\begin{array}{l}\text { sandstone } \\
\text { sandstone } \\
\text { sandstone } \\
\text { sandstone }\end{array}$ & $\begin{array}{l}\text { yes } \\
\text { yes } \\
\text { yes } \\
\text { yes }\end{array}$ & $\begin{array}{l}33 \text { (Fig. } 7 \text { ) } \\
33 \text { " } \\
33 \text { (Fig. } 8 \text { ) } \\
33 \text { " }\end{array}$ \\
\hline $\begin{array}{l}\text { Cr-xanthan } \\
\text { Cr-xanthan } \\
\text { Cr-xanthan }\end{array}$ & $\begin{array}{l}\text { yes } \\
\text { yes } \\
\text { yes }\end{array}$ & $\begin{array}{l}\text { debatable } \\
\text { debatable } \\
\text { no }\end{array}$ & $\begin{array}{l}? \\
? \\
?\end{array}$ & $\begin{array}{l}\text { sandstone } \\
\text { sandstone } \\
\text { sandstone }\end{array}$ & $\begin{array}{l}\text { no } \\
\text { no } \\
\text { no }\end{array}$ & $\begin{array}{l}33 \text { (Fig. 10) } \\
33 " . " \\
33 " .\end{array}$ \\
\hline $\begin{array}{l}\text { Cr-xanthan } \\
\text { Cr-xanthan }\end{array}$ & $\begin{array}{l}\text { no } \\
\text { debatable }\end{array}$ & $\begin{array}{c}\text { no } \\
\text { debatable }\end{array}$ & $?$ & $\begin{array}{l}\text { sandstone } \\
\text { sandstone }\end{array}$ & $\begin{array}{l}\text { no } \\
\text { no }\end{array}$ & 33 (Fig. 13) \\
\hline $\begin{array}{l}\text { Cr-xanthan } \\
\text { Cr-xanthan }\end{array}$ & $\begin{array}{l}\text { yes } \\
\text { yes }\end{array}$ & $\begin{array}{l}\text { yes } \\
\text { yes }\end{array}$ & $\begin{array}{l}? \\
?\end{array}$ & $\begin{array}{l}\text { sandstone } \\
\text { sandstone }\end{array}$ & $\begin{array}{l}\text { no } \\
\text { no }\end{array}$ & $\begin{array}{l}33 \text { (Fig. 15) } \\
33\end{array}$ \\
\hline $\begin{array}{l}\text { Cr-xanthan } \\
\text { Cr-xanthan } \\
\text { Cr-xanthan } \\
\text { Cr-xanthan }\end{array}$ & $\begin{array}{c}\text { no } \\
\text { yes } \\
\text { yes } \\
\text { slightly }\end{array}$ & $\begin{array}{c}\text { no } \\
\text { no } \\
\text { yes } \\
\text { slightly }\end{array}$ & $\begin{array}{l}? \\
? \\
? \\
?\end{array}$ & $\begin{array}{l}\text { sandstone } \\
\text { sandstone } \\
\text { sandstone } \\
\text { sandstone }\end{array}$ & $\begin{array}{l}\text { no } \\
\text { no } \\
\text { no } \\
\text { no }\end{array}$ & $\begin{array}{l}92 \text { (Fig. 6) } \\
92 \text { " " " } \\
92 . \\
92 . "\end{array}$ \\
\hline Cr-xanthan & yes & no & yes & sandstone & yes & 92 (Fig. 7) \\
\hline $\begin{array}{l}\text { resorcinol- } \\
\text { formaldehyde }\end{array}$ & no & no & $?$ & sandstone & yes & 31 (Fig. 11) \\
\hline $\begin{array}{l}\text { phenol- } \\
\text { formaldehyde }\end{array}$ & yes & yes & $?$ & carbonate & no & 46 (Fig. 8) \\
\hline $\begin{array}{l}\text { alcaligenes } \\
\text { alcaligenes } \\
\text { alcaligenes } \\
\text { alcaligenes }\end{array}$ & $\begin{array}{c}\text { no } \\
\text { no } \\
\text { no } \\
\text { debatable }\end{array}$ & $\begin{array}{c}\text { no } \\
\text { no } \\
\text { no } \\
\text { debatable }\end{array}$ & $\begin{array}{l}\text { yes } \\
\text { yes } \\
\text { yes } \\
\text { yes }\end{array}$ & $\begin{array}{l}\text { sandstone } \\
\text { sandstone } \\
\text { sandstone } \\
\text { sandstone }\end{array}$ & $\begin{array}{l}\text { yes } \\
\text { yes } \\
\text { yes } \\
\text { yes }\end{array}$ & $\begin{array}{l}93 \text { (Fig. 3) } \\
93 \text { " " } \\
93 \text { " " } \\
93 \text { " " }\end{array}$ \\
\hline
\end{tabular}


TABLE 26 (continued)

\begin{tabular}{|c|c|c|c|c|c|c|}
\hline Gelant & $\begin{array}{l}\text { Profile } \\
\text { Changed? }\end{array}$ & $\begin{array}{l}\text { Profile } \\
\text { Improved? }\end{array}$ & $\begin{array}{l}\text { Injector } \\
\text { Eractured? }\end{array}$ & Lithology & $\begin{array}{l}\text { Before/After } \\
\text { Injectivity } \\
\text { Given? }\end{array}$ & Reference \\
\hline $\begin{array}{l}\text { acrylamide } \\
\text { monomer }\end{array}$ & yes & yes & $?$ & $?$ & no & 94 (Fig. 5) \\
\hline $\begin{array}{l}\text { complexed } \\
\text { PAM }\end{array}$ & yes & yes & $?$ & shale & no & 95 \\
\hline $\begin{array}{l}\text { complexed } \\
\text { PAM }\end{array}$ & yes & yes & $?$ & sandstone & no & 95 \\
\hline $\mathrm{Zr}-\mathrm{PAM}$ & yes & yes & $?$ & sandstone & yes & 96 (Fig. 4) \\
\hline $\mathrm{Zr}-\mathrm{PAM}$ & yes & debatable & $?$ & sandstone & yes & 96 (Fig. 5) \\
\hline$Z_{r}-P A M$ & yes & yes & $?$ & carbonate & yes & 96 (Fig. 6) \\
\hline Al-PAM & yes & yes & yes & sandstone & yes & 97 (Fig. 4a) \\
\hline Al-PAM & no & no & yes & sandstone & yes & 97 (Fig. 4b) \\
\hline Al-PAM & no & no & yes & sandstone & yes & 97 (Fig. Sa) \\
\hline Al-PAM & no & no & yes & sandstone & yes & 97 (Fig. 5b) \\
\hline $\begin{array}{l}\text { crosslinked } \\
\text { PAM }\end{array}$ & yes & yes & $?$ & $?$ & no & 98 (Fig. 1) \\
\hline aluminate & debatable & debatable & $?$ & $?$ & no & 98 (Fig. 10) \\
\hline
\end{tabular}

Field Work Needed to Resolve the Issue

The issue being discussed here could be settled relatively easily by conducting one or more well designed field tests. Wells should be selected that are known not to be fractured. Several inexpensive methods are available to determine whether or not a well has been fractured, including 1) pressure transient analysis, 2) measuring injectivity (BPD/psi) as a function of in: ‘ction rate, and 3) noting whether the well has ever been axposed to a pressure that was greater than the formation parting pressure. (Incidently, very few of the literature sources here provide this information. ${ }^{31,33,46,65,91-98}$ Also, few of these sources even provide a comparison of injectivity changes before versus after the gel treatments.)

In the well(s) selected for these field tests, at least two distinct zones should be present. These zones should be separated by a sufficient distance ( 10 feet or more) to insure that injection profiles can clearly distinguish flow into the different zones. This separation should also help to minimize complications associated with fluid crossflow between adjacent zones and with flow behind pipe. The permeabilities of the zones should be noticeably different (by at least a factor of two). Ideally, core samples would be available from the different zones 1) to confirm permeabilities and porosities and 2) to allow determination of gel properties in the reservoir rock. 
A number of tests should be conducted both before and after placement of the gel. These include 1) determinirg water injectivity as a function of injection rate (at pressures below the formation parting pressure), 2) determining accurate injection flow profiles, and 3) pressure transient analysis. Injectivity changes should be noted during injection of the gelants. Care should be taken not to fracture the well during the test. For a more elaborate test, inje:tion profiles could also be measured during injection of the gelant.

Results from these different tests can then be compared with predictions from the theoretical analyses. ${ }^{24,25,26}$ Resolution of this issue should significantly improve the success rate for nearwellbore gel treatments.

\section{Summary}

In summary, theoretical analyses indicate that injection profiles usually are not expected to improve significantly in unfractured wells if gels are placed without using zone isolation. The petroleum literature does not provide enough information to confirm or contradict these predictions. In view of the sporadic success of gel treatments, identification of when zone isolation is/is not needed during gel placement is a critical issue. One or more well designed field tests could help to resolve this matter. 


\section{PLACEMENT OF GELS IN PRODUCTION WELLS}

Coping with excess water production is always a challenging task for field operators. The cost of handling and disposing produced water can significantly shorten the economic producing lifo of a well. The hydrostatic pressure created by high fluid levels in the well is also detrimental to oil production.

The two major sources of excess water production are coning and channeling. Water coning is a common problem encountered when a reservoir is produced via a bottom-water-drive mechanism. Fractures and high permeability streaks are the common causes of premature water breakthrough cluring waterfloods. Polymer gels have been applied to many wells to reduce excess water production without adversely affecting oil production. ${ }^{2,34,100-102}$ Hessert and Fleming ${ }^{2}$ reported that polymer gels are particularly effective in suppressing water coning. However, in many cases, gel treatments have not been successful. During gel placement in production welis, much of the gel formulation will enter zones that are responsible for the excess water production. However, some of this fluid may enter into and damage oil-productive strata.

The objectives of this study are to mathematically model the placement of geis in production wells and to examine the potential impact of invasion of gelants into oil-producing zones. Particular attention will be paid to the impact of two phenomena. The first is hysteresis of oil-water relative permeability curves that occurs during the "pump-in, pump-out" sequence used during gel placement in production wells. The second phenomenon is that gels (or polymers) can reduce the relative permeability to water more than to oil.

\section{Theoretical Model}

The first objective of this analysis is to develop a theoretical model for gel placement in production wells. Fractional flow theory is applied to mathematically model the degree of penetration of gelants into zones with different permeabilities during unrestricted injection.

\section{Basic Assumotions}

In examining the placement of gels in production wells, we assume the following:

- All fluids are incompressible and Newtonian.

- Gelant formulations are miscible with water.

- The gelation reaction is slow relative to the placement process.

- Dispersion, retention, and inaccessible pore volume are negligible.

- The resistance factor is independent of permeability.

- There is no mass transfer hetween phases.

- Gravity and capillarity are negligible.

- Darcy's law applies and no fingering occurs during the displacement process.

- Each layer is homogeneous, isotropic, and isothermal.

- The reservoir consists of a number of horizontal noncommunicating layers.

- All layers have the same areal dimensions and share the same injector and producer. (The layers can have different thicknesses.)

For simplicity, we assume that the water and oil relative permeabilities are functions of water saturation only. The fcllowing equations are used throughout this analysis for relative permeability calculations. 103 


$$
\begin{aligned}
& k_{r w}=k_{r w}^{\circ}\left(\frac{S_{w}-S_{w r}}{1-S_{w r}-S_{o r}}\right)^{n_{w}} \\
& k_{r o}=k_{r o}^{\circ}\left(\frac{1-S_{w}-S_{o r}}{1-S_{w r}-S_{o r}}\right)^{n_{0}}
\end{aligned}
$$

\section{Linear Flow}

The behavior of fluid flow in a porous medium during the placement of aqueous gelants is assumed to be the same as that of aqueous polymer solutions during the polymer-flooding process. ${ }^{104}$ Fig. 43 is a schematic diagram of the saturation profile in layer $i$ at a certain instant during the placement process. In linear flow, the instantaneous pressure drop in layer $\mathrm{i}$ between the producer and the injector is

$$
\Delta P_{i}=\frac{\mu_{w} \phi_{i} \bar{S}_{w i}}{k_{i}}\left(F_{r} \int_{0}^{L_{m}} \frac{f_{w i}^{p}}{k_{r w i}} d x+\int_{L_{p,}}^{L_{p h i}} \frac{f_{w i}}{k_{r w i}} d x+\frac{f_{w / i}}{k_{r w I i}}\left(L_{p m}-L_{p k i}\right)+\frac{f_{w I i}}{k_{r w I i}} \Psi_{i} L_{p m}\right) \frac{d L_{p i}}{d t}
$$

where $f_{w}$ and $f_{w}^{p}$ are fractional flow functions of water and gelant, respectively; $L_{p}$ and $L_{p k}$ are the depth of penetration of the gelant front and oil bank, respectively; $L_{p m}$ is a reference distance from the wellbore beyond which the gel treatment has no effect on fluid saturations; and the subscript I denotes the initial condition. $\Psi$ is defined as the ratio of the pressure drop between $L_{p m}$ and the injection well to the pressure drop between the production well and $\mathrm{L}_{\mathrm{pm}}$ just prior to the injection of any gelants (see Ref. 24 for a more detailed discussion of $\Psi$ ). The average water saturation behind the gelant front, $S_{w}$, is determined by using the Welge integration procedure. ${ }^{105}$ Mass balance and the Darcy equation have been used to derive Eq. 44.

Consider the case in which all layers share the same injector and producer and all fluids involved are incompressible. The instantaneous pressure drop across layer 1 is the same as that across layer $\mathrm{i}$. Thus,

$$
\begin{gathered}
\int_{0}^{L_{r}}\left(F_{r} \int_{0}^{L_{p \cdot}} \frac{f_{w i}^{p}}{k_{r w i}} d x+\int_{L_{r+}}^{L_{p h v}} \frac{f_{w i}}{k_{r w i}} d x-\frac{f_{w I j}}{k_{r w I i}} L_{p k i}+\frac{f_{w I i}}{k_{r w I i}}\left(\Psi_{i}+1\right) L_{p m i}\right) d L_{p i}= \\
\frac{\phi_{1} k_{i} \bar{S}_{w 1}}{\phi_{i} k_{1} S_{w i}} \int_{0}^{L_{p 1}}\left(F_{r} \int_{0}^{L_{p 1}} \frac{f_{w 1}^{p}}{k_{r w 1}} d x+\int_{L_{p 1}}^{L_{p h 1}} \frac{f_{w 1}}{k_{r w 1}} d x-\frac{f_{w I 1}}{k_{r w I 1}} L_{r k 1}+\frac{f_{w I 1}}{k_{r w I 1}}\left(\Psi_{1}+1\right) L_{p m}\right) d L_{r 1}
\end{gathered}
$$

The penetration of a gelant into layer $i\left(L_{p i}\right)$ when the oil bank reaches $L_{p m}$ in layer 1 can be determined by solving Eq. 45. Fractional flow theory is applied to determine the frontal position of the oil bank $\left(\mathrm{L}_{\mathrm{pki}}\right)$ relative to that of the gelant front $\left(\mathrm{L}_{\mathrm{pi}}\right)$ and the saturation profile during the displacement process. Analytical solutions for the integrations are difficult to obtain because of the complexity of the functions involved. Instead, the trapezoidal rule is employed to evaluate the integrations numerically. Finally, the secant method is applied to solve for the degree of penetration of the gelant $\left(\mathrm{L}_{\mathrm{pi}} / \mathrm{L}_{\mathrm{p} 1}\right)$. 


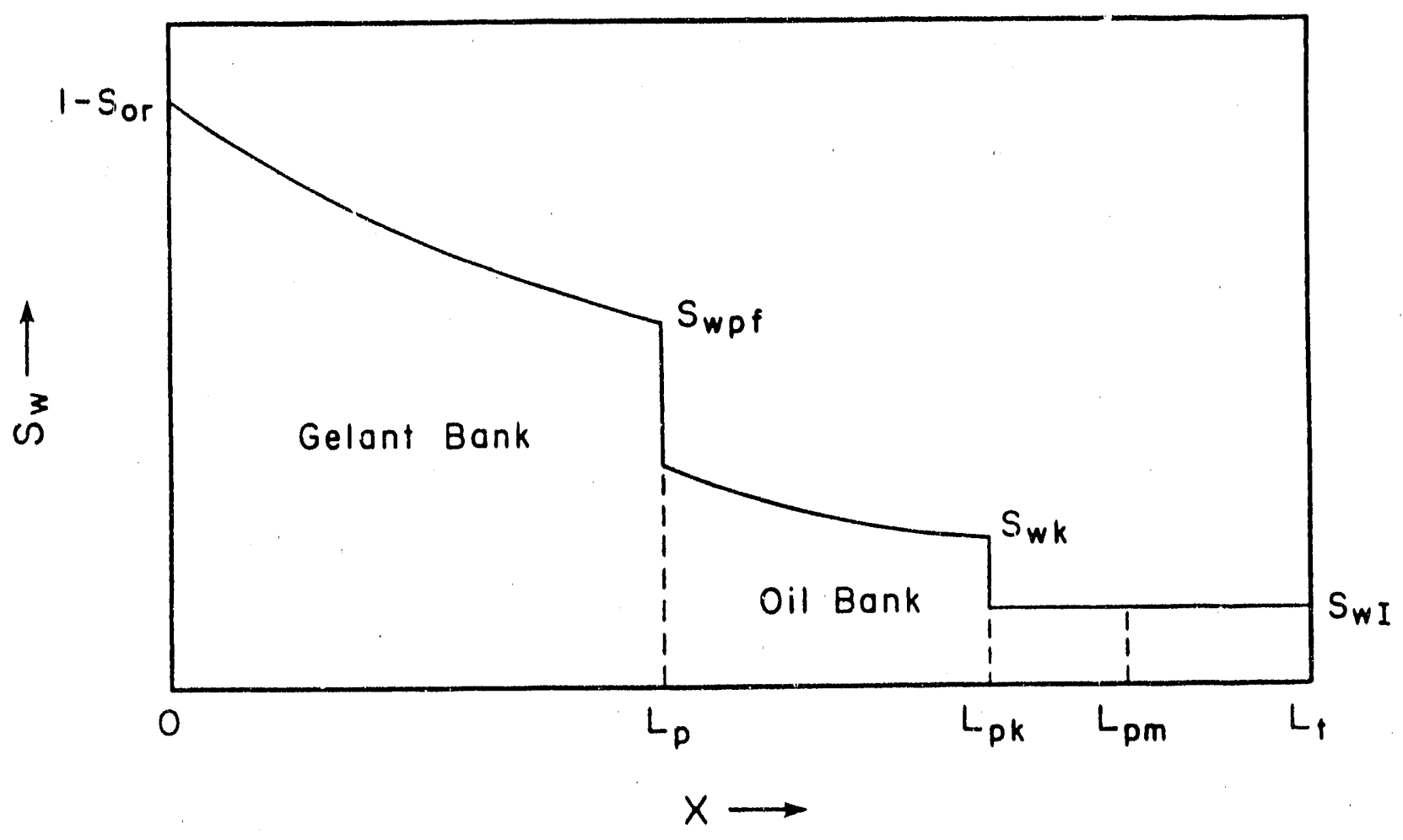

Fig. 43. Saturation Profile in Layer i during the Placement Process. 
For laboratory parallel linear corefloods, the $\Psi$ values for all layers are zero. When the oil bank $\left(L_{p k}\right)$ reaches the outlet of the most-permeable core (core 1), the distance that a gelant has propagated in the less-permeable cores (core i) can be calculated by using the following equation.

$$
\begin{gathered}
\int_{0}^{L_{p w}}\left(F_{r} \int_{0}^{L_{r 1}} \frac{f_{w i}^{p}}{k_{r w i}} d x+\int_{L_{p j}}^{L_{p p r}} \frac{f_{w i}}{k_{r w i}} d x+\frac{f_{w I i}}{k_{r w I i}}\left(L_{t}-L_{p k i}\right)\right) d L_{r i}= \\
\frac{\phi_{1} k_{i} \bar{S}_{w 1}}{\phi_{i} k_{1}} \int^{\bar{S}_{w i}}\left(F_{r} \int_{0}^{L_{p 1}} \frac{f_{w 1}^{p}}{k_{r w 1}} d x+\int_{L_{p 1}}^{L_{1}} \frac{f_{w 1}}{k_{r w 1}} d x\right) d L_{p 1}
\end{gathered}
$$

\section{$\underline{\text { Radial Flow }}$}

A similar procedure is followed in developing the radial model for placement of gels in production wells. The degree of penetration of gelant, $\left(r_{p i}-r_{o}\right) /\left(r_{p 1}-r_{o}\right)$, is determined by solving the following equation.

$$
\begin{aligned}
& \int_{r D 0}^{r D p i}\left(F_{r} \int_{r D 0}^{r_{D p i}} \frac{f_{w i}^{p}}{k_{r w i}} \frac{d r_{D}}{r_{D}}+\int_{r_{D p i}}^{r_{D p k 1}} \frac{f_{w i}}{k_{r w i}} \frac{d r_{D}}{r_{D}}+\frac{f_{w I i}}{k_{r w I i}} \ln \frac{r_{D p m}}{r_{D p k i}}+\frac{f_{w I i}}{k_{r w I i}} \Psi_{i} \ln \frac{r_{D p m}}{r_{D p 0}}\right) d r_{D p i}= \\
& \frac{\phi_{1} k_{i} \vec{S}_{w 1}}{\phi_{i} k_{1} S_{w i}} \int_{r D \cdot}^{r_{D p 1}}\left(F_{r} \int_{r D .}^{r_{D p 1}} \frac{f_{w 1}^{p}}{k_{r w 1}} \frac{d r_{D}}{r_{D}}+\int_{r_{D, 1}}^{r_{D p 11}} \frac{f_{w 1}}{k_{r w 1}} \frac{d r_{D}}{r_{D}}+\frac{f_{w I 1}}{k_{r w 11}} \ln \frac{r_{D p m}}{r_{D p k 1}}+\frac{f_{w I 1}}{k_{r w I 1}} \Psi_{1} \ln \frac{r_{D p m}}{r_{D p 0}}\right) d r_{D p 1}
\end{aligned}
$$

A dimensionless variable, $r_{D}$, defined as

$$
r_{D}=\left(\frac{r}{r_{e}}\right)^{2}
$$

is introduced here so that the solution scheme used in solving Eq. 45 can be applied directly in solving Eq. 47.

For laboratory parallel radial corefloods, the following equation is used to determine the degree of penetration of gelants when the oil bank reaches the outlet of the most-permeable core.

$$
\begin{gathered}
\int_{r_{D 0}}^{r_{D N}}\left(F_{r} \int_{r_{D 0}}^{r_{D N}} \frac{f_{w i}^{p}}{k_{r w i}} \frac{d r_{D}}{r_{D}}+\int_{r_{D p 1}}^{r_{D, w 1}} \frac{f_{w i}}{k_{r w i}} \frac{d r_{D}}{r_{D}}-\frac{f_{w I i}}{k_{r w I i}} \ln r_{D p k i}\right) d r_{D p i}= \\
\frac{\phi_{1} k_{i} \bar{S}_{w 1}}{\phi_{i} k_{1} \bar{S}_{w i}} \int_{r_{D 0}}^{r_{p p 1}}\left(F_{r} \int_{r_{D 0}}^{r_{D p 1}} \frac{f_{w 1}^{p}}{k_{r w 1}} \frac{d r_{D}}{r_{D}}+\int_{r_{D p 1}}^{r_{D p k 1}} \frac{f_{w 1}}{k_{r w 1}} \frac{d r_{D}}{r_{D}}-\frac{f_{w I 1}}{k_{r w I 1}} \ln r_{D p k 1}\right) d r_{n_{p 1}}
\end{gathered}
$$

In this study, the degree of penetration of gelants is determined when the oil bank in the most-permeable layer reaches $\mathrm{r}_{\mathrm{pm}}\left(\mathrm{L}_{\mathrm{pm}}\right)$ or the outlet of the core. However, if no oil bank forms in the most-permeable layer (e.g., if the most-permeable layer is watered-out), then the degree of penetration is determined when the gelant front reaches $\mathrm{r}_{\mathrm{pm}}\left(\mathrm{L}_{\mathrm{pm}}\right)$ or the outlet of the core. In some unusual cases $\left(k_{1} / k_{i} \sim 1\right)$, the invasion of gelants into a given less-permeable layer can be slightly greater than that into the most-permeable layer. The degree of penetration of gelants, in this case, 
is determined when the oil bank in the less-permeable layer reaches $\mathrm{r}_{\mathrm{pm}}\left(\mathrm{L}_{\mathrm{pm}}\right)$ or the outlet of the core. In all cases that we studied, the degree of penetration of gelants is not sensitive to the choice of $r_{p m}$ $\left(L_{\mathrm{pm}}\right)$ or the position of the gelant front. Appendixes $F$ and $G$ provide Fortran coding for what is referred to in this paper as the "theoretical model" (Appendix F for radial flow, and Appendix $G$ for linear flow).

\section{Linear vs. Radial Corefloods}

To quantify the impact of the factors affecting the degree of penetration of gelants, consider injection of a gelant in to a number of parallel homogeneous cores of equal length from a common injection port. The most-permeable core is completely watered-out, and the rock and fluid properties are summarized in Table 27.

The degree of penetration of gelant into a less-permeable core (core $\mathrm{i}$ ) is presented in Fig. 44. (These results were generated by using Eqs. 46 and 49.) As expected, Fig. 44 shows that the degree of penetration of a gelant into the less-permeable cores decreases with increasing permeability contrast. Figure 44 also demonstrates that the degree of penetration of a gelant into the less-permeable cores is less in linear f!uw than in radial flow. This fact was also noted for gel placement in injection wells. ${ }^{24}$ It is partly the reason why zone isolation is more likely to be needed during gel placement in radial flow than in linear flow.

Since the geometry surrounding an unfractured production well is radial rather than linear, the following analysis will focus on radial cases only. Unless otherwise noted, the rock and fluid properties of the examples involved in the following analysis are described in Table 27.

A basic principle in polymer flooding is that an increase in resistance factor will increase the degree of penetration into the less-permeable layer. ${ }^{24}$ Figure 44 demonstrates that this principle is generally valid in production well treatments. However, this trend is moderated significantly at low oil-water viscosity ratios.

A comparison of Fig. 44 in this report and Fig. 1 from Ref, 24 reveals that the degree of penetration of a gelant into a given less-permeable layer in production well treatments is similar to that in injection well treatments. ${ }^{24}$. Hence, the need for zone isolation is of concern during gel placement in production wells.

Fluid flow during gel placement in production wells can be characterized using fractional flow theory. The factors affecting fractional flow (such as the oil-water viscosity ratio, the water/oil relative permeability curves and the fluid saturations in the porous medium) can also affect the degree of penetration of gelants.

As can be seen in Fig. 45, the degree of penciration of a gelant into a given less-permeable layer increases with decreasing oil-water viscosity ratio. However, the ef fect becomes less significant at low oil-water viscosity ratios.

Figure 46 shows that the degree of penetration is fairly insensitive to the end-point relative permeability to water $\left(k_{\mathrm{rw}}^{0}\right)$. Figure $4 \dot{c}$ also demonstrates that gelants penetrate less into the less-permeable layer as the initial water saturation increases in the most-permeable layer. In examining the effect of water/oil relative permeabilities on the degree of penetration of gelants, we assume that the water relative permeability curve shifts proportionately with the changing end-point values. The oil relative permeability curve remains unchanged throughout this analysis. 


$$
\begin{array}{ll}
k_{r w}^{\prime}=0.1 & k_{r o}^{\prime}=1 \\
\phi_{1} / \phi_{1}=1 & \mu_{o} / \mu_{w}=10 \\
S_{w r}=0.2 & S_{o r}=0.2 \\
S_{w 1}=0.8 & S_{w i}=0.2 \\
F_{r}=1 &
\end{array}
$$

For radial coreflood cases:

$r_{0}=0.5 \mathrm{ft}$

$$
r_{e}=50 \mathrm{ft}
$$

$k_{r w}^{\prime}=0.1$
$S_{w r}=0.2$
$S_{w 1}=0.8$
$\phi_{1} / \phi_{i}=i$

Well pattern:

$r_{0}=0.5 \mathrm{ft}$

$\mathrm{r}_{\mathrm{p} 1}=50 \mathrm{ft}$ $k_{\text {ro }}^{\prime}=1$

$S_{\text {or }}=0.2$

$S_{w i}=0.2$

20-acre 5-spot

$r_{e}=372.4 \mathrm{ft}$

$r_{p m}=100 \mathrm{ft}$

TABLE 29

Parameters Used When Comparing the Theoretical and Numerical Models
$k_{\mathrm{rw}}^{\prime}=0.1$
$k_{\mathrm{ro}}^{\prime}=1$
$\phi_{1} / \phi_{i}=1$
$\mu_{\mathrm{o}} / \mu_{\mathrm{w}}=10$
$S_{\mathrm{wr}}=0.2$
$\mathrm{S}_{\mathrm{or}}=0.2$
$S_{w 1}=0.8$
$\mathrm{S}_{\mathrm{wi}}=0.5$ 


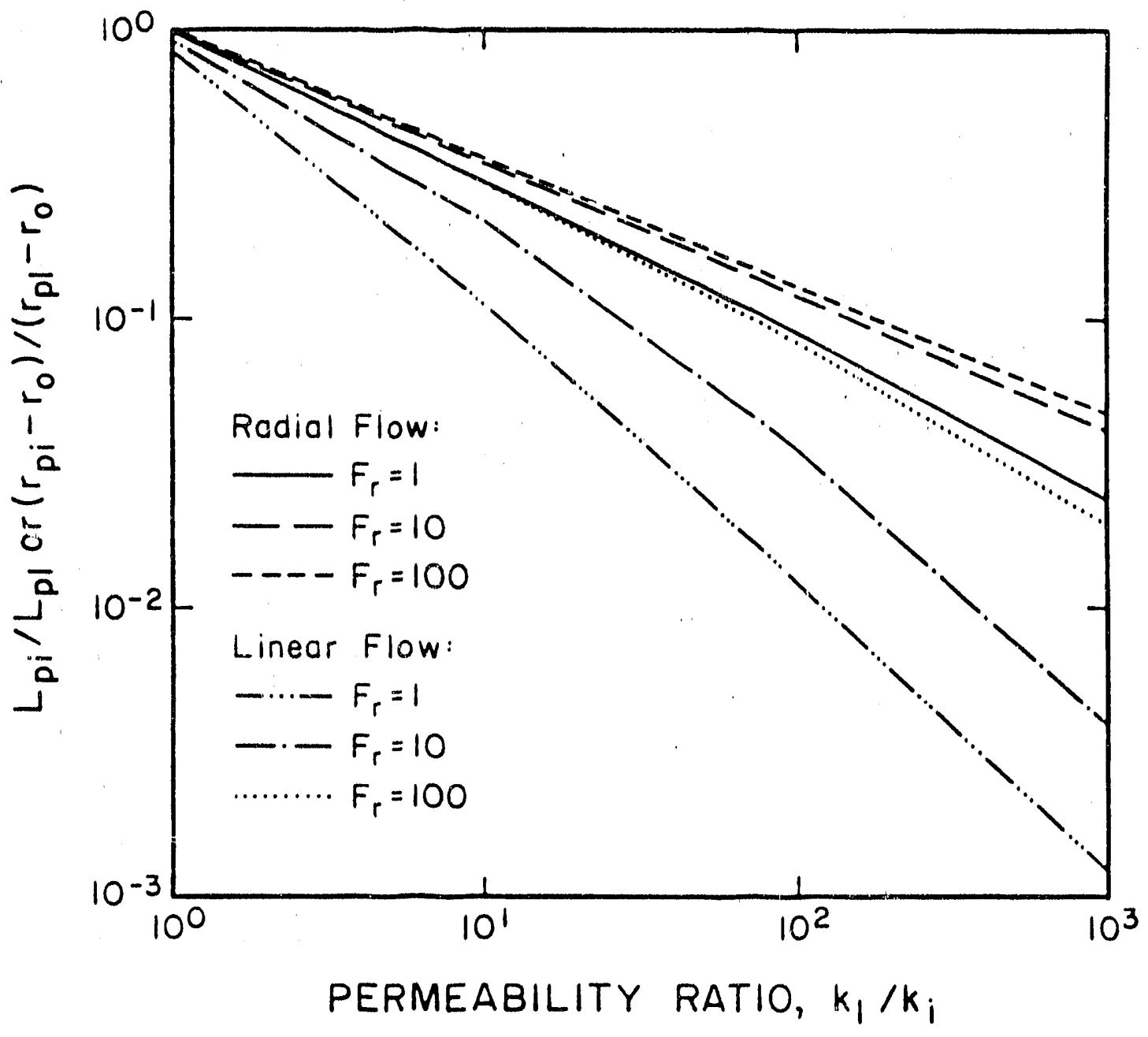

Fig. 44. Radiai vs. Linear Parallel Corefloods $\left(\mu_{0} / \mu_{w}=10\right)$; Degree of Penetration into Core $i\left(r_{p 1}=100 r_{0}, \phi_{1}=\phi_{i}\right)$. 


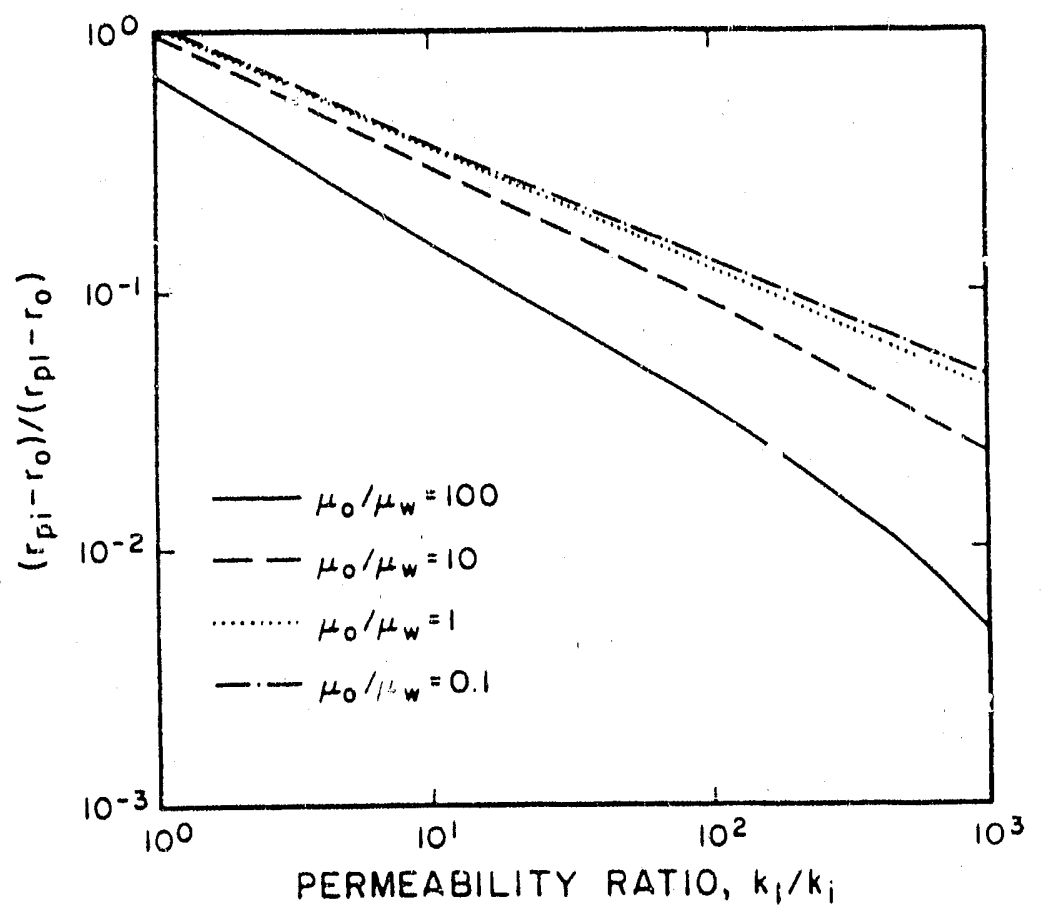

F:g. 45. Effect of Oil/Water Viscosity Ratio on Degree of Penetration into Core $i$ (Radial Flow, $F_{r}=1$ ).

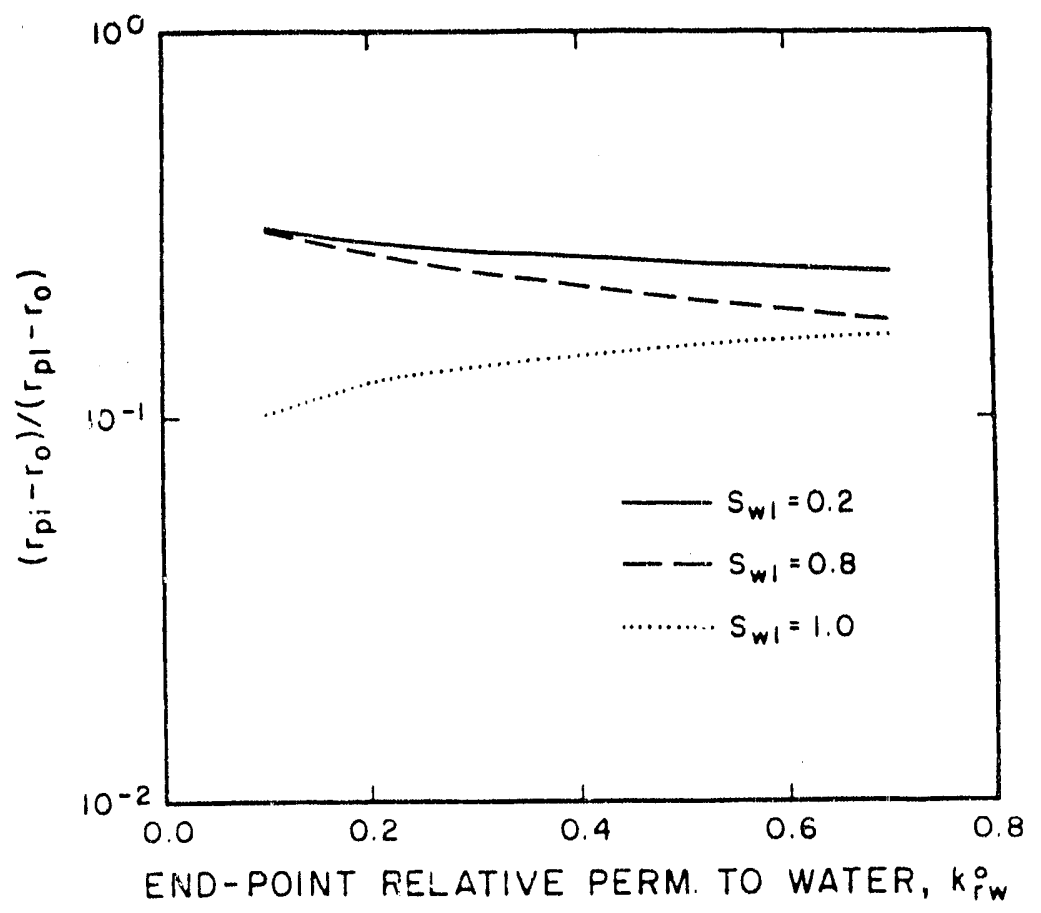

Fig. 46. Degree of Penetration into Core i; Effects of End-Point Permeability to Water and $S_{w 1}$ $\left(\mathrm{k}_{1} / \mathrm{k}_{\mathrm{i}}=10, \mathrm{~S}_{\mathrm{wi}}=0.2\right)$. 


\section{Flow in Reservoirs}

In actual field applications in unfractured wells, gelants usually penetrate a relatively short distance into the formation (e.8., $50 \mathrm{ft}$ into the most-permeable layer). Thus, in this study, the greatest distance that gelants penetrate into the most-permeable layer $\left(r_{p 1}\right)$ is set at $50 \mathrm{ft}$ from the wellbore. Since an oil bank of ten precedes the gelant front, a gel treatment can af fect fluid saturations at distances beyond the greatest depth of penetration of gelant. A distance, $r_{\mathrm{pm}}$, will be chosen such that the gel treatment never has any effect on fluid saturations at distances greater than $r_{p m}$ from the wellbore. Somewhat arbitrarily, we will assume that $r_{\mathrm{pm}}$ has a value of $100 \mathrm{ft}$.

For the case of waterflood in a five-spot pattern, the factor $\Psi$ in Eq. 47 can be approximated by the following equation. ${ }^{106,107}$

$$
\Psi_{i}=\frac{\ln \left(\frac{r_{a}}{r_{p m}}\right)+\frac{1}{M} \ln \left(\frac{r_{a}}{r_{0}}\right)}{\ln \left(\frac{r_{p m}}{r_{0}}\right)}
$$

For simplicity, the water front is assumed to coincide with the external drainage radius of the reservoir. However, the $\Psi$ value is not sensitive to the position of the water front. According to Eq. 50, $\Psi$ is strongly dependent upon the water-oil mobility ratio. The value of $\Psi$ is fairly insensitive to other factors, such as well spacing and the choice of $\mathrm{r}_{\mathrm{pm}} \cdot{ }^{24}$

Consider an example where the most-permeable layer is completely watered-out (Table 28 on page 92). Since water is the only mobile fluid in the most-permeable layer, the mobility rativ, $M$, in Eq. 50 is equal to 1. Hence, in the most-permeable layer, $\Psi_{1}$ is about 1.5. However, in the less-permeable layers, $\Psi_{i}$ could have any value (in this example) in the practical range from 0.3 to 16 , depending on the water-oil mobility ratio.

Figure 47 illustrates the effect of $\Psi$ on the degree of penetration of gelants. The results presented in Fig. 47 were generated by using Eq. 47. As can be seen in Fig. 47, the degree of penetration of gelants into the less-permeable layer increases with increasing $\Psi_{j}$ value. However, the effect is not as great as that in the injection well treatment cases. ${ }^{24}$ The degree of penetration becomes insensitive to the $\Psi_{\mathrm{i}}$ value for high gelant resistance factors $\left(F_{\mathrm{r}}\right)$.

\section{Numerical Model}

To verify the solutions from Eqs. 44, 45, 46, 47, and 49, a numerical-simulation model was developed. The IMplicit Pressure Explicit Saluration method (IMPES) and upstream weighting on mobilities were used. Good agreement between numerical solutions and solutions from Eqs. 44-49 was found.

In the simulation, each layer was discretized into subdomains (blocks or nodes) with a fixed pressure drop across layers. Pressures profiles were solved with given time intervals while saturations were updated at every time step. During gelant injection, the gelant was assumed to displace all water behind the gelant-water interface. Therefore, the interface could be determined at each time step as long as the total injection was known. Numerical dispersion and oscillation were found near the shock - front areas and were minimized by using small time intervals-allowing nodal saturation changes no more than 0.1 at each time step. Appendix $H$ provides a more detailed description of the formulation of the nurnerical model. 


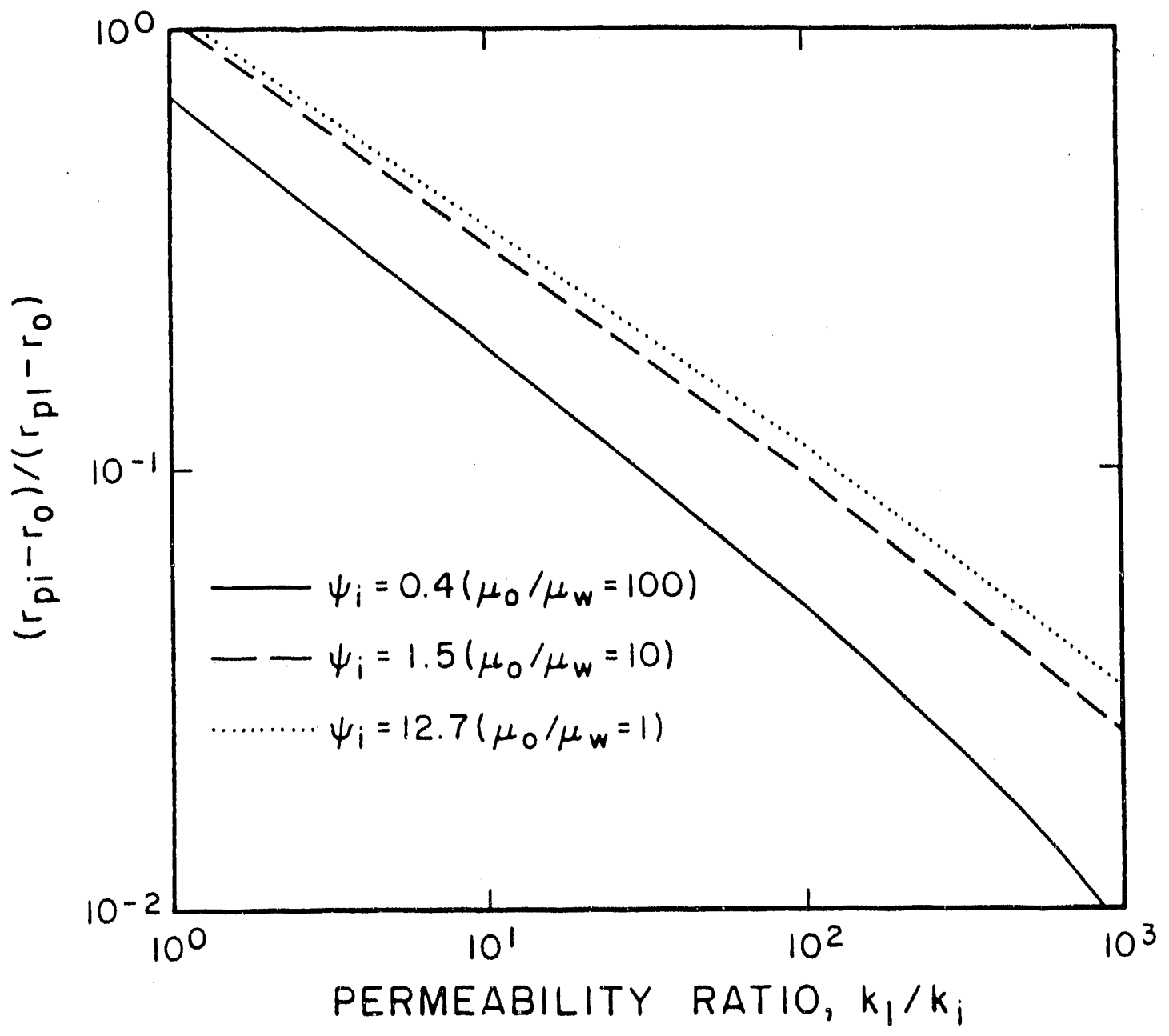

Fig. 47. Effect of $\Psi_{i}$ Variations $\left(F_{r}=1\right)$ on Degree of Penetration into Layer $i$. 
Generally, one hundred grid blocks were assigned to each layer using equal spacing between nodes. In radial cases, the differences in the predictions from the theoretical model and the numerical model were greater than those found in linear cases. Differences beween the theoretical and the numerical model become more pronounced as the permeability ratio increases. (An exception to this occurs when $F_{r}=1$, in which case, the differences appear to decrease with increased permeability ratio.) These differences can be reduced by increasing the number of nodes in the numerical model. For example, when $k_{1} / k_{i}=100$ and $F_{r}=100$, the differences in the predictions from the two models are reduced from $40.5 \%$ to $17.2 \%$ when the number of nodes per layer is increased from 100 to 1000 , respectively. Figure 48 compares results from the theoretical model and the numerical model for $F_{r}$ $=1, F_{r}=2$, and $F_{r}=100$ cases. When $F_{r}=10$, the penetration ratio is very close to that for $F_{r}=100$. Please refer to Table 29 (on page 92) for the rock and fluid properties involved in this example.

\section{Relative Permeability Changes After Treatment}

Many researchers ${ }^{56-60}$ have reported that water-soluble polymers reduce permeability to water significantly more than they reduce permeability to oil. Needham et al. ${ }^{56}$ also reported that crosslinking of water-soluble polymers with multivalent cations results in a greater and more permanent reduction of water velative permeability.

In examining the potential impact of this selective permeability reduction, we will initially assume that the permeability to oil is not affected by the gel treatment. The water relative permeability is, however, decreased in proportion to the residual resistance factor $\left(F_{r r}\right)$ of the particular gelant involved.

For near-wellbore gel treatments in unf ractured production wells, gelants penetrate a relatively short distance into the formation (e.8., $50 \mathrm{ft}$ into the most-permeable zone). The water saturation, the water and oil relative permeability curves, and the fluid fractional flow curves remain unchanged in the region that is not contacted by the gelant. However, the reduced water permeability in the gel-treated region reduces production of all fluids from the treated zone. This can be understood by considering the following example.

Consider a reservoir that consists of a single stratum. Let the rock properties and relative permeabilities be described by the parameters listed in Table 30 (on page 100). As shown in Fig. 49, the water relative permeability curve is shifted downward as a result of a gel treatment while the oil relative permeability curve remains unchanged. This shift also changes the fractional flow curve governing the fluid flow in the treated region (Fig. 50). Since gels usually only invade a relatively short distance into the formation during treatments, it is reasonable to expect that a local steady-state flow condition can be achieved in the vicinity of the treated region after the resumption of flow. Under steady-state conditions, the fractional flow of water in the gel-treated region must be the same as that in the untreated region (a consequence of mass balance). Therefore, the water saturation in the gel-treated region must shift (as illustrated in Fig. 50) from 0.5 (the initial water saturation before treatment) to 0.625 after treatment to maintain the same fractional flow of water under the new fractional now curve. This increase in water saturation after treatment can cause significant reduction in oil relative permeability in the treated region. As indicated in Fig. 49, the oil relative permeability is reduced from 0.125 before treatment to 0.025 af ter treatment as a result of the water saturation increase. The impact of the reduced oil permeability on oil productivity will be discussed in the next section. 


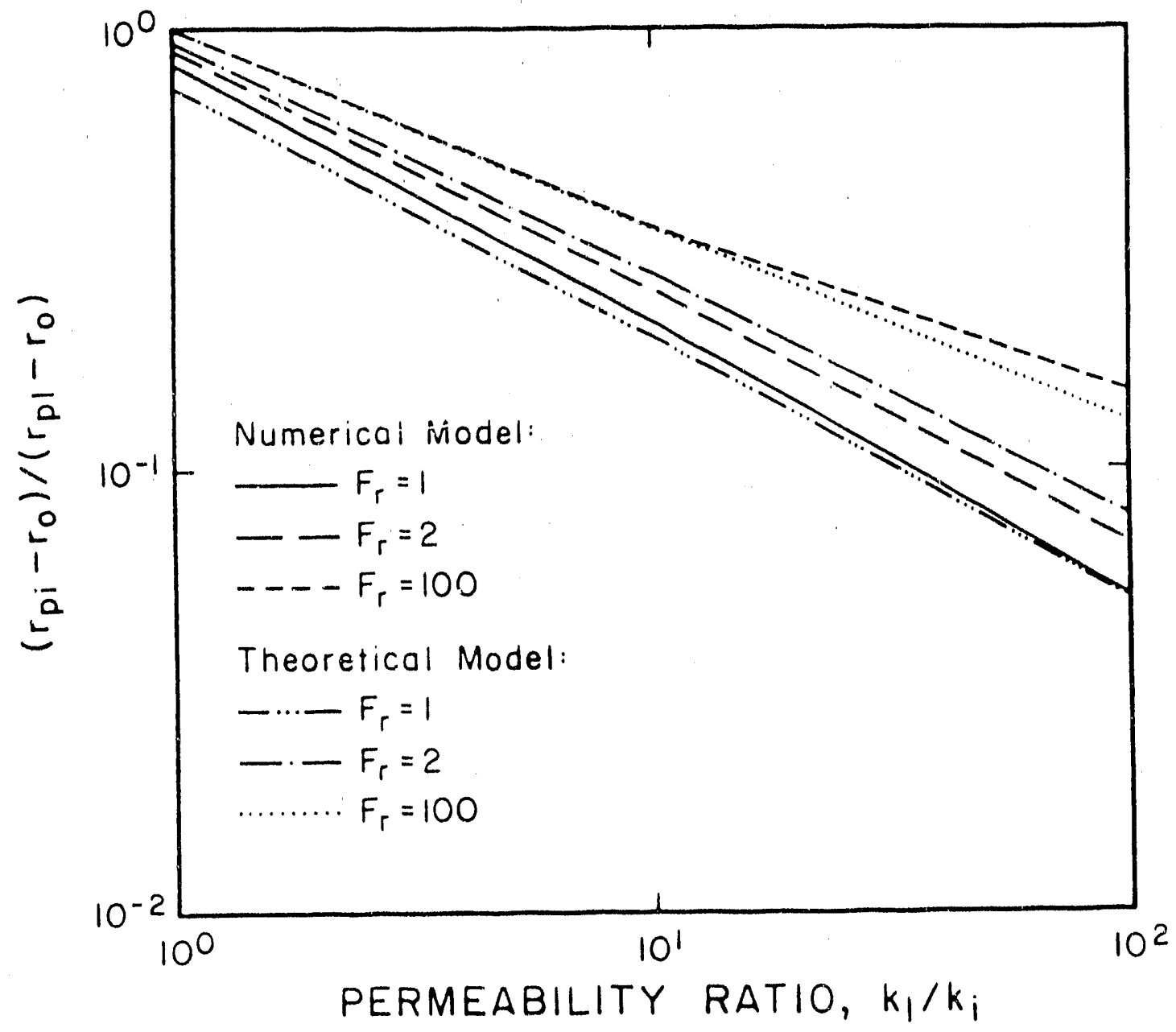

Fig. 48. Theoretical Model vs. Numerical Model; Degree of Penetration into Core i: Radial Flow. 


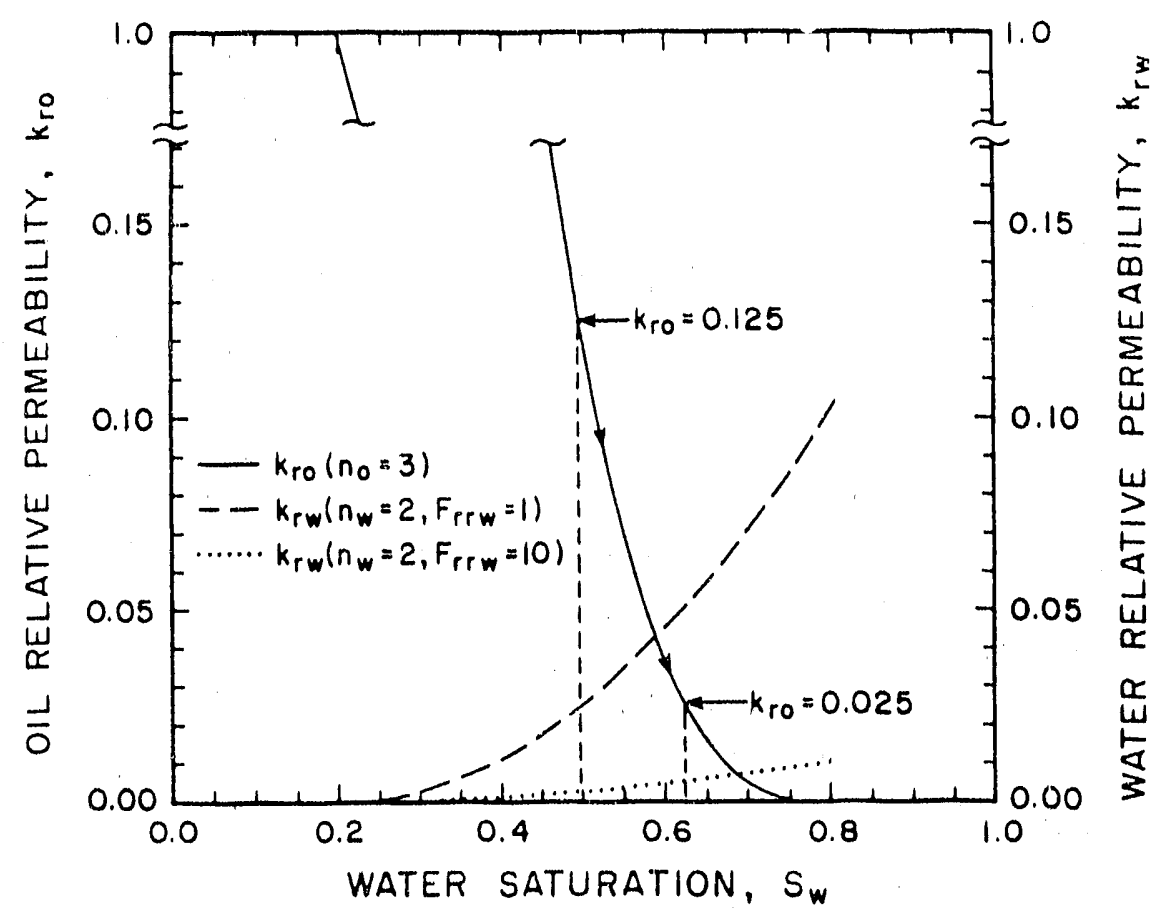

Fig. 49. Oil and Water Relative Permeability Curves Before and After Gel Treatment.

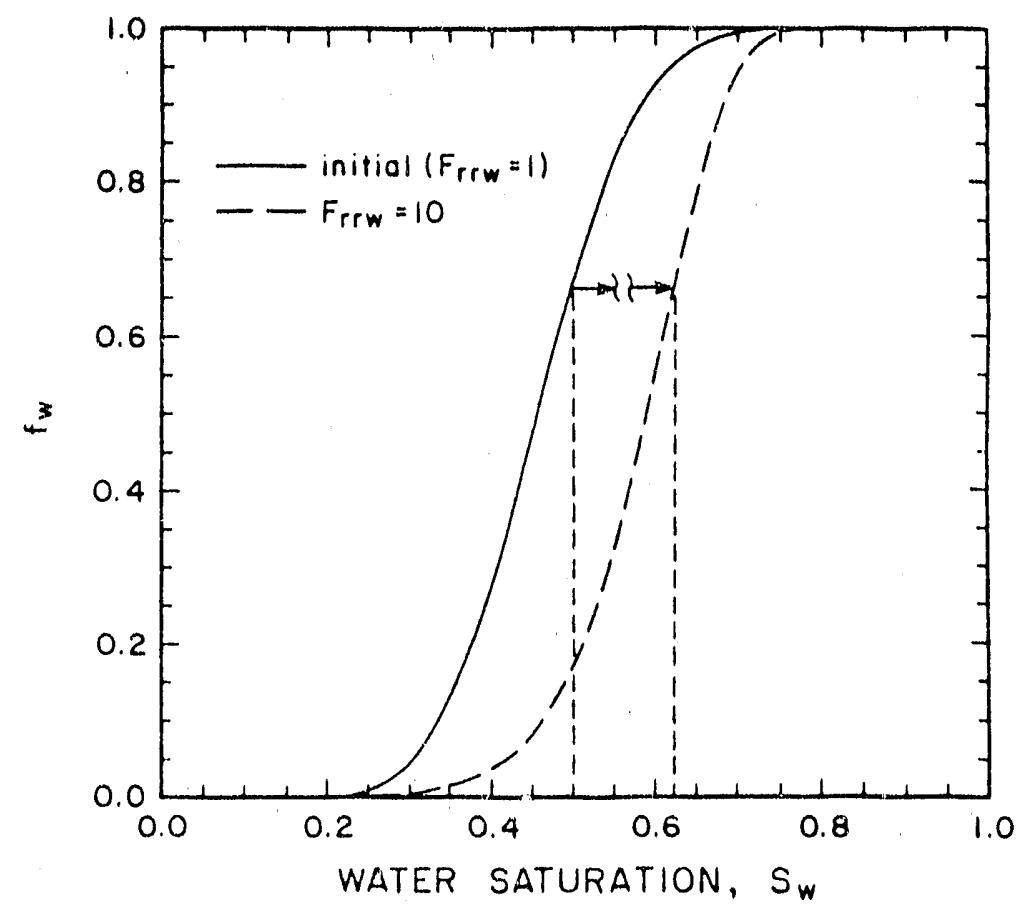

Fig. 50. Water Fractional Flow Curves Before and After Gel Treatment. 


$\begin{array}{ll}\mathrm{k}_{\mathrm{rw}}^{\prime}=0.1 & \mathrm{k}_{\mathrm{ro}}^{\prime}=1 \\ \mathrm{n}_{\mathrm{w}}=2 & \mathrm{n}_{\mathrm{o}}=3 \\ \mathrm{~S}_{\mathrm{wr}}=0.2 & \mathrm{~S}_{\mathrm{or}}=0.2 \\ \mathrm{~S}_{\mathrm{wi}}=0.5 & \mathrm{~F}_{\mathrm{r}}=1 \\ \mathrm{~F}_{\mathrm{rww}}=10 & \mathrm{~F}_{\mathrm{rro}}=1 \\ \mu_{\mathrm{o}} / \mu_{\mathrm{w}}=10 & \end{array}$

$\begin{array}{lc}\mathrm{k}_{\mathrm{rw}}^{\prime}=0.1 & \mathrm{k}_{\mathrm{ro}}^{\prime}=1 \\ \mathrm{n}_{\mathrm{w}}=2 & \mathrm{n}_{\mathrm{o}}=3 \\ \mathrm{n}_{\mathrm{w}}^{\prime}=6 \text { (hysteresis) } & \mathrm{n}_{0}^{\prime}=3 \\ \mathrm{~S}_{\mathrm{wr}}=0.2 & \mathrm{~S}_{\mathrm{or}}=0.2 \\ \mu_{\mathrm{o}} / \mu_{\mathrm{w}}=10 & \mathrm{~F}_{\mathrm{r}}=1 \\ \text { Well pattei } \mathrm{n}: & 20 \text {-acre 5-spot } \\ \mathrm{r}_{\mathrm{o}}=0.5 \mathrm{ft} & \mathrm{r}_{\mathrm{e}}=372.4 \mathrm{ft} \\ \mathrm{r}_{\mathrm{pm}}=100 \mathrm{ft} & \end{array}$

$\mathrm{k}_{\mathrm{rw}}^{\prime} \mathrm{ax} 0.1$

$\mathrm{n}_{\mathrm{w}}=2$

$S_{w r}=0.2$

$\mu_{\mathrm{o}} / \mu_{\mathrm{w}}=10$

$F_{\text {rww }}=10$

Well pattern:

$r_{0}=0.5 \mathrm{ft}$

$r_{p o n}=100 \mathrm{ft}$ $k_{\text {ro }}^{\prime}=1$

$\mathrm{n}_{\mathrm{o}}=3$

$S_{\text {or }}=0.2$

$\mathrm{F}_{\mathrm{r}}=1$

$F_{\text {ro }}=1$

20-acre 5-spot

$r_{e}=372.4 \mathrm{ft}$ 


\section{Productivity Loss After Treatament}

The goal of near-wellbore gel treatments in oil production wells s to reduce water production without sacrificing oil production. For the case of constant pressure di

the fraction of the original water productivity in zone $i$ that remains after the gel treatment is

$$
\frac{q_{w i}}{q_{w o i}}=\frac{\left(\Psi_{i}+1\right) \ln \left(\frac{r_{p m}}{r_{0}}\right)}{\frac{k_{r w i-1}}{k_{r_{w+1}}} \ln \left(\frac{r_{p i}}{r_{0}}\right)+\ln \left(\frac{r_{p m}}{r_{p 1}}\right)+\Psi_{i} \ln \left(\frac{r_{p m}}{r_{0}}\right)},
$$

and the fraction of the original oil productivity in zone $i$ that remains after gel treatment is

$$
\frac{q_{o i}}{q_{o o i}}=\frac{\left(\Psi_{i}+1\right) \ln \left(\frac{r_{p m}}{r_{0}}\right)}{\frac{k_{\text {rai }}}{k_{r o p i}} \ln \left(\frac{r_{p i}}{r_{o}}\right)+\ln \left(\frac{r_{p m}}{r_{p i}}\right)+\Psi_{i} \ln \left(\frac{r_{p m}}{r_{0}}\right)}
$$

where $k_{r w i}$ and $k_{\text {roti }}$ are the water and oil relative permeabilities in the treated region of zone $i$. The relative permeabilities in the treated region can be calculated based on the new oil-water relative permeability curves and the new oil and water saturations. Thus, the productivity loss in a given zone depends on the degree of penetration of the gelant, on the $\Psi$ value of the zone, on the changes in the oil and water relative permeabilities, and on the resulting changes in saturations in the treated region.

As mentioned earlier, the fractional flow of water and oil from a given zone must remain fixed. As a consequence, if the water productivity from a given zone changes, then the oil productivity from that zone must change by the same fraction. In other words, the right"- and left-hand sides of Eqs. 51 and 52 are all equal.

\section{Hysteresis of Qiland Water Relative Permeability Curves}

The relative permeability of a given phase is of ten both path/and history dependent. ${ }^{107}$ Jones and Roszei:: ${ }^{108}$ reported that the relative permeability to oil is relatively unchanged from the imbibition values. However, for a water-wet core, the water relative permeability curves during imbibition (waterflooding) can be different than those during drainage (oilflooding after waterflooding). Since gel treatments in production wells involve both an imbibition cycle (the in jection of an aqueous gelant) and a drainage cycle (the resumption of oil production), the effect of this hysteresis should be considered.

To examine the impact of this hysteresis, consider injection of a small volume of water into an unfractured production well (Table 31 on page 100). The relative permeability curves of both the imbibition and drainage cycles are presented in Fig. 51 .

After production is resumed and a steady state is achieved, the water saturation in the region contacted by the injection water must be increased to maintain the level of water fractional flow. However, the increase in water saturation would reduce the oil relative permeability and impair the oil productivity of the oil productive zones. Figure 52 illustrates the effect of the hysteresis of relative permeability curves on the productivity loss at various initial water saturations. For this example, a single layer is used, and the waterfront is allowed to penetrate $50 \mathrm{ft}$ into the layer. (Remember that oil productivity and water productivity must experience the same fractional change.) 


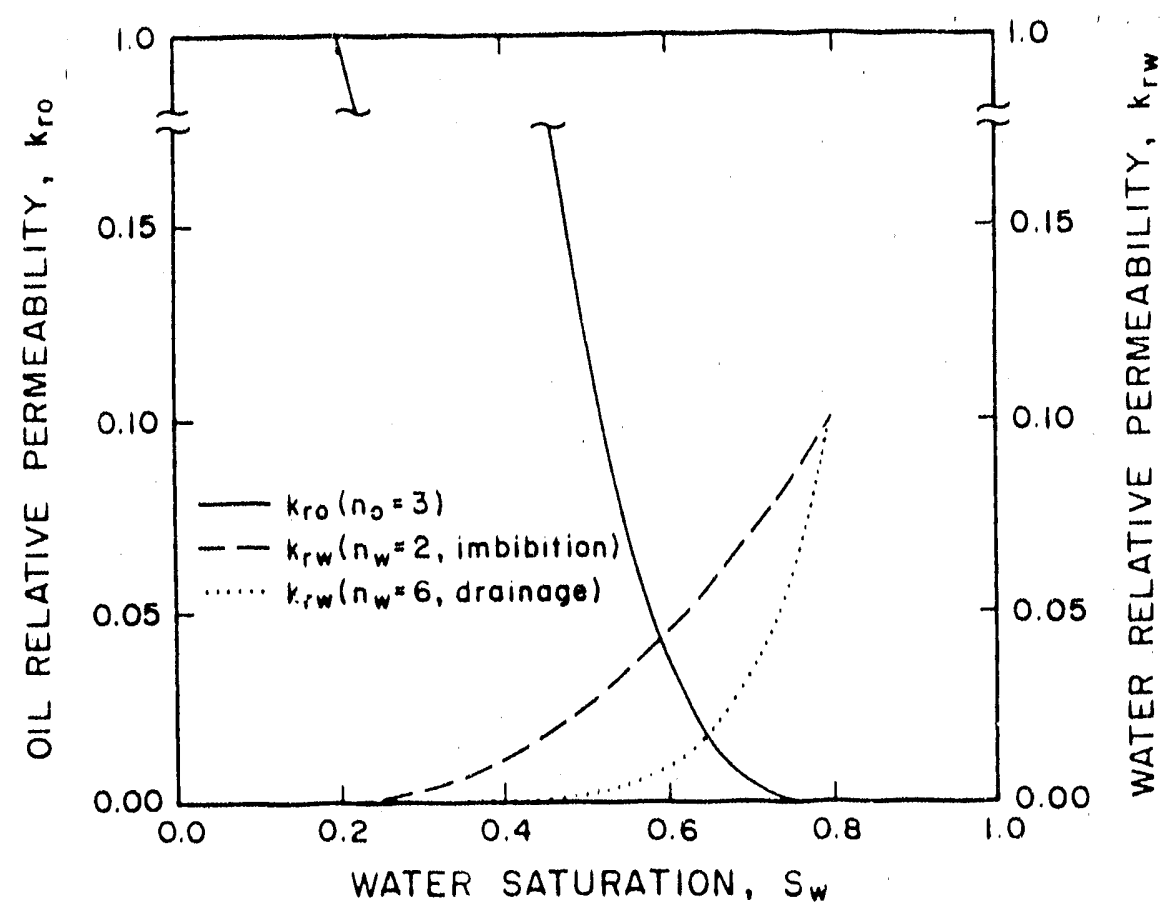

Fig. 51. Oil and Water Relative Permeability Curves with/without Hysteresis.

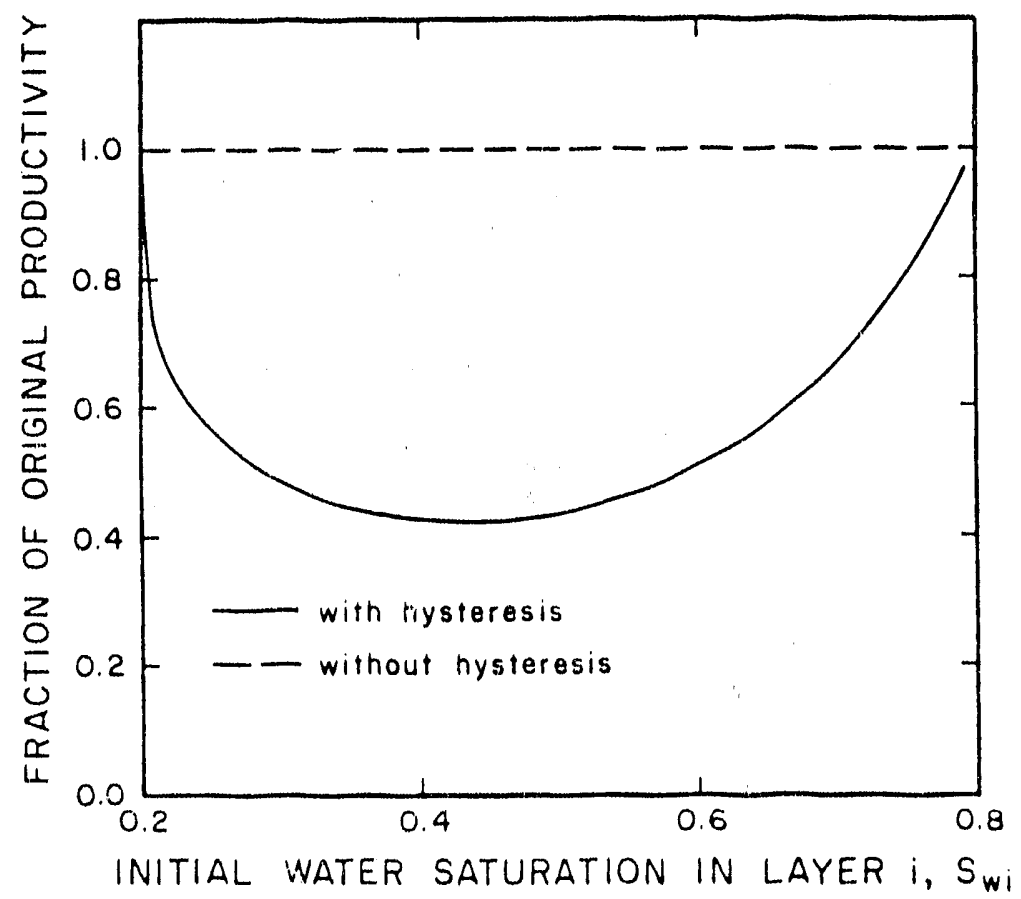

Fig. 52. Ef fect of Hysteresis of Relacive Permeability Curves on Productivity Loss (depth of water penetration $=50 \mathrm{ft}$, radial flow). 
As can be seen in Fig. 52, hysteresis of the water relative permeability eurve can cause significant damage to oil productivity for most water saturations. Thus, hysteresis of relative permeability curves should be considered when applying gel treatments.

\section{Productivity Loss After Gelation}

Since hysteresis alone can impair oil productivity, the effects of a gel treatment on productivity loss with and without hysteresis are both examined. Consider the case where no hysteresis is involved. Table 32 (on page 100) is a list of the parameters used in the example. The relative permeability curves and the corresponding fractional flow curves are shown in Fig. 49 and Fig. 50, respectively. The oil and water productivity losses after gel treatment (expressed as the fraction of the original productivity) are plotted against the depth of penetration of the gelant into the formation in Fig. 53a. Since the material balance dictates that the level of water fractional flow in a given zone in the formation remain unchanged after treatinent, the fraction of productivity loss of oil for a given zone after treatment is the same us that of water after treatment.

A key point to be made from Fig. $53 \mathrm{a}$ and $53 \mathrm{~b}$ is that the gels will cause some loss of oil productivity, unless the oil saturation is very high. This occurs even though the gel did not affect the oil permeability in the gel-treated region. Figure 53a also demonstrates that the productivity loss af ter treatment increases as the water saturation increases in the zone of interest. As expected, the productivity after treatment declines with increasing depth of penetration of gelant.

The productivity loss after gel treatment with 1 ysteresis is illustrated in Fig. 53b. A comparison of Fig. 53a and Fig. $53 \mathrm{~b}$ reveals that, as expected, the hysteresis of relative permeability curves further impairs oil productivity.

\section{Fractured Systems}

In this section, our analysis will focus on vertically fractured wells. The fractures are assumed to extend through all of the productive zories. These productive zones are separated by impermeable layers (except at the fracture face). Since the length of a vertical fracture is generally much longer than the wellbore radius, and the "permeability" of a fracture is much greater than that of the porous medium, the flow of a gelant from the fracture face into the rock matrix is considered linear.

Eqs. 15 and 50 can be applied to solve for the degree of penetration of gelants into the rock matrix that is atjacent to a fracture face. However, $L_{p i}$ is now defined as the distance from the fracture face into the formation that a gelant has propagated in layer $i$ when the oil bank reaches $L_{p m}$ in the most-permeable layer (layer 1). Also, $r_{0}$ and $r_{p m}$ in Eq. 50 are replaced by $L$ and $L+L_{p m}$, respectively.

An example similar to the one used in the previous section for unfractured wells is used here to examine the placement of gels in vertically fractured wells (Table 32 on page 100). The degree of penetration of a gelant is plotted against the permeability ratio at different $\Psi$ values in Fig. 54. Figure 54 shows that the degree of penetration of a gelant increases as the $\Psi$ value increases. A comparison of Fig. 47 and Fig. 54 reveals that the degree of penetration of gelants in the less-permeable layers is generally less in the fractured cases. Thus, the need for zone isolation is less when treating vertically fractured wells than when treating unfractured wells. 


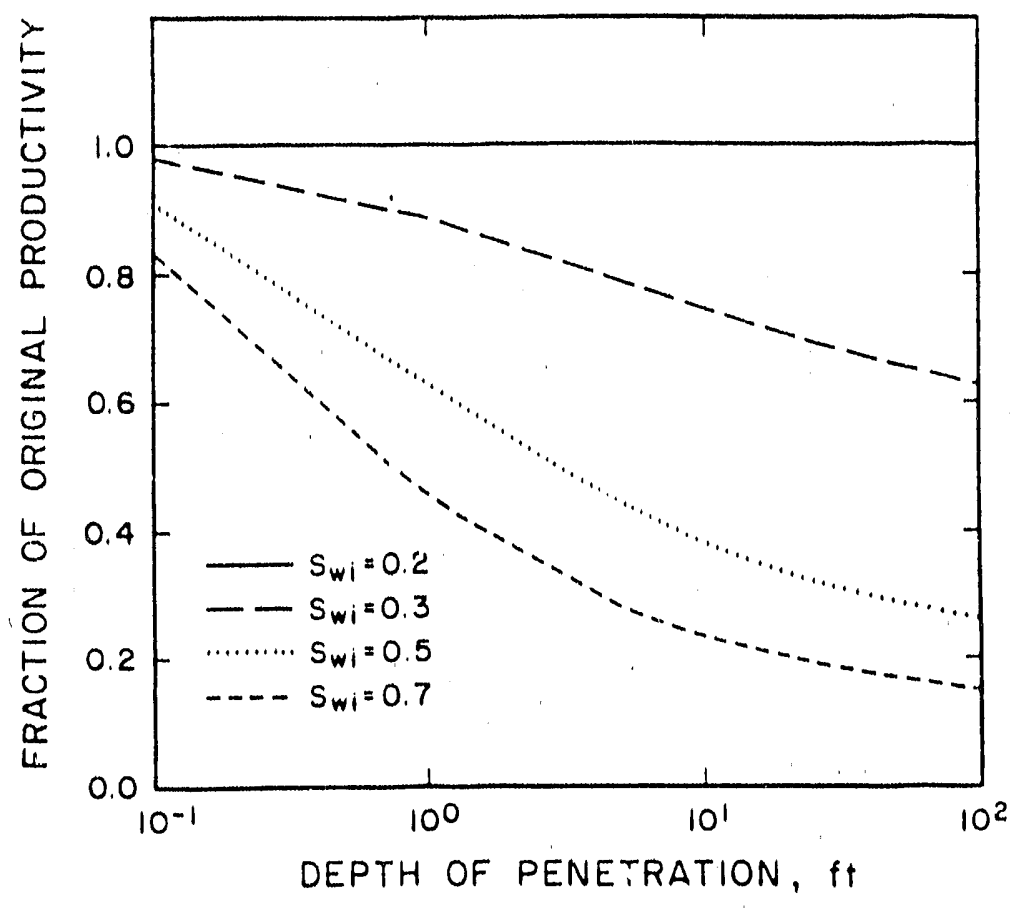

Fig. 53a. Ef fects of Depth of Penetration and $S_{w i}$ on Productivity Retained af ter Gel Treatment $\left(F_{\text {rrw }}\right.$ $=10, F_{\text {ro }}=1$, no hysteresis, $n_{w}=2, n_{0}=3$, radial flow).

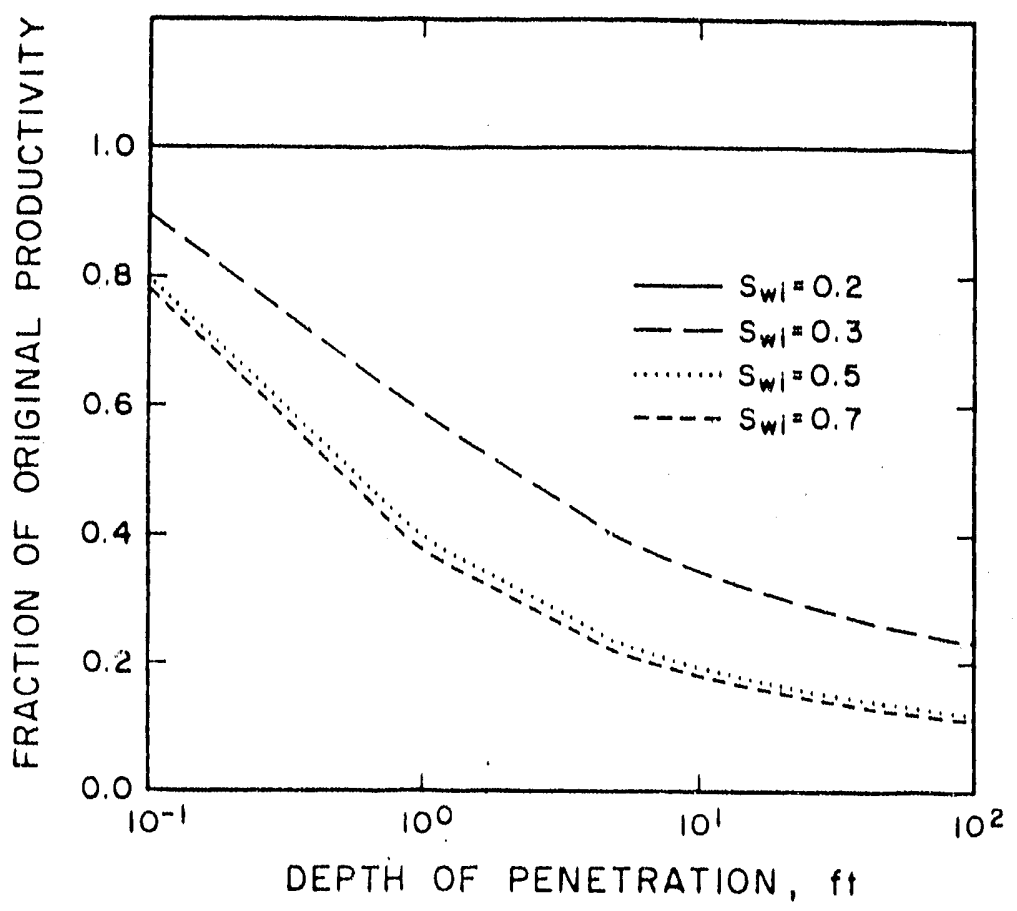

Fig. 53b. Productivity Retained after Gel Treatment with Hysteresis of Relative Permeability Curves $\left(F_{\mathrm{rww}}=10, \mathrm{~F}_{\mathrm{rro}}=1, \mathrm{n}_{\mathrm{w}}=6, \mathrm{n}_{\mathrm{o}}=3\right.$, radial flow $)$. 


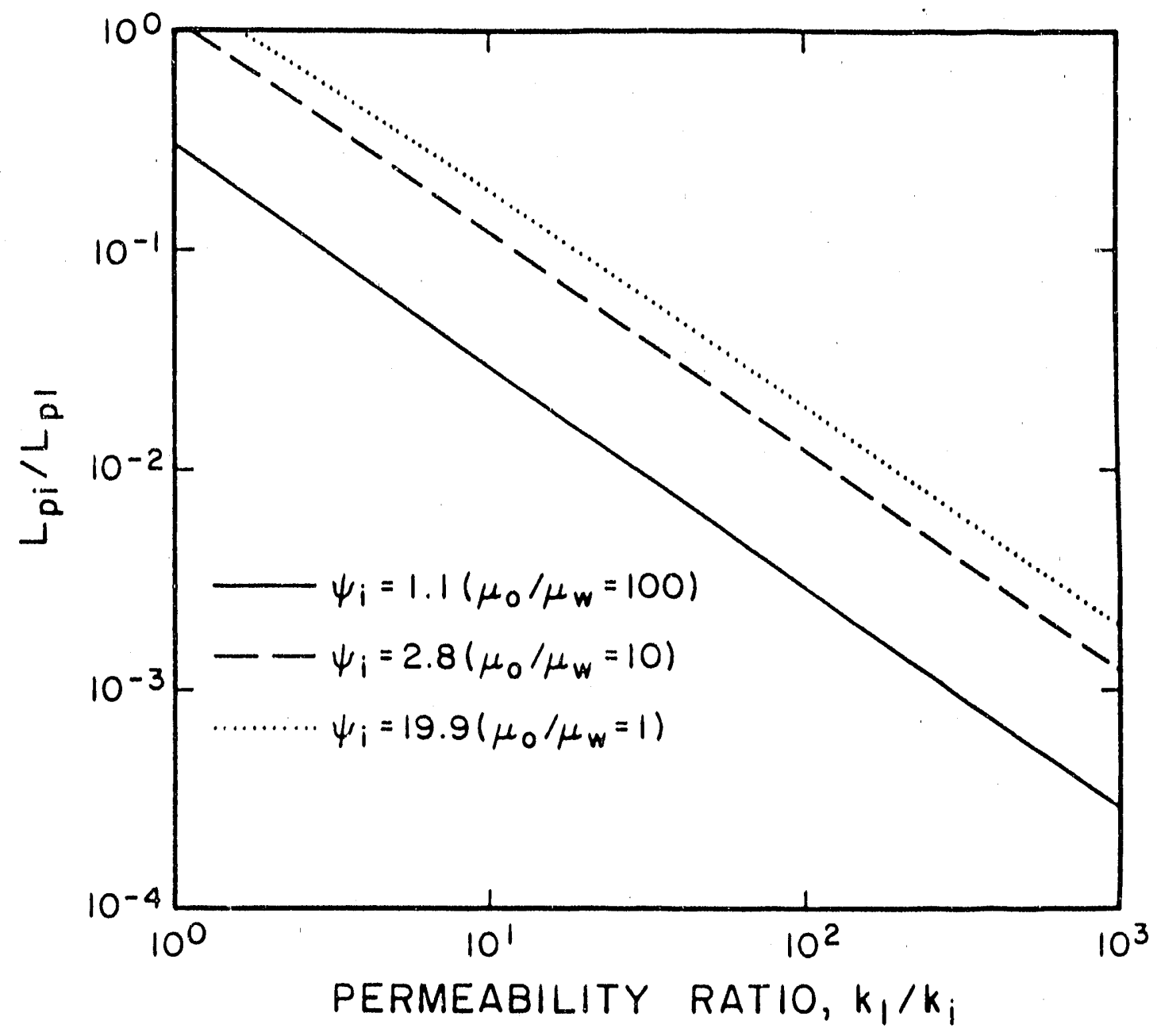

Fig. 54. Effect of $\Psi_{1}$ Variations $\left(F_{r}=1\right)$ on Degree of Penetration in Vertically Fractured Zones. 
For the case of constant pressure drop, the fractions of the original water and oil productivities af ter treatment in vertically fractured reservoirs are:

$$
\begin{aligned}
& \frac{q_{w i}}{q_{w o i}}=\frac{\left(\Psi_{i}+1\right) L_{p m}}{\frac{k_{r m i}}{k_{r m+i}} L_{p i}+\left(L_{p m}-L_{p i}\right)+\Psi_{i} L_{p m}} \\
& \frac{q_{o i}}{q_{\rho o i}}=\frac{\left(\Psi_{i}+1\right) L_{p m}}{\frac{k_{r a 1}}{k_{r o t i}} L_{p i}+\left(L_{p m}-L_{p i}\right)+\Psi_{i} L_{p m}}
\end{aligned}
$$

As in the cases of unfractured wells, the material balance dictates that the fraction of productivity loss of oil for a given zone after treatment is the same as that of water after treatment.

As shown in Fig. 55a, the productivity loss af ter treatment in a vertically fractured reservoir is relatively insensitive to invasion by the gelant (up to $\sim 10 \mathrm{ft}$ ). Figure $55 \mathrm{a}$ also shows that the productivity loss increases with increasing water saturation. A comparison of Fif.. 53a and Fig. 55a discloses that under similar circumstances the productivity loss after treatment in a vertically fractured reservoir is less than that in an unfractured reservoir. A similar comparison between Fig. $53 \mathrm{~b}$ and $\mathrm{Fig}$. 55b reveals that, even by taking hysteresis into account, the decline in productivity (that results from the invasion of gelants) is far less dramatic in vertically fractured wells than in unfractured wells.

\section{Effects of Crossflow}

In the analysis presented to this point, no crossflow occurs between adjacent layers. If crossflow can occur between layers or flow paths in a reservoir, viscous gelants will penetrate into low-permeability layers to a greater extent than if crossflow is not possible. In fact under some circumstances (if the gelant/water mobility ratio is less than the permeability contrast between adjacent layers), the depth of penetration of gelant in a low-permeability layer can be the same as that in an adjacent high-permeability layer. ${ }^{2}$ Thus, if crossflow can occur, viscous gelants will damage oil-productive zones to a greater extent than if crossflow is not possible. Discussion presented in previous sections regarding cases without crossflow can still serve as important guidelines in designing polymer gel treatments in reservoirs with vertical communication between layers.

\section{Control of Water Coning}

Field experience in the Arbuckle formation in western Kansas ${ }^{2}$ demonstrates that polymer gels can be very effective in treating production wells with water coning problems.

Water coning is a rate-sensitive phenomenon. The rise of a water table under a partially penetrated oil well is caused by the motion of oil above it. Hence, the maximum cone height at a given oil production rate is dictated by the balance between the hydrostatic head of the elevated water column and the upward pressure gradients associated with the oil flow. 


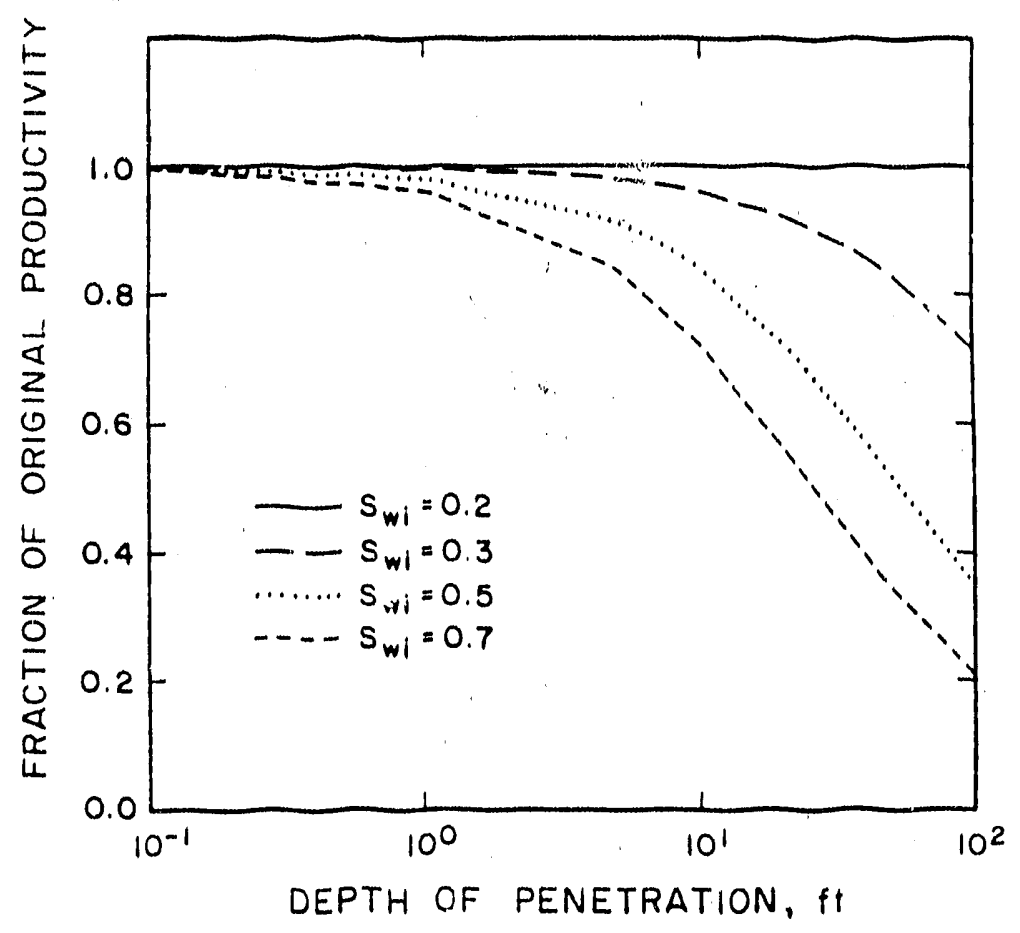

Fig. 55a. Effects of Degree of Penetration and $S_{w i}$ on Productivity Loss after Gel Treatment $\left(F_{\text {rww }}=\right.$ $10, F_{r o}=1, n_{w}=2, n_{0}=3$ ) in Vertically Fractured Zones.

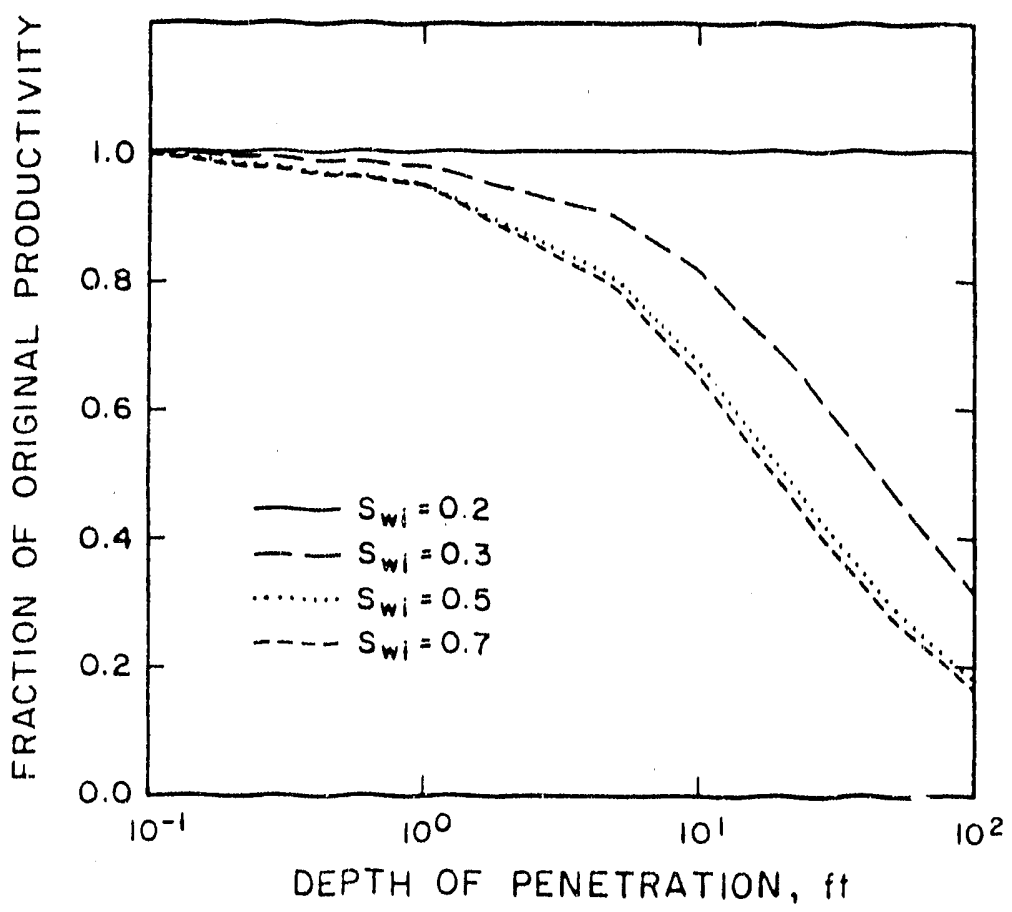

Fig. 55b. Productivity Retained after Gel Treatment with Hysteresis of Relative Permeability Curves $\left(F_{\mathrm{rw}}=10, F_{\mathrm{rro}}=1, \mathrm{n}_{\mathrm{w}}=6, \mathrm{n}_{\mathrm{o}}=3\right)$ in Vertically Fractured Zones. 
Based upon the free-surface concept, the following equation (proposed by Muskat et al.) ) $^{109}$ is used to solve for the maximum production rate at which a well can maintain water-free production.

$$
q_{0}=\frac{\pi k_{m} g\left(\rho_{w}-\rho_{o}\right)\left(h_{e}^{2}-h_{w^{2}}^{2}\right)}{\mu_{0} \ln \left(r_{e} / r_{0}\right)}
$$

where $h_{e}$ is the thickness of the oil zone and $h_{w}$ is the depth of well penetration. The cone shape at the critical production rate is defined by the following equation. ${ }^{110}$

$$
Z(r)=h_{e}-\sqrt{h_{e}^{2}-\left(h_{e}^{2}-h_{w}^{2}\right) \frac{\ln \left(r_{e} / r\right)}{\ln \left(r_{e} / r_{0}\right)}}
$$

where $Z(r)$ is the cone height at a certain radius $r$. Karp et al. ${ }^{110}$ suggested that water coning can be controlled or completely suppressed by means of horizontal barriers. The placement of horizontal barriers increases the ef fective wellbore radius. According to Eq. 55, this would increase the critical rate for water-free production.

Gelant can be injected into a formation to serve as a horizontal barrier. If polymer gels can reduce relative permeability to water without reaucing oil permeability, then oil productivity can be maintained while suppressing excess water production.

In some reservoirs, water from the underlying water zone migrates through a fracture system into oil-producing wells. The permeability of a fracture is much greater than the permeability of the adjacent formation rack. Thus, the behavior of fluid flow from the underly'ng water zone through a fracture into a production well can be approximated by a 2-D linear flow model rather than by a $3-D$ radial flow model. The critical rate for water-free production in a 2-D linear system can be estimated using the following equation. ${ }^{111}$

$$
q_{o}=\frac{k_{f} g W\left(\rho_{w}-\rho_{0}\right)\left(h_{e}^{i}-h_{w}^{2}\right)}{2 \mu_{o}\left(L-x_{0}\right)}
$$

where $W$ is the width of the fracture, $L$ is the length of the fracture, $x$ is the distance of gelant penetration into the fracture, and $x_{0}$ is the wellbore radius. The equation presented below defines the cone shape at the critical production rate in the 2-D model. This equation was derived by following the same procedure used by Karp et al. ${ }^{110}$ in deriving Eq. 56.

$$
Z(x)=h_{e}-\sqrt{h_{e}^{2}-\left(h_{e}^{2}-h_{w}^{2}\right) \frac{(L-x)}{\left(L-x_{0}\right)}}
$$

During the treatment process, gelants flow preferentially into the fracture because of the enormous permeability contrast between the fracture and the formation rock. By filling up the fracture with a polymer gel, we essentially convert the 2-D linear flow geometry into a 3-D radial flow genmetry. Dividing the critical rate given in Eq. 55 by that in Eq. 57 provides a means of comparing the severity of coning problems in a fractured well vs. that in an unfractured well. 
For the given set of parameters shown in the following example calculation, the critical production rate is found to be about 2 orders of magnitude higher in an unf ractured well than in a fractured well.

$$
\begin{aligned}
\frac{q_{o(3-D)}}{q_{o(2-D)}} & =\frac{2 \pi\left(L-x_{0}\right) k_{m}}{W \ln \left(r_{\mathrm{e}} / r_{0}\right) k_{f}}=\frac{2 \pi(372-0.5) 1}{(0.01 / 12) \ln (372 / 0.5) 1000} \\
& \approx 400
\end{aligned}
$$

Thus, some of tha successes observed in field applications can be attributed to the significant increase in critical production rate resulting from the flow-geometry conversion after gel treatment.

However, under other circumstances, the effect of this flow-geometry conversion may be less. If, for example, we change the fracture length $(\mathrm{L})$ in the previous example to $50 \mathrm{ft}$, then the increase in critic l production rate will be about one order of magnitude less than that shown above. Other parameters, such as the permeability contrast between the fracture and the adjacent formatic $n$ rock, the fracture width, and the drainage radius, also have an important influence on the critical production rate.

A small amount of gelant still penetrates into the rock matrix, forming a thin layer of gel around the wellbore. However, damage to oil productivity in the well can be minimized if polymer gels reduce the relative permeability to water without reducing that to oil.

In the examples presented in this report, we have assumed that gel will not affect the relative permeability to oil. If gel does reduce oil permeability, then some of our calculations will underestimate the loss of oil productivity. Thus, determination of permeability reductions for both oil and water are very important when planning field applications of gels in production wells. The equations and analyses in this report are general and will accommodate permeability reductions to oil as well as to water.

\section{Conclusions}

1. If zones are not isolated during gel placement in production wells, gelants can penetrate to a significant degree into all open zones-not just those with high water saturations.

2. For gels that reduce permeability to water more than to oil, induced changes in the relative permeability curves near-wellbore wil! not necessarily enhance oil recovery from a particular zone. Depending on the steady-state fractional flows of fluid outside of the gel-treated region, oil production could be impaired even though the gel reduces water permeability without affecting oil permeability. The principal advantage of the disproportionate reduction of the water and oil relative permeabilities is in reducing the need for zone isolation during gel placement. Realizing this advantage generally requires high fractional oil flow from oilproductive zones.

3. Under similar circumstances, the productivity loss after treatment in vertically fractured wells is expected to be less than that in unfractured wells.

4. An explanation is provided for why some successful applications of gels have occurred in fractured wells that are produced by bottom-water drive. With the right properties, gels could significantly increase the critical rate for water coning. 


\section{NOMENCLATURE}

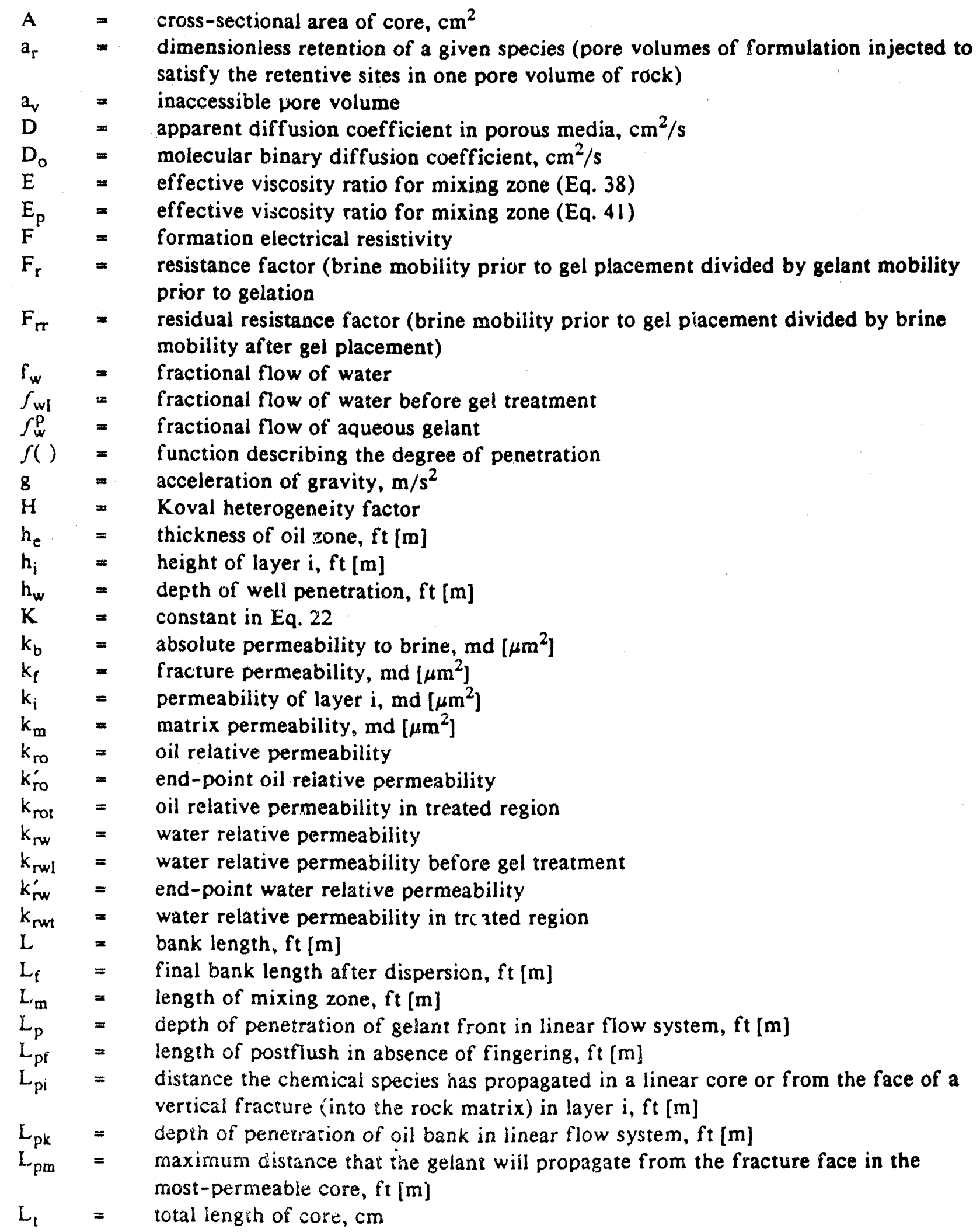




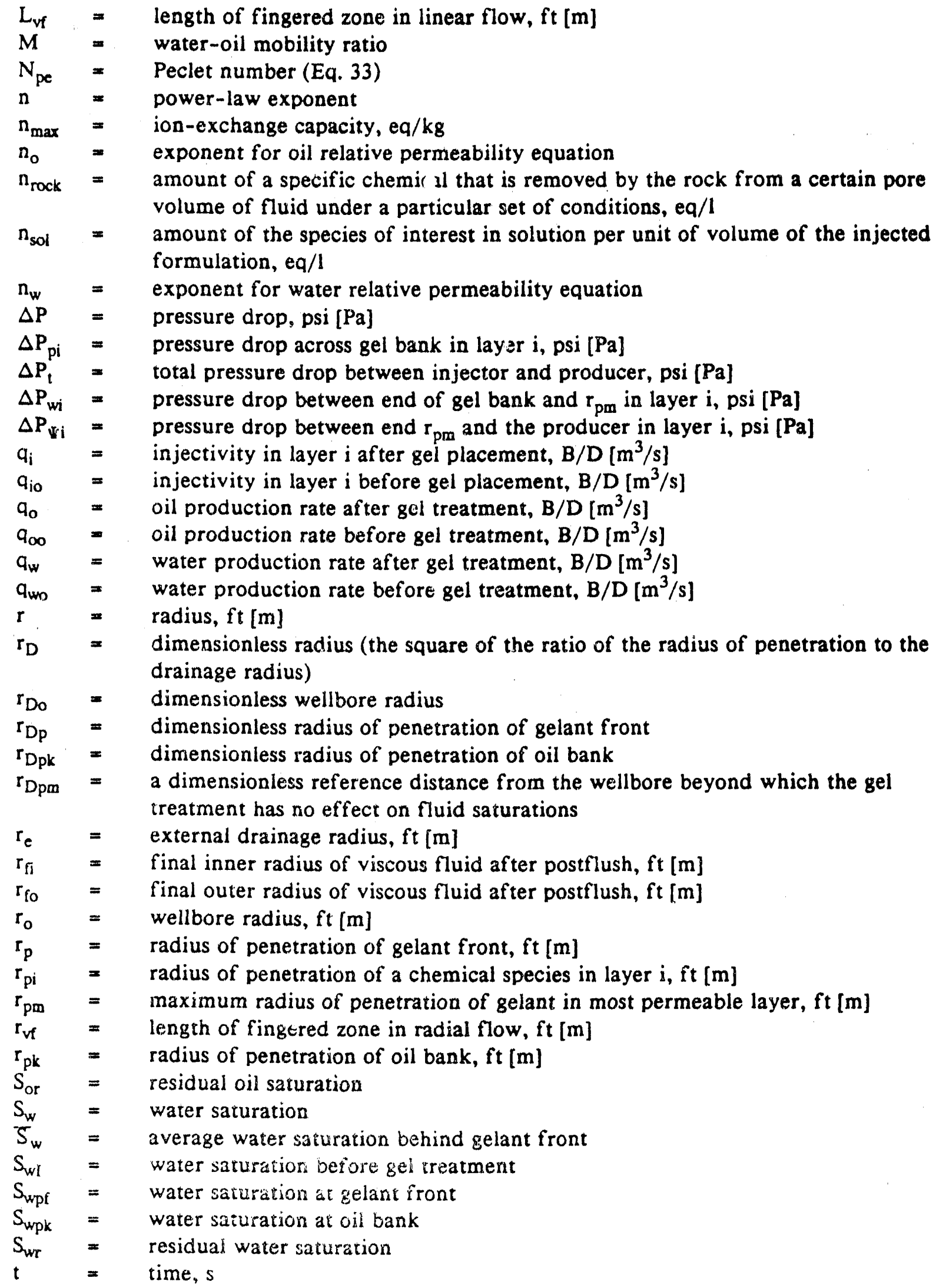




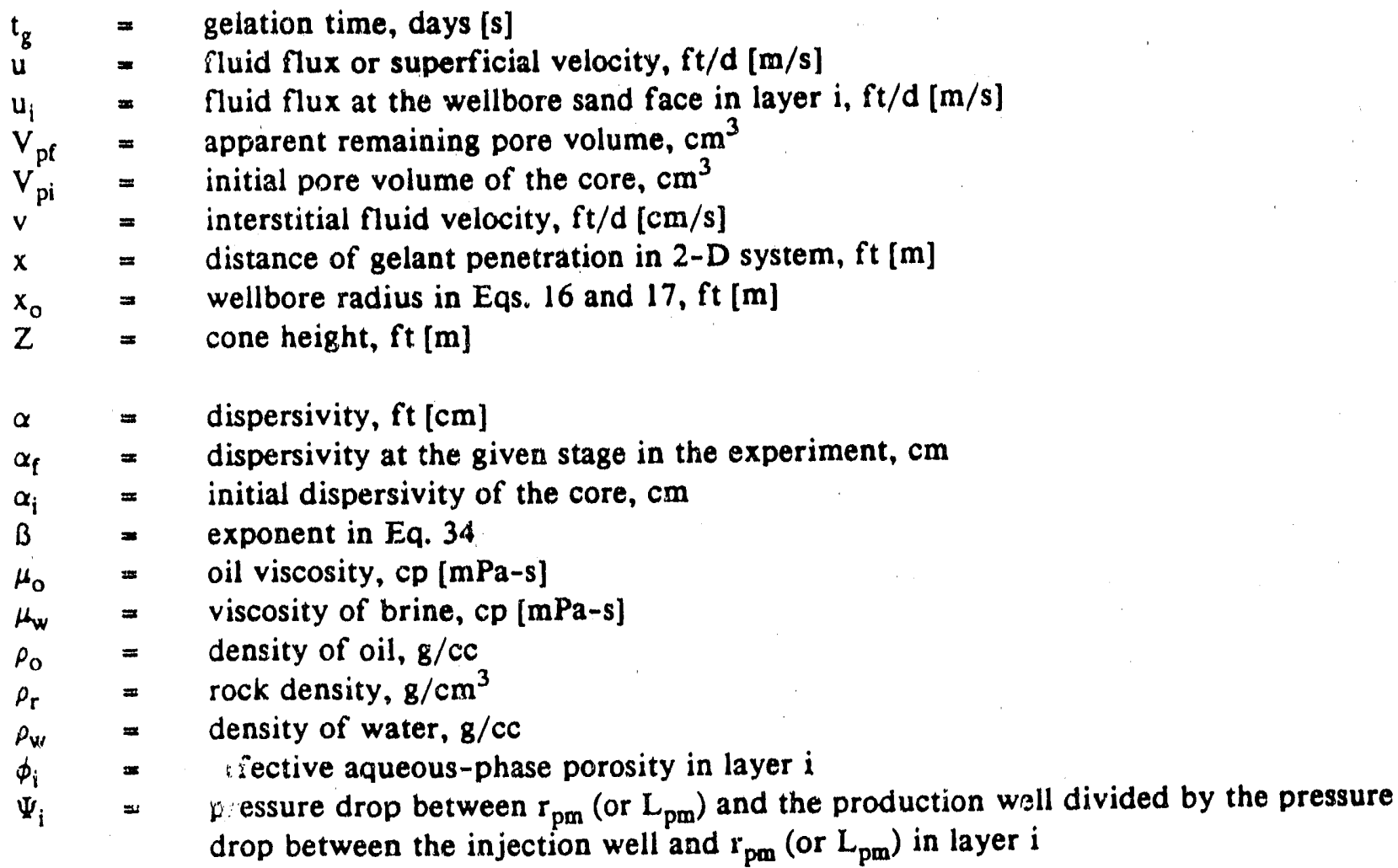




\section{REFERENCES}

1. DuBois, B.M.: "North Stanley Polymer Demonstration Project, Third Annual and Final Report," Report BETC/RI-78/19, U.S. Dept. of Energy, Washington, D.C. (Nov. 1978).

2. Hessert, J.E. and Fleming, P.D., III: "Gelled Polymer Technology for Control of Water in Injection and Production Wells," Proc, 1979 Third Tertiary Oil Recovery Conference, Wichita, KS (Apr. 25-26) 58-70.

3. Woods, P. et al:: "In-Situ Polymerization Controls $\mathrm{CO}_{2}$ /Water Channeling at Lick Creek," SPE/DOE paper 14958 presented at the 1986 SPE/DOE Symposium on Enhanced Oil Recovery held in Tulsa, OK (Apr. 20-23).

4. Wagner, O.R., Weisrock, W.P., and Patel, C.: "Field Application of Lignosulfonate Gels to Reduce Channeling, South Swan Hills Miscible Unit, Alberta, Canada," SPE paper 15547 presented at the 1986 SPE Annual Technical Conference and Exhibition held in New Orleans, LA (Oct. 5-8).

5. Leonard, J.: "Increased Rate of EOR Brightens Outlook," Oil \& Gas J. (Apr. 14, 1986) 88-94.

6. Schurz, G. et al.: "Polymer Augmented Waterflooding and Control of Reservoir Heterogeneity," Proceedings of the 1989 Petroleum Technology Into the Second Century Symposium held in Socorro, NM (Oct. 16-19).

7. "Near-Wellbore Technology," Phillips brochure 1024-87LT (1987).

8. Willhite, G.P., Green, D.W., Young, T.S., Thies, J.L., Michnick, M.J., Vossoughi, S., and Terry, R.E.: "Evaluation of Methods of Reducing Permeability in Porous Media by In-Situ Polymer Treatments," Final Report to DOE, Contract No. AS19-80BC10354, National Technical Information Service DOE/BC/10354-16 (Feb. 1986).

9. Sydansk, R.D.: "A New Conformance Improvement Treatment Chromium (III) Gel Technology," paper SPE/DOE 17329 presented at the 1988 SPE/DOE Enhanced Oil Recovery Symposium, Tulsa, OK (Apr. 17-20).

10. Terry, R.E. et al.: "Correlation of Gelation Times for Polymer Solutions Used as Sweep Improvement Agents," SPEJ (Apr. 1981) 229-235.

11. Jordan, D.S. et al:: "The Effect of Temperature on Gelation Time for PolyacrylamideChromium (III) Systern," SPEJ (Aug. 1982) 463-471.

12. Batycky, J.P., Maini, B.B., and Milosz, G.: "A Study of the Application of Polymeric Gels in Porous Media," paper SPE 10620 presented at the 1982 SPE International Symposium on Oilfield and Geothermal Chemistry, Dallas, TX (Jan. 25-27).

13. DiGiacomo, P.M. and Schramm, C.M.: "Mechanism of Polyacrylamids Gel Syneresis Determined by C-13 NMR," paper SPE 11787 presented at the 1983 SPE International Symposium on Oilfield and Geothermal Chemistry, Denver, CO (Jun. 1-3).

14. Prud'homme, R.K. et al:: "Rheological Monitoring of the Formation of Polyacrylamide/ $\mathrm{Cr}^{+3}$ Gels," SPEJ (Oct. 1983) 804-808. 
15. Aslam, S., Vossoughi, S., and Willhite, G.P.: "Viscometric Measurement of Chromium(III)Polyacrylamide Gels by Weissenberg Rheogoniometer," paper SPE/DOE 12639 presented at the 1984 SPE/DOE Fourth Symposium on Enhanced Oil Recovery, Tulsa, OK (Apr. 15-18).

16. Prud'homme, R.K. and Uhl, J.T.: "Kinetics of Polymer/Metal-Iou Gelation," paper SPE/DOE 12640 presented at the 1984 SPE/DOE Fourth Symposium on Enhanced Oil Recovery, Tulsa, OK (Apr. 15-18).

17. Southard, M.Z., Green, D.W., and Willhite, G.P.: "Kinetics of the Chromium(VI)/Thiourea Reaction in the Presence of Polyacrylamide, " paper SPE 12715 presented at the 1984 SPE/DOE Fourth Symposium on Enhanced Oil Recovery, Tulsa, OK (Apr. 15-18).

18. Ghazali, H.A. and Willhite, G.P.: "Permeability Modifications Using Aluminum Citrate/Polymer Treatunents: Mechanisms of Permeability Reduction in Sandpacks, " paper SPE 13583 presented at the 1985 SPE International Symposium on Oilfield and Geothermal Chemistry, Phoenix, AZ (Apr. 9-11).

19. Hubbard, S., Roberts, L.J., and Sorbie, K.S.: "Experimental and Theoretical Investigation of Time-Setting Polymer Gels in Porous Media," paper SPE/DOE 14959 presented at the 1986 SPE/DOE Fifth Symposium on Enhanced Oil Recovery, Tulsa, OK (Apr. 20-23).

20. Hedges, J.H.: "Chromium-Polyacrylamide Gelation and Quality Control for Field Applications," paper SPE 15790, unsolicited (1986).

21. Huang, C.G., Green, D.W., and Willhite, G.P.: "An Experimental Study of the In-Situ Gelation of Chromium(III)/Polyacrylamide Polymer in Porous Media," SPE Reservoir Engineering (Nov. 1986) 583-592.

22. Aslam, S., Vossoughi, S., and Willhite, G.P.: "Viscometric Measurement of Chromium (III)Polyacrylamide Gels," Chem. Eng. Commun. 48 (1986) 287-301.

23. Nanda, S.K. et al.: "Characterization of Polyacrylamide-Cr ${ }^{+6}$ Gels Used for Reducing Water/Oil Ratio," paper SPE 16253 presented at the 1987 SPE International Symposium on Oilfield and Geothermal Chemistry, San Antonio, TX (Feb. 4-6).

24. Seright, R.S.: "Placement of Gels to Modify Injection Profiles," paper SPE/DOE 17332 presented at the 1988 SPE/DOE Enhanced Oil Recovery Symposium, Tulsa, OK (Apr. 17-20).

25. Seright, R.S.: "Effect of Rheology on Gel Placement," paper SPE 18502 presented at the 1989 SPE International Symposium on Oilfield Chemistry, Houston, TX (Feb. 8-10).

26. Seright, R.S.: "Impact of Dispersion on Gel Placement for Profile Control, " paper SPE 20127 presented at the 1990 SPE Permian Basin Oil \& Gas R.ecovery Conference, Midland, TX (Mar. 8-9).

27. Liang, J., Lee, R.L., and Seright, R.S.: "Placement of Gels in Production Wells," paper SPE/DOE 20211 presented at the 1990 SPE/DOE Enhanced Oil Recovery Symposium, Tulsa, OK (Apr. 22-25).

28. Campbell, B.T. and Orr, F.M., Jr.: "Flow Visualization for $\mathrm{CO}_{2} /$ Crude-Oil Displacements," SPEJ (rizt. 1985) 665-678. 
29. Martin, F.D. and Kovarik, F.S.: "Chemical Gels for Diverting $\mathrm{CO}_{2}:$ Baseline Experiments," paper SPE 16728 presented at the 198\%' Technical Conference and Exhibition, Dallas, TX (Sept. 27-30).

30. Martin, F.D., Kovarik, F.S., Charig, P.W., and Phillips, J.C.: "Gels for $\mathrm{CO}_{2}$ Profile Modification," paper SPE/DOE 17330 presented at the 1988 SPE/DOE Symposium on Enhanced Oil Recovery, Tulsa, OK (Apr. 17-20).

31. Chang, P.W., Goldman, I.M., and Stingley, K.J.: "Laboratory Studies and Field Evaluation of a New Gelant for High-Temperature Profile Modification," paper SPE 14235 presented at the 1985 SPE Annual Technical Conference and Exhibition, Las Vegas (Sep. 22-25).

32. Chang, P.W. et al.: "Enhanced Hydrocarbon Recovery by Permeability Modification with Phenolic Gels," U.S. Patent No. 4,708,974 (1987).

33. A very, M.R., Burkholder, L.A., and Gruenenfelder, M.A.: "Use of Crosslinked Xanthan Gels in Actual Profile Modification Field Projects," paper SPE 14114 presented at the 1986 SPE International Meeting on Petroleum Engineering, Beijing (Mar. 17-20).

34. Sydanik, R.D. and Smith, T.B.: "Field Testing of a New Conformance - Improvement Treatment - Chromium (III) Gel Technology," paper SPE/DOE 17383 presented at the 1988 SPE/DOE Enhanced Oil Recovery Symposium held in Tulsa, OK (Apr. 17-20).

35. Morrocico, M.L.: "Gel for Retarding Water Flow," U.S. Patent No. 4,498,540 (1985).

36. Jurinak, J.J., Summers, L.E., and Bennett, K.E.: "Oilfield Application of Colloidal Silica Gel," paper SPE 18505 presented at the 1989 SPE International Symposium on Oilfield Chemistry, Houston, TX (Feb. 8-10).

37. Eggert Jr., R.W., Willhite, G.P., and Green, D.W.: "Experimental Measurment of the Persistence of Permeability Reduction in Porous Media Treated With Xanthan $/ \mathrm{Cr}$ (III) Gel Systems," paper SPE 19630 presented at the 1989 Annual Technical Conference and Exhibition, San Antonio, TX (Oct. 8-11).

38. Bunge, A.L. and Radke, C.J.: "Migration of Alkaline Pulses in Reservoir Sands," SPEJ (Dec. 1982) 998-1012.

39. Crolet, J.L. and Bonis, M.R.: "pH Measurements under High Pressures of $\mathrm{CO}_{2}$ and $\mathrm{H}_{2} \mathrm{~S}$," Materials Performance (May, 1984) 35-42.

40. Vela, S., Peaceman, D.W., and Sandvik, E.I.: "Evaluation of Polymer Flooding in a Layered Reservoir With Crossflow, Retention, and Degradation," SPEJ (Apr. 1976) 82-96.

41. Walsh, M.P. et al.: "Chemical Interactions of Aluminum-Citrate Solutions with Formation Minerals," SPE paper 11799 presented at the 1983 SPE International Symposium on Oilfield Chemistry, Denver, CO (Jun. 1-3).

42. Garver, F.J., Sharma, M.M., and Pope, G.A.: "The Competition for Chromium Between Xanthan Biopolymer and Resident Clays in Sandstones," SPE paper 19632 presented at the 1989 SPE Annual Technical Conference and Exhibition, San Antonio, TX (Oct. 8-11). 
43. Parmeswar, R. and Willhite, G.P.: "A Study of the Reduction of Brine Permeability in berea Sandstone with the Aluminum Citrate Process," SPE Reservoir Eng. (Aug. 1988) 959-966.

44. Morrison, R.T. and Boyd, R.N.: Organic Chemistry, fourth edition, Allyn and Bacon, Boston, MA (1983) 978-979.

45. Navratil, M., Sovak, M., and Mitchell, M.S.: "Formation Blocking Agents: Applicability in Water- and Steamflooding," SPE paper 12006 presented at the 1983 Annual Technical Conference and Exhibition, San Francisco, CA (Oct. 5-8).

46. Nagra, S.S. et al.: "Stability of Waterflood Diverting Agents at Elevated Temperatures in Reservoir Brines," SPE paper 15548 presented at the 1986 Annual Technical Conference and Exhibition, New Orleans, LA (Oct. 5-8).

47. Mohnot, S.M., Bae, J.H., and Foley, W.L.: "A Study of Mineral/Alkali Reactions," SPE Reservoir Eng. (Nov. 1987) 653-663.

48. Sydansk, R.D.: "Elevated-Temperature Caustic/Sandstone Interaction: Implications for Improving Oil Recovery," SPEJ (Aug. 1982) 453-462.

49. Mohriot, S.M. and Bae, J.H.: "A Study of Mineral/Alkali Reactions--Part 2," SPE Reservoir Eng. (Aug. 1989) 381-390.

50. Kia, S.F., Fogler, H.S., and Reed, M.G.: "Effect of pH on Colloidally Induced Fines Migration," J. Colloid \& Interfac. Sci. 118(1) (1987) 158-168.

51. Reppert, T.R. et al.: "Second Ripley Surfactant Flood Pilot Test," paper SPE/DOE 20219 presented at the 1990 SPE/DOE Enhanced Oil Recovery Symposium, Tuisa, OK (Apr. 22-25).

52. Perkins, T.K. and Johnston, O.C.: "A Review of Diffusion and Dispersion in Porous Media," SPEJ (Mar. 1963) 70-84.

53. Coats, K.H. and Smith, B.D.: "Dead-End Pore Volume and Dispersion in Porous Media," SPEJ (Mar. 1964) 73-84.

54. Jensen, J.A, and Radke, C.J.: "Chromatographic Transport of Alkaline Buffers Through Reservoir Rock," SPE Reservoir Eng. (Aug. 1988) 849-856.

55. Lorenz, P.B. and Peru, D.A.: "Guidelines help select reservoirs for $\mathrm{NaHCO}_{3} \mathrm{EOR}$, , Oil \& Gas J. (Sep. 11, 1989) 53-57.

56. Needham, R.B., Threlkeld, C.B., and Gall, J.W.: "Control of Water Mobility Using Polymers and Multivalent Cations," paper SPE 4747 presented at the 1974 SPE-AIME Improved Oil Recovery Symposium, Tulsa, OK. (April).

57. Sandiford, B.B. and Graham, G.A.: "Injection of Polymer Solutions in Producing Wells," AIChE Symposium Series, Vol. 69, 127 (1973) 38.

58. Schneider, F.N. and Owens, W.W.: "Steady-State Measurements of Relative Permeability for Polymer/Oil Systems," SPE'J (Feb. 1982) 79. 
59. Sloat, B.: "Increasing Oil Recovery by Chemical Control of Producing Water-Oil Ratios," paper SPE 5341 presented at the 1975 SPE-AIME Rocky Mountain Regional Meeting, Denver (April).

60. Zaitoun, A. and Kohler, N.: "Two-Phase Flow Through Porous Media: Effect of an Adsorbed Polymer Layer," paper SPE 18085 presented at the 1988 Annual Technical Conference and Exhibition, Houston, TX (Oct. 2-5).

61. Bird, R.B., Stewart, W.E., and Lightfoot, E.N.: Transport Phenomena, John Wiley, New York (1960) 197-207.

62. Christopher, R.H. and Middleman, S.: "Power-Law Flow Through a Packed Tube," Ind. Eng. Chem. Fundam., 4, No. 4 (Nov. 1965) 422-426.

63. Gogarty, W.B., Levy, G.L., and Fox, V.G.: "Viscoelastic Effects in Polymer Flow Through Porous Media," paper SPE 4025 presented at the 1972 SPE Annual Meeting, San Antonio, TX (Oct. 8-11).

64. Teeuw, D. and Hesselink, F.T.: "Power-Law Flow and Hydrodynamic Behavior of Biopolymer Solutions in Porous Media," paper SPE 8982 presented at the 1980 SPE Fifth International Symposium on Oilfield and Geothermal Chemistry, Stanford, CA (May 28-30).

65. Chang, P.W. et al.: "Selective Emplacement of Xanthan/Cr(III) Gels in Porous Media," SPE paper 17589 presented at the 1988 SPE International Meeting on Petroleum Engineering, Tianjin, China (Nov. 1-4).

66. Hejri, S., Green, D.W., and Willhite, G.P.: "In-Situ Gelation of a Xanthan/Cr(III) Gel System in Porous Media," paper SPE 19634 presented at the 1989 SPE Annual Technical Conference and Exhibition, Sán Antonio, TX (Oct. 8-11).

67. Jousset, F. et al:: "Effect of High Shear Rate on In-Situ Gelation of a Xanthan/Cr(III) System," paper SPE/DOE 20213 presented at the 1990 SPE/DOE Symposium on Enhanced Oil Recovery, Tulsa, OK (Apr. 22-25).

68. Todd, B.J., Green, D.W., and Willhite, G.P.: "A Mathematical Model of In-Situ Gelation of

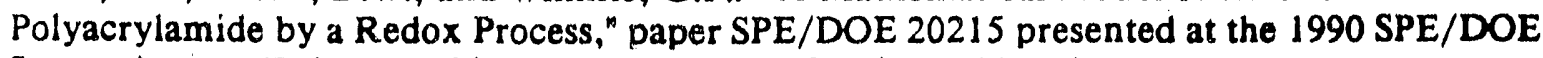
Symposium on Enhanced Oil Recovery, Tulsa, OK (Apr. 22-25),

69. Zapata, V.J. and Lake, L.W.: "A Theoretical Analysis of Viscous Cross flow," paper SPE 10111 presented at the 1981 SPE Annual Technical Conference and Exhibition, San Antonio, TX (Oct. 5-7).

70. Zapata, V.J.: "A Theoretical Analysis of Viscous Crossflow," PhD Dissertation, The University of Texas (1981).

71. Young, T.S., Willhite, G.P., and Green, D.W.: "Study of Intra-Molecular Crosslinking of Polyacrylamide in $\mathrm{Cr}$ (III)-Polyacrylamide Gelation by Size-Exclusion Chromatography, LowAngle Laser Light Scattering, and Viscometry," Water-Soluble Polymers for Petroleum Recovery, G.A. Stahl and D.N. Schulz (eds.), Plenum Press, New York (1988) 329-342.

72. Erdey-Gruz, T.: Transpors Phenomena in Aqueous Solutions, John Wiley, New York (1974) $151-163$. 
73. Southwick, J.G., Jamieson, A.M., and Blackwell, J.: "Conformation of Xanthan Dissolved in Aqueous Urea and Sodium Chloride Solutions," Carbohydrate Research (1982) 99, 117-127.

74. Arya, A. et al.: "Dispersion and Reservoir Heterogeneity," SPERE (Feb. 1988) 139-148.

75. Kolodziej, E.J.: "Transport Mechanisms of Xanthan Biopolymer Solutions in Porous Media," paper SPE 18090 presented at the 1988 SPE Annual Technical Conference and Exhibition, Houston, TX (Oct. 2-5).

76. Heller, J.P.: "1 he Interpretation of Model Experiments for the Displacement of Fluids Through Porous Media," AIChE J. 9, No. 4 (1963) 452-459.

77. Perkins, T.K., Johnston, O.C., and Hoffman, R.N.: "Mechanics of Viscous Fingering in Miscible Systems," SPEJ (Dec. 1965) $301-317$.

78. Tang, D.H.E. and Peaceman, D.W.: "New Analytical and Numerical Solutions for the Radial Convection-Dispersion Problem," SPERE (Aug. 1987) 343-359.

79. Tang, D.H. and Babu, D.K.: "Analytical Solution of a Velocity Dependent Dispersion Problem," Water Resources Research 15 (Dec. 1979) 1471-78.

80. Koval, E.J.: "A Method for Predicting the Performance of Unstable Miscible Displacement in Heterogeneous Media," SPEJ (Jun. 1963) 145-154.

81. Claridge, E.L.: "Predicticn of Recovery in Unstable Miscible Flooding," SPEJ (Apr. 1972) 143-55.

82. Claridge, E.L.: "Control of Viscous Fingering in Enhanced Oil Recovery Processes: Effect of Heterogeneities," paper SPE 7662 (May 1978).

83. Todd, M.R. and Longstaff, W.J.: "The Development, Testing, and Application Of a Numerical Simulator for Predicting Miscible Flood Performance," JPT (Jul. 1972) 874-882.

84. Stalkup, F.h.: Miscible Displacement, SPE Monograph Vol. 8, SPE, New York (1983) 39-44.

85. Stoneberger, M.W. and Claridge, E.L.: "Graded-Viscosity-Bank Design With Pseudoplastic Fluids," SPERE (Nov. 1988) 1221-1232.

86. Clifford, P.J. and Sorbie, K.S.: "The Effects of Chemical Degradation on Polymer Flooding," paper SPE 13586 presented at the 1985 International Symposium on Oilfield and Geothermal Chemistry, Phoenix, AZ (Apr. 9-11).

87. Clifford, P.J.: "Simulation of Small Chemical Slug Behavior in Heterogeneous Reservoirs," paper SPE/DOE 17339 presented at the 1988 SPE/DOE Enhanced Oil Recovery Symposium, Tulsa, OK (Apr. 17-20).

88. Sorbie, K.S. and Clifford, P.J.: "The Simulation of Polymer Flow in Heterogeneous Porous Media," in Water-Soluble Polymers for Petroleum Recovery, G.A. Stahl and D.N. Schulz (eds.), Plenum Press, New York (1988) 69-99. 
89. Allen, E. and Boger, D.V.: "The Influence of Rheological Properties on Mobility Control in Polymer-Augmented Waterflooding," paper SPE 18097 presented at the 1988 SPE Annual Technical Conference and Exhibition, Houston, TX (Oct. 2-5).

90. Bragg, J.R.: Private communication (Nov. 1988).

91. Abdo, M.K. et al.: "Field Experience with Floodwater Diversion by Complexed Biopolymers," SPE/DOE paper 12642 presented at the 1984 SPE/DOE Fourth Symposium on Enhanced Oil Recovery, Tulsa, OK (Apr. 15-18).

92. Burkholder, L.A., Carruthers, M.S., and Rashan, J.M.: "Xanthan Gels for Injection Profile Control," presented at the 1984 Fifth Annual Advances in Petroleum Recovery \& Upgrading Technolc Jy (Jun. 14-15).

93. Sampath, K. et al.: "A New Biopolymer for High-Temperature Profile Control: Part II--Field Results," SPE paper 19867 presented at the 1989 Annual Technical Conference and Exhibition, San Antonio, TX (Oct. 8-11).

94. Dalrymple, D., Sutton, D., and Creel, P.: "Conformance Control in Oil Recovery," presented at the 1985 Southwest Petroleum Short Course, Lubbock, TX (April).

95. "Water Control Services," Halliburton Services brochure WCS-0244.

96. Hanlon, D.J., Fulton, S., and Beny, M.: "New Chemical and Mechanical Technology for Injection Profile Control, " presented at the 1987 Southwestern Petroleum Short Course, Lubbock, TX.

97. Doll, T.E.: "Polymer Mini-Injectivity Test: Shannon Reservoir, Naval Petroleum Reserve No. 3, Natrona County, WY," SPE paper 12925 presented at the 1984 Rocky Mountain Regional Meeting, Casper, WY (May 21-23).

98. Dovan, H.T. and Hutchins, R.D.: "Development of a New Aluminum-Polymer Gel System for Permeability Adjustment," SPE/DOE paper 12641 presented at the 1984 SPE/DOE Symposium on Enhanced Oil Recovery, Tulsa, OK (Apr. 15-18).

99. Howard, G.C. and Fast, C.R.: Hydraulic Fracturing, SPE, New York (1970) 149.

100. Koch, R.R. and McLaughlin, H.C.: "Fieid Performance of New Technique for Control of Water Production or Injection in Oil Recovery," paper SPE 2847 presented at the 1970 SPE Practical Aspects of Improved Recovery Techniques Meeting, Fort Worth, TX (March 8-10).

101. Avery, M.R. and Wells, T.A.: "Field Evaluation of a New Gelant for Water Control in Production Wells," paper SPE 18201 presented at the 1988 SPE Annual Technical Conference and Exhibition, Houston, TX (Oct. 2-5).

102. Olsen, E.H.: "Case History: Water Shutoff Treatment in the Phosphoria Formation, Hot Springs County, Wyoming," paper SPE 15163 presented at the 1986 SPE Rocky Mountain Regional Meeting, Billings, MT (May 19-21).

103. Lake, L.W.: Enhanced Oil Recovery, Prentice-Hall, Inc., Englewood Cliffs, New Jersey (1989) $58-62$. 
104, Pope, G.A.: "The Application of Fractional Flow Theory to Enhanced Oil Recovery," SPEJ (June 1980) 191-205.

105. Dake, L.P.: Fundamentals of Reservoir Engineering, Elsevier Scientific Publishing Co., New York (1982) 356-362.

106. Deppe, J.C.: "Injection Rates-The Ef fect of Mobility Ratio, Area Sweep, and Pattern," SPEJ (June 1961) 81-91.

107. Willhite G.P.: Waterflooding, SPE Textbook Series, Vol. 3 (1986).

108. Jones, S.C. and Roszelle, W.O.: "Graphical Techniques for Determining Relative Permeability From Displacement Experiments," JPT (May 1978) 807-817.

109. Muskat, M.: Physical Principles of Oil Production, McGraw-Hill Book Co., Inc., N.Y. (1949) 226.

110. Karp, J.C., Lowe, D.K., and Marusov, N.: "Horizontal Barriers for Controlling Water Coning," JPT (July 1962) 783-790.

111. Muskat, M.: Flow of Homogeneous Fluids Through Porous Media, McGraw-Hill Book Co., Inc., N.Y. (1946) 377. 
APPENDIX A

PROCEDURE FOR FABRICATION OF TWO-DIMENSIONAL GLASS MICROMODELS 


\section{APPENDIX A PROCEDURE FOR FABRICATION OF TWO-DIMENSIONAL GLASS MICROMODELS}

This report details techniques for preparing the two-dimensional glass micromodels that are being used in the project, "Fluid Diversion and Sweep Improvement with Chemical Gels in Oil Recovery Processes." McKellar and Wardlaw ${ }^{1}$, Chatzis ${ }^{2}$, and Campbell ${ }^{3}$ described the methods first used at the New Mexico Petroleum Recovery Research Center for fabricating two-dimensional glass micromociels. These micromodels were used in a number of flow-visualization studies. ${ }^{4-10}$ The techniques described here represent improvements on methods that were developed earlier. ${ }^{1-3}$

\section{SOLUTION PREPARATION}

The following are solutions needed for micromodel fabrication.

Mirror-backing strippers:

Alkyd backing stripper. (WARNING!!! Store only in glass. Use only in a glaws or steel pan.) $5 \%$ Formic acid $5 \%$ Acetic acid (Glacial)

$5 \%$ Phenol

85\% Methylene chloride

Epoxy backing stripper.

$50 \%$ Paint remover (e.g., Strippteze $\left.{ }^{\odot}\right)$

$50 \%$ Methylene chloride

Copper etcher:

$40 \%$ Nitric acid

$60 \%$ Distilled water

Copper stripper:

$60 \%$ Nitric acid

$40 \%$ Distilled water

Glass etcher: (WARNING!!! Store and use only with fluorine-proof materials.)

$110 \mathrm{~g}$ Ammonium hydrogen difluoride

$600 \mathrm{ml}$ Distilled water

$15 \mathrm{ml}$ Sulfuric acid

Bring up to 1 liter with distilled water

\section{SOLUTION HAZARDS}

All of the chemicais used in micromodel construction are harmful if swallowed, inhaled, or absorbed through skin. The materials are extremely destructive to tissue of tne mucous membranes and upper respiratory tract, eyes, and skin. Inhalation may be fatal as a result of spasm, inflammation and edema of the larynx and bronchi, chemical pneumonitis, or pulmonary edema. Symptoms of exposure may include burning sensation, cougning, wheezing, laryngitis, shortness of breath, headache, nausea, or vomiting. A fume hood should be used when working with these chemicals, and the worker should be properly clothed for protection from inhalation or physical contact with these chemicals. 


\section{Specific Chemical Hazards:}

Photo-resist and xylene $=>$ Skin irritant. Reproductive hazard.

Phenol $\Rightarrow$ Highly toxic. Possible teratogen. Vesicant. Reproductive hazard. Readily absorbed through skin. Light-sensitive. Combustible liquid.

Acetic acid $\Rightarrow$ Corrosive. Combustible liquid. Readily absorbed through skin.

Methylene chloride $\Rightarrow$ Possible carcinogen. Toxic. Neurologic hazard. Irritant.

Nitric acid $\Rightarrow$ Causes severe burns. Strong oxidizer.

Sulfuric acid $\Rightarrow$ Poison. Causes severe burns.

Ammonium hydrogen difluoride $=>$ Etches glass readily. Corrosive. Toxic. Hygroscopic.

\section{SOLUTION DISPOSAL}

Four separate hazardous wastes are generated using the techniques described in this report. Each should be stored separately and disposed using appropriate hazardous-waste procedures. The four wastes are as follows:

1. Waste mirror-backing stripper and stripped backing.

2. Waste photo-resist and xylene.

3. Waste nitric acid solutions. These should be neutralized with sodium bicarbonate, then allowed to evaporate and the solids collected. The solids are copper and silver salts.

4. Waste glass-etching solutions. These should first be mixed with enough calcium hydroxide to precipitate all of the fluorine. Then neutralize the solution with either sodium bicarbonate or sulfuric acid, depending on which way the $\mathrm{pH}$ needs to be adjusted. Let the solution settle for a few days, then pour the liquid down the sink and collect the calcium fluoride precipitate.

\section{FABRICATION STEPS}

\section{Step 1. Choice of Mirror and Glass Plate}

First, obtain plate-glass mirror and plate glass of the appropriate dimensions to accommodate the micromodel pattern. In our work, four micromodel patterns are in use. These are shown (actual size) in Figs. A-1 through A-4. Pore space is shown in black in these figures. Figure 1 is a block pattern $(0.2 \mathrm{~cm} \times 10 \mathrm{~cm})$ that we use to obtain inherent permeabilities of gels. Figure 2 is a very regular pattern with overall dimensions of $8 \mathrm{~cm} \times 12.8 \mathrm{~cm}$. The node diameters are $0.1 \mathrm{~cm}$, and the connections between nodes have widths of $0.025 \mathrm{~cm}$ and lengths of $0.2 \mathrm{~cm}$. The pattern shown in Fig. 3 represents a homogeneous rock structure $(7 \mathrm{~cm} \times 13.3 \mathrm{~cm})$. Finally, the pattern shown in Fig. $4(4.7$ $\mathrm{cm} \times 6.5 \mathrm{~cm}$ ) is a $10 \mathrm{X}$ magnification of a thin section from a San Andres carbonate rock. 
Fig. A-1. Pattern for Determining Gel Permeabilities

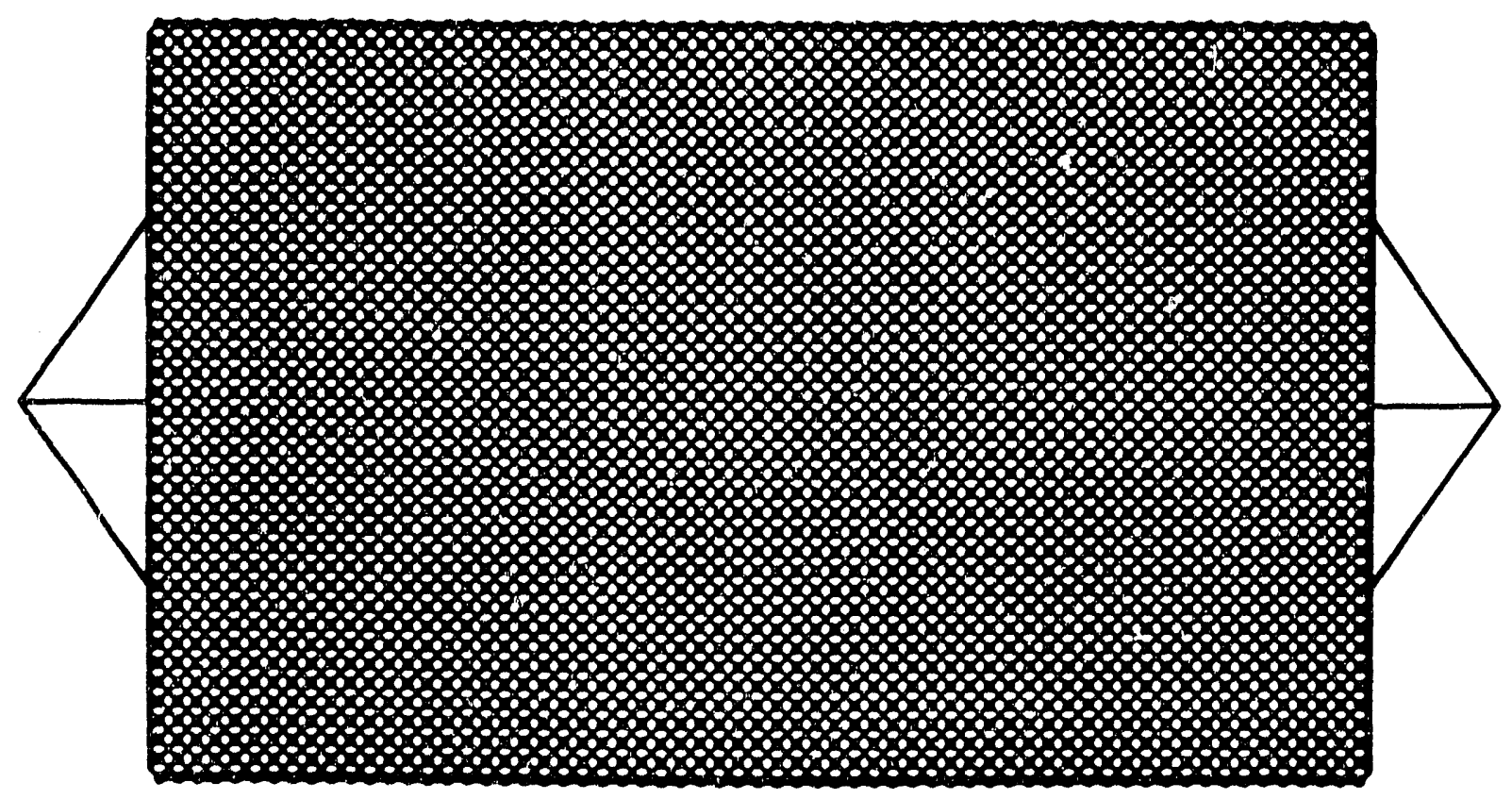

Fig. A-2. Regular Pattern 


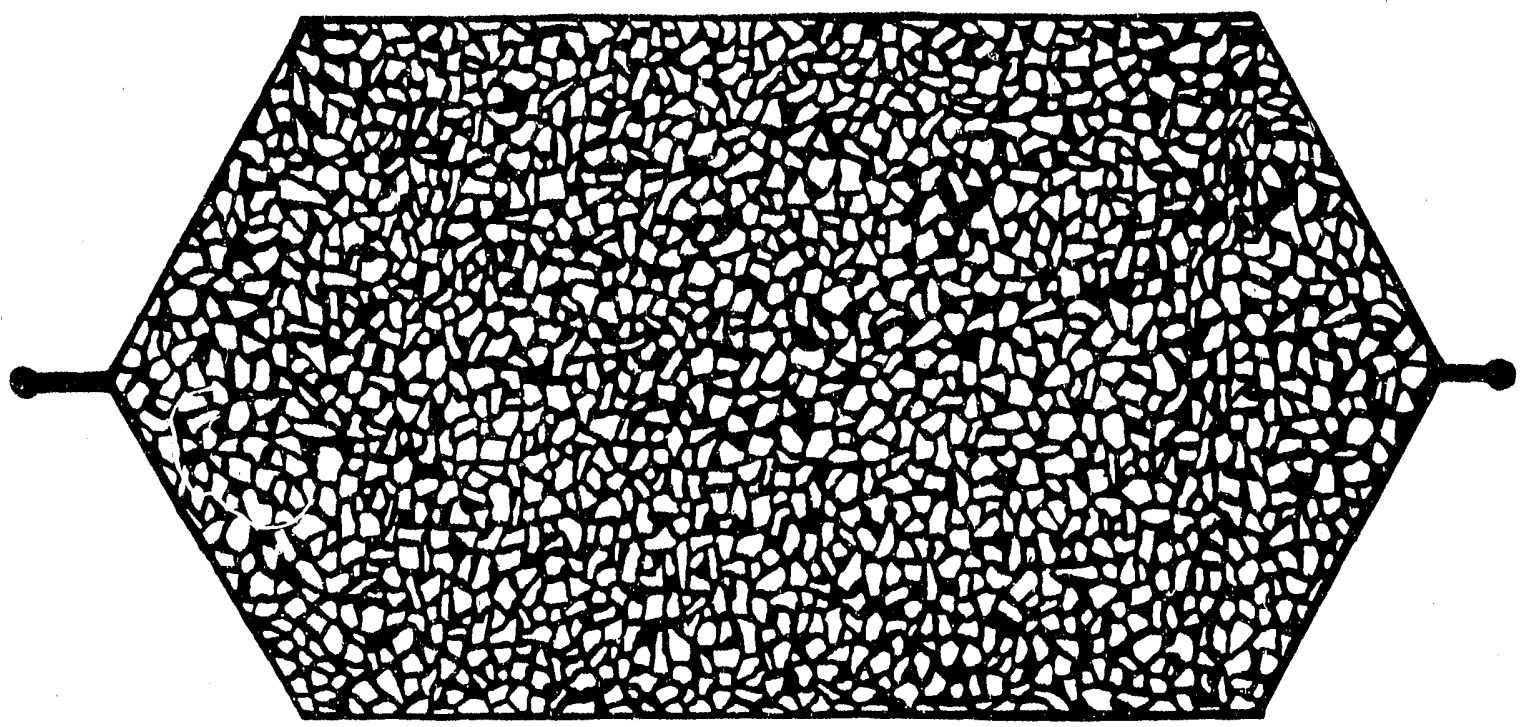

Fig. A-3. Homogeneous Rock Pattern

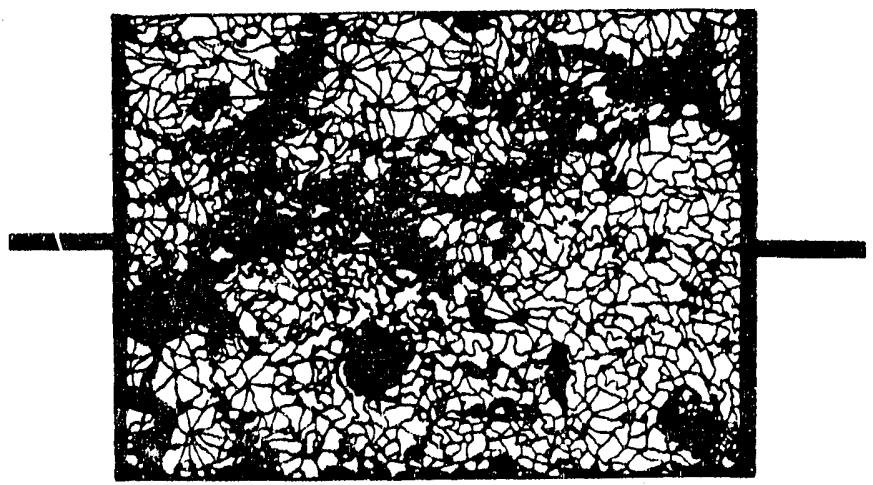

Fig. A-4. San Andres Carbonate Pattern 
If a pattern is to be etched into both the top and bottom plates, then two pieces of plate-glass mirror must be used per micromodel. The pattern will be etched into one plate, while the mirror image of the pattern will be etched into the complementary plate. If a pattern is to be etched into only one plate of the micromodel, then an unetched piece of plate glass is used as the complementary plate.

\section{Step 2. Removing the Mirror Backing}

There are two different solutions that can be used to remove the backing from the mirror. There is an epoxy stripper and an alkyd stripper. Try a sample piece of mirror in each solution to determine the best solution to use. The best solution will be the one that leaves a shiny clean copper surface upon which water will not bead.

\section{Step 2a. Alkyd Stripper}

This step should be performed in a fume hood. Use a glass or steel pan that is just large enough for the mirror to lie horizontally. Then cover the mirror with about one centimeter of stripper. Place the mirror in the solution with the backing side facing up. It should take 15 to 25 seconds for the backing to peel from the copper surface. When the backing is free of the copper, remove the mirror and rinse thoroughly with tap water. Once the stripping solution has been rinsed from the mirror, pour a liberal amount of paint remover onto the copper surface and rub with fingers until the copper is shiny. The copper is now ready to be rinsed.

\section{Step 2a. (alternate) Epoxy Stripoer}

This step should be performed in a fume hood. Apply a two-millimeter coat of the epoxy stripper on the mirror backing. Leave the stripper on the backing until the backing bubbles off the copper or for five minutes, whichever comes first. When the backing is free of the copper, rinse thoroughly with tap water. Now, pour a liberal amount of paint remover onto the copper surface and rub it into the copper with fingers until the copper is shiny. The copper is now ready to be rinsed.

\section{Step 2b. Rinse}

Rinse the paint remover from the mirror using tap water and apply a coat of a non-abrasive detergent (e.g., Sunlight( ). Leave the detergent on the copper for ten to twenty seconds. Rinse the copper with tap water and apply another coating of detergent. Rub the detergent into the copper gently with a sof $t$ wet sponge. Rinse the copper well with tap water. Immediately after rinsing, hoid the mirror vertically. If the water sheets, rinse with distilled water and blow the copper surface dry with compressed air. Then, prepare to coat with photo-resist. If the water beads on the copper, try washing the area with more detergent. If this does not work, apply some paint remover to the area. Then, rinse and wash with detergent; rinse with tap water; rinse with distilled water; and blow the copper surface dry with compressed air.

\section{Step 3. Applying the Photo-Resist}

Mix Kodak photo-resist concentrate (KTFR 146-1961) with rylene using a 1:2 ratio, respectively. Store the mixture in a dark bottle, and keep the bottle in a dark place when not in use. 
To apply the resist, hold the mirror horizontally with the copper side facing up, and pour on enough resist to coat the copper evenly. Leave the excess resist on the copper for ten seconds. Then, place the mirror vertically to drain most of the excess resist. Now, place the mirror vertically on a paper towel so that the bottom edge is on the towel and the top edge is leaining on something. Leave the mirror in a dark place for 20 to 30 minutes. After this time, place the mirror, resist-side up, in a dark place overnight. The mirror is now ready to be exposed.

\section{Step 4. Exposure}

Place the mirror under the printing lamp and put the pattern on the mirror with the emuision side on the resist. Then place a clear piece of glass on top of the pattern in order to hold the pattern in contact with the resist.

Most photo-resists need to be exposed with a UV lamp that has a peak wavelength of $365 \mathrm{~nm}$. The amount of energy that the lamp emits, as well as the distance the lamp is from the mirror, will control the time necessary for the correct exposure. The time needed for an eight-watt lamp six inches from the work is about five hours. A forty-watt lamp fifteen inches from the work needs about an hour, and a fifteen-hundred-watt lamp twenty-four inches from the work will need about ten seconds. However, the exact exposure time needs to be determined by trial and error. If the resist washes off the mirror during development, the resist was under exposed. If the resist stays on the mirror and the pattern does not show up during development, the resist was over exposed.

Exposure is the most critical part of the entire micromodel process. Collimated light is essential for a sharp image. The light can be collimated by using a collimated light source or by moving the lamp further away from the work. Unfortunately, by moving the lamp further away from the work, the intensity of the light on the work surface decreases, thus increasing the exposure time and $\mathrm{dec}^{\mathrm{a}}$ tasing feature resolution. Table $\mathrm{A} / \mathrm{gives}$ recommended exposure times for the patterns in Figs. A - 1 through A-4 when a 70-watt lamp (Spectroline Model XX-15A, Long Wave, UV-365 nm) is used to expose patterns at a distance of 11 inches from the light source.

TABLE A 1

Exposure Times for Patterns in Figs. A-1 through A-4

Pattern Exposure Time

Fig. 1 hour

Fig. 2 1 hour

Fig. $3 \quad 1$ hour

Fig. 4

Step 5. Development

After the mirror has been exposed, place it in an oven at $200^{\circ} \mathrm{F}$ for 30 minutes. Then remove the mirror and let it cool for 10 minutes. The remaining steps in this paragraph should be performed in a fume hood. Now, holding the mirror horizontally with the resist side up, pour enough xylene on the resist to cover the surface completely. Sligh/y tilt the mirror back and forth so that the xylene washes across the surface. Do not let the resist get dry! Pour on xylene as needed to keep the resist 
wet for 90 seconds. Once this time has elapsed, drain the excess xylene and then rinse the mirror under a gentle stream of tap water. Finally, gently rinse the resist with distilled water and place it back in the oven for 30 minutes.

Place the developed mirror in a $60 \%$ nitric acid solution for 15 seconds. Remove the mirror and rinse thoroughly with tap water, followed by a rinse with distilled water. Then, dry with compressed air. The pattern is either perfect, or the exposure time was incorrect. If the pattern is acceptable, all exposed glass that is not to be etched should be masked with resist or wax. This is done using a small paint brush. When the mask is fully dry, the mirror is ready to etch.

\section{Step 6. Etching}

The steps in this paragraph must be performed in a fume hood, and the worker must be fully protected with a mask and clothed with acid-resistant garments. The glass-etching solution used in this process will etch at a rate of $0.20 \mathrm{~mm} / \mathrm{hr}$. A good rule of thumb is that the depth of etch should be equal to the average throat width. However, the etch time should not exceed two hours as the mask will start to degrade. If a deeper etch is required, add proportionately more ammonium hydrogen difluoride and sulfuric acid to the solution. Of course, the trade-nff here is that the more concentrated the etch solution is, the rougher it will be on the photo-resist, copper, and mask. Now, place the mirror in a plastic container, add the etching solution, heat to $100^{\circ} \mathrm{F}$, and gently agitate-a stir bar works well for this.

NOTE: One liter of the glass etching solution will properly etch five $4^{n} \times 7^{n}$ mirrors. After this, the solution should be disposed.

When the desired depth of etch has been reached, remove the mirror and rinse it thoroughly with tap water. Then, scrub the photo-resist/metal vigorously using a wire brush or sandpaper. Ideally, the scrubbing will remove most of the brown or c pper material. Next, dip the mirror in $60 \%$ nitric acid for a few seconds to remove the silver. Then remove the remaining photo-resist and metal by placing the mirror in a very strong oxidizer such as concentrated sulfuric acid and Nochromix. [Nochromix is a trademark of Godax Laboratories. This is prepared using the vendor's procedure (1 size package/ 2 liters concentrated $\left.\mathrm{H}_{2} \mathrm{SO}_{4}\right)$.] The oxidizer solution should be heated $\left(\approx 60^{\circ} \mathrm{C}\right)$ and stirred while in contact with the mirror. Depending on how much photo-resist remains on the mirror, this cleaning process may take from 30 minutes to several hours. When all photo-resist and metal has been removed, rinse the model plate well.

\section{Step 7. Drilling Inlet and Outlet Holes}

Using a diamond bit, drill inlet and outlet holes (e.g., one-eighth-inch diameter) in the appropriate location in one of the etched plates. For each hole, we recommend drilling half way through a plate from the etched side of the plate. Then, finish drilling the hole from the unetched side of the plate. This procedure will maximize the chances of making an acceptable hole.

Step 8. Fusing

Make sure the model plate and the cover plate are clean and free of contamination. Align the two plates, and place a small drop of Supe. Glue at each corner to keep the plates from slipping. The fusing temperature is $690^{\circ} \mathrm{C}$ and the annealing temperature is $545^{\circ} \mathrm{C}$. A suggested temperature cycle for the fusing process is as follows: 


\begin{tabular}{cc} 
TABLE A2 \\
Suggested Temperature Cycle for Fusing \\
Temperature ( ${ }^{\circ}$ C) & Time (hrs) \\
\hline & \\
195 & 2.0 \\
370 & 2.0 \\
545 & 2.0 \\
690 & 2.0 \\
545 & 2.0 \\
370 & 2.0 \\
195 & 2.0 \\
off & 10.0
\end{tabular}

\section{CONCLUSION}

The purpose of this paper is to serve as a guideline for micromodel construction. Variations of this method may work equally well, and further improvements are certainly possible. Technique, attention to detail, patience, and creativity are the true keys to fabricating perfect micromodels.

\section{REFERENCES}

1. McKellar, M. and Wardlaw, N.C.: "A Method of Making Two-Dimensional Glass Micromodels of Pore Systems," tech. note, J. Canadian Pet. Tech. (1982) 21(4).

2. Chatzis, I.: "Photofabrication Technique of 2-D Glass Micromodels," PR.RC Report No. 8212, New Mexico Petroleum Recovery Research Center, Socorro, NM (Mar. 1982)。

3. Campbell, B.T: "Photofabrication of Porous Networks in Two-Dimensional Glass Plate Micromodels," PRRC Report No. 83-3, New Mexico Petroleum Recovery Research Center, Socorro, NM (1983).

4. Chatzis, I., Morrow, N.R., and Lim, H.T.: "Magnitude and Detailed Structure of Residual Oil Saturation," SPEJ (Apr. 1983) 311-326.

5. Bahralolom, I.M., Bretz, R.E., and Orr, F.M., Jr.: "Experirental Investigation of the Interaction of Phase Behavior With Microscopic Heterogeneity in a $\mathrm{CO}_{2}$ Flood," paper SPE 14147 presented at the 1985 SPE Technical Conference and Exhibition, Las Vegas, NV (Sept. 22-25).

6. Campbell, B.T. and Orr, F.M., Jr.: "Flow Visualization for $\mathrm{CO}_{2}$ /Crude-Oil Displacements," SPEJ (Oct. 1985) 665-678.

7. Bahralolom, 1.M. and Orr, F.M., Jr.: "Solubility and Extraction in Multiple-Contact Missible Displacements: Comparison of $\mathrm{N}_{2}$ and $\mathrm{CO}_{2}$ Flow Visualization Experiments," paper SPE 15079 presented at the 1986 SPE California Regional Meeting, Oakland, CA (Apr. 2-4). 
8. Martin, F.D. and Kovarik, F.S.: "Chemical Gels for Diverting $\mathrm{CO}_{2}$ : Baseline Experiments," paper SPE 16728 presented at the 1987 Technical Conference and Exhibition, Dallas, TX (Sept. 27-30).

9. Martin, F.D., Kovarik, F.S., Chang, P.W., and Phillips, J.C.: "Gels for $\mathrm{CO}_{2}$ Profile Modification," paper SPE/DOE 17330 presented at the 1988 SPE/DOE Symposium on Enhanced Oil Recovery, Tulsa, OK (Apr. 17-20).

10. Huh, D., Cochrane, T., and Kovarik, F.S.: "The Effect of Microscopic Heterogeneity on $\mathrm{CO}_{2}$ Foam Mobility--A Mechanistic Study," JPT (Aug. 1989) 872-879. 
APPENDIX B

GELATION STUDIES AT THE OPTIMUM REACTION pH 


\section{APPENDIX B \\ GELATION STUDIES AT THE OPTIMUM REACTION pH}

TABLE B 1

$6 \%$ Floperm 325 (3\% Floperm 325R, 3\% Floperm 325F), 0.5\% KCl

Gelation con titions: $105^{\circ} \mathrm{F}, \mathrm{pH}=9.0 \quad$ Gelation time: $10 \mathrm{hrs}$

Table Bla. Initial gel code/Gel code after 30 days

Initial pH

of Free Water

3

7

12.5

$\approx 70^{\circ} \mathrm{F}$
$\mathrm{I} / \mathrm{I}$
$\mathrm{I} / \mathrm{I}$
$\mathrm{I} / \mathrm{B}$

Temperature

$105^{\circ} \mathrm{F}$

I/I

$1 / 1$

$\mathrm{I} / \mathrm{H}$

$\frac{160^{\circ} \mathrm{F}}{\mathrm{I} / \mathrm{I}}$
$\mathrm{I} / \mathrm{I}$
$\mathrm{I} / \mathrm{G}$

$200^{\circ} \mathrm{F}$

$\mathrm{I} / \mathrm{I}$

I/I

$\mathrm{I} / \mathrm{H}$

Table B b. Gel volume after 30 days + Initial gel volume

\begin{tabular}{ccccc}
$\begin{array}{l}\text { Initial pH } \\
\text { of Free Water }\end{array}$ & $\frac{\mathbf{0} 0^{\circ} \mathrm{F}}{1.32}$ & $\begin{array}{c}\text { Temperature } \\
105^{\circ} \mathrm{F}\end{array}$ & $\frac{160^{\circ} \mathrm{F}}{1.39}$ & $\frac{200^{\circ} \mathrm{F}}{0.75}$ \\
\hline 3 & 1.26 & 1.39 & 1.39 & 0.84 \\
7 & 0.00 & 1.32 & 0.62 & 0.65
\end{tabular}

Table B1c, $\mathrm{pH}$ of free water after 30 days

\begin{tabular}{lcccr} 
Initial pH & \multicolumn{3}{c}{ Temperature } \\
of Free Water & $\approx 70^{\circ} \mathrm{F}$ & $\frac{105^{\circ} \mathrm{F}}{8.5}$ & $\frac{160^{\circ} \mathrm{F}}{7.2}$ & $\frac{200^{\circ} \mathrm{F}}{6.4}$ \\
\hline 3 & 7.6 & 8.0 & 7.2 & 6.4 \\
7 & 11.5 & 8.0 & 9.7 & 10.4
\end{tabular}


TABLE B2

$0.4 \%$ Xanthan (Flocon 4800), 100 ppm HCHO,

73 ppm $\mathrm{Cr}^{3+}(\mathrm{X}$-link 2000), $0.5 \% \mathrm{KCl}$

Gelation conditions: $105^{\circ} \mathrm{F}, \mathrm{pH}=4.2$

Table B2a, Initial gel code/Gel code after 30 days

\begin{tabular}{ll} 
Initial pH & \\
of Free Water & $\approx 70^{\circ} \mathrm{F}$ \\
\hline 3 & $\mathrm{I} / \mathrm{I}$ \\
7 & $\mathrm{I} / \mathrm{I}$ \\
12.5 & $\mathrm{I} / \mathrm{A}$
\end{tabular}

Gelation time: $8.5 \mathrm{hrs}$

Table B2b. Gel volume after 30 days + Initial gel volume

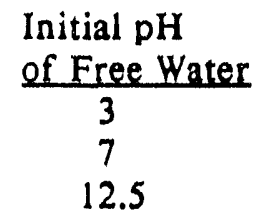

$\frac{\approx 0}{0} \mathrm{~F}$
1.08
1.08
0.00

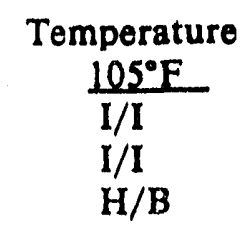

$\frac{160^{\circ} \mathrm{F}}{1 / \mathrm{I}}$

I/I

I/I

I/A $200^{\circ} \mathrm{F}$

H/G

I/H

I/A

Table $\mathrm{B} 2 \mathrm{c}, \mathrm{oH}$ of free water after 30 days

\begin{tabular}{|c|c|c|c|c|}
\hline Initial $\mathrm{pH}$ & & mperatu & & \\
\hline of Free Water & $\approx 70^{\circ} \mathrm{F}$ & $105^{\circ} \mathrm{F}$ & $160^{\circ} \mathrm{F}$ & $200^{\circ} \mathrm{F}$ \\
\hline 3 & 3.3 & 3.2 & 3.1 & 3.0 \\
\hline 7 & 3.5 & 3.3 & 3.0 & 3.2 \\
\hline 12.5 & 12.6 & 12.5 & 12.3 & 11.1 \\
\hline
\end{tabular}




\section{TABLE B3}

$0.4 \%$ Xanthan (Flocon 4800), 100 ppm HCHO, $154 \mathrm{ppm} \mathrm{Cr}^{3+}(\mathrm{X}$-link 2000), 0.5\% KCl

Gelation conditions: $105^{\circ} \mathrm{F}, \mathrm{pH}=3.9$

Table B3a. Initial gel code/Gel code after 30 days

Initial pH

of Free Water

3

7

12.5

$\approx 70^{\circ} \mathrm{E}$
$\mathrm{I} / \mathrm{I}$
$\mathrm{I} / \mathrm{I}$
$\mathrm{I} / \mathrm{G}$

I/G
Temperature

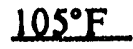

I/I

I/I

H/B

Gelation time: $6 \mathrm{hrs}$

\begin{tabular}{|c|c|}
\hline $160^{\circ} \mathrm{F}$ & $200^{\circ} \mathrm{F}$ \\
\hline $1 / I$ & $\mathrm{~J} / \mathrm{I}$ \\
\hline$I / I$ & $J / I$ \\
\hline $\mathrm{I} / \mathrm{A}$ & $\mathrm{J} / \mathrm{A}$ \\
\hline
\end{tabular}

Table B3b. Gel volumeafter 3'2 days + Initial gel volume

Initial $\mathrm{pH}$

of Free Water

3

7

12.5

$\frac{\approx 70^{\circ} \mathrm{F}}{0.72}$

0.92

\section{Temperature}

$\frac{105^{\circ} \mathrm{F}}{0.34}$

0.40

0.00 $\frac{100^{\circ} \mathrm{F}}{0.27}$

0.32

0.00 $200^{\circ} \mathrm{F}$

0.15

0.16

0.00

Table B3c $\mathrm{RH}$ of free water after 30 days

$\begin{array}{ccccc}\begin{array}{l}\text { Initial pH } \\ \text { of Free Water }\end{array} & \frac{\pi 70^{\circ} \mathrm{F}}{3.2} & \begin{array}{c}\text { Temperature } \\ 105^{\circ} \mathrm{F}\end{array} & \frac{160^{\circ} \mathrm{F}}{3.0} & \frac{200^{\circ} \mathrm{F}}{2.9} \\ 7 & 3.1 & 3.1 & 3.1 & 3.0 \\ 12.5 & 12.5 & 12.5 & 11.7 & 11.0\end{array}$


TABLE B4

$2.8 \%$ Polyacrylamide (Cyanagel 100 ),

500 ppm $\mathrm{Na}_{2} \mathrm{Cr}_{2} \mathrm{O}_{7}, 1500$ ppm Na $\mathrm{Na}_{2} \mathrm{O}_{4}, 0.5 \% \mathrm{KCl}$

Gelation conditions: $105^{\circ} \mathrm{F}, \mathrm{pH}=5.0$

Gelation time: $13.5 \mathrm{hrs}$

Table B4a. Initial gel code/Gel code after 30 days

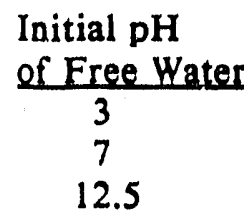

\begin{tabular}{lc}
$\approx 70^{\circ} \mathrm{F}$ & $\begin{array}{c}\text { Temperature } \\
105^{\circ} \mathrm{F}\end{array}$ \\
\hline $\mathrm{H} / \mathrm{H}$ & $\mathrm{H} / \mathrm{F}$ \\
$\mathrm{H} / \mathrm{H}$ & $\mathrm{H} / \mathrm{C}$ \\
$\mathrm{H} / \mathrm{B}$ & $\mathrm{H} / \mathrm{A}$
\end{tabular}

$160^{\circ} \mathrm{F}$

$200^{\circ} \mathrm{F}$

$\mathrm{H} / \mathrm{B}$

$\mathrm{H} / \mathrm{C} \quad \mathrm{H} / \mathrm{B}$

H/A H/A

Table B4b, Gel volume after 30 days + Initial gel volume

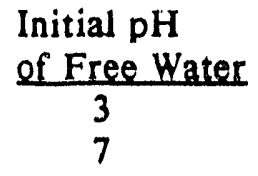

12.5

$\approx 70^{\circ} \mathrm{F}$
1.48
1.48
0.00

Temperature

$\frac{105^{\circ} \mathrm{F}}{1.29}$

1.54

0.00

1.72

1.72

0.00

$200^{\circ} \mathrm{F}$

1.32

1.32

0.00

Table $\mathrm{B} 4 \mathrm{c}, \mathrm{oH}$ of free water after 30 days

\begin{tabular}{|c|c|c|c|c|}
\hline Initial $\mathrm{pH}$ & & mperatu & & \\
\hline of Free Water & $\approx 70^{\circ} \mathrm{F}$ & $105^{\circ} \mathrm{F}$ & $160^{\circ} \mathrm{F}$ & $200^{\circ} \mathrm{F}$ \\
\hline 3 & 6.1 & 6.3 & 6.6 & 7.2 \\
\hline 7 & 6.4 & 0.4 & 6.6 & 7.3 \\
\hline 12.5 & 12.2 & 12.0 & 10.9 & 11.1 \\
\hline
\end{tabular}


TARLE B5

$0.994 \%$ Polyacrylamide (MARCIT), $150 \mathrm{ppm} \mathrm{Cr}^{3+}$ (as carboxylate from Marathon), $1 \% \mathrm{NaCl}$

Gelation conditions: $105^{\circ} \mathrm{F}, \mathrm{pH}=5.8$

Table 35a. Initial gel code/Gel code after 30 days

Initial pH
of Free Water
$\frac{3}{7}$
12.5

12.5

$\begin{array}{cc}\approx 70^{\circ} \mathrm{F} & \begin{array}{c}\text { Temperature } \\ \mathrm{H} / \mathrm{H}\end{array} \\ \mathrm{H} / \mathrm{H} & \mathrm{H} / \mathrm{H} \\ \mathrm{H} / \mathrm{C} & \mathrm{H} / \mathrm{H} \\ \mathrm{H} / \mathrm{B}\end{array}$

Table B5b. Gel volume after 30 days + Initial gel volume

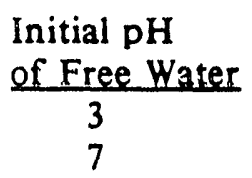

12.5

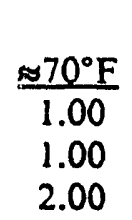

Temperature
$\frac{105^{\circ} \mathrm{F}}{0.89}$
1.00
2.00

Table B5c, $\mathrm{pH}$ of free water after 30 days

\author{
Temperature \\ $105^{\circ} \mathrm{F}$ \\ 5.8 \\ 12.0
}

Gelation time: $12.5 \mathrm{hrs}$

\begin{tabular}{ccccc}
$\begin{array}{l}\text { Initial pH } \\
\text { of Fret Water }\end{array}$ & $\frac{\pi 70^{\circ} \mathrm{F}}{5.1}$ & $\begin{array}{c}\text { Temperature } \\
105^{\circ} \mathrm{F}\end{array}$ & $\frac{160^{\circ} \mathrm{F}}{5.0}$ & $\frac{200^{\circ} \mathrm{F}}{6.2}$ \\
\hline 3 & 5.3 & 4.8 & 4.9 & 6.0 \\
12.5 & 12.2 & 5.0 & 10.9 & 10.8
\end{tabular}


TABLE B6

$1.39 \%$ Polyacrylamide (MARCIT),

$212 \mathrm{ppm} \mathrm{Cr}^{3+}$ (as carboxylate from Marathon), $1 \% \mathrm{NaCl}$

Gelation conditions: $105^{\circ} \mathrm{F}, \mathrm{pH}=5.8$

Gelation time: $11.5 \mathrm{hrs}$

Table B6a. Initial sel code/Gel code after 30 days

\begin{tabular}{ccccc}
$\begin{array}{l}\text { Initial pH } \\
\text { of Free Water }\end{array}$ & $\frac{\pi 70^{\circ} \mathrm{F}}{\mathrm{I}}$ & $\begin{array}{c}\text { Temperature } \\
105^{\circ} \mathrm{F}\end{array}$ & $\frac{160^{\circ} \mathrm{F}}{\mathrm{I}}$ & $\frac{200^{\circ} \mathrm{F}}{\mathrm{I}}$ \\
\hline 3 & $\mathrm{I} / \mathrm{I}$ & $\mathrm{I} / \mathrm{I}$ & $\mathrm{I}$ & $\mathrm{I} / \mathrm{I}$ \\
7 & $\mathrm{I} / \mathrm{I}$ & $\mathrm{I} / \mathrm{I}$ & $\mathrm{I}$ & $\mathrm{I} / \mathrm{B}$
\end{tabular}

Table B6b. Gel volume after 30 days + Initial gel volume

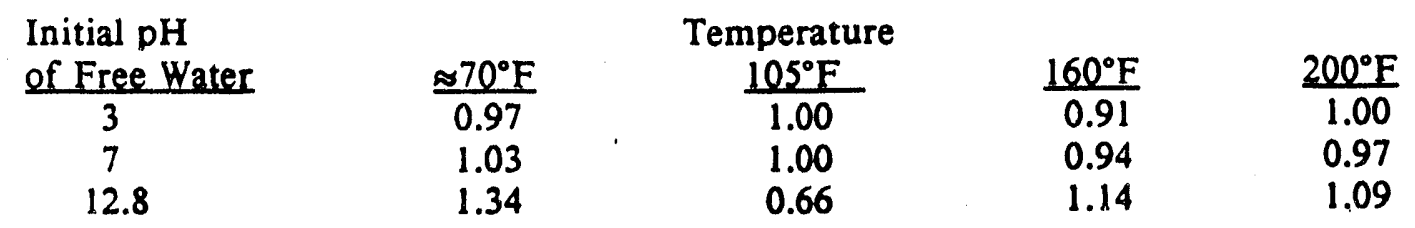

Table B6c. $\mathrm{pH}$ of free water after 30 days

\begin{tabular}{lccrr}
$\begin{array}{l}\text { Initial pH } \\
\text { of Free Water }\end{array}$ & $\approx 70^{\circ} \mathrm{F}$ & $\begin{array}{c}\text { Temperature } \\
105^{\circ} \mathrm{F}\end{array}$ & $\frac{160^{\circ} \mathrm{F}}{4.6}$ & $\frac{200^{\circ} \mathrm{F}}{5.8}$ \\
\hline 3 & 5.0 & 4.8 & 4.7 & 5.9 \\
7 & 5.0 & 5.5 & 10.5 & 10.1
\end{tabular}


2.5\% Floperm 465P, 3\% Floperm $465 \mathrm{~B}_{2}$,

$0.25 \%$ Floperm $465 \mathrm{X}, 0.5 \% \mathrm{KCl}$

Gelation conditions: $105^{\circ} \mathrm{F}, \mathrm{pH}=5.1$

Table B7a. Initial gel code/Gel code after 30 days

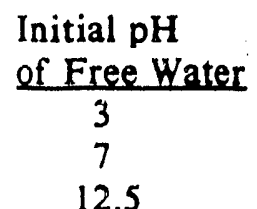

12.5

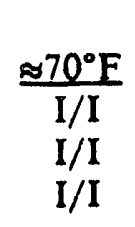

Temperature

$105^{\circ} \mathrm{F}$

I/I

I/H

I/I
Gelation time: $14.5 \mathrm{hrs}$

Table B7E. Gel volume after 30 days + Initial gel volume

\begin{tabular}{lcccc}
$\begin{array}{l}\text { Initial pH } \\
\text { of Free Water }\end{array}$ & $\frac{\approx 70^{\circ} \mathrm{F}}{0.49}$ & $\frac{105^{\circ} \mathrm{F}}{0.15}$ & $\frac{160^{\circ} \mathrm{F}}{0.14}$ & $\frac{200^{\circ} \mathrm{F}}{0.10}$ \\
\hline 3 & 0.55 & 0.14 & 0.10 & 0.10 \\
12.5 & 0.68 & 0.24 & 0.14 & 0.10
\end{tabular}

Table $\mathrm{B} 7 \mathrm{c}$. $\mathrm{pH}$ of free water after 30 days

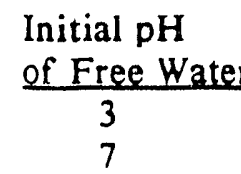

12.5

$\approx 70^{\circ} \mathrm{F}$
4.0
4.1
5.0

5.0

Temperature
$\frac{105^{\circ} \mathrm{F}}{4.0}$
4.0
5.0

$\underline{160^{\circ} \mathrm{F}}$

4.6

4.2

5.0 $200^{\circ} \mathrm{F}$

I/I

I/I

$\begin{array}{cc}\frac{160^{\circ} \mathrm{F}}{\mathrm{I} / \mathrm{I}} & \frac{200^{\circ} \mathrm{F}}{\mathrm{I} / \mathrm{I}} \\ \mathrm{I} / \mathrm{I} & \mathrm{I} / \mathrm{I} \\ \mathrm{I} / \mathrm{I} & \mathrm{I} / \mathrm{I}\end{array}$

0.10

0.10 
TABLE B8

$10 \%$ Colloidal Silica (Ludox HS-40), 2.1\% NaCl

Gelation conditions: $105^{\circ} \mathrm{F}, \mathrm{pH}=8.25$

Gelation time: $9.5 \mathrm{hrs}$

Table B8a. Initial gel code/Gel code after 30 days
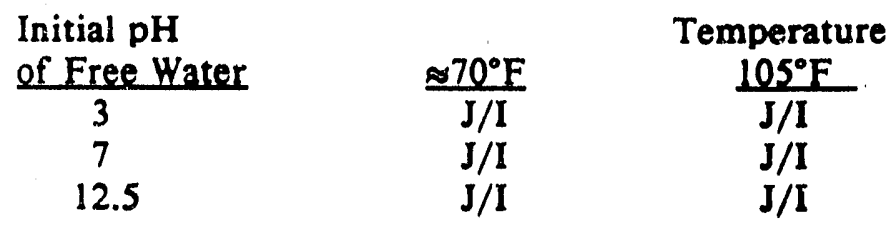

$160^{\circ} \mathrm{F}$

$\mathrm{J} / \mathrm{I}$

$\mathrm{J} / \mathrm{I}$

$\mathbf{J} / \mathbf{I}$

$200^{\circ} \mathrm{F}$

$\mathrm{J} / \mathrm{I}$

$\mathrm{J} / \mathbf{I}$

$\mathbf{J} / \mathbf{I}$

Table B8b. Gel volume after 30 days + Initial gel volume

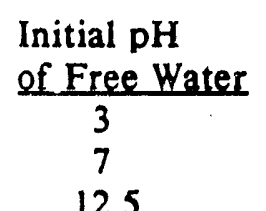

12.5

$\approx 70^{\circ} \mathrm{F}$
1.17
1.17
0.92

1.17
0.92
Temperature

$\frac{105^{\circ} \mathrm{F}}{1.14}$
1.20
0.92

$\begin{array}{cc}\frac{160^{\circ} \mathrm{F}}{1.17} & \frac{200^{\circ} \mathrm{F}}{1.26} \\ 1.14 & 1.11 \\ 0.92 & 0.92\end{array}$

Table $\mathrm{B8c}, \mathrm{pH}$ of free water after 30 days

\begin{tabular}{lcccc}
$\begin{array}{l}\text { Initial pH } \\
\text { of Free Water }\end{array}$ & \multicolumn{5}{c}{$\begin{array}{c}\text { Temperature } \\
\text { (70 } \mathrm{F}\end{array}$} & $\frac{105^{\circ} \mathrm{F}}{8.4}$ & $\frac{160^{\circ} \mathrm{F}}{8.5}$ & $\frac{200^{\circ} \mathrm{F}}{6.2}$ \\
\hline 3 & 7.6 & 8.4 & 8.6 & 6.3 \\
12.5 & 8.0 & 10.8 & 10.8 & 10.8
\end{tabular}


TABLE B9

$10 \%$ Colloidal Silica (Ludox SM), $0.7 \% \mathrm{NaCl}$

Gelation conditions: $105^{\circ} \mathrm{F}, \mathrm{pH}=8.25$

Gelation time: $31 \mathrm{hrs}$

Table B9a. Initial gel code/Gel code after 30 davs

\begin{tabular}{|c|c|c|c|c|}
\hline & & Temperature & & \\
\hline$\frac{\text { of Free Water }}{3}$ & $\frac{\approx 70^{\circ} \mathrm{F}}{\mathrm{J} / \mathrm{I}}$ & $\frac{105^{\circ} \mathrm{F}}{J I}$ & $\frac{100 \mathrm{~F}}{\mathrm{~J} / \mathrm{I}}$ & $\frac{20 \mathrm{~V} / \mathrm{J}}{\mathrm{J} / \mathrm{I}}$ \\
\hline $\begin{array}{l}3 \\
7\end{array}$ & $\mathrm{~J} / \mathrm{J}$ & $\begin{array}{l}\mathrm{J} / \mathrm{J} \\
\mathrm{J} / \mathrm{J}\end{array}$ & J/I & J/I \\
\hline 12.5 & $\mathrm{~J} / \mathrm{I}$ & J/I & $\mathrm{J} / \mathbf{I}$ & $\mathrm{J} / \mathrm{I}$ \\
\hline
\end{tabular}

Table B9b. Gel volume after 30 days + Initial gel volume

\begin{tabular}{ccccc} 
Initial pH & \multicolumn{4}{c}{ Temperature } \\
of Free Water & $\frac{\approx 70^{\circ} \mathrm{F}}{0.92}$ & $\frac{105^{\circ} \mathrm{F}}{0.93}$ & $\frac{160^{\circ} \mathrm{F}}{0.92}$ & $\frac{200^{\circ} \mathrm{F}}{0.97}$ \\
\hline 3 & 0.94 & 0.92 & 0.92 & 0.95 \\
7 & 0.78 & 0.76 & 0.79 & 0.84
\end{tabular}

Table $\mathrm{B} 9 \mathrm{c}, \mathrm{pH}$ of free water after 30 days

\begin{tabular}{lccrr}
$\begin{array}{l}\text { Initial pH } \\
\text { Of Free Water }\end{array}$ & $\approx 70^{\circ} \mathrm{F}$ & $\begin{array}{c}\text { Temperature } \\
\frac{105^{\circ} \mathrm{F}}{8.0}\end{array}$ & $\frac{160^{\circ} \mathrm{F}}{9.1}$ & $\frac{200^{\circ} \mathrm{F}}{9.4}$ \\
\hline 3 & 8.0 & 8.6 & 9.1 & 9.4 \\
12.5 & 8.2 & 8.7 & 10.9 & 11.0
\end{tabular}


APPENDIX C

GELATION STUDIES AT $\mathrm{pH}=7$ 


\section{APPENDIX C \\ GELATION STUDIES AT $\mathrm{pH}=7$}

TABLE Cl

$6 \%$ Floperm 325 (3\% Floperm 325R, 3\% Floperm 325F), $0.5 \% \mathrm{KCl}$

Gelation conditions: $105^{\circ} \mathrm{F}, \mathrm{pH}=7$

Table Cla. Initial gel code/Gel code after 30 days

Initial pH

$\frac{\text { of Free Water }}{7}$

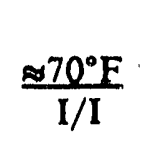

$\mathrm{I} / \mathrm{I}$
Temperature

$\frac{105^{\circ} \mathrm{F}}{\mathrm{I} / \mathrm{I}}$ $160^{\circ} \mathrm{E}$

I/I $\underline{200^{\circ} \mathrm{F}}$

I/I

Table Clb. Gel volume after 30 days + Initial gel volume

\begin{tabular}{llcrl} 
Initial $\mathrm{pH}$ & \multicolumn{3}{c}{ Temperature } \\
$\frac{\text { of Free Water }}{7}$ & $\frac{\approx 70^{\circ} \mathrm{F}}{1.14}$ & $\frac{105^{\circ} \mathrm{F}}{1.17}$ & $\frac{160^{\circ} \mathrm{F}}{1.14}$ & $\frac{200^{\circ} \mathrm{F}}{1.17}$
\end{tabular}

Table $\mathrm{Clc}$. $\mathrm{pH}$ of free water after 30 davs

Initial $\mathrm{pH}$

of Free Water

7 $\frac{\approx 70^{\circ} \mathrm{F}}{4.5}$
Temperature

$$
\frac{105^{\circ} \mathrm{F}}{4.0}
$$

$\frac{160^{\circ} \mathrm{F}}{3.3}$

3.3 $\underline{200^{\circ} \mathrm{F}}$

3.25 
TABLE C2

$0.4 \%$ Xanthan (Flocon 4800 ), 100 ppm HCHO, $73 \mathrm{ppm} \mathrm{Cr}^{3+}(\mathrm{X}$-link 2000), 0.5\% KCl

Gelation conditions: $105^{\circ} \mathrm{F}, \mathrm{pH}=7$

Gelation time: 7.25 hrs

Table C2a. Initial gel code/Gel code after 30 days

Initial pH

of Free Water

7

$\frac{\approx 70^{\circ} \mathrm{F}}{\mathrm{E} / \mathrm{C}}$

Temperature

$\frac{105^{\circ} \mathrm{F}}{\mathrm{E} / \mathrm{B}}$

$160^{\circ} \mathrm{F}$

$\mathrm{E} / \mathrm{B}$

$\frac{200^{\circ} \mathrm{F}}{\mathrm{E} / \mathrm{B}}$

Table C2b. Gel volume after 30 days + Initial gel volume

Initial $\mathrm{pH}$

of Free Water

7 $\frac{\$ 70^{\circ} \mathrm{F}}{1.14}$
Temperature

$105^{\circ} \mathrm{F}$

1.17

$160^{\circ} \mathrm{F}$

$200^{\circ} \mathrm{F}$

0.15

Table $\mathrm{C} 2 \mathrm{c}, \mathrm{pH}$ of free water after 30 days

Initial pH

of Free Water

7 $\frac{\approx 70^{\circ} \mathrm{F}}{5.8}$

\section{Temperature}

$105^{\circ} \mathrm{F}$

6.4 $\frac{160^{\circ} \mathrm{F}}{5.5}$

$\frac{200^{\circ} \mathrm{F}}{5.2}$ 
TABLE C3

$0.4 \%$ Xanthan (Flocon 4800), 100 ppm HCHO, $154 \mathrm{ppm} \mathrm{Cr}^{3+}(\mathrm{X}$-link 2000), 0.5\% KCl

Gelation conditions: $105^{\circ} \mathrm{F}, \mathrm{pH}=7$

Table C3a. Initial sel code/Gel code after 30 days

Initial pH

of Free Water

7

$\frac{70^{\circ} \mathrm{F}}{\mathrm{I} / \mathrm{I}}$

Temperature

I/I

Table C3b, Gel volume after 30 days + Initial gel volume

Initial pH

of Free Water

7
$\approx 70^{\circ} \mathrm{F}$
0.95

Temperature

$$
\frac{105^{\circ} \mathrm{F}}{0.79}
$$

$160^{\circ} \mathrm{F}$

0.84

$\frac{200^{\circ} \mathrm{F}}{0.62}$

0.62

Table C3c, oH of free water after 30 days

Initial $\mathrm{pH}$

of Free Water

$\frac{\$ 70^{\circ} \mathrm{F}}{4.8}$
Tempẹature
5.5

$105^{\circ} \mathrm{F} \quad 160^{\circ} \mathrm{F}$

5.0

$\frac{200^{\circ} \mathrm{F}}{4.6}$ 
TABLE C4

$2.8 \%$ Polyacrylamide (Cyanagel 100),

500 ppm $\mathrm{Na}_{2} \mathrm{Cr}_{2} \mathrm{O}_{7}, 1500$ ppm Na $\mathrm{S}_{2} \mathrm{O}_{4}, 0.5 \% \mathrm{KCl}$

Gelation conditions: $105^{\circ} \mathrm{F}, \mathrm{pH}=7$

Gelation time: no gelation seen

Table C4a. Initial gel code/Gel code after 30 days

Initial $\mathrm{pH}$

$\frac{\text { of Free Water }}{7}$

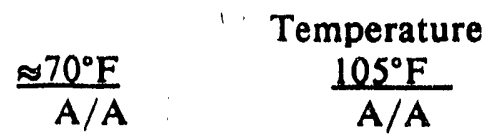

$\frac{160^{\circ} \mathrm{F}}{\mathrm{A} / \mathrm{A}}$

$\frac{200^{\circ} \mathrm{F}}{\mathrm{A} / \mathrm{A}}$

Table C4b Gel volume after 30 days + Initial gel volume

Initial $\mathrm{pH}$

of Free Water

7

$\approx 70^{\circ} \mathrm{F}$

Temperature

$105^{\circ} \mathrm{F}$

$\underline{160^{\circ} \mathrm{F}}$

$\underline{200^{\circ} \mathrm{F}}$

-.. -..

Table $\mathrm{C} 4 \mathrm{c}, \mathrm{pH}$ of free water after 30 days

Initial $\mathrm{pH}$

of Free Water

7 $\frac{\approx 70^{\circ} \mathrm{F}}{7.0}$
Temperature
$\frac{105^{\circ} \mathrm{F}}{7.3}$

$\frac{160 \%}{8.0}$

$\frac{200}{8.4}$ 
TABLE C5

0.994\% Polyacrylamide (MARCIT),

$150 \mathrm{ppm} \mathrm{Cr}^{3+}$ (as carboxylate from Marathon), $1 \% \mathrm{NaCl}$

Gelation conditions: $105^{\circ} \mathrm{F}, \mathrm{pH}=7$

Table C5a. Initial sel code/Gel code after 30 days

Initial $\mathrm{pH}$

of Free Water

7

$$
\frac{\approx 70^{\circ} \mathrm{F}}{\mathrm{H} / \mathrm{H}}
$$

\section{Temperature}

$\frac{105^{\circ} \mathrm{F}}{\mathrm{H} / \mathrm{H}}$
Gelation time: $11 \mathrm{hrs}$

Table C5b. Gel volume after 30 days + Initial gel volume

Initial $\mathrm{pH}$

of Free Water

7 $\frac{\approx 70^{\circ} \mathrm{F}}{0.97}$
Temperature

$\frac{105^{\circ} \mathrm{F}}{0.90}$

$160^{\circ} \mathrm{F}$

$200^{\circ} \mathrm{F}$

$\mathrm{H} / \mathrm{H}$

$\mathrm{H} / \mathrm{H}$

Table C5c, oH of free water after 30 days

Initial $\mathrm{pH}$

of Free Water

7
Temperature

$\frac{105^{\circ} \mathrm{F}}{5.1}$ $\frac{160^{\circ} \mathrm{F}}{0.92}$

0.92 $200^{\circ} \mathrm{F}$

0.97 
TABLE C6

1.39\% Polyacrylamide (MARCIT),

$212 \mathrm{ppm} \mathrm{Cr}^{3+}$ (as carboxylate from Marathon), $1 \% \mathrm{NaCl}$

Gelation conditions: $105^{\circ} \mathrm{F}, \mathrm{pH}=7$

Gelation time: 9.5 hrs

Table C6a. Initial gel code/Gel code after 30 days

Initial $\mathrm{pH}$

Temperature

of Free Water

$\begin{array}{cc}\simeq 70^{\circ} \mathrm{F} & \begin{array}{c}\text { Temperature } \\ \mathrm{I} / \mathrm{I}\end{array} \\ \frac{105^{\circ} \mathrm{F}}{\mathrm{I} / \mathrm{I}}\end{array}$

$\frac{160^{\circ} \mathrm{F}}{\mathrm{I} / \mathrm{I}}$

$\frac{200^{\circ} \mathrm{F}}{\mathrm{I} / \mathrm{I}}$

Table C6b. Gel volume after 30 davs + Initial sel volume
Initial $\mathrm{pH}$
of Free Water
7
$\frac{\approx 70^{\circ} \mathrm{F}}{1.00}$
Temperature
$\frac{105^{\circ} \mathrm{F}}{1.00}$
$160^{\circ} \mathrm{F}$
$\frac{200^{\circ} \mathrm{F}}{1.06}$
1.06

Table $\mathrm{C6e}, \mathrm{pH}$ of free water after 30 days

Initial $\mathrm{pH}$

of Free Water $\frac{\approx 70^{\circ} \mathrm{F}}{5.3}$
Temperature
$\frac{105^{\circ} \mathrm{F}}{5.0}$

$\frac{160^{\circ} \mathrm{F}}{4.9}$

4.9

$\frac{200^{\circ} \mathrm{F}}{6.0}$

6.0 
TABLE C7

2.5\% Floperm 465P, 3\% Floperm $465 \mathrm{~B}_{2}$,

$0.25 \%$ Floperm $465 \mathrm{X}, 0.5 \% \mathrm{KCl}$

Gelation conditions: $105^{\circ} \mathrm{F}, \mathrm{pH}=7$

Table C7a. Initial gel code/Gel code after 30 days

Initial $\mathrm{pH}$

of Free Water 7 $\frac{\approx 70^{\circ} \mathrm{F}}{\mathrm{A} / \mathrm{A}}$
Temperature

$\frac{105^{\circ} \mathrm{F}}{\mathrm{A} / \mathrm{A}}$
Gelation time: no gelation seen

Table C7b. Gel volume after 30 days + Initial gel volume

Initial $\mathrm{pH}$

of Free Water

$\approx 70^{\circ} \mathrm{F}$

Temperature

$105^{\circ} \mathrm{F}$

$160^{\circ} \mathrm{F}$

$200^{\circ} \mathrm{F}$

$\frac{160^{\circ} \mathrm{F}}{\mathrm{A} / \mathrm{A}}$

$\frac{200^{\circ} \mathrm{F}}{\mathrm{A} / \mathrm{A}}$

7

free water after 30 days

$\frac{\text { of Free Water }}{7}$

$\frac{\approx 70^{\circ} \mathrm{F}}{7.2}$

Temperature
$\frac{105^{\circ} \mathrm{F}}{6.9}$

$\frac{160^{\circ} \mathrm{F}}{6.5}$

$\frac{200^{\circ} \mathrm{F}}{6.4}$ 
TABLE C8

$10 \%$ Colloidal Silica (Ludox HS-40), $2.1 \% \mathrm{NaCl}$

Gelation conditions: $105^{\circ} \mathrm{F}, \mathrm{pH}=7$

Table C8a. Initial gel code/Gel code after 30 days

Initial $\mathrm{pH}$

of Free Water

7

$\frac{\approx 70^{\circ} \mathrm{F}}{\mathrm{J} / \mathrm{I}}$
Temperature

$\frac{105^{\circ} \mathrm{F}}{\mathrm{J} / \mathrm{I}}$

Gelation time: 6.5 h:s

$\frac{160^{\circ} \mathrm{F}}{\mathrm{J} / \mathrm{I}} \quad \frac{200^{\circ} \mathrm{F}}{\mathrm{J} / \mathrm{I}}$

Table C8b Gel volume after 30 davs + Initial gel volume

\begin{tabular}{llcrr} 
Initial pH & \multicolumn{3}{c}{ Temperature } \\
of Free Water & $\frac{\approx 70^{\circ} \mathrm{F}}{1.20}$ & $\frac{105^{\circ} \mathrm{F}}{1.17}$ & $\frac{160^{\circ} \mathrm{F}}{1.17}$ & $\frac{200^{\circ} \mathrm{F}}{1.23}$
\end{tabular}

Table $\mathrm{C} 8 \mathrm{c}$. $\mathrm{pH}$ of free water after 30 days

\begin{tabular}{llcll} 
Initial pH & \multicolumn{3}{c}{ Temperature } \\
of Free Water & $\frac{\approx 70^{\circ} \mathrm{F}}{7.0}$ & $\frac{105^{\circ} \mathrm{F}}{7.5}$ & $\frac{160^{\circ} \mathrm{F}}{7.8}$ & $\frac{200^{\circ} \mathrm{F}}{7.9}$
\end{tabular}


$10 \%$ Colloidal Silica (Ludox SM), $0.7 \% \mathrm{NaCl}$

Gelation conditions: $105^{\circ} \mathrm{F}, \mathrm{pH}=7$

Table C2a. Initial gel code/Gel code af ter 30 days

Initial $\mathrm{pH}$

of Free Water

7

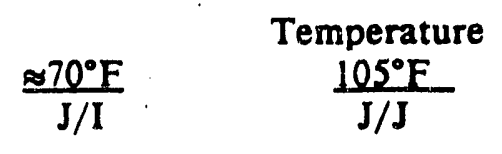

Table C9b Gel volume after 30 days + Initial gel volume

Initial $\mathrm{pH}$

of Free Water
Gelation time: $4.5 \mathrm{hrs}$

Table C9e, phi of free water after 30 davs

Initial $\mathrm{pH}$

of Free Water $\frac{\approx 70^{\circ} \mathrm{F}}{7.2}$
Temperature

$$
\frac{105^{\circ} \mathrm{F}}{0.95}
$$

$\frac{160^{\circ} \mathrm{F}}{0.92}$

0.92

$\frac{200^{\circ} \mathrm{F}}{0.95}$

0.95 $\frac{200^{\circ} \mathrm{F}}{\mathrm{J} / \mathrm{I}}$
Temperature $105^{\circ} \mathrm{F}$
7.8

$\frac{160^{\circ} \mathrm{F}}{8.7}$

$200^{\circ} \mathrm{F}$

9.1 


\section{APPENDIX D}

RESULTS FROM CORE EXPERIMENTS WITH

RESORCINOL-FORMALDEHYDE GELANTS 


\section{APPENDIX D \\ RESULTS FROM CORE EXPERIMENTS WITH RESORCINOL-FORMALDEHYDE GELANTS}

TABLE DI

Results From Brine Injection After Gelation of $3 \%$ Resorcinol, $3 \%$ Formaldehyde, $105^{\circ} \mathrm{F}$

\begin{tabular}{|c|c|c|c|c|c|}
\hline Rock & Brine & $\begin{array}{c}\text { Flux } \\
\text { (ft/d) }\end{array}$ & $\begin{array}{l}\text { Pressure } \\
\text { Gradient } \\
\text { (osi/ft) }\end{array}$ & $\begin{array}{l}\text { Final } \\
k \\
\text { (md) }\end{array}$ & $\underline{F_{\pi}}$ \\
\hline $\begin{array}{l}390-\mathrm{md} \\
\text { Berea } \\
\text { Sandstone } \\
\phi=0.188\end{array}$ & $\begin{array}{l}0.5 \% \mathrm{KCl} \\
\mathrm{pH}=7 \\
\mu_{w}=0.65 \mathrm{cp}\end{array}$ & $\begin{array}{l}15.47 \\
3.094 \\
0.619\end{array}$ & $\begin{array}{r}298 \\
63 \\
14\end{array}$ & $\begin{array}{l}5.3 \\
5.0 \\
4.6\end{array}$ & $\begin{array}{l}73.6 \\
77.6 \\
85.7\end{array}$ \\
\hline $\begin{array}{l}57-\mathrm{md} \\
\text { Berea } \\
\text { Sandstone } \\
\phi=0.182\end{array}$ & $\begin{array}{l}0.5 \% \mathrm{KCl} \\
\mathrm{pH}=7 \\
\mu_{\mathrm{w}}=0.65 \mathrm{cp}\end{array}$ & $\begin{array}{l}0.622 \\
3.112 \\
9.337 \\
3.112 \\
0.622 \\
0.124 \\
0.025\end{array}$ & $\begin{array}{r}160 \\
771 \\
2130 \\
727 \\
142 \\
29 \\
6\end{array}$ & $\begin{array}{l}0.40 \\
0.42 \\
0.45 \\
0.44 \\
0.45 \\
0.44 \\
0.45\end{array}$ & $\begin{array}{l}143 \\
138 \\
127 \\
130 \\
127 \\
131 \\
127\end{array}$ \\
\hline $\begin{array}{l}13-\text { md } \\
\text { Indiana } \\
\text { Limestone } \\
\phi=0.179\end{array}$ & $\begin{array}{l}0.5 \% \mathrm{KCl} \\
\mathrm{pH}=7 \\
\mu_{w}=0.65 \mathrm{cp}\end{array}$ & $\begin{array}{c}0.622 \\
3.109 \\
0.622 \\
3.109 \\
15.55\end{array}$ & $\begin{array}{r}24 \\
115 \\
24 \\
111 \\
504\end{array}$ & $\begin{array}{l}2.7 \\
2.8 \\
2.7 \\
2.9 \\
3.0\end{array}$ & $\begin{array}{l}4.8 \\
4.7 \\
4.8 \\
4.5 \\
4.1\end{array}$ \\
\hline $\begin{array}{l}570-\mathrm{md} \\
\text { Berea } \\
\text { Sandstone } \\
\phi=0.223\end{array}$ & $\begin{array}{l}0.5 \% \mathrm{KCl} \\
0.05 \mathrm{M} \mathrm{NaHCO} \\
\mathrm{pH}=9 \\
\mu_{w}=0.67 \mathrm{cp}\end{array}$ & $\begin{array}{l}0.621 \\
3.103 \\
0.621 \\
0.124 \\
0.621 \\
3.103\end{array}$ & $\begin{array}{r}251 \\
750 \\
154 \\
29 \\
140 \\
664\end{array}$ & $\begin{array}{l}0.26 \\
0.44 \\
0.43 \\
0.45 \\
0.47 \\
0.50\end{array}$ & $\begin{array}{l}2170 \\
1300 \\
1330 \\
1270 \\
1216 \\
1151\end{array}$ \\
\hline $\begin{array}{l}7.4-\mathrm{md} \\
\text { Indiana } \\
\text { Limestone } \\
\phi=0.174\end{array}$ & $\begin{array}{l}0.5 \% \mathrm{KCl} \\
0.05 \mathrm{M} \mathrm{NaHCO} \\
\mathrm{pH}=9 \\
\mu_{w}=0.67 \mathrm{cp}\end{array}$ & $\begin{array}{l}0.025 \\
0.012 \\
0.006\end{array}$ & $\begin{array}{r}1006 \\
569 \\
302\end{array}$ & $\begin{array}{l}0.0026 \\
0.0022 \\
0.0021\end{array}$ & $\begin{array}{l}2800 \\
3300 \\
3500\end{array}$ \\
\hline
\end{tabular}


TABLE D2

Results During Brine Injection After Gel Placement in 455-md Berea Sandstone (Gelant contains $3 \%$ resorcinol, $3 \%$ formaldehyde, $0.5 \% \mathrm{KCl}, 0.05 \mathrm{M}$ phosphate, $\mathrm{pH}=7$ ) $\phi=0.224, \mu_{w}=0.70 \mathrm{cp}, 105^{\circ} \mathrm{F}$

\begin{tabular}{|c|c|c|c|c|}
\hline $\begin{array}{c}\text { Flux } \\
\text { (ft/d) }\end{array}$ & $\begin{array}{l}\text { Pressure } \\
\text { Gradient } \\
\text { (psi/ft) }\end{array}$ & $\begin{array}{l}\text { Final } \\
k \\
\text { (md) }\end{array}$ & $\mathrm{F}_{\mathrm{r}}$ & \\
\hline 0.025 & 18.2 & 0.152 & 3000 & \\
\hline $\begin{array}{l}0.050 \\
0.025\end{array}$ & $\begin{array}{l}28.0 \\
17.6\end{array}$ & $\begin{array}{l}0.198 \\
0.157\end{array}$ & $\begin{array}{l}2300 \\
2900\end{array}$ & $F_{r}=845 u^{-0.33}$ \\
\hline $\begin{array}{l}0.201 \\
0.025 \\
0.100\end{array}$ & $\begin{array}{r}56.2 \\
8.8 \\
30.6\end{array}$ & $\begin{array}{l}0.396 \\
0.314 \\
0.361\end{array}$ & $\begin{array}{l}1150 \\
1450 \\
1260\end{array}$ & $F_{r r}=970 u^{-0.11}$ \\
\hline $\begin{array}{l}0.393 \\
0.025 \\
0.100\end{array}$ & $\begin{array}{r}73.6 \\
7.9 \\
26.3\end{array}$ & $\begin{array}{l}0.591 \\
0.350 \\
0.421\end{array}$ & $\begin{array}{r}770 \\
1300 \\
1080\end{array}$ & $F_{r r}=662 u^{-0.19}$ \\
\hline $\begin{array}{l}0.785 \\
0.100 \\
0.025\end{array}$ & $\begin{array}{r}100 \\
18.0 \\
5.8\end{array}$ & $\begin{array}{l}0.868 \\
0.615 \\
0.474\end{array}$ & $\begin{array}{l}524 \\
740 \\
960\end{array}$ & $F_{n}=500 u^{-0.17}$ \\
\hline $\begin{array}{l}1.570 \\
0.393 \\
0.025\end{array}$ & $\begin{array}{r}147 \\
38.0 \\
3.5\end{array}$ & $\begin{array}{l}1.182 \\
1.143 \\
0.789\end{array}$ & $\begin{array}{l}385 \\
398 \\
577\end{array}$ & $F_{r r}=386 u^{-0.10}$ \\
\hline $\begin{array}{c}15.70 \\
6.280 \\
3.140 \\
1.570 \\
0.785 \\
0.393 \\
0.100 \\
0.050 \\
0.025\end{array}$ & $\begin{array}{r}1103 \\
454 \\
218 \\
76.0 \\
53.3 \\
27.9 \\
6.6 \\
3.3 \\
1.8\end{array}$ & $\begin{array}{l}1.574 \\
1.532 \\
1.591 \\
1.522 \\
1.631 \\
1.558 \\
1.672 \\
1.691 \\
1.564\end{array}$ & $\begin{array}{l}289 \\
297 \\
286 \\
299 \\
279 \\
292 \\
272 \\
269 \\
291\end{array}$ & $\bar{F}_{\pi}=286$ \\
\hline
\end{tabular}


TABLE D3

Results From Brine Injection After Gel Placement in 7-md Indiana Limestone (Gelant contains $3 \%$ resorcinol, $3 \%$ formaldehyde, $0.5 \% \mathrm{KCl}, 0.05 \mathrm{M}$ phosphate, $\mathrm{pH}=7$ ) $\phi=0.186, \mu_{w}=0.70 \mathrm{cp}, 105^{\circ} \mathrm{F}$

\begin{tabular}{|c|c|c|c|c|}
\hline $\begin{array}{c}\text { Flux } \\
\text { (ft/d) }\end{array}$ & $\begin{array}{l}\text { Pressure } \\
\text { Gradient } \\
\text { (osi/ft) }\end{array}$ & $\begin{array}{l}\text { Final } \\
k \\
\text { (md) }\end{array}$ & $F_{\mathrm{rr}}$ & \\
\hline 0.025 & 160 & 0.0174 & 404 & \\
\hline $\begin{array}{l}0.050 \\
0.025\end{array}$ & $\begin{array}{l}283 \\
147\end{array}$ & $\begin{array}{l}0.0195 \\
0.0188\end{array}$ & $\begin{array}{l}358 \\
373\end{array}$ & $\bar{F}_{r r}=365$ \\
\hline $\begin{array}{l}0.100 \\
0.025 \\
0.100\end{array}$ & $\begin{array}{l}534 \\
151 \\
545\end{array}$ & $\begin{array}{l}0.0297 \\
0.0183 \\
0.0203\end{array}$ & $\begin{array}{l}338 \\
382 \\
345\end{array}$ & $\bar{F}_{r r}=355$ \\
\hline $\begin{array}{l}0.202 \\
0.100 \\
0.050 \\
0.025\end{array}$ & $\begin{array}{l}910 \\
511 \\
266 \\
142\end{array}$ & $\begin{array}{l}0.0246 \\
0.0217 \\
0.0208 \\
0.0195\end{array}$ & $\begin{array}{l}285 \\
323 \\
337 \\
360\end{array}$ & $\bar{F}_{r r}=326$ \\
\hline
\end{tabular}


TABLE D4

Results From Brine Injection After Gel Placement in 28-md Berea Sandstone

(Gelant contains $3 \%$ resorcinol, $3 \%$ formaldehyde, $0.5 \% \mathrm{KCl}, 0.05 \mathrm{M}$ phosphate, $\mathrm{pH}=6.5$ )

$\phi=0.185, \mu_{w}=0.70 \mathrm{cp}, 105^{\circ} \mathrm{F}$

\begin{tabular}{|c|c|c|c|}
\hline $\begin{array}{c}\text { Flux } \\
(\text { ft } / d)\end{array}$ & $\begin{array}{l}\text { Pressure } \\
\text { Gradient } \\
\text { (osi/ft) }\end{array}$ & $\begin{array}{l}\text { Final } \\
k \\
(\mathrm{md})\end{array}$ & $\underline{F}_{\mathbf{r}}$ \\
\hline 0.025 & 7.9 & 0.35 & 80 \\
\hline $\begin{array}{l}0.100 \\
0.050 \\
0.025\end{array}$ & $\begin{array}{r}12.6 \\
11.6 \\
8.4\end{array}$ & $\begin{array}{l}0.88 \\
0.48 \\
0.33\end{array}$ & $\begin{array}{l}32 \\
59 \\
85\end{array}$ \\
\hline $\begin{array}{l}0.393 \\
0.201 \\
0.100 \\
0.025\end{array}$ & $\begin{array}{r}34.2 \\
20.7 \\
12.6 \\
9.7\end{array}$ & $\begin{array}{l}1.27 \\
1.08 \\
0.88 \\
0.29\end{array}$ & $\begin{array}{l}22 \\
26 \\
32 \\
98\end{array}$ \\
\hline $\begin{array}{l}1.57 \\
0.393 \\
0.100 \\
0.025\end{array}$ & $\begin{array}{r}130 \\
38.8 \\
15.8 \\
9.0\end{array}$ & $\begin{array}{l}1.33 \\
1.12 \\
0.70 \\
0.31\end{array}$ & $\begin{array}{l}21 \\
25 \\
40 \\
91\end{array}$ \\
\hline $\begin{array}{l}6.28 \\
3.14 \\
0.785 \\
0.393 \\
0.025\end{array}$ & $\begin{array}{r}422 \\
233 \\
62.0 \\
32.6 \\
8.5\end{array}$ & $\begin{array}{l}1.65 \\
1.55 \\
1.40 \\
1.33 \\
0.33\end{array}$ & $\begin{array}{l}17 \\
18 \\
20 \\
21 \\
86\end{array}$ \\
\hline $\begin{array}{l}15.7 \\
6.28 \\
3.14 \\
1.57 \\
0.785 \\
0.393 \\
0.100 \\
0.050 \\
0.025\end{array}$ & $\begin{array}{r}930 \\
397 \\
223 \\
112 \\
58.9 \\
31.1 \\
13.8 \\
9.3 \\
7.3\end{array}$ & $\begin{array}{l}1.87 \\
1.75 \\
1.55 \\
1.48 \\
1.48 \\
1.40 \\
0.80 \\
0.60 \\
0.38\end{array}$ & $\begin{array}{l}15 \\
16 \\
18 \\
19 \\
19 \\
20 \\
35 \\
47 \\
74\end{array}$ \\
\hline
\end{tabular}




\section{TABLE D5}

Results From Brine Injection After Gel Placement in 288-md Berea Sandstone (Gelant contains $3 \%$ resorcinol, $3 \%$ formaldehyde, $0.5 \% \mathrm{KCl}, 0.05 \mathrm{M} \mathrm{NaHCO}_{3}, \mathrm{pH}=6.5$ ) $\phi=0.209, \mu_{w}=0.67 \mathrm{cp}, 105^{\circ} \mathrm{F}$

\begin{tabular}{|c|c|c|c|c|}
\hline $\begin{array}{c}\text { Flux } \\
(\mathrm{ft} / \mathrm{d})\end{array}$ & $\begin{array}{l}\text { Pressure } \\
\text { Gradient } \\
\text { (nsi/ft) }\end{array}$ & $\begin{array}{c}\text { Final } \\
k \\
\text { (md) }\end{array}$ & $\underline{F}_{\mathbf{r}}$ & \\
\hline 0.025 & 10.5 & 0.253 & 1140 & \\
\hline $\begin{array}{l}0.100 \\
0.025\end{array}$ & $\begin{array}{l}29.0 \\
10.7\end{array}$ & $\begin{array}{l}0.364 \\
0.249\end{array}$ & $\begin{array}{r}790 \\
1160\end{array}$ & $F_{\pi}=417 u^{-0.28}$ \\
\hline $\begin{array}{l}0.202 \\
0.100\end{array}$ & $\begin{array}{l}40.8 \\
23.2\end{array}$ & $\begin{array}{l}0.524 \\
0.458\end{array}$ & $\begin{array}{l}550 \\
630\end{array}$ & $F_{\pi}=404 u^{-0.19}$ \\
\hline $\begin{array}{l}0.393 \\
0.100 \\
0.025\end{array}$ & $\begin{array}{r}47.7 \\
17.6 \\
7.2\end{array}$ & $\begin{array}{l}0.87 \\
0.600 \\
0.369\end{array}$ & $\begin{array}{l}330 \\
480 \\
780\end{array}$ & $\varepsilon_{\pi}=242 u^{-0.31}$ \\
\hline $\begin{array}{l}0.785 \\
0.393 \\
0.100\end{array}$ & $\begin{array}{r}69.3 \\
35.4 \\
9.6\end{array}$ & $\begin{array}{l}1.20 \\
1.18 \\
1.11\end{array}$ & $\begin{array}{l}240 \\
245 \\
260\end{array}$ & $\bar{F}_{r}=248$ \\
\hline $\begin{array}{l}3.14 \\
0.785 \\
0.393 \\
0.100\end{array}$ & $\begin{array}{r}169 \\
45.3 \\
24.1 \\
5.3\end{array}$ & $\begin{array}{l}1.98 \\
1.84 \\
1.72 \\
2.00\end{array}$ & $\begin{array}{l}146 \\
157 \\
167 \\
144\end{array}$ & $\bar{F}_{r r}=153$ \\
\hline $\begin{array}{l}15.7 \\
6.28 \\
3.14 \\
1.57 \\
0.785 \\
0.393 \\
0.202\end{array}$ & $\begin{array}{r}629 \\
261 \\
137 \\
76.2 \\
39.2 \\
17.0 \\
9.9\end{array}$ & $\begin{array}{l}2.64 \\
2.55 \\
2.42 \\
2.18 \\
2.12 \\
2.44 \\
2.16\end{array}$ & $\begin{array}{l}109 \\
113 \\
119 \\
132 \\
136 \\
118 \\
133\end{array}$ & $\bar{F}_{r r}=123$ \\
\hline
\end{tabular}


TABLE D6

Results From Brine Injection After Gel Placement in 7.4-md Indiana Limestone (Gelant contains $3 \%$ resorcinol, $3 \%$ formaldehyde, $0.5 \% \mathrm{KCl}, 0.05 \mathrm{M} \mathrm{NaHCO}_{3}, \mathrm{pH}=6.5$ ) $\phi=0.191, \mu_{w}=0.67 \mathrm{cp}, 105^{\circ} \mathrm{F}$

$\begin{array}{ccccc}\begin{array}{c}\text { Flux } \\ (\mathrm{ft} / \mathrm{d})\end{array} & \begin{array}{c}\text { Pressure } \\ \text { Gradient } \\ \text { (esi/ft) }\end{array} & \begin{array}{c}\text { Final } \\ \mathbf{k}\end{array} & \mathrm{F}_{\mathrm{rT}} & \\ 0.393 & 0.8 & \mathrm{md}) & - & \\ 1.57 & 3.1 & 4.91 & 1.5 & \\ 0.785 & 1.9 & 5.27 & 1.4 & \mathrm{~F}_{\mathrm{rr}}=1.5 \mathrm{u}^{-0.07} \\ 3.14 & 6.2 & 4.33 & 1.7 & \\ 6.28 & 11.4 & 5.27 & 1.4 & \\ 15.7 & 28.6 & 5.67 & 1.3 & \\ 3.14 & 6.2 & 5.67 & 1.3 & \\ 0.393 & 0.9 & 5.27 & 1.4 & \\ & & 4.33 & 1.7 & \end{array}$

Table D7

Results From Brine Injection After Gel Placement in 61-md Berea Sandstone (Gelant contains $3 \%$ resorcinol, $3 \%$ formaldehyde, $0.5 \% \mathrm{KCl}, 0.05 \mathrm{M} \mathrm{NaHCO}_{3}, \mathrm{pH}=6.0$ ) $\phi=0.195, \mu_{w}=0.67 \mathrm{cp}, 105^{\circ} \mathrm{F}$

$\begin{array}{ccccc}\begin{array}{c}\text { Flux } \\ (\mathrm{ft} / \mathrm{d})\end{array} & \begin{array}{l}\text { Pressure } \\ \text { Gradient } \\ \text { (osi/ft) }\end{array} & \begin{array}{c}\text { Final } \\ \mathrm{k}\end{array} & \mathrm{F}_{\mathrm{rT}} & \\ 1.57 & 5.2 & 32 & - & \\ 3.14 & 9.8 & 34 & 1.9 & \\ 6.28 & 17.5 & 38 & 1.8 & \mathrm{~F}_{\mathrm{rr}}=2.1 \mathrm{u}^{-0.14} \\ 15.7 & 40.9 & 41 & 1.6 & \\ 6.28 & 16.4 & 41 & 1.5 & \\ 3.14 & 9.8 & 34 & 1.5 & \\ 1.57 & 5.7 & 29 & 1.8 & \end{array}$


TABLE D8

Results From Brine Injection After Gel Placement in 704-md Berea Sandstone (Gelant contains $3 \%$ resorcinol, $3 \%$ formaldehyde, $0.5 \% \mathrm{KCl}, 0.05 \mathrm{M} \mathrm{NaHCO}_{3}, \mathrm{pH}=6.0$ ) $\phi=0.200, \mu_{w}=0.67 \mathrm{cp}, 105^{\circ} \mathrm{F}$

\begin{tabular}{|c|c|c|c|}
\hline $\begin{array}{c}\text { Flux } \\
\text { (ft/d) }\end{array}$ & $\begin{array}{l}\text { Presșure } \\
\text { Gradient } \\
\text { (osi/ft) }\end{array}$ & $\begin{array}{l}\text { Final } \\
k \\
\text { (md) }\end{array}$ & $\mathrm{F}_{\mathrm{rr}}$ \\
\hline $\begin{array}{c}3.14 \\
6.28 \\
15.7 \\
31.4 \\
15.7 \\
6.28 \\
3.14\end{array}$ & $\begin{array}{l}0.9 \\
1.6 \\
4.0 \\
8.1 \\
4.0 \\
1.7 \\
0.9\end{array}$ & $\begin{array}{l}381 \\
408 \\
411 \\
411 \\
414 \\
458 \\
370\end{array}$ & $\begin{array}{l}1.8 \\
1.7 \\
1.7 \\
1.7 \\
1.7 \\
1.8 \\
1.9\end{array}$ \\
\hline
\end{tabular}

TABLE D9

Results From Brine Injection After Gel Placement in 77-md Berea Sandstone (Gelant contains $3 \%$ resorcinol, $3 \%$ formaldehyde, $0.5 \% \mathrm{KCl}, 0.05 \mathrm{M}$ phosphate, $\mathrm{pH}=6$ ) $\phi=0.196, \mu_{\mathrm{w}}=0.70 \mathrm{cp}, 105^{\circ} \mathrm{F}$

$\begin{array}{ccccc}\begin{array}{c}\text { Flux } \\ (\mathrm{ft} / \mathrm{d})\end{array} & \begin{array}{l}\text { Pressure } \\ \text { Gradient } \\ \text { (psi/ft) }\end{array} & \begin{array}{c}\text { Final } \\ \mathrm{k}\end{array} & \mathrm{F}_{\mathrm{rr}} & \\ 1.57 & 3.2 & \mathrm{md}) & - & \\ 3.14 & 5.4 & 55 & 1.4 & \\ 1.57 & 3.6 & 64 & 1.2 & \\ 6.28 & 10.8 & 48 & 1.6 & -\bar{F}_{\mathrm{rr}}=1.3 \\ 3.14 & 5.9 & 64 & 1.2 & \\ 15.7 & 27.1 & 59 & 1.3 & \\ 6.28 & 11.7 & 64 & 1.2 & \end{array}$

TABLE D10

Results From Brine Injection After Gel Placement in 573-md Berea Sandstone (Gelant contains $3 \%$ resorcinol, $3 \%$ formaldehyde, $0.5 \% \mathrm{KCl}, 0.05 \mathrm{M}$ acetate, $\mathrm{pH}=5$ ) $\phi=0.207, \mu_{\mathrm{w}}=0.67 \mathrm{cp}, 105^{\circ} \mathrm{F}$

$\begin{array}{ccccc}\begin{array}{c}\text { Flux } \\ (\mathrm{ft} / \mathrm{d})\end{array} & \begin{array}{l}\text { Pressure } \\ \text { Gradient } \\ \text { (esi/ft) }\end{array} & \begin{array}{c}\text { Final } \\ \mathrm{k}\end{array} & \mathrm{F}_{\mathrm{rr}} & \\ 15.7 & 2.9 & \text { (md) } & - & \\ 31.4 & 5.2 & 573 & 1.0 & \overline{\mathrm{F}}_{\mathrm{rr}}=1.0\end{array}$


APPENDIX E

DATA FROM VISCOUS FINGERING EXPERIMENTS 


\section{APPENDIX E \\ DATA FROM VISCOUS FINGERING EXPERIMENTS}

See Fig. 40 and Nomenclature for definitions of $r_{p}, r_{f o}, r_{f l}$, and $r_{v f}$.

Table E1

Results Using 11-cp Ethanediol and 1-cp Water

\begin{tabular}{|c|c|c|c|c|c|c|c|c|c|}
\hline Run & $\begin{array}{l}\text { Bead } \\
\text { Pack }\end{array}$ & $\begin{array}{c}r_{p} \\
(\mathrm{~cm}) \\
\end{array}$ & $\begin{array}{l}\mathrm{r}_{\text {fo }} \\
(\mathrm{cm})\end{array}$ & $\begin{array}{l}r_{\mathfrak{f}} \\
(\mathrm{cm})\end{array}$ & $\begin{array}{l}r_{\mathrm{vf}} \\
(\mathrm{cm})\end{array}$ & $\begin{array}{l}\text { No. of } \\
\text { fingers }\end{array}$ & $\begin{array}{l}1^{\text {st }} \text { Break- } \\
\text { through }\end{array}$ & $\begin{array}{c}r_{v f} / \\
\left(r_{f o}-r_{n}\right)\end{array}$ & $\begin{array}{c}\left(r_{v}+r_{f i}\right) \\
r_{n} \\
\end{array}$ \\
\hline 1 & $\begin{array}{l}1 \\
2\end{array}$ & $\begin{array}{l}36.8 \\
18.5\end{array}$ & $\begin{array}{l}48.0 \\
20.5\end{array}$ & $\begin{array}{l}27.0 \\
11.0\end{array}$ & $\begin{array}{c}21.0 \\
7.0\end{array}$ & $\begin{array}{l}7 \\
4\end{array}$ & $X$ & $\begin{array}{l}1.00 \\
0.74\end{array}$ & $\begin{array}{l}1.78 \\
1.64\end{array}$ \\
\hline 2 & $\begin{array}{l}1 \\
2\end{array}$ & $\begin{array}{l}37.4 \\
15.0\end{array}$ & $\begin{array}{l}45.0 \\
16.5\end{array}$ & $\begin{array}{l}29.0 \\
11.3\end{array}$ & $\begin{array}{c}16.0 \\
3.5\end{array}$ & $\begin{array}{c}7 \\
20\end{array}$ & $X$ & $\begin{array}{l}1.00 \\
0.67\end{array}$ & $\begin{array}{l}1.55 \\
1.31\end{array}$ \\
\hline 3 & $\begin{array}{l}1 \\
2\end{array}$ & $\begin{array}{l}36.8 \\
13.0\end{array}$ & $\begin{array}{l}44.2 \\
15.5\end{array}$ & $\begin{array}{c}22.0 \\
6.2\end{array}$ & $\begin{array}{c}18.0 \\
9.3\end{array}$ & $\begin{array}{c}4 \\
12\end{array}$ & $X$ & $\begin{array}{l}0.81 \\
1.00\end{array}$ & $\begin{array}{l}1.82 \\
2.50\end{array}$ \\
\hline 4 & $\begin{array}{l}1 \\
2\end{array}$ & $\begin{array}{l}34.5 \\
13.2\end{array}$ & $\begin{array}{l}39.0 \\
15.0\end{array}$ & $\begin{array}{c}17.0 \\
6.2\end{array}$ & $\begin{array}{c}18.0 \\
8.8\end{array}$ & $\begin{array}{c}4 \\
11\end{array}$ & $X$ & $\begin{array}{l}0.82 \\
1.00\end{array}$ & $\begin{array}{l}2.06 \\
2.42\end{array}$ \\
\hline 5 & $\begin{array}{l}1 \\
2\end{array}$ & $\begin{array}{l}33.9 \\
15.4\end{array}$ & $\begin{array}{l}39.0 \\
16.8\end{array}$ & $\begin{array}{c}16.4 \\
9.4\end{array}$ & $\begin{array}{c}22.6 \\
3.8\end{array}$ & $\begin{array}{c}6 \\
22\end{array}$ & $\mathbf{X}$ & $\begin{array}{l}1.00 \\
0.51\end{array}$ & $\begin{array}{l}2.38 \\
1.40\end{array}$ \\
\hline 6 & $\frac{1}{2}$ & $\begin{array}{l}34.2 \\
14.0\end{array}$ & $\begin{array}{l}39.8 \\
15.0\end{array}$ & $\begin{array}{l}16.3 \\
11.0\end{array}$ & $\begin{array}{c}23.5 \\
2.5\end{array}$ & $\begin{array}{c}4 \\
21\end{array}$ & $X$ & $\begin{array}{l}1.00 \\
0.63\end{array}$ & $\begin{array}{l}2.44 \\
1.23\end{array}$ \\
\hline 7 & $\begin{array}{l}1 \\
2\end{array}$ & $\begin{array}{l}34.2 \\
14.7\end{array}$ & $\begin{array}{l}40.0 \\
16.0\end{array}$ & $\begin{array}{l}18.5 \\
12.0\end{array}$ & $\begin{array}{c}21.5 \\
2.0\end{array}$ & $\begin{array}{c}6 \\
27\end{array}$ & $X$ & $\begin{array}{l}1.00 \\
0.50\end{array}$ & $\begin{array}{l}2.16 \\
1.17\end{array}$ \\
\hline 8 & $\begin{array}{l}1 \\
2\end{array}$ & $\begin{array}{l}34.0 \\
13.0\end{array}$ & $\begin{array}{l}40.0 \\
14.6\end{array}$ & $\begin{array}{l}15.5 \\
12.0\end{array}$ & $\begin{array}{c}17.3 \\
2.6\end{array}$ & $\begin{array}{c}5 \\
25\end{array}$ & $\mathrm{X}$ & $\begin{array}{l}0.71 \\
i .00\end{array}$ & $\begin{array}{l}2.12 \\
1.22\end{array}$ \\
\hline 9 & $\begin{array}{l}1 \\
2\end{array}$ & $\begin{array}{l}35.0 \\
11.7\end{array}$ & $\begin{array}{l}41.2 \\
14.0\end{array}$ & $\begin{array}{c}18.5 \\
7.5\end{array}$ & $\begin{array}{c}22.7 \\
2.0\end{array}$ & $\begin{array}{c}4 \\
20\end{array}$ & $X$ & $\begin{array}{l}1.00 \\
0.31\end{array}$ & $\begin{array}{l}2.22 \\
1.27\end{array}$ \\
\hline 10 & $\begin{array}{l}1 \\
2\end{array}$ & $\begin{array}{l}35.3 \\
12.3\end{array}$ & $\begin{array}{l}42.5 \\
14.5\end{array}$ & $\begin{array}{c}19.0 \\
9.1\end{array}$ & $\begin{array}{c}23.5 \\
3.9\end{array}$ & $\begin{array}{c}7 \\
15\end{array}$ & $X$ & $\begin{array}{l}1.00 \\
0.72\end{array}$ & $\begin{array}{l}2.24 \\
1.43\end{array}$ \\
\hline ave. & $\begin{array}{l}\overline{1} \\
2\end{array}$ & $\begin{array}{l}\overline{35.2} \\
14.1\end{array}$ & $\begin{array}{l}\overline{41.9} \\
15.8\end{array}$ & $\begin{array}{c}\overline{19.9} \\
9.6\end{array}$ & $\begin{array}{c}\overline{20.4} \\
4.5\end{array}$ & $\begin{array}{c}5 \\
18\end{array}$ & & $\begin{array}{l}\overline{0.93} \\
0.71\end{array}$ & $\begin{array}{l}\overline{2.08} \\
1.56\end{array}$ \\
\hline
\end{tabular}


Table E2

Results Using 2000-ppm Xanthan and 1-cp Water

\begin{tabular}{|c|c|c|c|c|c|c|c|c|c|}
\hline Run & $\begin{array}{l}\text { Bead } \\
\text { Pack }\end{array}$ & $\stackrel{r_{p}}{(\mathrm{~cm})}$ & $\begin{array}{l}{ }^{f_{\text {fo }}} \\
\text { (cm) }\end{array}$ & $\begin{array}{l}r_{\mathrm{ft}} \\
\mathrm{cm})\end{array}$ & $\begin{array}{l}r_{\mathrm{vf}} \\
(\mathrm{cm})\end{array}$ & $\begin{array}{l}\text { No. of } \\
\text { fingers }\end{array}$ & $\begin{array}{l}1^{\text {st }} \text { Break- } \\
\text { through }\end{array}$ & $\begin{array}{c}r_{v f} / \\
\left(r_{r_{0}}-r_{n}\right)\end{array}$ & $\begin{array}{c}\left(r_{\mathrm{ve}}+r_{\mathrm{r}}\right) \\
\mathrm{r}_{\mathrm{r}} \\
\end{array}$ \\
\hline 1 & $\begin{array}{l}1 \\
2\end{array}$ & $\begin{array}{l}36.0 \\
14.0\end{array}$ & $\begin{array}{l}39.5 \\
14.1\end{array}$ & $\begin{array}{l}4.0 \\
4.1\end{array}$ & $\begin{array}{c}33.5 \\
9.9\end{array}$ & $\begin{array}{l}10 \\
10\end{array}$ & $X$ & $\begin{array}{l}0.94 \\
1.00\end{array}$ & $\begin{array}{l}9.38 \\
3.41\end{array}$ \\
\hline 2 & $\begin{array}{l}1 \\
2\end{array}$ & $\begin{array}{l}35.5 \\
12.3\end{array}$ & $\begin{array}{l}36.1 \\
13.2\end{array}$ & $\begin{array}{l}7.7 \\
5.0\end{array}$ & $\begin{array}{c}28.4 \\
6.8\end{array}$ & $\begin{array}{c}8 \\
11\end{array}$ & $X$ & $\begin{array}{l}1.00 \\
0.83\end{array}$ & $\begin{array}{l}4.69 \\
2.36\end{array}$ \\
\hline 3 & $\begin{array}{l}1 \\
2\end{array}$ & $\begin{array}{l}35.6 \\
12.0\end{array}$ & $\begin{array}{l}39.0 \\
12.5\end{array}$ & $\begin{array}{l}5.5 \\
3.5\end{array}$ & $\begin{array}{c}33.5 \\
8.0\end{array}$ & $\begin{array}{l}11 \\
10\end{array}$ & $X$ & $\begin{array}{l}1.00 \\
0.89\end{array}$ & $\begin{array}{l}7.09 \\
3.29\end{array}$ \\
\hline 4 & $\begin{array}{l}1 \\
2\end{array}$ & $\begin{array}{l}36.0 \\
11.2\end{array}$ & $\begin{array}{l}39.1 \\
12.0\end{array}$ & $\begin{array}{l}5.5 \\
4.3\end{array}$ & $\begin{array}{c}30.7 \\
7.7\end{array}$ & $\begin{array}{l}14 \\
15\end{array}$ & $X$ & $\begin{array}{l}0.91 \\
1.00\end{array}$ & $\begin{array}{l}6.58 \\
2.79\end{array}$ \\
\hline 5 & $\begin{array}{l}1 \\
2\end{array}$ & $\begin{array}{l}35.9 \\
11.5\end{array}$ & $\begin{array}{l}39.8 \\
12.0\end{array}$ & $\begin{array}{l}6.4 \\
4.5\end{array}$ & $\begin{array}{c}33.4 \\
6.4\end{array}$ & $\begin{array}{l}9 \\
9\end{array}$ & $X$ & $\begin{array}{l}1.00 \\
0.85\end{array}$ & $\begin{array}{l}6.22 \\
2.42\end{array}$ \\
\hline 6 & $\begin{array}{l}1 \\
2\end{array}$ & $\begin{array}{l}35.6 \\
12.0\end{array}$ & $\begin{array}{l}38.5 \\
12.5\end{array}$ & $\begin{array}{l}9.0 \\
4.3\end{array}$ & $\begin{array}{c}29.5 \\
7.1\end{array}$ & $\begin{array}{l}15 \\
15\end{array}$ & $X$ & $\begin{array}{l}1.00 \\
0.87\end{array}$ & $\begin{array}{l}4.28 \\
2.65\end{array}$ \\
\hline 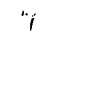 & $\begin{array}{l}1 \\
2\end{array}$ & $\begin{array}{l}35.8 \\
11.6\end{array}$ & $\begin{array}{l}38.4 \\
12.0\end{array}$ & $\begin{array}{l}8.0 \\
4.5\end{array}$ & $\begin{array}{c}30.4 \\
5.9\end{array}$ & $\begin{array}{l}20 \\
27\end{array}$ & $X$ & $\begin{array}{l}1.00 \\
0.79\end{array}$ & $\begin{array}{l}4.80 \\
2.31\end{array}$ \\
\hline 8 & $\begin{array}{l}1 \\
2\end{array}$ & $\begin{array}{l}35.6 \\
11.9\end{array}$ & $\begin{array}{l}38.4 \\
12.5\end{array}$ & $\begin{array}{c}10.8 \\
4.5\end{array}$ & $\begin{array}{c}27.6 \\
6.3\end{array}$ & $\begin{array}{l}10 \\
19\end{array}$ & $X$ & $\begin{array}{l}1.00 \\
0.79\end{array}$ & $\begin{array}{l}3.56 \\
2.40\end{array}$ \\
\hline 9 & $\begin{array}{l}1 \\
2\end{array}$ & $\begin{array}{l}36.0 \\
11.5\end{array}$ & $\begin{array}{l}39.0 \\
12.2\end{array}$ & $\begin{array}{c}11.0 \\
4.0\end{array}$ & $\begin{array}{c}28.0 \\
5.5\end{array}$ & $\begin{array}{l}15 \\
20\end{array}$ & $X$ & $\begin{array}{l}1.00 \\
0.67\end{array}$ & $\begin{array}{l}3.55 \\
2.38\end{array}$ \\
\hline 10 & $\begin{array}{l}1 \\
2\end{array}$ & $\begin{array}{l}36.0 \\
11.1\end{array}$ & $\begin{array}{l}39.5 \\
11.8\end{array}$ & $\begin{array}{c}10.0 \\
5.0\end{array}$ & $\begin{array}{r}29.5 \\
5.4\end{array}$ & $\begin{array}{c}9 \\
11\end{array}$ & $X$ & $\begin{array}{l}1.00 \\
0.79\end{array}$ & $\begin{array}{l}3.95 \\
2.08\end{array}$ \\
\hline 11 & $\begin{array}{l}1 \\
2\end{array}$ & $\begin{array}{l}36.0 \\
10.3\end{array}$ & $\begin{array}{l}39.0 \\
10.8\end{array}$ & $\begin{array}{l}8.5 \\
3.5\end{array}$ & $\begin{array}{c}28.5 \\
7.3\end{array}$ & $\begin{array}{l}12 \\
18\end{array}$ & $X$ & $\begin{array}{l}0.93 \\
1.00\end{array}$ & $\begin{array}{l}4.35 \\
3.09\end{array}$ \\
\hline 12 & $\begin{array}{l}1 \\
2\end{array}$ & $\begin{array}{l}36.3 \\
11.0\end{array}$ & $\begin{array}{l}39.0 \\
11.4\end{array}$ & $\begin{array}{c}10.0 \\
4.5\end{array}$ & $\begin{array}{c}29.0 \\
5.9\end{array}$ & $\begin{array}{l}12 \\
12\end{array}$ & $X$ & $\begin{array}{l}1.00 \\
0.86\end{array}$ & $\begin{array}{l}3.90 \\
2.31\end{array}$ \\
\hline ave. & $\begin{array}{l}1 \\
2\end{array}$ & $\begin{array}{l}35.8 \\
11.6\end{array}$ & $\begin{array}{l}38.8 \\
12.2\end{array}$ & $\begin{array}{l}8.0 \\
4.3\end{array}$ & $\begin{array}{c}\overline{30.2} \\
6.9\end{array}$ & $\begin{array}{l}\overline{12} \\
15\end{array}$ & & $\begin{array}{l}\overline{0.98} \\
0.86\end{array}$ & $\begin{array}{l}\overline{5.20} \\
2.62\end{array}$ \\
\hline
\end{tabular}


Table E3

Results Using 2000-ppm HPAM and 1-cp Water

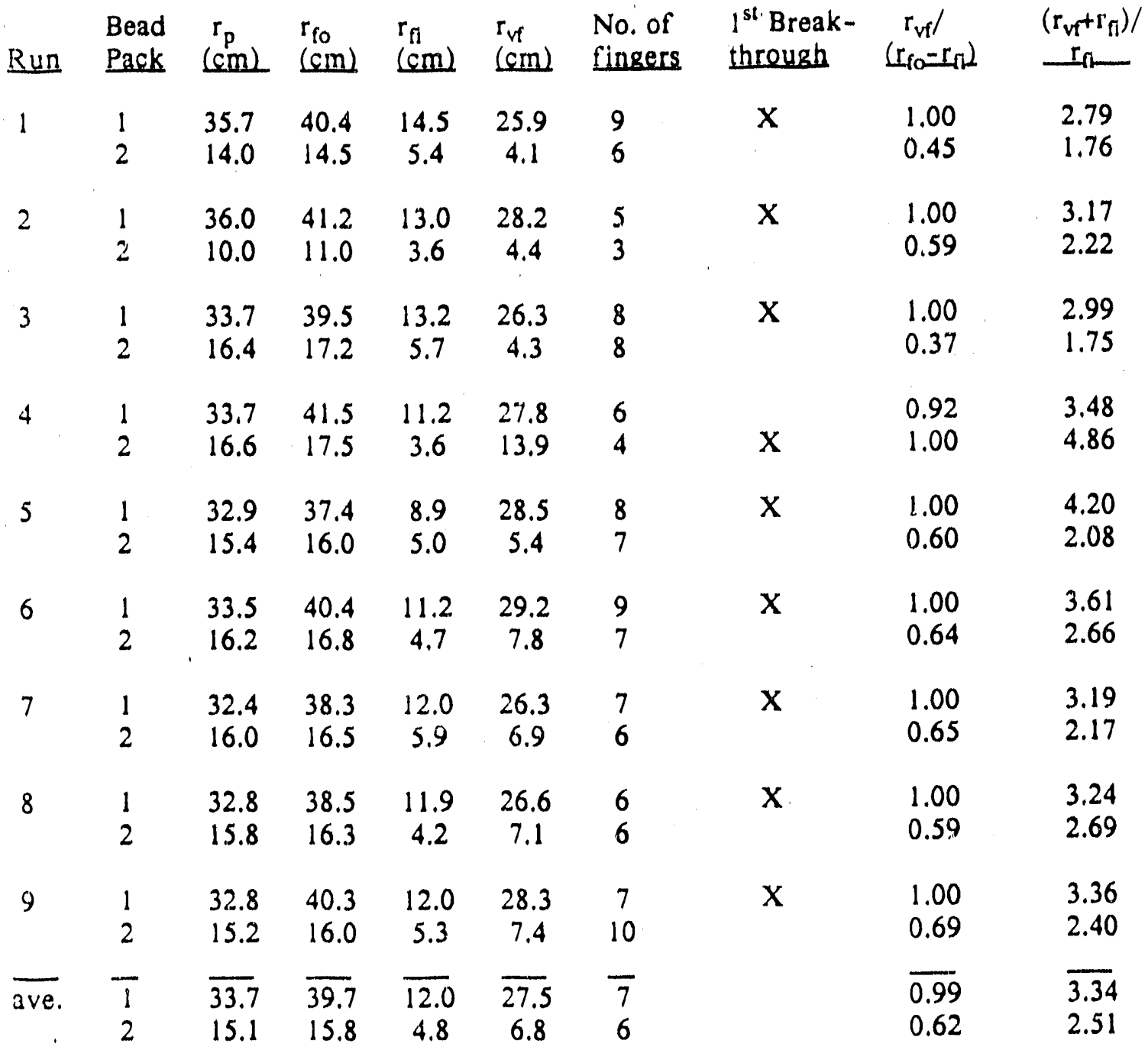


APPENDIX F

FORTRAN CODING FOR THEORETICAL MODEL: RADIAL FL,OW 
C

PROGRAM RADIAL

C PROGRAM INFORMATION:

C

C BASED UPON THE THEORETICAL MODEL DEVELOPED IN THIS PAPER, A

C FORTRAN PRCGRAM WAS DEVELOPED TO DETERMINE THE DEGREE OF

C PENETRATION OF GELANTS INTO OIL-PRODUCTIVE STRATA AS WELL AS

C INTO WATER-SOURCE ZONES. THE TECHNIQUES USED IN THIS PROGRAM

C TO CALCULATE FRONTAL SATURATIONS, SATURATION PROFILES, AND

C AVER AGE SATURATIONS CAN BE FOUND IN REFS. 103, 104, AND 105.

$\mathrm{C}$

C PROGRAM LIMITATIONS:

C IN ADDITION TO THE ASSUMPTIONS MADE IN DEVELOPING THE

C THEORETICAL MODEL, THE LIIATATIONS OF THIS FORTRAN PROGRAM

C ARE LISTED BELOW:

C 1. THIS PROGRAM DEALS WITH RADIAL CASES ONLY.

C 2. IT IS ASSUMED IN THIS PROGRAM THAT, IN ANY LAYER, THE

C OIL BANK (FR>1) OR THE WATER FRONT (FR=1) DOESN'T

$\mathrm{C}$

$\mathrm{C}$

C

C

C

C

C

$\mathrm{C}$

C

C

C DEFINITION OF VARIABLES:

C

C

AINF! $=$ FIRST GUESS OF THE WATER SATURATION AT THE INFLECTION POINT OF THE FRACTIONAL FLOW CURVE (LAYER 1)

AINF2 $=$ SECOND GUESS OF THE WATER SATURATION AT THE INFLECTION POINT OF THE FRACTIONAL FLOW CURVE (LAYER i)

AINFII $=$ FIRST GUESS OF THE WATER SATURATION AT THE INFLECTION POINT OF THE FRACTIONAL FLOW CURVE (L A YER I)

C

C

C

C

C

C

$\begin{aligned} & \text { AINFI2 }= \text { SECOND GUESS OF THE WATER SATURATION AT THE } \\ & \text { INFLELTION POINT OF THE FRACTIONAL FLOW CURVE }\end{aligned}$ (LAYER I)

AK $=$ PERMEABILITY, MD

$A K R O=$ OIL RELATIVE PERMEABILITY

$A K R O O=$ END-POINT OIL RELATIVE PERMEABILITY

$A K R W=$ WATER RELATIVE PERMEABILITY

$A K R W O=$ END-POINT WATER RELATIVE PERMEABILITY

$A L_{i}=$

THE FIRST INTEGRATION TERM (GELANT FRONT) ON THE LEFT-HAND-SIDE OF THE THEORETICAL EQUATION (LAYER?

C AMO $=$ WATER-OIL MOBILITY 


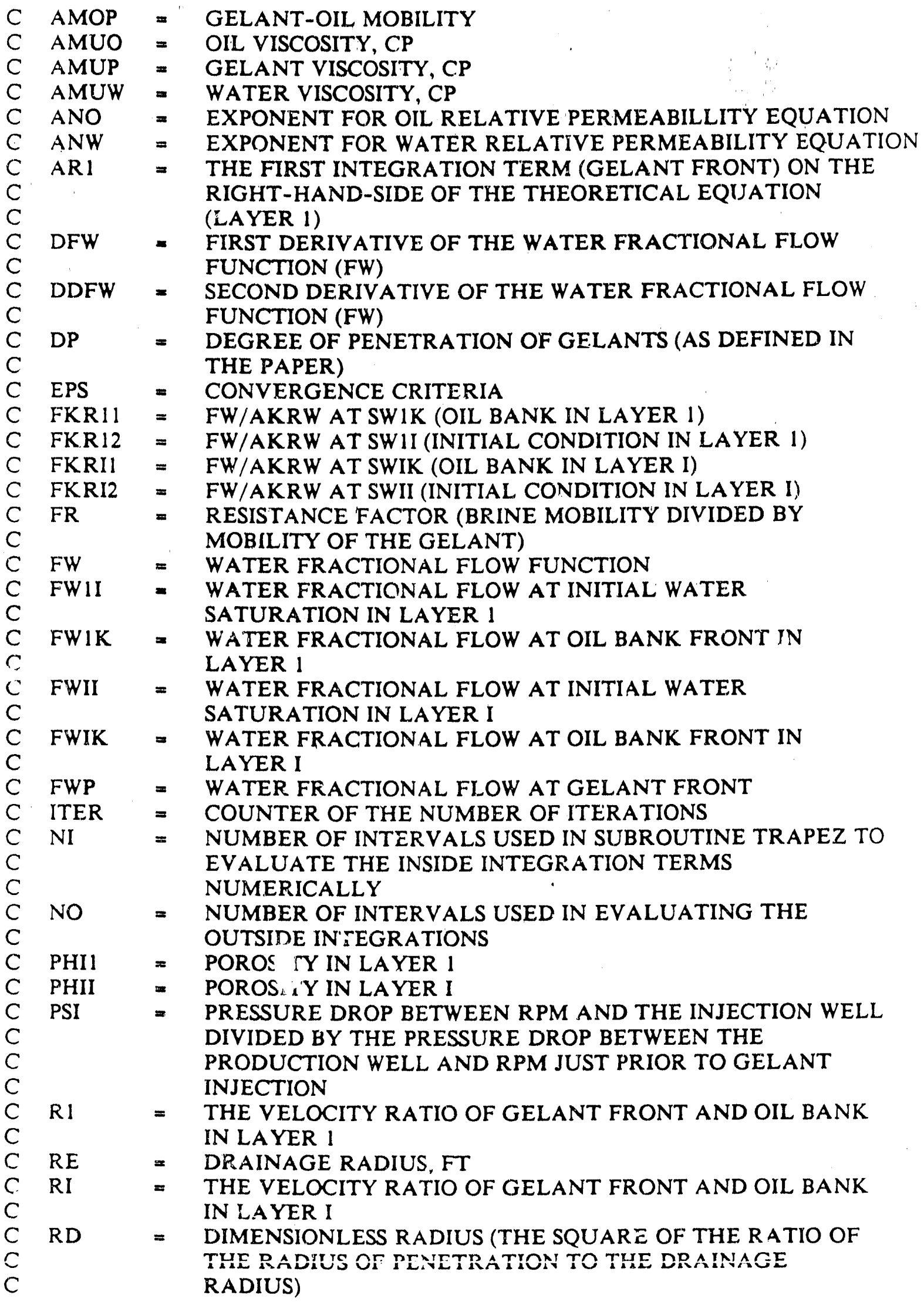




\begin{tabular}{|c|c|c|c|}
\hline $\begin{array}{l}\mathrm{C} \\
\mathrm{C}\end{array}$ & RDI & $=$ & $\begin{array}{l}\text { DIMENSIONLESS RADIUS OF GELANT PENETRATION INTO } \\
\text { LAYER } 1\end{array}$ \\
\hline 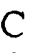 & RDO & $=$ & DIMENSIONLESS WELLBORE RADIUS \\
\hline 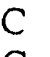 & RDPM & $=$ & A DIMENSIONLESS REFERENCE DISTANCE FROM THE \\
\hline 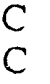 & & & $\begin{array}{l}\text { WELLBORE BEYOND WHICH THE GEL TREATMENT HAS NO } \\
\text { EFFECT ON FLUID SATURATION }\end{array}$ \\
\hline $\mathrm{C}$ & $\mathrm{RC}$ & $=$ & WELLBORE RADIUS, FT \\
\hline $\mathrm{C}$ & RPI & $=$ & RADIUS OF GELANT PENETRATION INTO LA YER 1, FT \\
\hline C & RPM & $=$ & $\begin{array}{l}\text { A REFERENCE DISTANCE FROM THE WELLBORE BEYOND } \\
\text { WHICH THE GEL TREATMENT HAS NO EFFECT ON FLUID }\end{array}$ \\
\hline 0 & & & $\begin{array}{l}\text { WHICH THE GEL TREA IMENI HA } \\
\text { SATURATION, FT }\end{array}$ \\
\hline C & RRI & $=$ & $\begin{array}{l}\text { THE VELOCITY RATIO OF GELANT FRONT AND WATER } \\
\text { FRONT IN LAYER } 1\end{array}$ \\
\hline $\begin{array}{l}\mathrm{C} \\
\mathrm{C}\end{array}$ & RRI & $=$ & $\begin{array}{l}\text { THE VELOCITY RATIO OF GELANT FRONT AND WATER } \\
\text { FRONT IN LAYER I }\end{array}$ \\
\hline 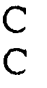 & SHKII & $=$ & $\begin{array}{l}\text { FIRST GUESS OF THE SATURATION AT THE GELANT } \\
\text { FRONT (LAYER 1) }\end{array}$ \\
\hline C & SHK 12 & $=$ & FRONT (LAYER 1) \\
\hline C & SHKXII & & $\begin{array}{l}\text { FRONT (LAYER 1) } \\
\text { FIRST GUESS OF THE SATURATION AT THE }\end{array}$ \\
\hline 1 & & & SATURATION AT THE GELANT \\
\hline $\begin{array}{l}C \\
C\end{array}$ & SHKI12 & $=$ & $\begin{array}{l}\text { SECOND GUESS OF THE SATURATION AT THE GELANT } \\
\text { FRONT (LA YER I) }\end{array}$ \\
\hline $\begin{array}{l}\mathrm{C} \\
\mathrm{C}\end{array}$ & SHK 21 & $=$ & $\begin{array}{l}\text { FIRST GUESS OF THE SATURATION AT THE OIL BANK } \\
\text { (LAYER 1) }\end{array}$ \\
\hline $\begin{array}{l}\mathrm{C} \\
\mathrm{C}\end{array}$ & SHK 22 & $=$ & $\begin{array}{l}\text { SECOND GUESS OF THE SATURATION AT THE OIL BANK } \\
\text { (LAYER 1) }\end{array}$ \\
\hline $\begin{array}{l}\mathrm{C} \\
\mathrm{C}\end{array}$ & SHKI2I & $=$ & $\begin{array}{l}\text { FIRST GUESS OF THE SATURATION AT THE OIL BANK } \\
\text { (LAYER I) }\end{array}$ \\
\hline C & SHKI22 & $=$ & $\begin{array}{l}\text { SECOND GUESS OF THE SATURATION AT THE OIL BANK } \\
\text { (LAYER I) }\end{array}$ \\
\hline $\mathrm{C}$ & SOR & $=$ & RESIDUAL OIL SATURATION \\
\hline C & $\begin{array}{l}\text { SWAVG } \\
\text { SWCTY,S }\end{array}$ & $=$ & AVERAGE WATER SATURATION BEHIND GELANT FRONT \\
\hline C & SWCTI,S & 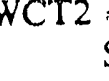 & $\begin{array}{l}=\text { INITIAL GUESSES OF THE CRITICAL WATER } \\
\text { SATURATION }\end{array}$ \\
\hline C & SWCT & $=$ & $\begin{array}{l}\text { SATURATION } \\
\text { CRITICAL WATER SATURATION }\end{array}$ \\
\hline & SWIF & $=$ & $\begin{array}{l}\text { CRITICAL WATER SATURATION } \\
\text { WATER SATURATION AT THE WATER FRONT (FR=1, }\end{array}$ \\
\hline C & SWII & $=$ & 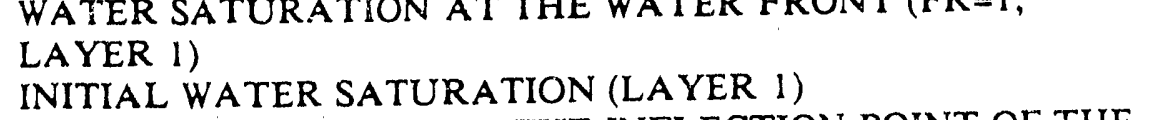 \\
\hline C & SWIINF & $=$ & $\begin{array}{l}\text { INITIAL WATER SATURATION (LA YER 1) } \\
\text { WATER SATURATION AT THE INFLECTION POINT C }\end{array}$ \\
\hline C & SWIK & & $\begin{array}{l}\text { WATER SATURATION AT THE INFLECTION POINI UF } \\
\text { FRACTIONAL FLOW CURVE (LAYER 1) } \\
\text { WATER SATURATION AT THE OIL BANK (LAYER 1) }\end{array}$ \\
\hline 0 & SWIPF & $=$ & $\begin{array}{l}\text { URATION AT THE OIL BANK (LAYER 1) } \\
\text { URATION AT THE GELANT FRONT (LAYE }\end{array}$ \\
\hline C & SWIF & $=$ & $\begin{array}{l}\text { WATER SATURATION AT THE GELANT FRONT (LAYE } \\
\text { WATER SATURATION AT THE WATER FRONT (FR=1, }\end{array}$ \\
\hline C & SWII & $=$ & $\begin{array}{l}\text { LAYER I) } \\
\text { INITIAL WATER SATURATION (LAYER I) }\end{array}$ \\
\hline C & SWIINF & $\begin{array}{l}= \\
=\end{array}$ & $\begin{array}{l}\text { INITIAL WATER SATURATION (LAYER I) } \\
\text { WATER SATURATION AT THE INFLECTION POINT OF THE }\end{array}$ \\
\hline C & & $=$ & $\begin{array}{l}\text { WATER SATURATION AT THE INFLECTION POINT OF } \\
\text { FRACTIONAL FLOW CURVE (LAYER I) } \\
\text { WATER SATURATION AT THE OIL BANK (LAYER I) }\end{array}$ \\
\hline C & $\begin{array}{l}\text { SWR } \\
\text { TD }\end{array}$ & $=$ & $\begin{array}{l}\text { WATER SATURATION AT THE GE } \\
\text { RESIDUAL WATER SATURATION }\end{array}$ \\
\hline & & $=$ & $\begin{array}{l}\text { RESIDUAL WATER SATURATION } \\
\text { DIMENSIONLESS TIME DEFINED AS FRACTION OF A PO }\end{array}$ \\
\hline
\end{tabular}




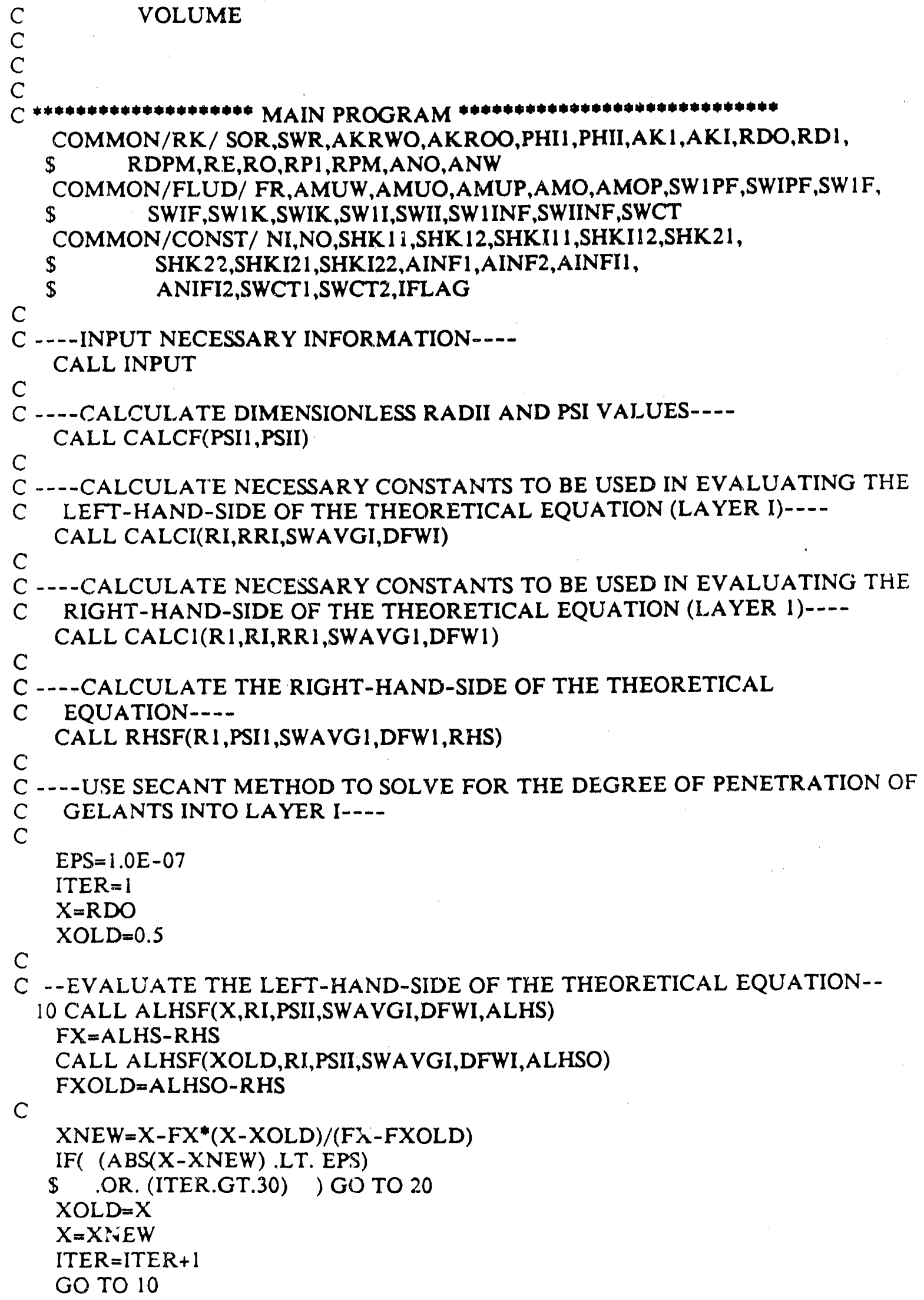




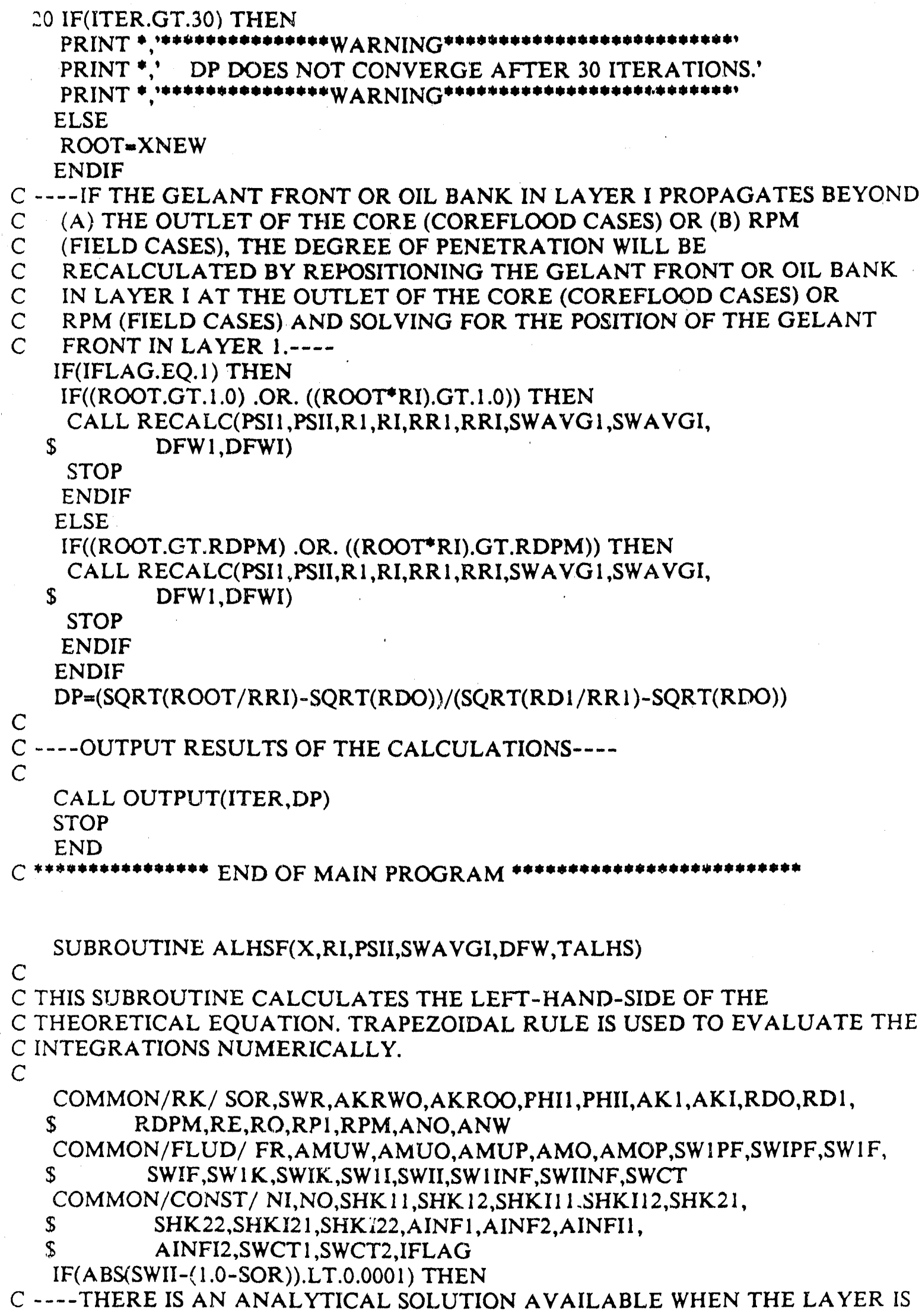


C WATERED-OUT.----

TALHS=(1.0-SOR)/AKRWO*((FR-1.0)*((X*ALOG(X)-X)-

$\$$ (RDO*ALOG(RDO)-RDO))-(PSII+FR)*ALOG(RDO)*(X-RDO)+

$\$$ (PSII+1.0)*ALOG(RDPM)*(X-RDO))

ELSE

IF(ABS(FR-1.0), :.T.0.0001) THEN

C -.--THERE IS NO GII 3 A NNK WHEN FR=1.0...--

CALL FWKRW(

FKRII=FKRI2

IF(SWII.LT.SWIINF) THEN

SWIPFC=SWIF

ELSE

IF(SWII.GT.SWIPF) THEN

SWIPFC $=$ SWII

ELSE

SWIPFC $=$ SWII

E.NDIF

ENDIF

ELSE

C -.-CALCULATE FW/KRW FOR BOTH OIL BANK AND INITIAL CONDITION

C WHEN FR>1.0....-

CALL FWKRW(AMO,SWIK,FKRII)

CALL FWKRW(AMO,SWII,FKRI2)

SWIPFC=SWIPF

ENDIF

C----USE TRAPEZOIDAL RULE TO EVALUATE THE INTEGRATIONS

C NUMERICALL,Y.----

$\mathrm{H}=(\mathrm{X}-\mathrm{RDO}) / \mathrm{NO}$

SUM $=0.0$

DO $200 \mathrm{I}=1$, NO- 1

$\mathrm{T}=\mathrm{RDO}+\mathrm{I}^{*} \mathrm{H}$

TD $=T / D F W$

CALL TRAPEZ(RDO,T,SWIPFC,NI,AMOP,TD,AL1)

IF(RI.LT.0.0001) THEN

$\mathrm{RIT}=0.0$

ELSE

$R I T=A L O G\left(R I^{*} T\right)$

ENDIF

ALHS $=(A L 1 * F R+F K R I 1 *(R I T-A L O G(T))+F K R I 2 *(A L O G(R D P M)$

$\left.\left.\left.\$ \quad-R I T+P S I I^{*}(A L O G(R D P M)-A L O G(R D O))\right)\right)\right)^{*}$ SWAVGI

SUM $=S U M+A L H S$

200 CONTINUE

$T D=X / D F W$

CALL TRAPEZ(RDO,X,SWIPFC,NI,AMOP,TD,AL1)

IF(RI.LT.0.0001) THEN

$\mathrm{RIX}=0.0$

RIRDO $=0.0$

ELSE

$R I X=A L O G\left(R I^{*} X\right)$

RIRDO=ALOG $\left(R I^{*} R D O\right)$

ENDIF

$A L H S B=(A L I * F R+F K R I 1 *(R I X-A L O G(X))+F K R I 2 *(A L O G(R D P M)$ 
$\left.\left.\$ \quad-R I X+P S I I^{*}(A L O G(R D P M)-A L O G(R D O))\right)\right) *$ SWAVGI

ALHSA $=($ FKRII $($ RIRDO-ALOG(RDO))+FKRI2* (ALOG(RDPM)

-RIRDO+PSII*(ALOG(RDPM)-ALOG(RDO))))*SWAVGI

TALHS $=(H / 2.0)^{*}(A L H S B+2.0 *$ SUM+ALHSA $)$

ENDIF

RETURN

END

SUBROUTINE CALCF(PSII,PSII)

$\mathrm{C}$

C THIS SUBROUTINE CALCULATES DIMENSIONLESS CONSTANTS AND PSI

C VALUES.

$\mathrm{C}$

COMMON/RK/ SOR,SWR,AKRWO,AKROO,PHII,PHII,AKI,AKI,RDO,RDI, \$ RDPM,RE,RO,RPI,RPM,ANO,ANW

COMMON/FLUD/FR,AMUW,AMUO,AMUP,AMO,AMOP,SWIPF,SWIPF,SWIF,

$\$$ SWIF,SWIK,SWIK,SW1I,SWII,SWIINF,SWIINF,SWCT

COMMON/CONST/ NI,NO,SHK 11 ,SHK 12,SHKI11,SHK112,SHK21,

$\$$ SHK22,SHKI2:,SHKI22, AINF1, AINF2,AINFI1,

$\$$ ANIFI2,SWCT1,SWCT2,IFLAG

IF( IFLAG.EQ.2) THEN

C -..-CALCULATE DIMENSIONLESS CONSTANTS FOR FIELD CASES

C (5-SPOT)----

$\mathrm{D}=\mathrm{RE} * 3.14159 * 0.5$

$R D O=(R O / D) * 2.0$

$\mathrm{RDI}=(\mathrm{RPI} / \mathrm{D})^{* *} 2.0$

$R D P M=(R P M / D) * 2.0$

ELSE

C -.--SET PSI VALUES TO ZERO AND CALCULATE THE DIMENSIONLESS

C CONSTANTS FOR COREFLOOD CASES.-.--

$\mathrm{RDO}=(\mathrm{RO} / \mathrm{RE}) * * 2.0$

$R D P M=(R P M / R E)^{* * 2.0}$

PSI $1=0.0$

PSII $=0.0$

RETURN

ENDIF

C ---CALCULATE PSI VALUES FOR FIELD CASES (5-SPOT).----

$\mathrm{S} 1=(\mathrm{SW} 1 \mathrm{I}-\mathrm{SWR}) /(1.0-\mathrm{SWR}-\mathrm{SOR})$

$\mathrm{SI}=(\mathrm{SWII}-\mathrm{SWR}) /(1.0-\mathrm{SWR}-\mathrm{SOR})$

AKRWI =AKRWO*S1**ANW

$A K R W I=A K R W O * S I * * A N W$

AKROI $=$ AKROO* $(1.0-S I) * *$ ANO

$A K R O I=A K R O O^{*}(1.0-S I) * A N O$

IF( AKRO1 .LT. 0.00001) THEN

$A M I=1.0$

ELSE

$A M I=A K R W O * A M U O /\left(A K R O I^{*} A M U W\right)$

ENDIF

IF( AKROI .LT. 0.00001) THEN

$A M I=1.0$

ELSE

$A M I=A K R W O * A M U O /\left(A K R O I^{*} A M U W\right)$ 
ENDIF

PSI $=($ ALOG $(R E / R P M)+1.0 / A M I * A L O G(R E / R O)) / A L O G(R P M / R O)$

PSII $=\left(A L O G(R E / R P M)+1.0 / A M I^{*} A L O G(R E / R O)\right) / A L O G(R P M / R O)$

RETURN

END

$\mathrm{C}$

SUBROUTINE CALCI(RI,RRI,SWAVGI,DFWI)

C THIS SUBROUTINE CALCULATES THE CONSTANTS NECESSARY FOR

C EVALUATING THE LEFT-HAND-SIDE OF THE THEORETICAL EQUATION

C (LAYER I).

$\mathrm{C}$

COMMON/RK/ SOR,SWR,AKRWO,AKROO,PHI1,PHI,AKI,AKI,RDO,RDI,

\$ RDPM,RE,RO,RPI,RPM,ANO,ANW

COMMON/FLUD/ FR,AMUW,AMUO,AMUP,AMO,AMOP,SWIPF,SWIPF,SW1F,

$\$ \quad$ SWIF,SWIK,SWIK,SW1I,SWII,SWIINF,SWIINF,SWCT

COMMON/CONST/ NI,NO,SHK 11,SHK 12,SHKI11,SHKI12,SHK21,

$\$ \quad$ SHK 22,SHKI21,SHK122,AINF1,AINF2,AINFI1,

$\$ \quad$ AINFI2,SWCT1,SWCT2,IFLAG

$F R=A M U P / A M U W$

$A M O=A K R W O * A M U O /\left(A M U W^{*} A K R O O\right)$

$A M O P=A K R W O * A M U O /(A M U P * A K R O O)$

IF(ABS(FR-1.0).GT.0.0001) THEN

CALL SHOCK 1(AMOP,SHKI11,SHKI12,SWIPF)

CALL SHOCK2,(AMO,AMOP,SWIPF,SHKI21,SHKI22,SWIK)

CALL SWLMT(AMO,AMOP,SWIPF,SWCT1,SWCT2,SWCT)

IF(SWII.GT.SWCT) IHEN

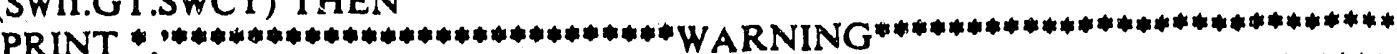

$* * * * * *$

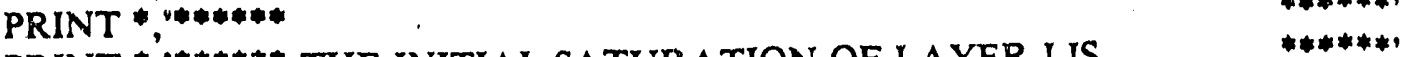

PRINT *;***** THE INITIAL SATURATION OF LA YER I IS

PRINT ***** GREATER THAN THE CRITICAL SATURATION,

PRINT ****** AN APPROXIMATE ESTIMATE OF THE DEGREE

PRINT , ****** OF PENETRATION IS ATTEMPTED BY ******,

PRINT *,****** RESETTING SWII TO (1-SOR).

PRINT *,

PRINT $*, * * * * * * * * * * * * * * * * * * * * *$ W ARNING

SWII $=1.0-S O R$

ENDIF

ENDIF

IF(A.BS(SWII-(1.0-SOR)).LT.0.0001) THEN

RRI $=1.0$

$\mathrm{RI}=1.0$

ELSE

IF(ABS(FR-1.0).LT.0.0001) THEN

C -.--CALCULATE THE FRONTAL SATURATIONS, SWAVG AND THE VELOCITY

C RATIO OF OIL BANK AND GELANT FRONT WHEN FR=1.0..---

$\mathrm{RI}=0.0$

CALL INF(AMO,AINFI1,AINFI2,SWIINF)

IF( SWII .LT. SWIINF) THEN

CALL SHOCK(AMO,SWII,SHK121,SHKI22,SWIF)

CALL FW(AMO,SWIF,FWIF)

CALL DFDS(AMO,SWIF,DFWI) 


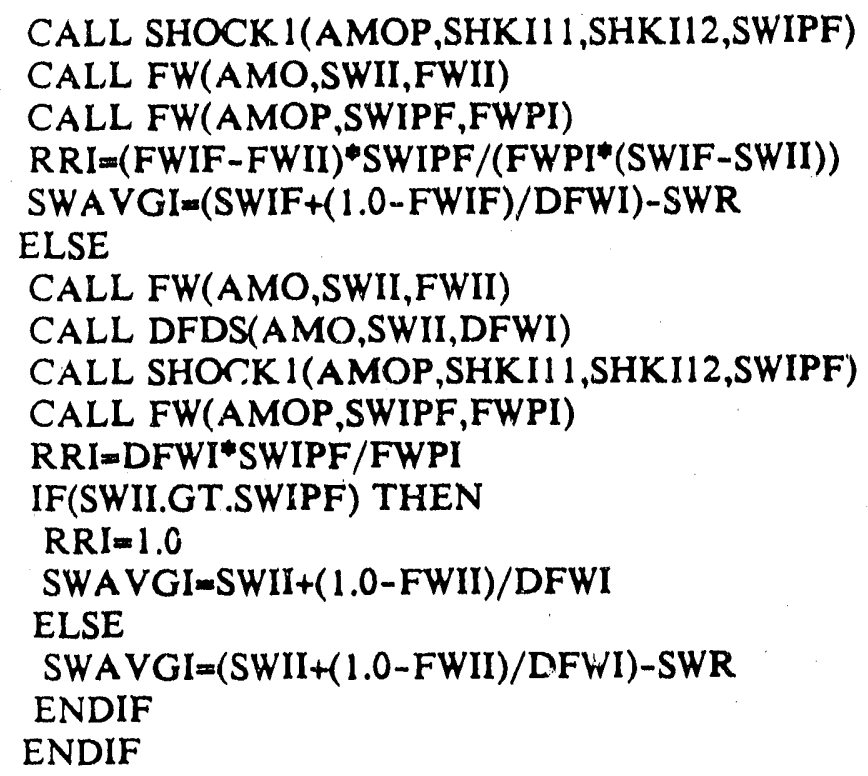


IF:(SWII.GT.SWCT) THEN

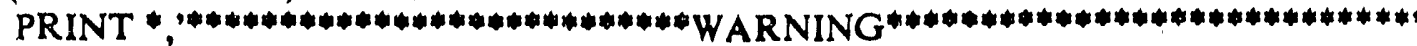

PRINT * ****** ******1

PRINT ****** THE INITIAL SATURATION OF LAYER IIS *****

PRINT ***** GREATER THAN THE CRITICAL SATURATION, ******

PRINT ****** AN APPROXIMATE ESTIMATE OF THE DEGREE ******

PRINT ******* OF PENETRATION IS ATTEMPTED BY

PRINT ****** RESETTING SWII TO (1-SOR). ******1

PRINT ******* RESETTING SWII TO (I,

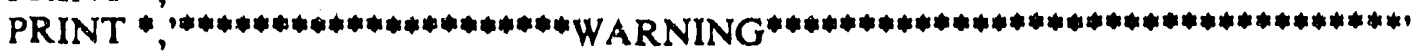

$S W 1 I=1.0-S O R$

ENDIF

ENDIF

IF(ABS(SWII-(1.0-SOR)).LT.0.0001) THEN

$\mathrm{RR} I=1.0$

$\mathrm{R} I=1.0$

IF( IFLAG.EQ.1) THEN

C ---CALCULATE THE FRONTAL SATURATIONS AND THE VELOCITY RATIO OF

C OIL BANK AND GELANT FRONT WHEN THE LAYER IS WATERED-OUT

C (COREFLOOD CASES).-.--

IF(ABS(FR-1.0).LT.0.0001) THEN

$R D I=1.0$

RETURN

ENDIF

RDI $=1.0 / R I$

ENDIF

ELSE

IF(ABS(FR-1.0).LT.0.0001) THEN

C -..-CALCULATE THE FRONTAL SATURATIONS, SWAVG AND THE VELOCITY

C RATIO OF OIL BANK AND GELANT FRONT WHEN FR=1.0....-

$R I=0.0$

IF( IFLAG.EQ.1) THEN

C ----SET RDI $=1.0$ FOR COREFLOOD CASES WHEN FR $=1.0 . \ldots$

$\mathrm{RDI}=1.0$

ENDIF

CALL INF(AMO,AINF1,AINF2,SWIINF)

IF( SWII .LT. SWIINF) THEN

CALL SHOCK(. MO,SW1I,SHK21,SHK22,SW1F)

CALL FW(AM ,JWIF,FWIF)

CALL DFDS(AMO,SWIF,DFWI)

CALL SHOCKI(AMOP,SHK 11,SHK 12,SW1PF)

CALL FW(AMO,SWII,FWII)

CALL FW(AMOP,SW1PF,FWP1)

$R R 1=(F W I F-F W 11)^{*} S W \perp P F /(F W P I *(S W I F-S W 1 I))$

SWA VG $1=(S W 1 F+(1.0-F W 1 F) / D F W 1)-S W R$

ELSE

CALL FW(AMO,SWII,FWII)

CALL DFDS(AMO,SW 11,DFW1)

CALL FWKRW(AMO,SW1I,FKRI)

CALL SHOCK 1(AMOP,SHK11,SHK 12,SW1PF)

CALL FW(AMCP,SWIPF,FWPI)

$R R I=D F W I * S W I P F / F W P I$ 
IF(SWII.GT.SWIPF) THEN

$R R I=1.0$

SWAVG I =SW 1 I $+(1.0-F W 11) / D F W 1$

ELSE

SWAVGI $=($ SW $1 I+(1.0-F W 1 I) / D F W 1)-S W R$

ENDIF

ENDIF

ELSE

C ---CALCULATE THE FRONTAL SATURATIONS, SWAVG AND THE VELOCITY

C RATIO OF OIL BANK AND GELANT FRONT WHEN FR $>1.0, \ldots$

RR $1=1.0$

CALL SHOCK 1(AMOP,SHK11,SHK 12,SW1PF)

CALL SHOCK2(AMO,AMOP,SWIPF,SHK21,SHK22,SW1K)

CALL FW(AMO,SWIK,FWIK)

CALL FW(AMO,SWII,FWII)

CALL FW(AMOP,SWIPF,FWPI)

$R I=(F W 1 K-F W 1 I) * S W I P F /(F W P 1 *(S W \mid K-S W I I))$

IF( IFLAG.EQ.1) THEN

RDI $=1.0 / R 1$

ENDIF

CALL DFDS(AMOP,SWIPF,DFWI)

SWAVG $1=S W 1 P F+(1.0-F W P I) / D F W I$

ENDIF

ENDIF

R.ETURN

END

$\mathrm{C}$

SUBROUTINE DFDS(XMO,XSW,DFW)

C THIS SL OUTINE CALCULATES THE VALUE OF FIRST DERIVATIVE OF A

C WATER FRACTIONAL FLOW FUNCTION.

C

COMMON/RK/ SOR,SWR,AKRWO,AKROO,PHII,PHII,AKI,AKI,RDO,RDI,

S RDPM,RE,RO,RPI,RPM,ANO,ANW.

$S=(X S W-S W R) /(1.0-S W R-S O R)$

$D F W=\left(A N W^{*} X M O * S^{* *}(A N W-1) *(1.0-S) * * A N D+A N O * X M O * S^{* *} A N W^{*}\right.$

$\$(1.0-S) *($ ANO- 1$)) /\left(\left(\mathrm{XMO}^{*} \mathrm{~S}^{* *} \mathrm{ANW}+(1.0-\mathrm{S}) *\right.\right.$ ANO $) * 2.0 *$

$\$ \quad(1.0-S W R-S O R))$

RETURN

END

SUBROUTINE D2FDS2(XMO,XSW,DDFW)

C

C THIS SUBROUTINE CALCULATES THE VALUE OF SECOND DERIVATIVE OF A

C WATER FRACTIONAL FLOW FUNCTION.

C

COMMON/RK/ SOR,SWR,AKRWO,AKROO,PHI,PHI, AK1,AKI,RDO,RDI,

$\$ \quad$ RDPM,RE,RO,RPI,RPM,ANO,ANW

$\mathrm{S}=(\mathrm{XSW}-\mathrm{SWR}) /(1.0-\mathrm{SWR}-\mathrm{SOR})$

DDFW $=\left(\left(X M 0^{*} \mathrm{~S}^{* *} \mathrm{ANW}+(1.0-\mathrm{S})^{* *} \text { ANO }\right)^{*}\left(\mathrm{ANW} *(\mathrm{ANW}-1) * \mathrm{XMO}^{*}\right.\right.$

$\$ S^{* *}(A N W-2) *(1 . C-S) *$ ANO-ANO*(ANO-1)*XMO* S $^{* *}$ ANW*

$\$ \quad(1.0-S) * *(A N O-2))-2^{*}\left(\right.$ ANW XMO* ${ }^{* *}(\text { ANW-1 })^{*}(1.0-S)^{* *}$ ANO+ 


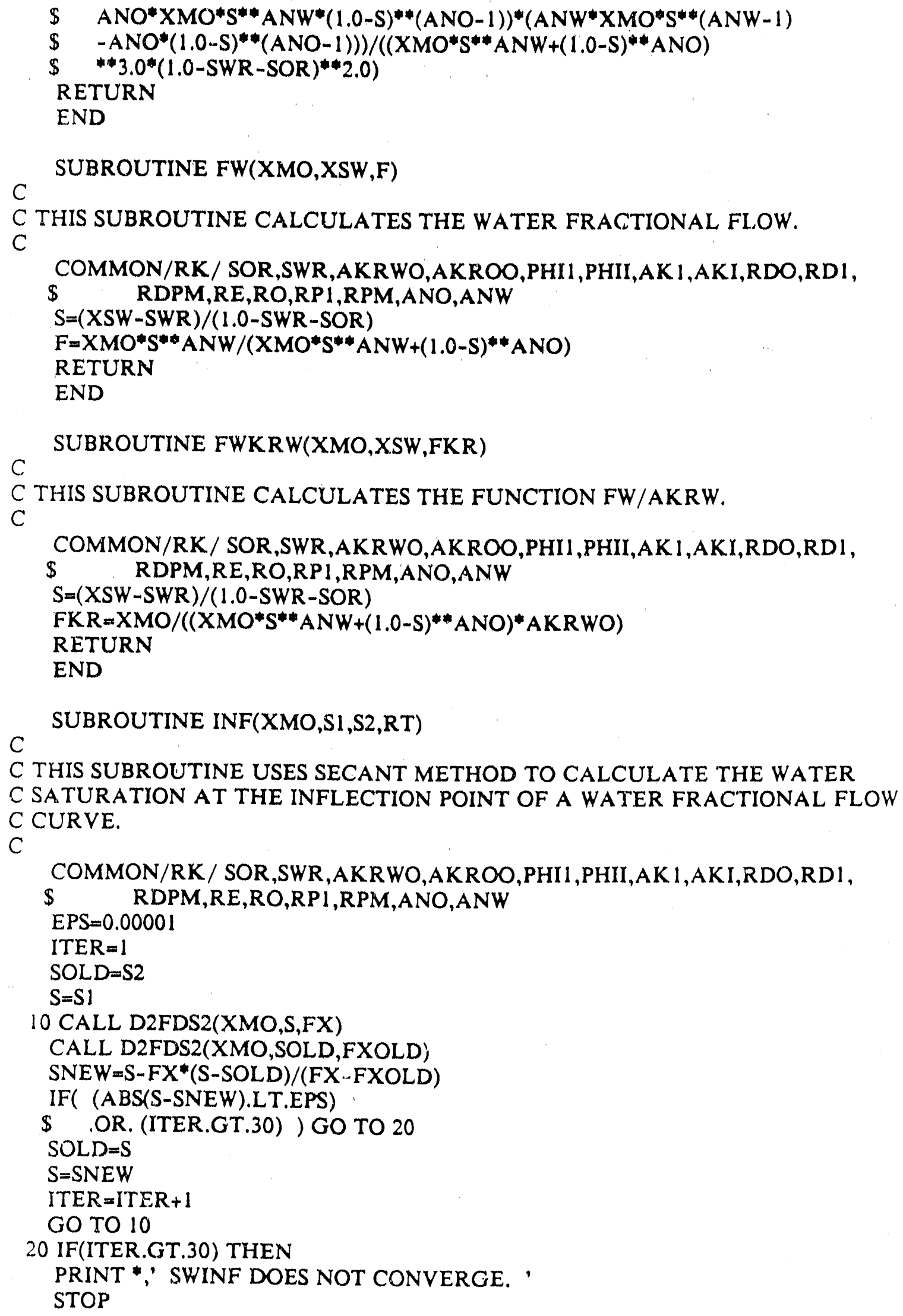


ELSE

RT $=$ SNEW

ENDIF

RETURN

END

SUBROUTINE INPUTT

C

C THIS SUBROUTINE READS THE NECESSARY INFORMATION FOR SUBSEQUENT

C CALCULATIONS.

$\mathrm{C}$

COMMON/RK/ SOR,SWR,AKRWO,AKROO,PHI1,PHII,AK1,AKI,RDO,RDI,

\$ RDPM,RE,RO,RPI,RPM,ANO,ANW

COMMON/FLUD/FR,AMUW,AMUO,AMUP,AMO,AMOP,SW 1PF,SWIPF,SWIF,

\$ SWIF,SWIK,SWIK,SWII,SWII,SWIINF,SWIINF,SWCT

COMMON/CONST/ NI,NO,SHK 11 ,SHK 12,SHK 111 ,SHKI12,SHK 21 ,

$\$$ SHK 22,SHK 12 1 ,SHK122,AINF1, AINF2,AINFI1,

$\$ \quad$ AINFI2,SWCT1,SWCT2,IFLAG

PRINT *, 'INPUT SOR,SWR'

READ(*,*) SOR,SWR

PRINT *, 'INPUT SW 11,SWII'

READ $\left.{ }^{*}, *\right)$ SWII,SWII

PRINT *, 'NPUT KRWO,KROO'

READ(*,") AKRWO,AKROO

PRINT ", 'INPUT ANW,ANO'

READ(*, ) ANW,ANO

PRINT *, 'INPUT PHII,PHII'

READ(*,*) PHII,PHII

PRINT *, 'INPUT KI, KI'

READ(*,*) AKI,AKI

PRINT *, 'INPUT MUW,MUO,MUP'

READ(*) AMUW,AMUO,AMUP

PRINT * 'INPUT IFLAG; IFLAG $=1--$ COREFLOOD, IFLAG=2--5-SPOT

READ(*, ) IFLAG

IF(IFLAG.EQ.2) THEN

PRINT *, 'INPUT RO,RPI, RPM,RE'

READ(*,*) RO,RPI,RPM,RE

ELSE

PRINT *, 'INPUT RO,RE'

READ(*, * RO,RE

C - . - LET RPM $=$ RE FOR. COREFLOOD CASES...--

$R P M=R E$

ENDIF

PRINT *, 'INPUT SHK 11,SHK 12,SHKI11,SHKI12'

READ **) SHK 11, SHK 12 ,SHK I11,SHK I12

PRINT *, 'INPUT SHK21,SHK22,SHKI21,SHKI22'

READ(*,) SHK 21,SHK 22,SHKI21,SHK 122

PRINT *, 'INPUT AINFI, AINF2, AINFII, AINFI2'

READ *, ) AINF1, AINF2,AINFI1,AINFI2

PRINT ", 'INPUT SWCT1,SWCT2'

READ***) SWCT1,SWCT2

PRINT *', 'INPUT NO. OF INTERVALS' 


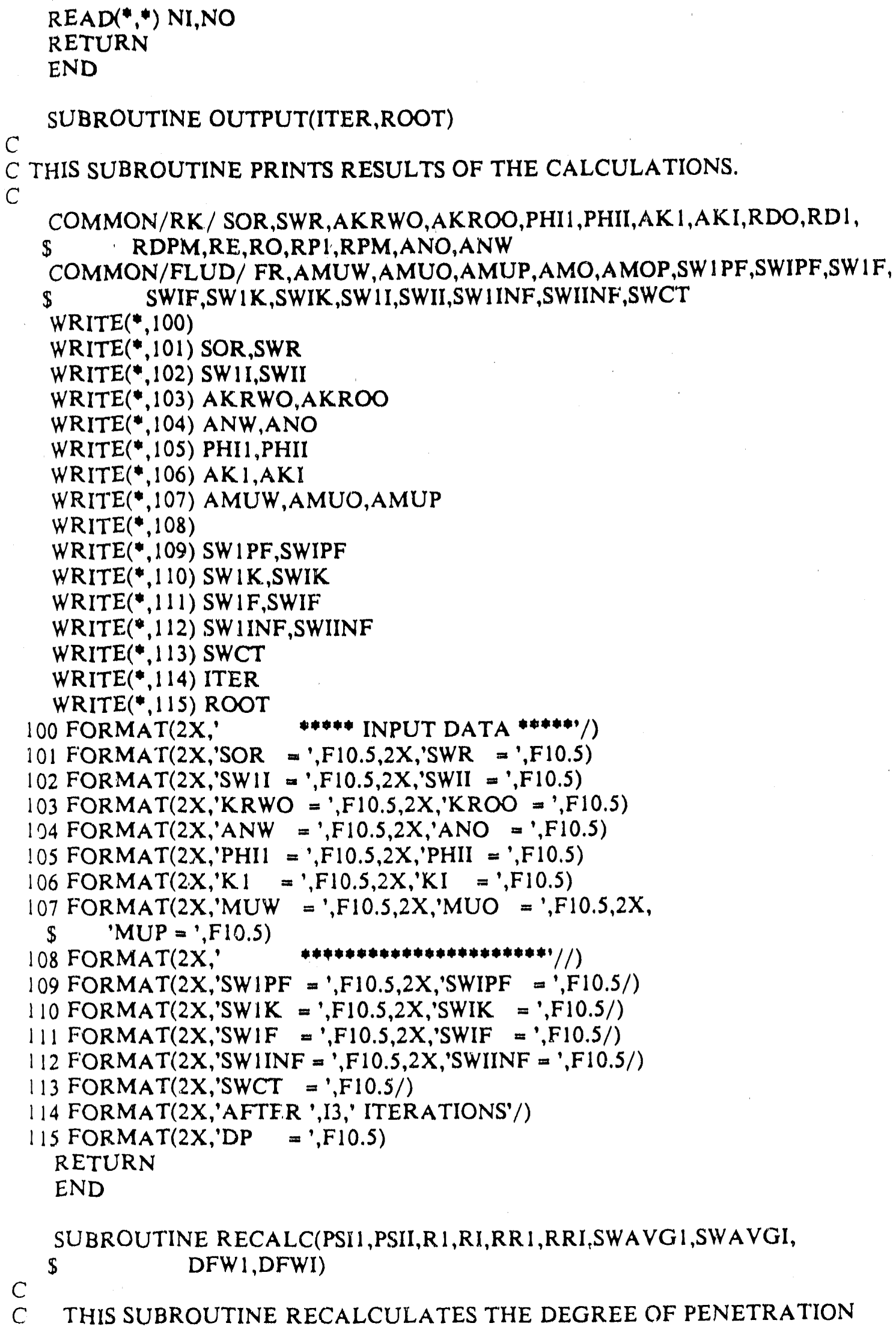


C WHEN THE GELANT FRONT OR THE OIL BANK IN LAYER I PROPAGATES

C BEYOND THE OUTLET OF THE CORE (COREFLOOD CASES) OR RPM

C (FIELD CASES) BY REPOSITIONING THE GELANT FRONT OR THE OIL

C BANK IN LAYER I AT THE OUTLET OF THE CORE OR RPM AND SOLVING

C. FOR THE POSITION OF THE GELANT FRONT IN LAYER 1.

C

COMMON/RK/ SOR,SWR,AKRWO,AKROO,PHII,PHII,AKI,AKI,RDO,RDI, S RDPM,RE,RO,RPI,RPM,ANO,ANW

COMMON/FLUD/ FR,AMUW,AMUO,AMUP,AMO,AMOP,SWIPF,SWIPF,SW IF, $\$ \quad$ SWIF,SWIK,SWIK,SWII,SWII,SWIINF,SWIINF,SWCT

COMMON/CONST/ NI,NO,SHK 11,SHK12,SHKI11,SHKI12,SHK21,

$\$ \quad$ SHK22,SHK121,SHKI22,AINF1,AINF2,AINFI1,

$\$ \quad$ AINFI2,SWCT1,SWCT2,IFLAG

C -.--REPOSITION THE GELANT FRONT OR THE OIL BANK IN LAYER I----

IF(IFLAG.EQ.1) THEN

IF(ABS(FR-1.0).LT.0.0001) THEN

$\mathrm{RDI}=1.0$

ELSE

$\mathrm{RDI}=1.0 / \mathrm{RI}$

ENDIF

ELSE

IF(ABS(FR-1.0).LT.0.0001) THEN

RDI=RDPM

ELSE

$\mathrm{RDI}=\mathrm{RDPM} / \mathrm{RI}$

ENDIF

ENDIF

C ---CALCULATE THE LEFT-HAND-SIDE OF THE THEORETICAL

C EQUAITON (LA YER I)----

CALL ALHSF(RDI,RI,PSII,SWAVGI,DFWI,ALHS)

C -.--USE SECANT METHOD TO SOLVE FOR THE DEGREE OF PENETRATION

C OF GELANTS INTO LAYER 1--.-

EPS=1.0E-07

ITER $=1$

$X=0.0001$

$X O L D=0.5$

C ----EVALUATE THE RIGHT-HAND-SIDE OF THE THEORETICAL

C EQUATION (LAYER 1)----

$10 \mathrm{RDI}=\mathrm{X}$

CALL RHSF(RI,PSI,,SWAVGI,DFW I,RHS)

$F X=A L H S-R H S$

$\mathrm{RDI}=\mathrm{XOLD}$

CALL RHSF(R1,PSI1,SWAVG1,DFW1,RHSO)

FXOLD $=A L H S-R H S O$

$X N E W=X-F X *(X-X O L D) /(F X-F X O L D)$

IF( (ABS(X-XNEW) .LT. EPS)

$\$$.OR. (ITER.GT.30) ) GO TO 20

$X O L D=X$

$\mathrm{X}=\mathrm{XNEW}$

ITER $=$ ITER+1

GO TO 10

20 IF(ITER.GT.30) THEN 


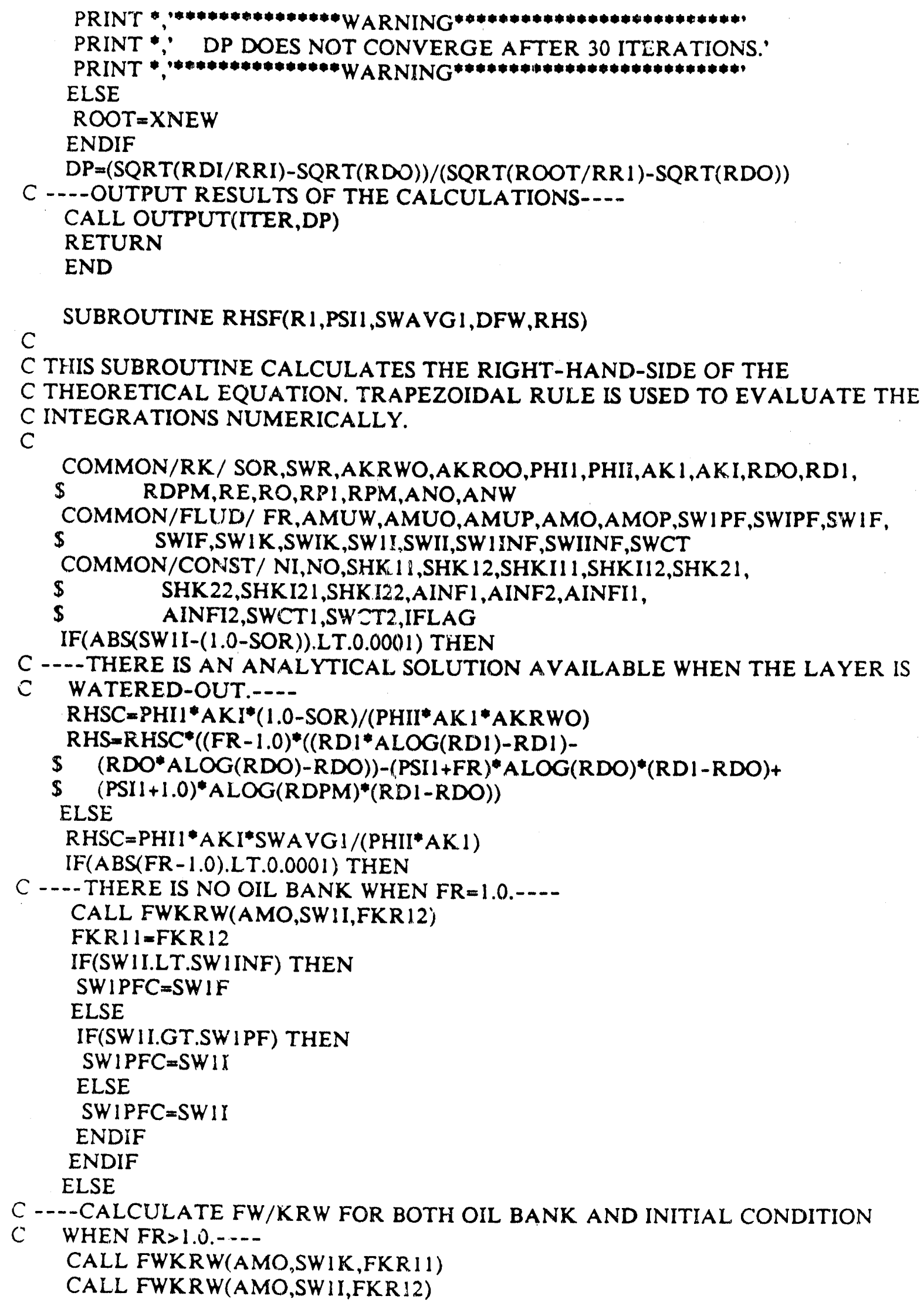


SWIPFC $=$ SWIPF

ENDIF

C----USE TRAPEZOIDAL RULE TO EVALUATE THE INTEGRATIONS

C NUMERICALLY.-.--

$\mathrm{H}=(\mathrm{RD} 1-\mathrm{RDO}) / \mathrm{NO}$

SUM $=0.0$

DO $100 \mathrm{I}=1, \mathrm{NO}-1$

$T=R D O+I^{*} H$

$T D=T / D F W$

CALL TRAPEZ(RDO,T,SWIPFC,NI,AMOP,TD,AR1)

IF(R1.LT.0.0001) THEN

$R \backslash T=0.0$

ELSE

$R I T=A L O S(R I * T)$

ENDIF

$R H S=(A R 1 * F R+F K R 11 *(R I T-A L O G(T))+F K R 12 *(A L O G(R D P M)$

$\left.\left.\$ \quad-R I T+P S I I^{*}(A L O G(R D P M)-A L O G(R D O))\right)\right) * R H S C$

$S U M=S U M+R H S$

100 CONTINUE

TD=RDI/DFW

CALL TRAPEZ(RDO,RDI,SWIPFC,NI,AMOP,TD,ARI)

IF(RI.LT.0.0001) THEN

$R \mid R D I=0.0$

$R I R D O=0.0$

ELSE

$R \mid R D I=A L O G(R I * R D I)$

$R I R D O=A L O G(R I * R D O)$

ENDIF

RHSB=(ARI*FR+FKR11*(RIRDI-ALOG(RDI))+FKR 12*(ALOG(RDPM)

$\left.\left.\$ \quad-R \backslash R D I+P S I I^{*}(A L O G(R D P M)-A L O G(R D O))\right)\right)^{*} R H S C$

RHSA $=\left(F K R 11^{*}(R 1 R D O-A L O G(R D O))+F K R 12 *(A L O G(R D P M)\right.$

$\left.\left.\$ \quad-R I R D O+P S I I^{*}(A L O G(R D P M)-A L O G(R D O))\right)\right)^{*} R H S C$

$\mathrm{RHS}=(\mathrm{H} / 2.0)^{*}(\mathrm{RHSB}+2.0 * \mathrm{SUM}+\mathrm{RHSA})$

ENDIF

RETURN

END

$\mathrm{C}$

SUBROUTINE SECANT(XMO,TD,X,S1,S2,RT)

C THIS SUBROUTINE USES SECANT METHOD TO SOLVE FOR THE WATER

C SATURATION PROFIL.E.

$\mathrm{C}$

COMMON/RK/ SOR,SWR,AKRWO,AKROO,PHI1,PHII,AK1,AKI,RDO,RDI,

$\$ \quad$ RDPM, RE, RO, RPI, RPM, ANO,ANW

$E P S=0.0001$

ITER $=1$

SOLD $=$ S2

$\mathrm{S}=\mathrm{SI}$

10 CALL XDS(S,XMO,X,TD,FX)

CALL XDS(SOLD,XMO,X,TD,FXOLD)

SNEW $=S-F X *(S-S O L D) /(F X-F X O L D)$

IF( (ABS(S-SNEW).LT.EPS) 


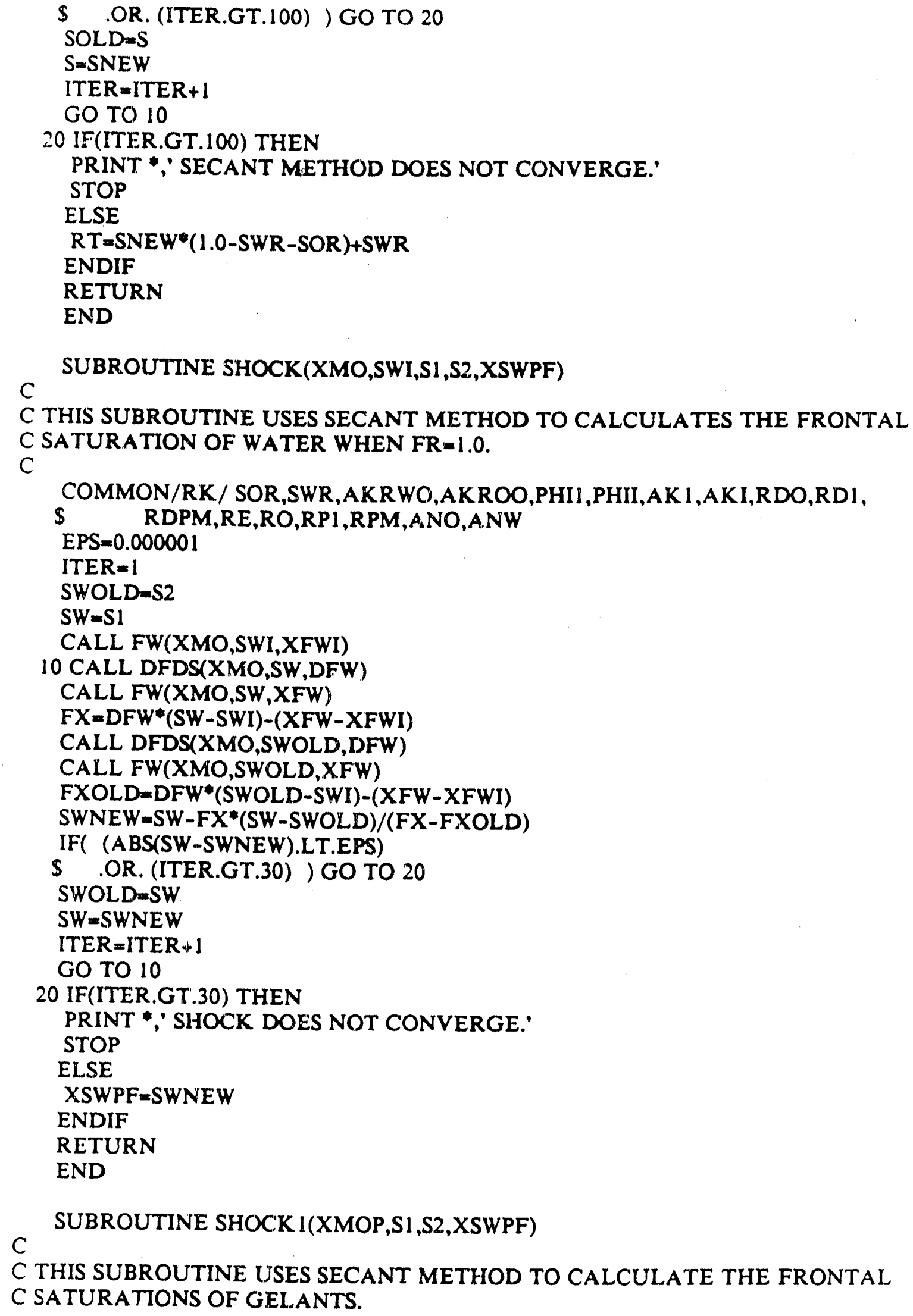


C

COMMON/RK/SOR,SWR,AKRWO,AKROO,PHI1,PHII,AKI,AKI,RDO,RDI, $\$ \quad$ RDPM,RE,RO,RPI,RPM,ANO,ANW

EPS $=0.000001$

ITER $=1$

SWOLD $=S 2$

$\mathrm{SW}=\mathrm{S} 1$

10 CALL DFDS(XMOP,SW,DFW)

CALL FW(XMOP,SW,XFW)

$F X=D F W * S W-X F W$

CALL DFDS(XMOP,SWOLD,DFW)

CALL FW(XMOP,SWOLD,XFW)

FXOLD $=D F W * S W O L D-X F W$

SWNEW-SW-FX*(SW-SWOLD)/(FX-FXOLD)

IF( (ABS(SW-SWNEW).LT.EPS)

$\$$.OR. (ITER.GT.30) ) GO TO 20

SWOLD $=$ SW

$S W=S W N E W$

ITER $=$ ITER+1

GO TO 10

20 IF(ITER.GT.30) THEN

PRINT *'SHOCK I DOES NOT CONVERGE'

STOP

ELSE

$X S W P F=S W N E W$

ENDIF

RETURN

END

$\mathrm{C}$

SUBROUTINE SHOCK2(XMO,XMOP,XSWPF,S1,S2,XSWK)

C THIS SUBROUTINE USES SECANT METHOD TO CALCULATE THE FRONTAL

C SATURATIONS OF OIL BANKS.

$\mathrm{C}$

COMMON/RK/ SOR,SWR,AKRWO,AKROO,PHI1,PHII,AKI,AKI,RDO,RDI,

$\$ \quad$ RDPM,RE,RO,RPI,RPM,ANO,ANW

CALL, FW(XMOP,XSWPF, FWP)

FWPS $=F W P / X S W P F$

$E P S=0.00001$

ITER $=1$

SWOLD $=S 2$

$\mathrm{SW}=\mathrm{SI}$

10 CALL FW(XMO,SW,XFW)

$\mathrm{FX}=\mathrm{XFW} / \mathrm{SW}-\mathrm{FWPS}$

CALL FW(XMO,SWOLD,XFW)

FXOLD $=X F W / S W O L D-F W P S$

SWNEW $=S W-F X *(S W-S W O L D) /(F X-F X O L D)$

IF( (ABS(SW-SWNEW).LT.EPS)

$\$$.OR. (ITER.GT.30) ) GO TO 20

$S W O L D=S W$

$S W=S W N E W$

ITER $=$ ITER +1 


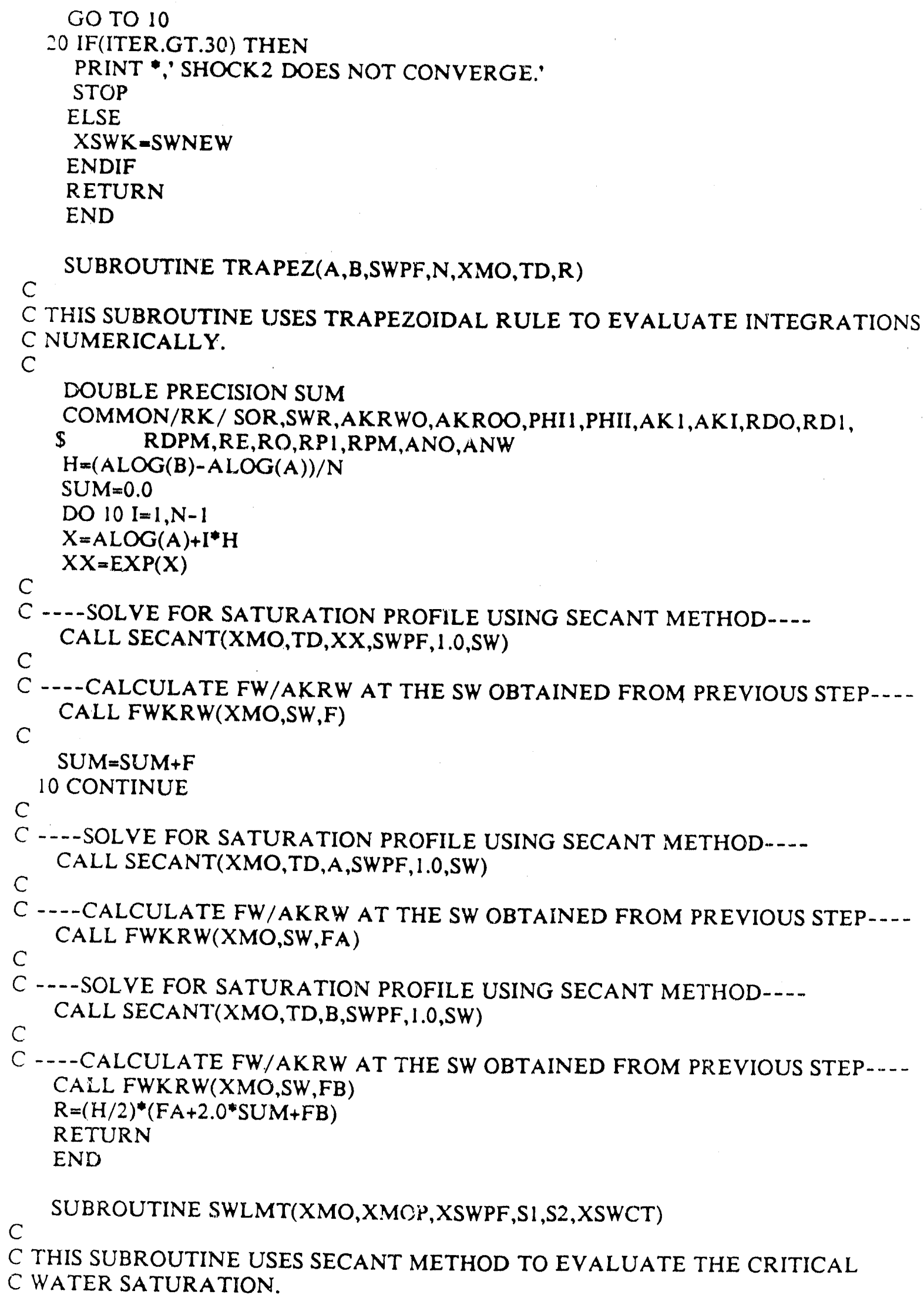


C

COMMON/RK/ SOR,SWR,AKRWO,AKROO,PHII,PHII,AKI,AKI,RDO,RDI,

$\$ \quad$ RDPM,RE,RO,RPI,RPM,ANO,ANW

$E P S=0.00001$

ITER $=1$

SWOLDES2

$S W=S 1$

CALL FW(XMOP,XSWPF,XFWP)

10 CALL FW(XMO,SW,XFW)

$F X=X F W / S W-X F W P / X S W P F$

CALL FW(XMO,SWOLD,XFW)

$F X O L D=X F W / S W O L D-X F W P / X S W P F$

$S W N E W=S W-F X *(S W-S W O L D) /(F X-F X O L D)$

IF( (ABS(SW-SWNEW).LT.EPS)

$\$$.OR. (ITER.GT.30) ) GO TO 20

SWOLD=SW

$S W=S W N E W$

ITER $=$ ITER +1

GO TO 10

20 IF(ITER.GT.30) THEN

PRINT *' SWCT DOES NOT CONVERGE.'

STOP

ELSE

XSWCT $=S W N E W$

ENDIF

RETURN

END

C

SUBROUTINE XDS(S,XMO,X,TD,FS)

C THIS SUBROUTINE CALCULATES THE FUNCTION FX=XD-DFW/DSW TD FOR

C SUBROUTINE SECANT.

$\mathrm{C}$

COMMON/RK/ SOR,SWR,AKRWO,AKROO,PHI1,PHII,AKI,AKI,RDO,RDI, $\$ \quad$ RDPM,RE,RO,RPI,RPM,ANO,ANW

$F S=\left(A N W * X M O * S^{* *}(A N W-1) *(1-S)^{* *} A N O+A N O * X M O * S^{* *} A N W *\right.$

$\$(1.0-S)^{* *}($ ANO-1 $\left.)\right)^{*} \mathrm{TD}-\mathrm{X}^{*}\left(\mathrm{XMO}^{*} \mathrm{~S}^{* *} \mathrm{ANW}+(1.0-\mathrm{S})^{* *} \mathrm{ANO}\right) * 2.0 *$

$\$$ (1.0-SWR-SOR)

RETURN

END 
APPENDIX G

FORTRAN CODING FOR THEORETICAL MODEL: LINEAR FLOW 
C

C PROGRAM INFORMATION:

C

C BASED UPON THE THEORETICAL MODEL DEVELOPED IN THIS PAPER, A C FORTRAN PROGRAM WAS DEVELOPED TO DETERMINE THE DEGREE OF

C PENETRATION OF GELANTS INTO OIL-PRODUCTIVE STRATA AS WELL AS

C INTO WATER-SOURCE ZONES. THE TECHNIQUES USED IN THIS PROGRAM

C TO CALCULATE FRUNTAL SATURATIONS, SATURATION PROFILES, AND

C AVERAGE SATURATIONS CAN BE FOUND IN REFS. 103, 104, AND 105.

C

C PROGRAM LIMITATIONS:

C IN ADDITION TO THE ASSUMPTIONS MADE IN DEVELOPING THE

C THEORETICAL MODEL, THE I.IMITATIONS OF THIS FORTRAN PROGRAM

C ARE LISTED BELOW:

C 1. THIS PROGRAM DEALS WITH LINEAR CASES ONLY.

C 2. IT IS ASSUMED IN THIS PROGRAM THAT, IN ANY LAYER, THE

C OIL BANK (FR>1) OR THE WATEK FRONT (FR=1) DOESN'T

C PROPAGATE BEYOND (A) THE OUTLET OF THE CORE (COREFLOOD

C CASES) OR (B) RPM (FIELD CASES).

C 3. IT IS ASSUMED IN THIS PROGRAM THAT THE FIELD PATTERN IS

C 5-SPOT.

$\mathrm{C}$

C

C

C

C

C

C

C

C

C

C

C

C

C

C

C

C

C

C

C

C

C AKROO =

C $A K R W=$

C AKRWO=

C ALI =

$\mathrm{C}$

C

C ALHS $\quad=$ LEFT-HAND-SIDE OF THE THEORETICAL EQUATION

C AMO $=$ WATER-OIL MOBILITY 


\begin{tabular}{|c|c|c|c|}
\hline $\mathrm{C}$ & AMOP & $=$ & GELANT-OIL MOBILITY \\
\hline $\mathrm{C}$ & AMUO & $=$ & OIL VISCOSITY, CP \\
\hline $\mathrm{C}$ & AMUP & $=$ & GELANT VISCOSITY, CP \\
\hline C & AMUW & $=$ & WATER VISCOSITY, CP \\
\hline $\mathrm{C}$ & ANO & $=$ & EXPONENT FOR OIL RELATIVE PERMEABILLITY EQUATION \\
\hline $\mathrm{C}$ & ANW & $=$ & EXPONENT FOR WATER RELATIVE PERMEABILITY EQUATION \\
\hline $\begin{array}{l}\mathrm{C} \\
\mathrm{C}\end{array}$ & AR1 & & $\begin{array}{l}\text { THE FIRST INTEGRATION TERM (GELANT FRONT) ON THE } \\
\text { RIGHT-HAND-SIDE OF THE THEORETICAL EQUATION } \\
\text { (LAYER 1) }\end{array}$ \\
\hline $\begin{array}{l}\text { C } \\
\text { C }\end{array}$ & DFW & $=$ & $\begin{array}{l}\text { FIRST DERIVATIVE OF THE WATER FRACTIONAL FLOW } \\
\text { FUNCTION (FW) }\end{array}$ \\
\hline $\begin{array}{l}\mathrm{C} \\
\mathrm{C}\end{array}$ & DDFW & $=$ & $\begin{array}{l}\text { SECOND DERIVATIVE OF THE WATER FRACTIONAL FLOW } \\
\text { FUNCTION (FW) }\end{array}$ \\
\hline $\begin{array}{l}\mathrm{C} \\
\mathrm{C}\end{array}$ & DP & $=$ & $\begin{array}{l}\text { DEGREE OF PENETRATION OF GELANTS (AS DEFINED IN } \\
\text { THE PAPER) }\end{array}$ \\
\hline $\mathrm{C}$ & EPS & $=$ & CONVERGENCE CRITERIA \\
\hline C & FKR 11 & $=$ & FW/AKRW AT SWIK (OIL BANK IN LAYER 1) \\
\hline C & FKR12 & $=$ & FW/AKRW AT SWII (INITIAL CONDITION IN LAYER 1) \\
\hline C & FKRII & 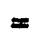 & FW/AKRW AT SWIK (OIL BANK IN LAYER I) \\
\hline C & FKRI2 & $=$ & FW / AKRW AT SWII (INITIAL CONDITION IN LAYER I) \\
\hline $\begin{array}{l}\mathrm{C} \\
\mathrm{C}\end{array}$ & FR & $=$ & $\begin{array}{l}\text { RESISTANCE FACTOR (BRINE MOBILITY DIVIDED BY } \\
\text { MOBILITY OF THE UELANT) }\end{array}$ \\
\hline C & FW & $=$ & WATER FRACTIONAL FLOW FUNCTION \\
\hline $\begin{array}{l}\mathrm{C} \\
\mathrm{C}\end{array}$ & FWli & $=$ & $\begin{array}{l}\text { WATER FRACTIONAL FLOW AT INITIAL WATER } \\
\text { SATURATION IN LAYER } 1\end{array}$ \\
\hline C & FWIK & $=$ & $\begin{array}{l}\text { WATER FRACTIONAL FLOW AT OIL BANK FRONT IN } \\
\text { LA YER } 1\end{array}$ \\
\hline C & FWII & $=$ & $\begin{array}{l}\text { WATER FRACTIONAL FLOW AT INITIAL WATER } \\
\text { SATURATION IN LAYER I }\end{array}$ \\
\hline C & FWIK & $=$ & $\begin{array}{l}\text { WATER FRACTIONAL FLOW AT OIL BANK FRONT IN } \\
\text { LAYER I }\end{array}$ \\
\hline $\mathrm{C}$ & FWP & $=$ & WATER FRACTIONAL FLOW AT GELANT FRONT \\
\hline C & ITER & $=$ & COUNTER OF THE NUMBER OF ITERATIONS \\
\hline $\begin{array}{l}\text { C } \\
C \\
C\end{array}$ & NI & $=$ & $\begin{array}{l}\text { NUMBER OF INTERVALS USED IN SUBROUTINE TRAPEZ TO } \\
\text { EVALUATE THE INSIDE INTEGRATION TERMS } \\
\text { NUMERICALLY }\end{array}$ \\
\hline $\begin{array}{l}\mathrm{C} \\
\mathrm{C}\end{array}$ & NO & $=$ & $\begin{array}{l}\text { NUMBER OF INTERVALS USED IN EVALUATING THE } \\
\text { OUTSIDE INTEGRATIONS }\end{array}$ \\
\hline C & PHII & $=$ & POROSITY IN LAYER 1 \\
\hline C & PHII & $=$ & POROSITY IN LA YER X \\
\hline $\begin{array}{l}C \\
C \\
C \\
C\end{array}$ & PSI & $=$ & $\begin{array}{l}\text { PRESSURE DROP BETWEEN RPM AND THE INJECTION WELL } \\
\text { DIVIDED BY THE PRESSURE DROP BETWEEN THE } \\
\text { PRODUCTION WELL AND RPM JUST PRIOR TO GELANT } \\
\text { INJECTION }\end{array}$ \\
\hline $\begin{array}{l}\mathrm{C} \\
\mathrm{C}\end{array}$ & RI & $=$ & $\begin{array}{l}\text { THE VELOCITY RATIO OF GELANT FRONT AND OIL BANK } \\
\text { IN LAYER } 1\end{array}$ \\
\hline C & RE & $=$ & DRAINAGE RADIUS, FT \\
\hline $\begin{array}{l}C \\
C\end{array}$ & RI & $=$ & $\begin{array}{l}\text { THE VELOCITY RATIO OF GELANT FRONT AND OIL BANK } \\
\text { IN LA YER I }\end{array}$ \\
\hline $\mathrm{C}$ & RO & $=$ & WELLBORE RADIUS, FT \\
\hline $\mathrm{C}$ & M & & $\begin{array}{l}\text { A REFERENCE DISTANCE FROM THE WELLBORE BEYOND } \\
\text { WHICH THE GEL TREATMENT HAS NO EFFECT ON FLUID }\end{array}$ \\
\hline
\end{tabular}




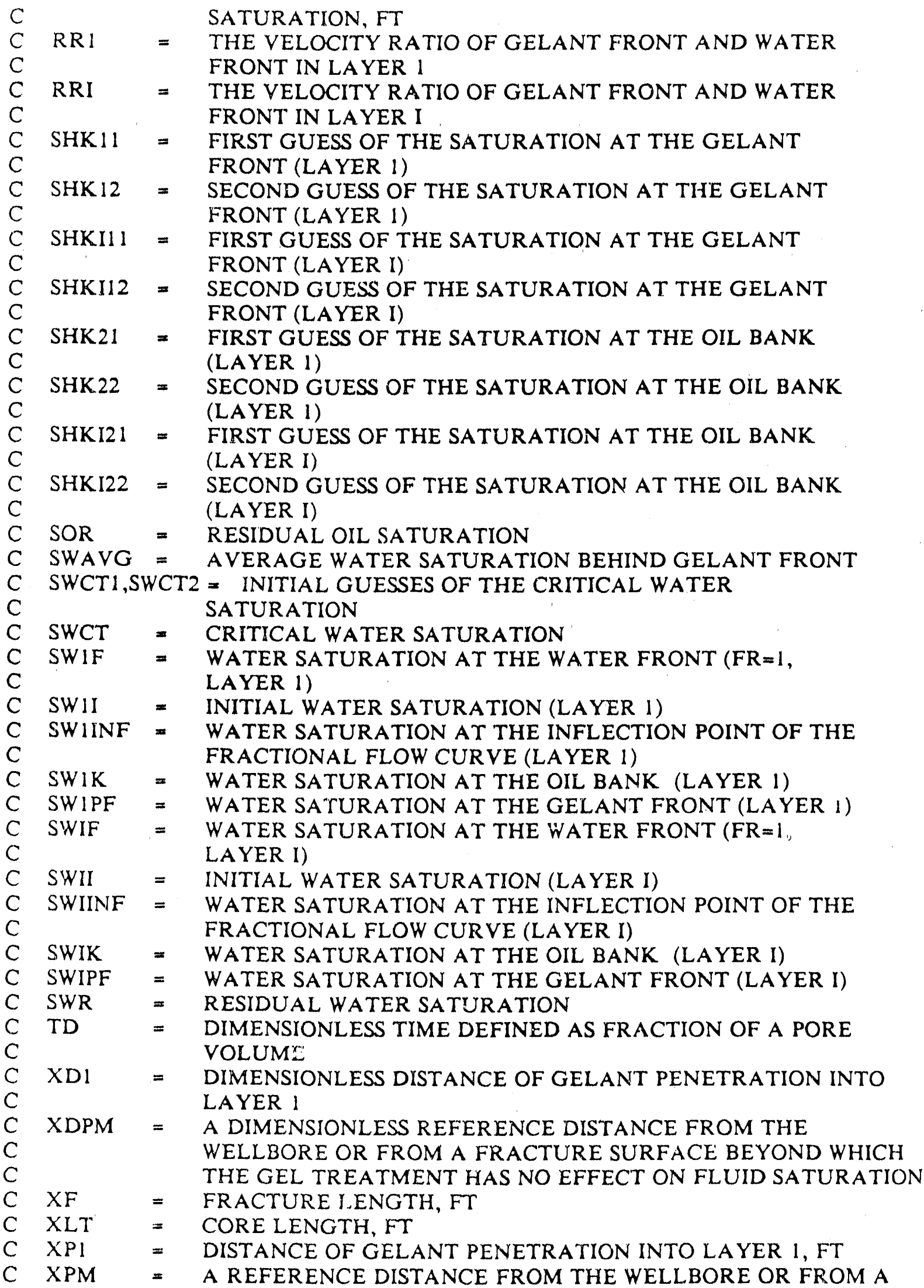




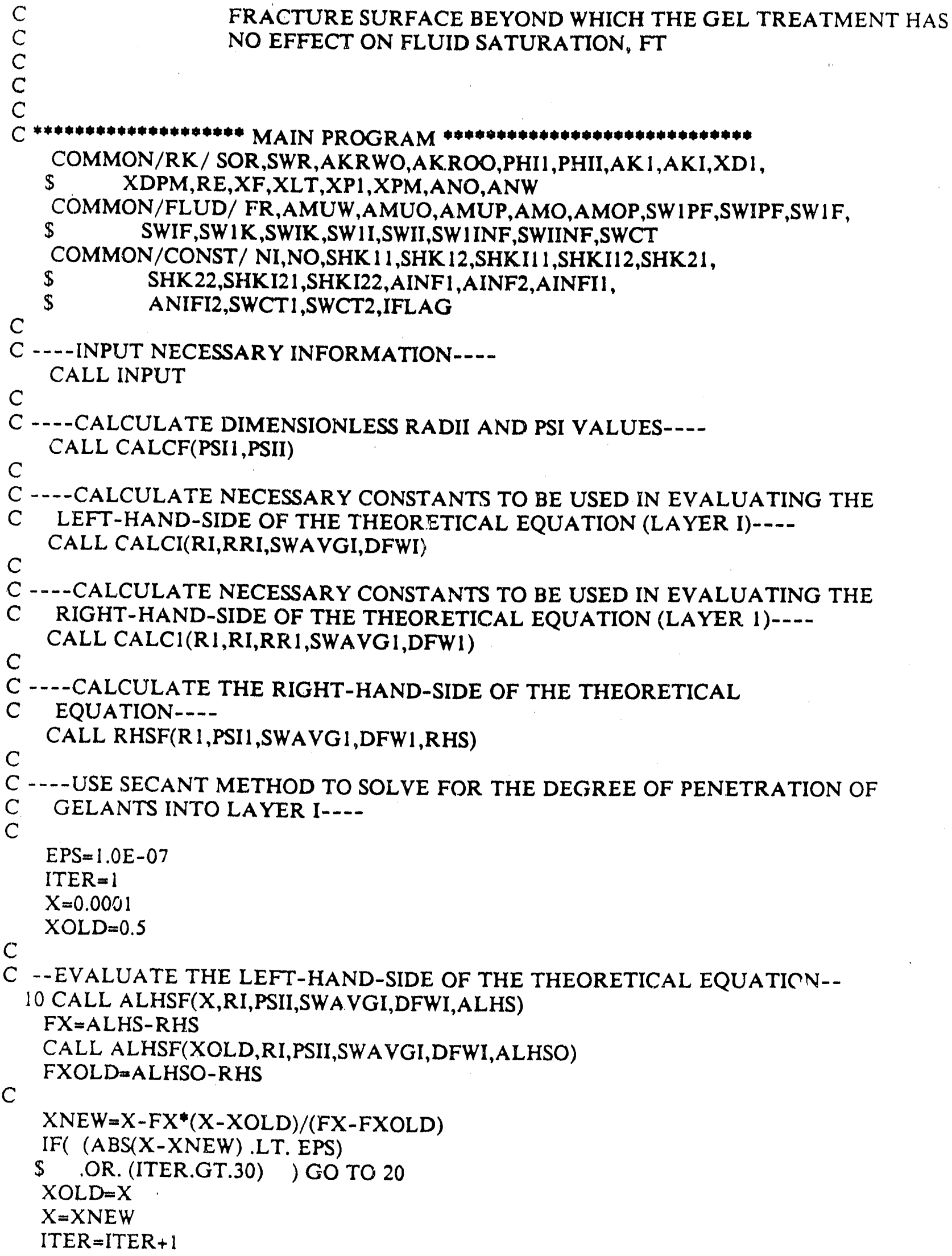




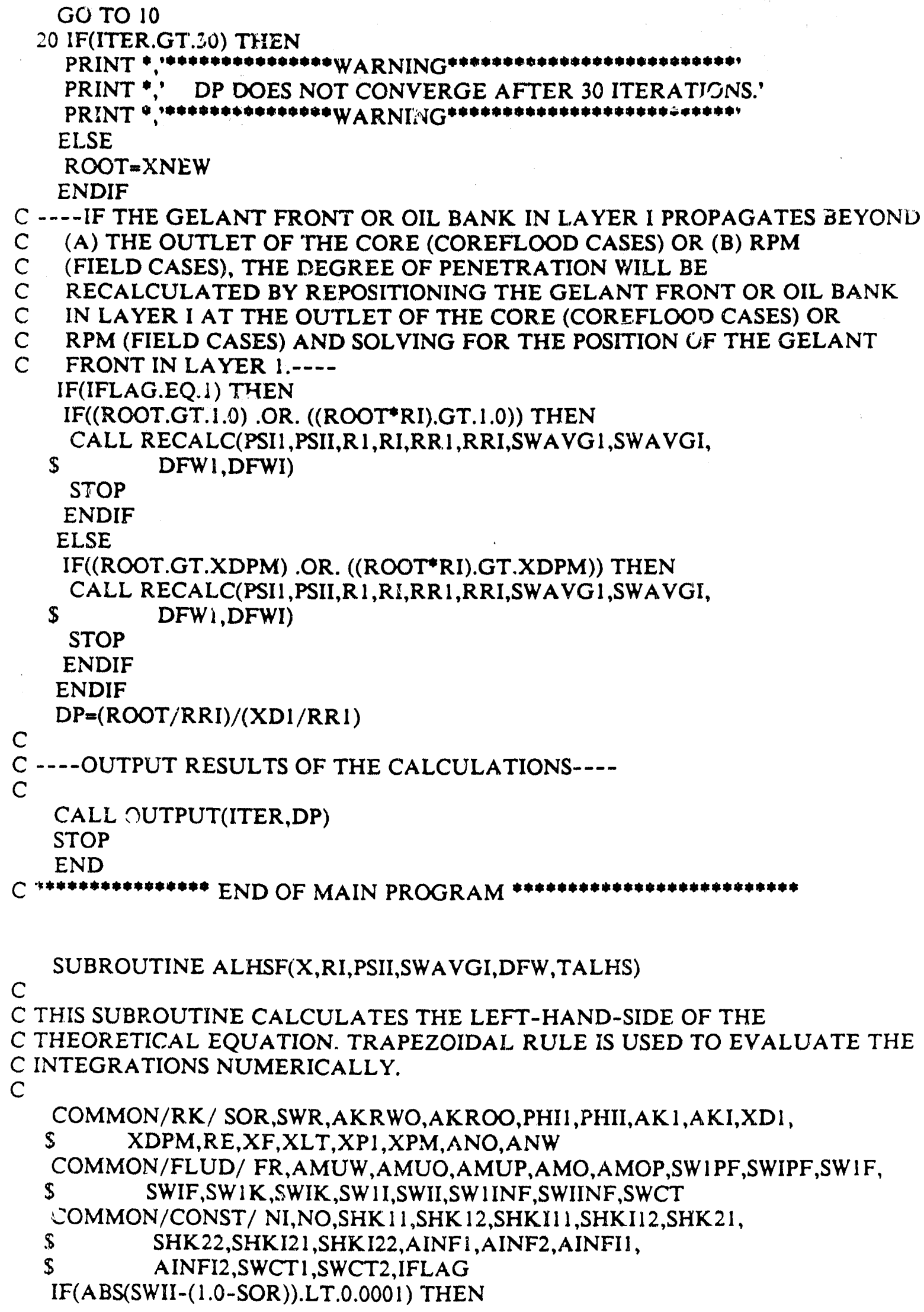


C ----THERE IS AN ANALYTICAL SOLUTION A VAILABLE WHEN THE LAYER IS

C WATERED-OUT.----

TALHS $=(1.0-S O R) / A K R W O *((F R-1.0) * 0.5 * X * 2.0+($ PSII +1.0$) *$

$\$ \quad X * X D P M)$

ELSE

IF(ABS(FR - 1.0).LT.0.0001) THEN

C -.--THERE IS NO OIL BANK WHEN FR=1.0...-

CALL FWKRW(AMO,SWII,FKRI2)

FKRII =FKRI2

IF(SWII.LT.SWIINF) THEN

SWIPFC=SWIF

ELSE

IF(SWII.GT.SWIPF) THEN

SWIPFC=SWII

ELSE

SWIPFC $=$ SWII

ENDIF

ENDIF

ELSE

C ---CALCULATE FW/KRW FOR BOTH CIL BANK AND INITIAL CONDITION

C WHEN FR>1.0...-

CALL FWKRW(AMO,SWIK,FKRII)

CALL FWKRW(AMO,SWII,FKRI2)

SWIPFC $=$ SWIPF

ENDIF

C----USE TRAPEZOIDAL RULE TO EVALUATE THE INTEGRATIONS

C NUMERICALLY.-..-

$\mathrm{H}=\mathrm{X} / \mathrm{NO}$

SUM $=0.0$

DO $200 \mathrm{I}=1, \mathrm{NO}-1$

$T=I^{*} H$

$T D=T / D F W$

CALL TRAPEZ(0.0,T,SWIPFC,NI,AMOP,TD,AL1)

IF(RI.LT.0.0001) THEN

RIT $=0.0$

ELSE

RIT $=R I^{\star} T$

ENDIF

ALHS $=(A L I * F R+F K R I 1 *(R I T-T)+F K R I 2 *(X D P M$

$\left.\$ \quad-R I T+P\left(S I I^{*} X D P M\right)\right) * S W A V G I$

$S U M=S U M+A L H S$

200 CONTINUE

$\mathrm{TD}=\mathrm{X} / \mathrm{DFW}$

CALL TRAPEZ(0.0,X,SWIPFC,NI,AMOP,TD,AL1)

IF(RI.LT.0.0001) THEN

RIX $=0.0$

ELSE

$\mathrm{RIX}=\mathrm{RI} I^{*} \mathrm{X}$

ENDIF

$A L H S B=(A L I * F R+F K R I *(R I X-X)+F K R I 2 *(X D P M$

$\left.\left.\$ \quad-R I X+P S I I^{*} X D P M\right)\right) * S W A V G I$

$A L H S A=(F K R I 2 *(X D P M$ 
$\$ \quad+$ PSII*XDPM))*SWAVGI

TALHS $=(\mathrm{H} / 2.0) *(\mathrm{ALHSB}+2.0 * \mathrm{SUM}+\mathrm{ALHSA})$

ENDIF

RETURN

END

$\mathrm{C}$

SUBROUTINE CALCF(PSII,PSII)

C THIS SUBROUTINE CALCULATES DIMENSIONLESS CONSTANTS AND PSI

C VALUES.

C

COMMON/RK/ SOR,SWR,AKRWO,AKROO,PHI,PHII,AKI,AKI,XDI, $\$ \quad X \Gamma P M, R E, X F, X L T, X P 1, X P M, A N O, A N W$

COMMON/FLUD/ FR,AMUW,AMUO,AMUP,AMO,AMOP,SWIPF,SWIPF,SWIF, $\$ \quad$ SWIF,SWIK,SWIK,SWII,SWII,SWIINF,SWIINF,SWCT

COMMON/CONST/ NI,NO,SHK 11,SHK 12,SHKI11,SHKI12,SHK21,

$\$ \quad$ SHK 22,SHK121,SHKI22,AINF1,AINF2,AINFI,

$\$ \quad$ ANIFI2,SWCT1,SWCT2,IFLAG

IF( IFLAG.EQ.2) THEN

C -.--CALCULATE DIMENSIONLESS CONSTANTS FOR FIELD CASES

C (5-SPOT)----

$D=R E * 3.14159 * * 0.5$

$X D I=X P 1 / D$

$X D P M=X P M / D$

ELSE

C ----SET PSI VALUES TO ZERO AND CALCULATE THE DIMENSIONLESS

C CONSTANTS FOR COREFLOOD CASES.----

$\mathrm{XDPM}=\mathrm{XPM} / \mathrm{XLT}$

$P S I 1=0.0$

PSII $=0.0$

RETURN

ENDIF

C -.--CALCULATE PSI VALUES FOR FIELD CASES (5-SPOT)...--

$\mathrm{S} I=(\mathrm{SW} 1 \mathrm{I}-\mathrm{SWR}) /(1.0-\mathrm{SWR}-\mathrm{SOR})$

$\mathrm{SI}=(\mathrm{SWII}-\mathrm{SWR}) /(1.0-\mathrm{SWR}-\mathrm{SOR})$

$A K R W I=A K R W O * S I * * A N W$

$A K R W I=A K R W O * S I * * A N W$

$A K R O 1=A K R O O *(1.0-S 1)^{* *}$ ANO

$A K R O I=A K R O O *(1.0-S I) * * A N O$

IF( AKROI .LT. 0.00001) THEN

$A M I=1.0$

ELSE

$A M 1=A K R W O * A M U O /\left(A K R O I^{*} A M U W\right)$

ENDIF

IF( AKROI .LT. 0.00001) THEN

$A M I=1.0$

ELSE

$A M I=A K R W O * A M U O /\left(A K R O I^{*} A M U W\right)$

ENDIF

$\mathrm{RO}=\mathrm{XF}$

$R P M=X F+X P M$

$\mathrm{PSI} I=\left(\mathrm{ALOG}(\mathrm{RE} / \mathrm{RPM})+1.0 / \mathrm{AM} 1^{*} \mathrm{ALOG}(\mathrm{RE} / \mathrm{RO})\right) / \mathrm{ALOG}(\mathrm{RPM} / \mathrm{RO})$ 
PSII $=($ ALOG(RE/RPM $)+1.0 /$ AMI $^{*}$ ALOG $\left.(R E / R O)\right) / A L O G(R P M / R O)$

RETURN

END

C

SUBROUTINE CALCI(RI,RRI,SWAVGI,DFWI)

C THIS SUBROUTINE CALCULATES THE CONSTANTS NECESSARY FOR

C EVALUATING THE LEFT-HAND-SIDE OF THE THEORETICAL EQUATION

C (LAYER I).

$\mathrm{C}$

COMMON/RK/ SOR,SWR,AKRWO,AKROO,PHII,PHII,AKI,AKI,XDI,

$\$ \quad X D P M, R E, X F, X L T, X P 1, X P M, A N O, A N W$

COMMON/FLUD/ FR,AMUW,AMUO,AMUP,AMO,AMOP,SWIPF,SWIPF,SWIF,

$\$ \quad$ SWIF,SW1K,SWIK,SWII,SWII,SWIINF,SWIINF,SWCT

COMMO ${ }^{r} /$ CONST/ NI,NO,SHK 11 ,SHK 12,SHKI11,SHKI12,SHK21,

$\$ \quad$ SHK 22,SHK121,SHKI22,AINF1,AINF2,AINFI,

$\$ \quad$ AINFI2,SWCT1,SWCT2,IFL.AG

$F R=A M U P / A M U W$

$A M O=A K R W O * A M U O /\left(A M U W^{*} A K R O O\right)$

$A M O P=A K R W O * A M U O /\left(A M U P^{*} A K R O O\right)$

IF(ABS(FR-1.0).GT.0.0001) THEN

CALL SHOCKI(AMOP,SHKI11,SHKI12,SWIPF)

CALL SHOCK2(AMO,AMOP,SWIPF,SHKI21,SHKI22,SWIK)

CALL SWLMT(AMO,AMOP,SWIPF,SWCT1,SWCT2,SWCT)

IF(SWII.GT.SWCT) THEN

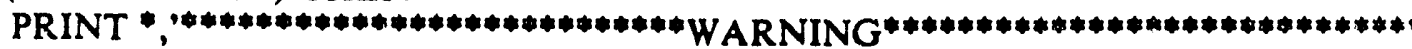

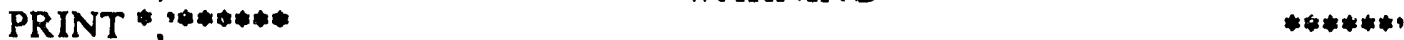

PRINT *;***** THE INITIAL SATURATION OF IAYER IIS

PRINT , ;***** GREATER THAN THE CRITICAL SATURATION, ******,

PRINT ****** AN APPROXIMATE ESTIMATE OF THE DEGREE ******1

PRINT ******* OF PENETRATION IS ATTEMPTED BY

PRINT ****** RESETTING SWII TO (1-SOR).

PRINT *

PRINT *,,$* * * * * * * * * * * * * * * * * * *$ WARNING

SWII $=1.0-S O R$

ENDIF

ENDIF

IF(ABS(SWII-(1.0-SOR)).LT.0.0001) THEN

RRI $=1.0$

$R I=1.0$

ELSE

IF(ABS(FR-1.0).LT.0.0001) THEN

C ---CALCULATE THE FRONTAL SATURATIONS, SWAVG AND THE VELOCITY

C RATIO OF OIL BANK AND GELANT FRONT WHEIN FR=1.0....-

$\mathrm{RI}=0.0$

CALL INF(AMO,AINFI1,AINFI2,SWIINF)

IF( SWII .LT. SWIINF) THEN

CALL SHOCK(AMO,SWII,SHKI21,SHKI22,SWIF)

CALL FW(AMO,SWIF,FWIF)

CALL DFDS(AMO,SWIF,DFWI)

CALL SHOCKI(AMOP,SHKI11,SHKI12,SWIPF)

CALL FW(AMO,SWII,FWII) 


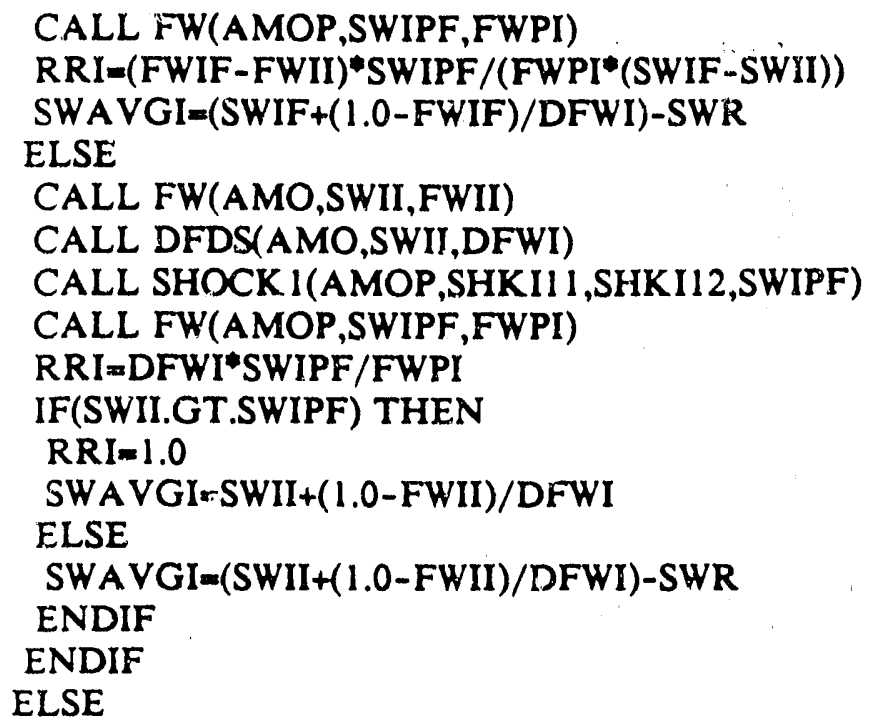

C ---CALCULATE. THE FRONTAL SATURATIONS, SWAVG AND THE VELOCITY

C RATIO OF OIL BANK AND GELANT FRONT WHEN FR>1.0.-.--

RRI $=1.0$

CALL SHOCK I(AMOP,SHKII1,SHKII2,SWIPF)

CALL SHOCK2(AMO,AMOP,SWIPE,SHKI21,SHK122,SWIK)

CALL FW(AMO,SWIK,FWIK)

CALL FW(AMO,SWII,FWII)

CALL FW(AMOP,SWIPF,FWPI)

RI=(FWIK-FWII) ${ }^{*}$ SWIPF/(FWPI*(SWIK-SWII))

CALL DFDS(AMOP,SWIPF,DFWI)

SWA VGI=SWIPF+(1.0-FWPI)/DFWI

ENDIF

ENDIF

RETURN

END

C

SUBROUTINE CALCI(RI,RI,RRI,SWAVGI,DFW1)

C THIS SUBROUTINE CALCULATES THE CONSTANTS NECESSARY FOR

C EVALUATING THE RIGHT-HAND-SIDE OF THE THEORETICAL EQUATION

C (LAYER 1).

$\mathrm{C}$

COMMON/RK/ SOR,SWR,AKRWO,AKROO,PHII,PHII,AK1,AKI,XDI, $\$ \quad$ XDPM,RE,XF,XLT,XPI,XPM,ANO,ANW

COMMON/FLUD/ FR,AMUW,AMUO,AMUP,AMO,AMOP,SWIPF,SWIPF,SWIF,

$\$ \quad$ SWIF,SWIK,SWIK,SWII,SWII,SWIINF,SWIINF,SWCT

COMMON/CONST/ NI,NO,SHK 11,SHK 12,SHKI11,SHKI12,SHK21,

$\$ \quad$ SHK22,SHK121,SHK122, AINF1,AINF2,AINFI,

$\$ \quad$ AINFI2, SWCT1,SWCT2,IFLAG

IF(ABS(FR-1.0).GT.0.000I) THEN

CALL SHOCKI(AMOP,SHK 11,SHK 12,SWIPF)

CALL SHOCK2(AMO,AMOP,SW1PF,SHK21,SHK22,SWIK)

CALL SWLMT(AMO,AMOP,SW1PF,SWCT1,SWCT2,SWCT)

IF(SWII.GT.SWCT) THEN

PRINT,$; * * * * * * * * * * * * * * * * * * * * * * * *$ WARNING 


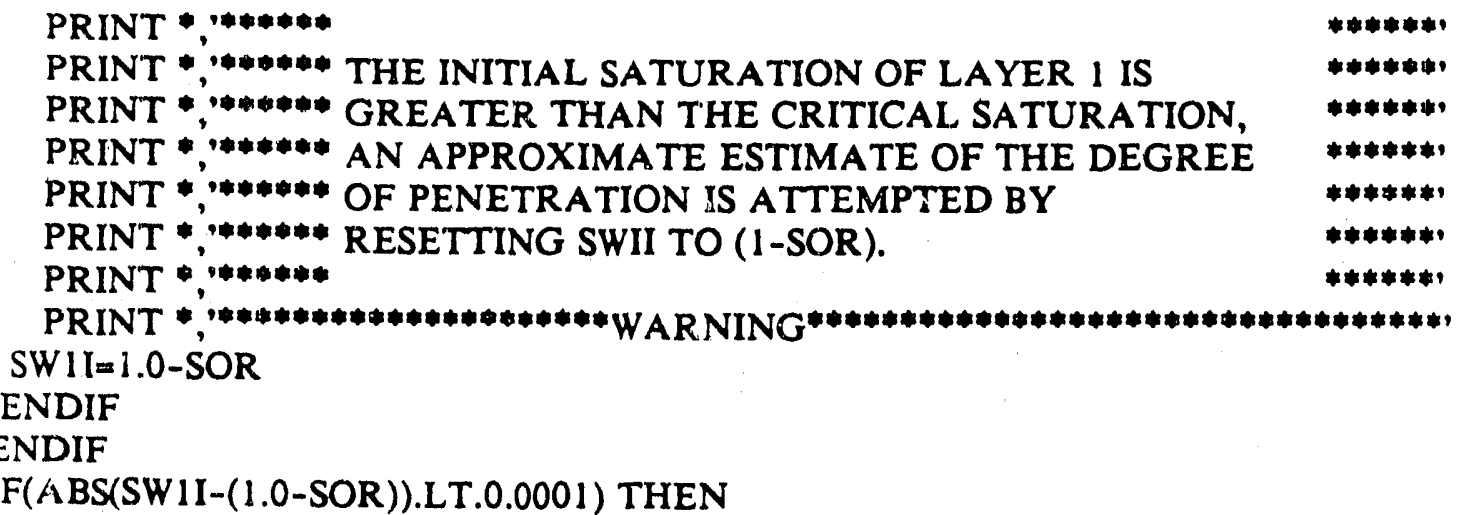

IF(ABS(SW1I-(1.0-SOR)).LT.0.0001) THEN

$R R 1=1.0$

$R I=1.0$

IF( IFLAG.EQ.1) THEN

C ---CALCULATE THE FRONTAL SATURATIONS AND THE VELOCITY RATIO OF

C OIL BANK AND GELANT FRONT WHEN THE LAYER IS WATERED-OUT

C (COREFLOOD CASES)...--

IF(ABS(FR-1.0).LT.0.0001) THEN

$X D I=1.0$

RETURN

ENDIF

$\mathrm{XDI}=1.0 / \mathrm{RI}$

ENDIF

ELSE

IF(ABS(FR-1.0).LT.0.0001) THEN

C ---CALCULATE THE FRONTAL SATURATIONS, SWAVG AND THE VELOCITY

C RATIO OF OIL BANK AND GELANT FRONT WHEN FR $=1.0 .-.-$

$\mathrm{R} I=0.0$

IF( IFLAG.EQ.1) THEN

C ---SET XDI $=1.0$ FOR COREFLOOD CASES WHEN FR=1.0..--

$\mathrm{XD} I=1.0$

ENDIF

CALL INF(AMO,AINF1,AINF2,SWIINF)

IF( SWII .LT. SWIINF) THEN

CALL SHOCK(AMO,SW1I,SHK21,SHK22,SW1F)

CALL FW(AMO,SWIF,FWIF)

CALL DFDS(AMO,SWIF,DFW1)

CALL SHOCK 1(AMOP,SHK11,SHK12,SW:PF)

CALL FW(AMO,SWII,FW1I)

CALL FW(AMOP,SWIPF,FWPI)

$R R 1=(F W 1 F-F W 1 I) * S W I P F /(F W P 1 *(S W 1 F-S W 1 I))$

$S W A V G 1=(S W 1 F+(1.0-F W 1 F) / D F W 1)-S W R$

ELSE

CALL FW(AMO,SWII,FWII)

CALL DFDS(AMO,SW1I,DFW1)

CALL FWKRW(AMO,SWII,FKRI)

CALL SHOCK I (AMOP,SHK11,SHK12,SW1PF)

CALL. FW(AMOP,SWIPF,FWPI)

RRI $=D F W 1 * S W I P F / F W P I$

IF(SWII.GT.SWIPF) THEN

RRI $=1.0$ 
SWAVGI $=S W 1 I+(1.0-F W 1 I) / D F W 1$

ELSE

SWAVGI=(SWII+(1.0-FW1I)/DFWI)-SWR

ENDIF

ENDIF

ELSE

C ---CALCULATE THE FRONTAL SATURATIONS, SWAVG AND THE VELOCITY

C RATIO OF OI', BANK AND GELANT FRONT WHEN FR>1.0...--

$R R I=1.0$

CALL SHOCK 1(AMOP,SHK 11,SHK 12,SWIPF)

CALL SHOCK2(AMO,AMOP,SW1PF,SHK21,SHK22,SW1K.)

CALL FW(AMO,SWIK,FWIK)

CALL FW(AMO,SWII,FWII)

CALL FW(AMOP,SWIPF,FWPI)

$R I=(F W I K-F W 1 I) * S W I P F /(F W P I *(S W I K-S W 1 I))$

IF( IFLAG.EQ.1) THEN

$\mathrm{XDI}=1.0 / \mathrm{R} 1$

ENDIF

CALL DFDS(AMOP,SWIPF,DFW1)

SWAVG $1=S W 1 P F+(1.0-F W P 1) / D F W 1$

ENDIF

ENDIF

RETURN

END

C

SUBROUTINE DFDS(XMO,XSW,DFW)

C THIS SUBROUTINE CALCULATES THE VALUE OF FIRST DERIVATIVE OF A

C WATER FRACTIONAL FLOW FUNCTION.

$\mathrm{C}$

COMMON/RK/ SOR,SWR,AKRWO,AKROO,PHII,PHII,AKI,AKI,XD1,

$\$ \quad X D P M, R E, X F, X L T, X P 1, X P M, A N O, A N W$

$S=(X S W-S W R) /(1.0-S W R-S O R)$

$D F W=\left(A N W * X M O * S^{* *}(A N W-1) *(1.0-S) * * A N O+A N O * X M O * S^{* *} A N W *\right.$

$\$(1.0-S) *($ ANO-1 $)) /\left(\left(\mathrm{XMO}^{*} \mathrm{~S}^{* *} \mathrm{ANW}+(1.0-\mathrm{S}) * * \mathrm{ANO}\right) * 2.0 *\right.$

$\$(1.0-S W R-S O R))$

RETUIRN

END

$\mathrm{C}$

SUBROUTINE D2FDS2(XMO,XSW,DDFW)

C THIS SUBROUTINE CALCULATES THE VALUE OF SECOND DERIVATIVE OF A C WATER FRACTIONAL FLOW FUNCTION.

$\mathrm{C}$

COMMON/RK/ SOR,SWR,AKRWO,AKROO,PHI1,PHI,AK1,AKI,XD1,

$\$ \quad X D P M, R E, X F, X L T, X P 1, X P M, A N O, A N W$

$\mathrm{S}=(\mathrm{XSW}-\mathrm{SWR}) /(1.0-\mathrm{SWR}-\mathrm{SOR})$

DDFW $=\left(\left(X M O * S^{* *} \text { ANW+(1.0-S }\right)^{* * A N O}\right) *\left(A N W *(A N W-1)^{*} X^{*} O^{*}\right.$

$\$ S^{* *}(\text { ANW }-2)^{*}(1.0-S)^{* *}$ ANO-ANO*(ANO-1)*XMO*S**ANW*

$\$ \quad(1.0-S)^{* *}($ ANO-2))-2*(ANW*XMO*S**(ANW-1)*(1.0-S)**ANO+

$\$$ ANO*XMO*S**ANW*(1.0-S)**(ANO-1) $)^{*}($ ANW*XMO*'**(ANW-1)

$\$-A N O *(1.0-S) * *(A N O-1))) /\left(\left(X M O * S^{* *} A N W+(1.0-S)^{* *}\right.\right.$ ANO) 


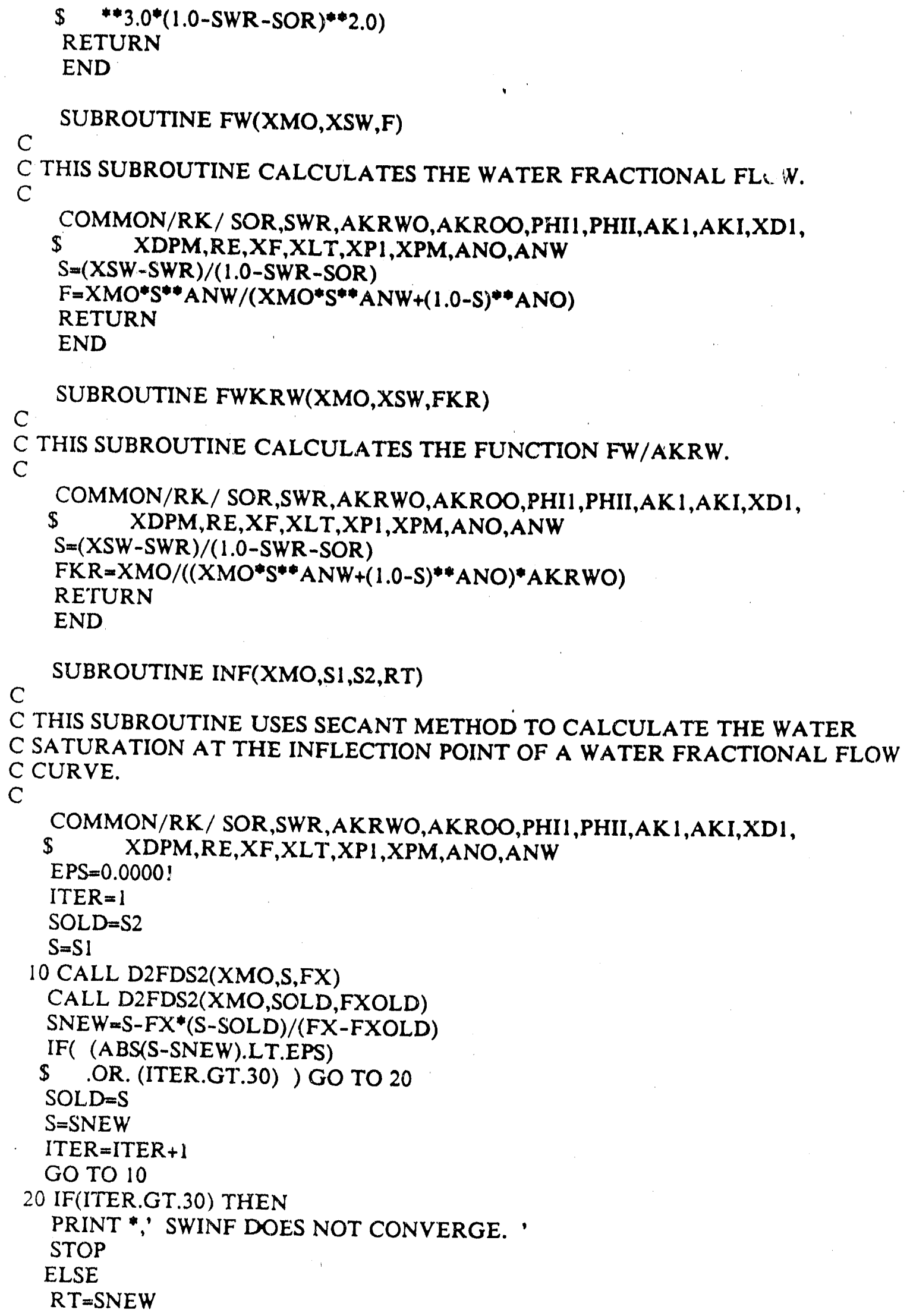


ENDIF

RETURN

END

$\mathrm{C}$

SUBROUTINE INPUT

C THIS SUBR ()UTINE READS THE NECESSARY INFORMATION FOR SUBSEQUENT

C CALCULA'IIONS.

$\mathrm{C}$

COMMON/RK/ SOR,SWR,AKRWO,AKROO,PHI1,PHII,AK1,AKI,XDI, $\$ \quad$ XDPM,RE,XF,XLT,XP1,XPM,ANO,ANW

COMMON/FLUD/ FR,AMUW,AMUO,AMUP,AMO,AMOP,SWIPF,SWIPF,SWIF, S SWIF,SWIK,SWIK,SWII,SWII,SWIINF,SWIINF,SWCT

COMMON/CONST/ NI,NO,SHK 11,SHK 12,SHKI11,SHKI12,SHK21,

$\$ \quad$ SHK22,SHKI21,SHKI22,AINF1,AINF2,AINFIl,

$\$ \quad$ AINFI2,SWCT1,SWCT2,IFLAG

PRINT *, 'INPUT SOR,SWR'

READ(*,*) SOR,SWR

PRINT *, 'INPUT SWII,SWII'

READ(*, SWII,SWII

PRINT *, 'INPUT KRWO,KROO'

READ * *) AKRWO,AKROD

PRINT ', 'INPUT ANW,ANO'

READ(*, ) ANW,ANO

PRINT *, 'INPUT PHII,PHII'

READ(*, ) PHI 1, PHII

PRINT *, 'INPUT KI,KI'

READ(*,") AKI,AKI

PRINT *, 'INPUT MUW,MUO,MUP'

READ(*;) AMUW,AMUO,AMUP

PRINT *, 'INPUT IFLAG; IFLAG=1--COREFLOOD, IFLAG=2--5-SPOT

READ(*;) IFLAG

IF(IFLAG.EQ.2) THEN

PRINT *, 'INPUT XF,XPI,XPM,RE'

READ(*;) XF,XPI,XPM,RE

ELSE

PRINT *, 'INPUT XLT'

READ $(*, *) X L T$

C -.- LET XPM=XLT FOR COREFLOOD CASES....-

$X P M=X L T$

ENDIF

PRINT *, 'INPUT SHK 11,SHK 12,SHK 111,SHK112'

READ(*,") SHK 11,SHK 12,SHKI11,SHKI12

PRINT *, 'INPUT SHK 21,SHK 22,SHK 121,SHKI22'

READY*, *) SHK 21,SHK 22,SHK121,SHKI22

PRINT *, 'INPUT AINF1,AINF2,AINFI1,AINFI2'

READ *, *) AINF1, AINF2,AINF11,AINFI2

PRINT *, 'INPUT SWCT1,SWCT2'

READ(*,") SWCT1,SWCT2

PRINT *, 'INPUT NO. OF INTERVALS'

READ $*, *)$ NI,NO

RETURN 


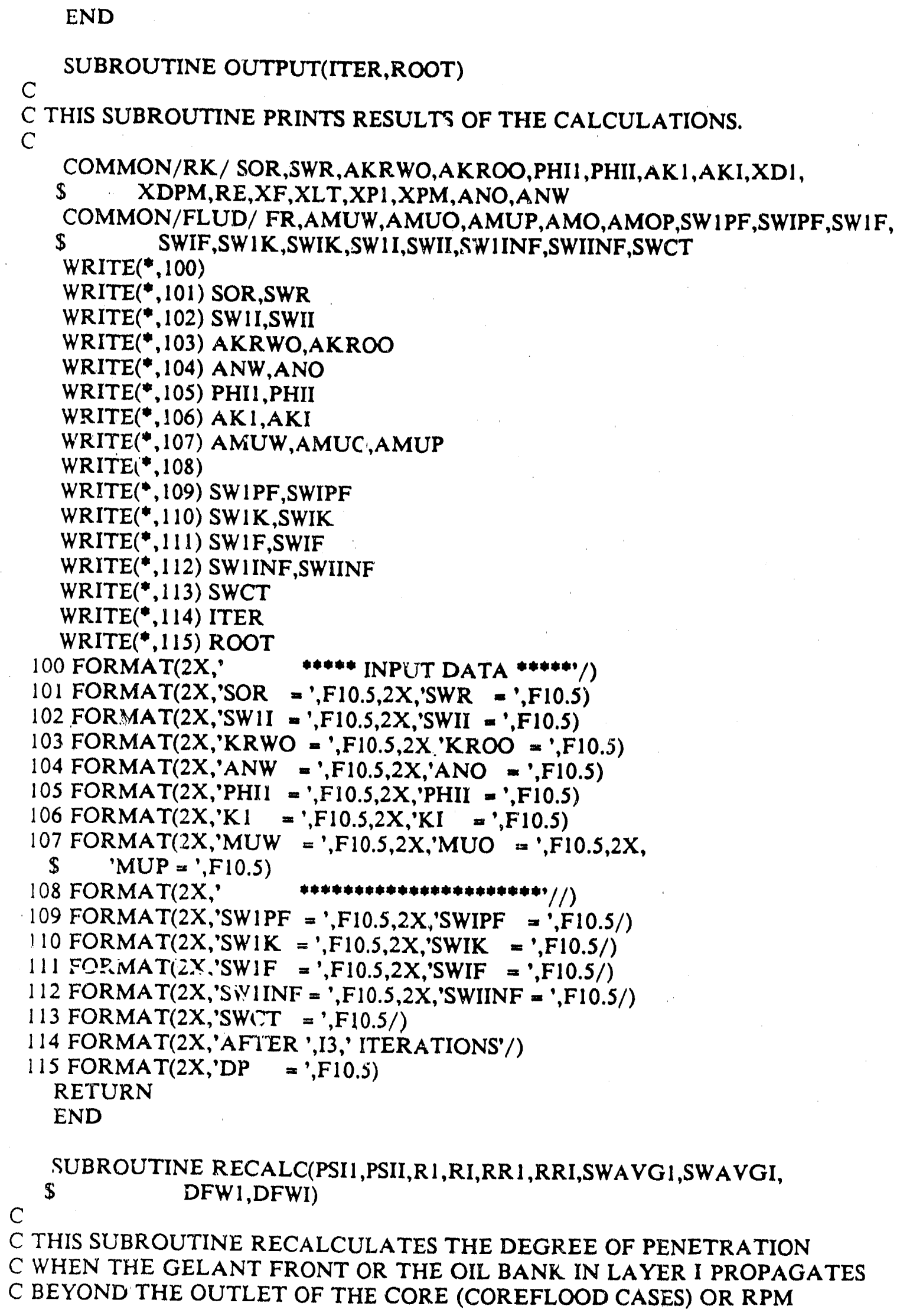


C (FIELD CASES) BY REPOSITIONING THE GELANT FRONT OR THE OIL

C BANK IN LAYER I AT THE OUTLET OF THE CORE OR RPM AND SOLVING

C FOR THE POSITION OF THE GELANT FRONT IN LAYER 1.

$\mathrm{C}$

COMMON/RK/ SOR,SWR,AKRWO,AKROO,PHI1,PHII,AKI,AKI,XD1,

\$ XDPM,RE,XF,XLT,XP1,XPM,ANO,ANW

COMMON/FLUD/ FR,AMUW,A MUO,AMUP,AMO,AMOP,SWIPF,SWIPF,SWIF,

\$ SWIF,SW1K,SWIK,SWII,SWII,SWIINF,SWIINF,SWCT

COMMON/CONST/ NI,NO,SHK 11,SHK 12,SHKI11,SHKI12,SHK21,

$\$$ SHK 22,SHKI21,SHKI22,AIINF1,AINF2,AINFI1,

$\$ \quad$ AINFI2,SWCT1,SWCT2,IFLAG

C ----REPOSITION THE GELANT FRONT OR THE OIL BANK IN LAYER I----

IF(IFLAG.EQ.1) THEN

IF(ABS(FR-1.0).LT.0.0001) THEN

$\mathrm{XDI}=1.0$

ELSE

$\mathrm{XDI}=1.0 / \mathrm{RI}$

ENDIF

ELSE

IF(ABS(FR-1.0).LT.0.0001) THEN

$X D I=X D P M$

ELSE

$\mathrm{XDI}=\mathrm{XDPM} / \mathrm{RI}$

ENDIF

ENDIF

C ---CALCULATE THE LEFT-HAND-SIDE OF THE THEORETICAL

C EQUATION (LAYER I)----

CALL ALHSF(XDI,RI,PSII,SWAVGI,DFWI,ALHS)

C .... USE SECANT METHOD TO SOLVE FOR THE DEGREE OF PENETRATION

C OF GELANTS INTO LAYER 1-..-

EPS=1.0E-07

ITER $=1$

$X=0.0001$

$\mathrm{XOLD}=0.5$

C ---EVALUATE THE RIGHT-HAND-SIDE OF THE THEORETICAL

C EQUATION (LAYER 1)--.-

$10 \mathrm{XD} 1=\mathrm{X}$

CALL RHSF(R1,PSI1,SWA VG1,DFW1,RHS)

$F X=A L$ HS-RHS

$\mathrm{XD} I=\mathrm{XOLD}$

CALL RHSF(R1,PSI1,SWAVG1,DFW1,RHSO)

FXOLD $=$ ALHS-RHSO

$\mathrm{XNEW}=\mathrm{X}-\mathrm{FX} *(\mathrm{X}-\mathrm{XOLD}) /(\mathrm{FX}-\mathrm{FXOLD})$

IF( (ABS(X-XNEW) .LT. EPS)

$\$$.OR. (ITER.GT.30) ) GO TO 20

$X O L D=X$

$X \approx X N E W$

ITER $=$ ITER+1

GO TO 10

20 IF(ITER.GT.30) THEN

PRINT *;*************WARNING************************1

PRINT *' DP DOES NOT CONVERGE AFTER 30 ITERATIONS.' 


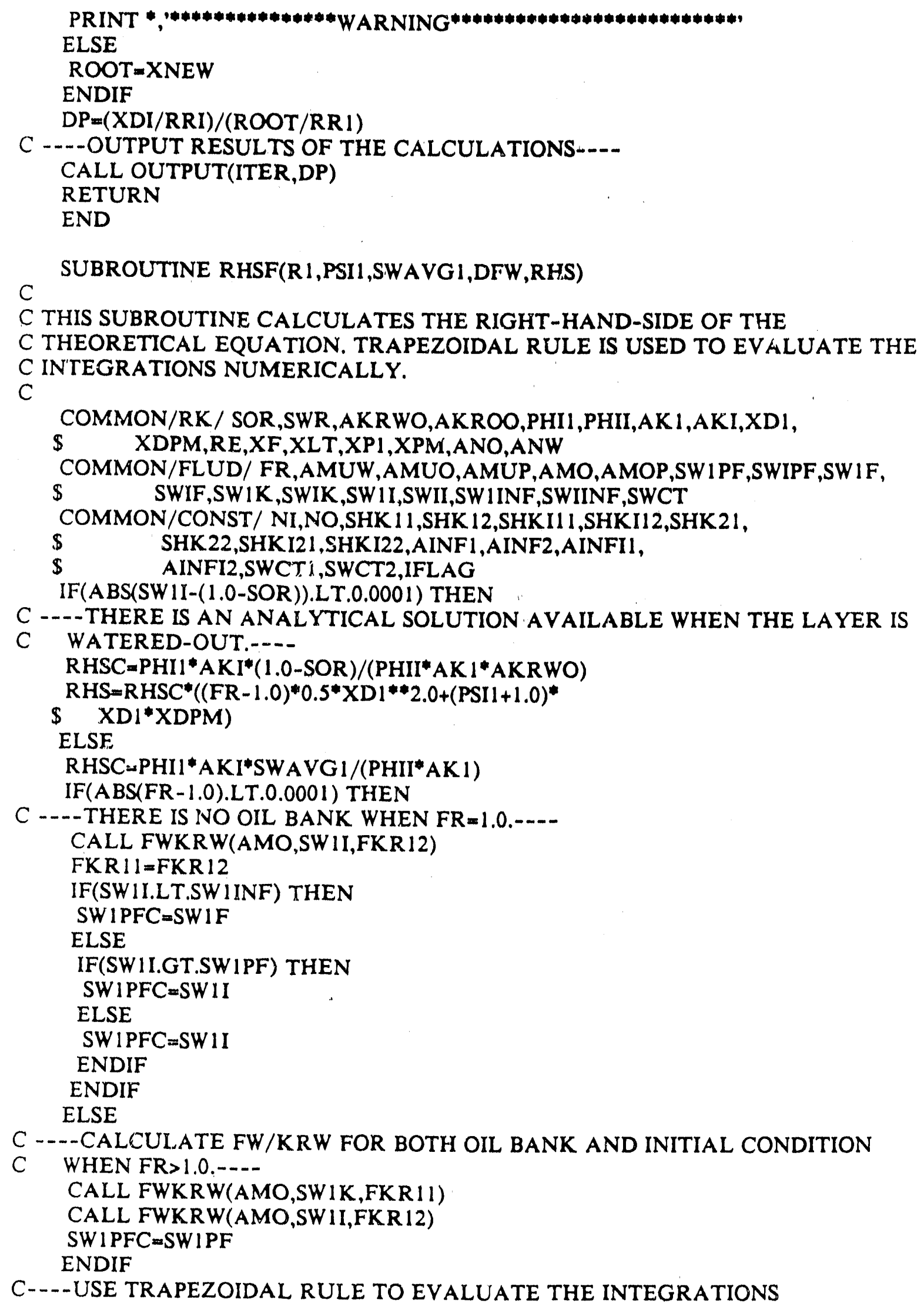


C NUMERICALLY.-.--

$H=X D 1 / N O$

SUM $=0.0$

DO $100 \quad I=1, \mathrm{NO}-1$

$T=I^{*} H$

TD=T/DFW

CALL TRAPEZ(0.0,T,SW1PFC,NI,AMOP,TD,ARI)

IF(RI.LT.0.0001) THEN

$\mathrm{R} \perp \mathrm{T}=0.0$

ELSE

$R I T=R I * T$

ENDIF

RHS $=(A R 1 * F R+F K R \perp 1 *(R I T-T)+F K R 12 *(X D P M$

$\left.\left.\$ \quad-R I T+P S I 1^{*} X D P M\right)\right) *$ RHSC

$S U M=S U M+R H S$

100 CONTINUE

TD-XDI/DFW

CALL TRAPEZ(0.0,XD1,SW1PFC,NI,AMOP,TD,AR1)

IF(RI.LT.0.0001) THEN

$R \mid R D I=0.0$

ELSE

$R|R D|=R 1 * X D 1$

ENDIF

$\mathrm{RHSB}=(\mathrm{AR} 1 * \mathrm{FR}+\mathrm{FKR} 11 *(\mathrm{R} 1 \mathrm{RD} 1-\mathrm{XD} 1)+\mathrm{FKR} 12 *(\mathrm{XDPM}$

$\left.\left.\$ \quad-R \mid R D 1+P S I I^{*} X D P M\right)\right)^{*} R H S C$

RHSA $=($ FKR $12 *$ (XDPM

$\$+$ PSI $1 * X D P M)) *$ RHSC

$\mathrm{RHS}=(\mathrm{H} / 2.0)^{*}\left(\mathrm{RHSB}+2.0^{*} \mathrm{SUM}+\mathrm{RHSA}\right)$

ENDIF

RETURN

END

SUBROUTINE SECANT(XMO,TD,X,S1,S2,RT)

$\mathrm{C}$

C THIS SUBROUTINE USES SECANT METHOD TO SOLVE FOR THE WATER

C SATURATION PROFILE.

C

COMMON/RK/ SOR,SWR,AKRWO,AKROO,PHI!,PHI,AK1,AKI,XD1,

$\$ \quad X D P M, R E, X F, X L T, X P 1, X P M, A N O, A N W$

$E P S=0.0001$

ITER $=1$

SOLD $=\mathrm{S} 2$

$\mathrm{S}=\mathrm{S} 1$

10 CALL XDS(S,XMO,X,TD,FX)

CALL XDS(SOLD,XMO,X,TD,FXOLD)

$\mathrm{SNEW}=\mathrm{S}-\mathrm{FX}$ *(S-SOLD)/(FX-FXOLD)

IF( (ABS(S-SNEW).LT.EPS)

$\$$.OR. (ITER.GT.100) ) GO TO 20

SOLD $=S$

$S=$ SNEW

ITER $=$ ITER +1

GO TO 10 


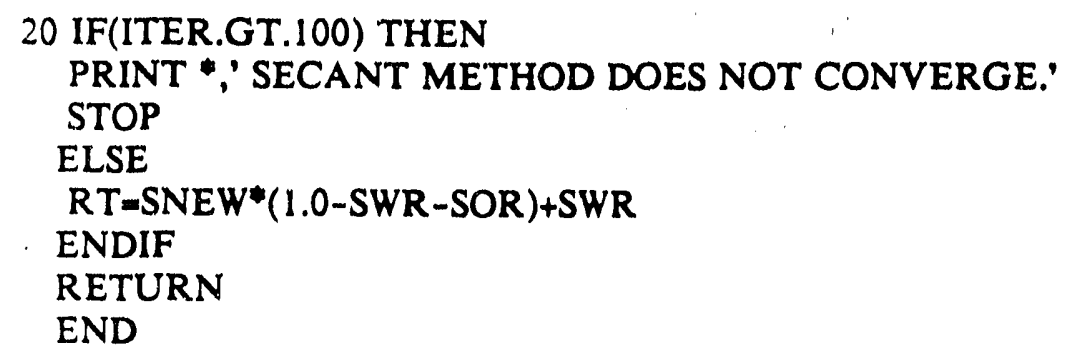

C THIS SUBROUTINE USES SECANT METHOD TO CALCULATE THE FRONTAL C SATURATIONS OF GELANTS.

$\mathrm{C}$

COMMON/RK/ SOR,SWR,AKRWO,AKROO,PHI1,PHII,AK1,AKI,XDI, $\$ \quad$ XDPM,RE,XF,XLT,XP1,XPM,ANO,ANW

EPS $=0.000001$

ITER $=1$ 


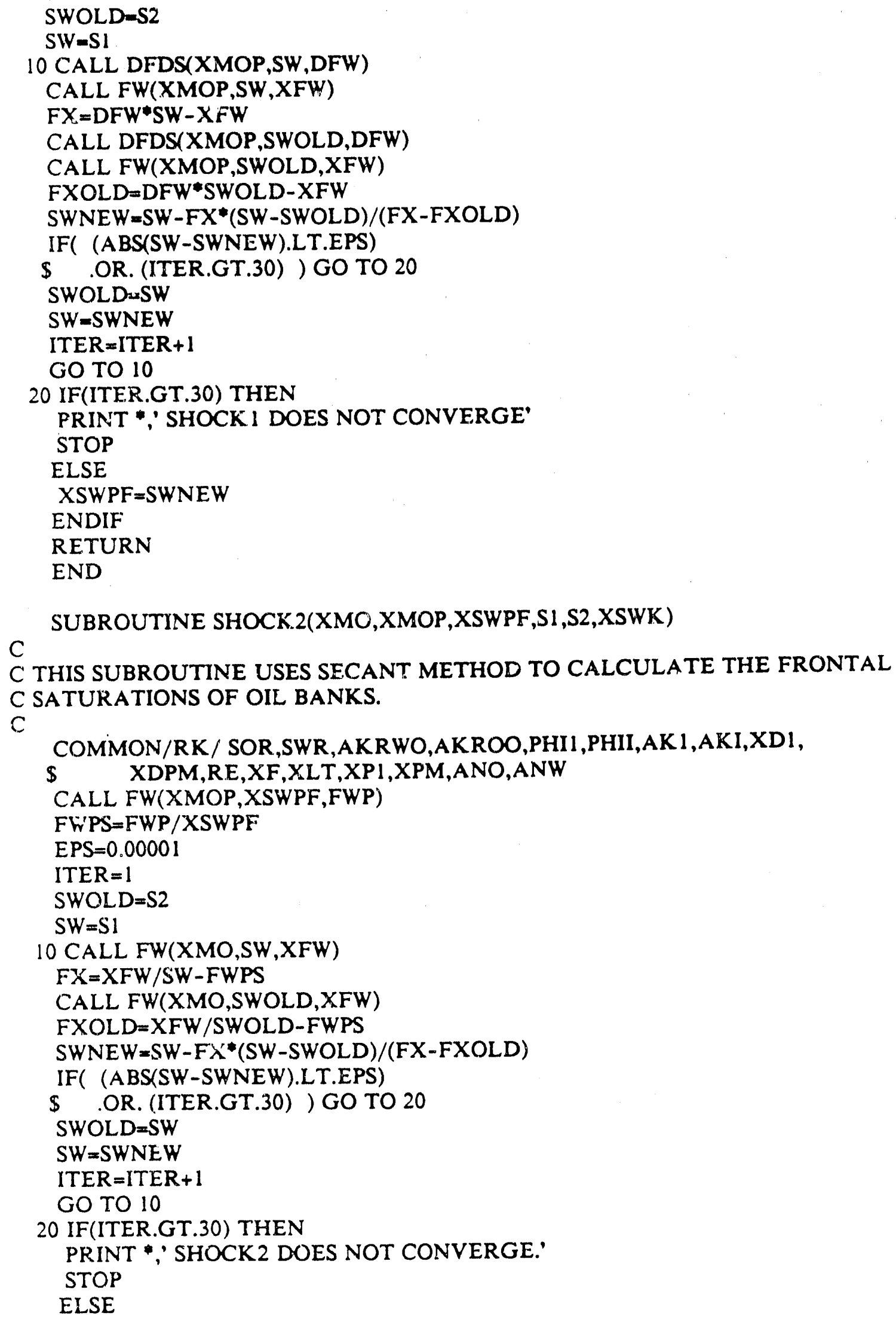




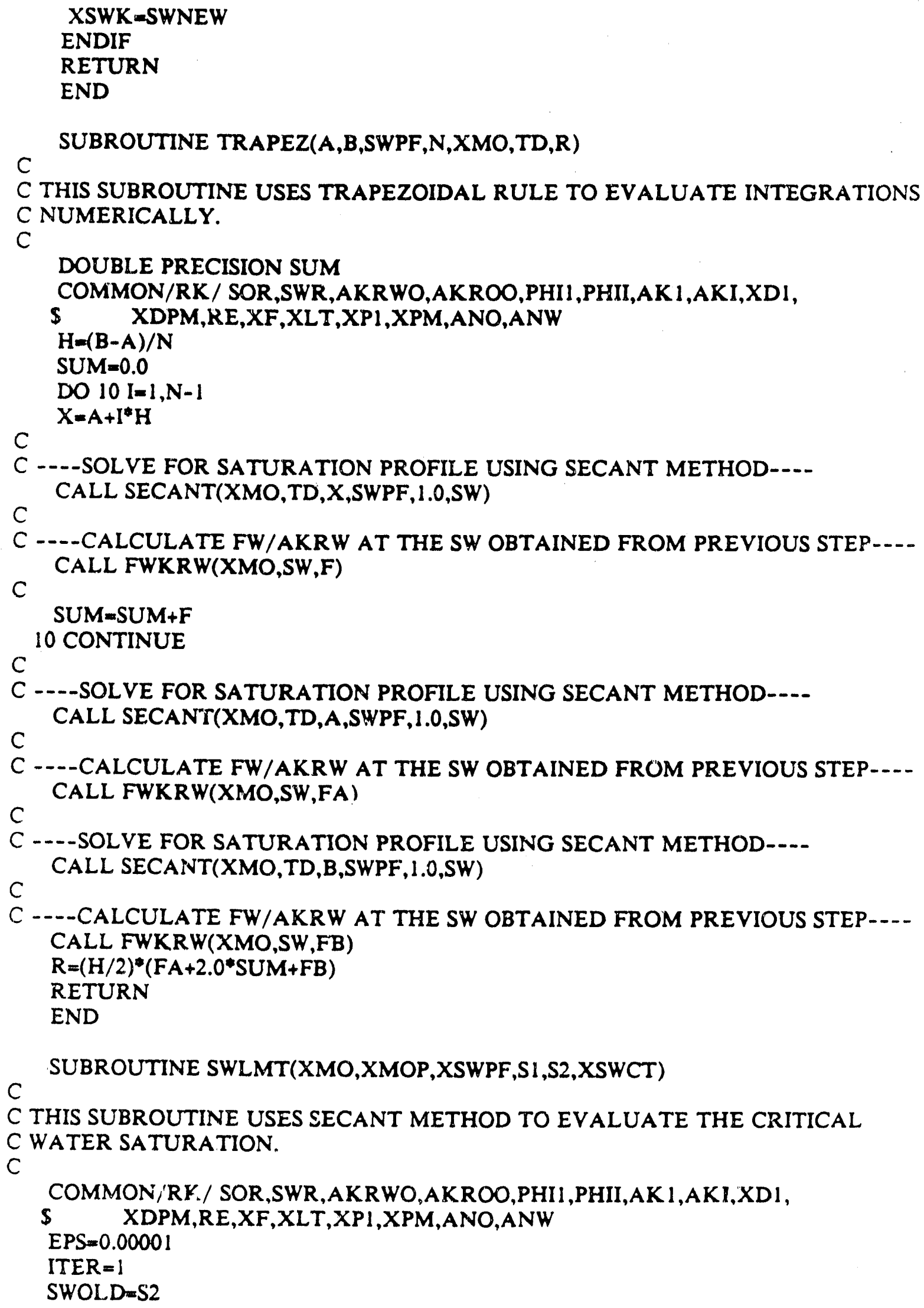




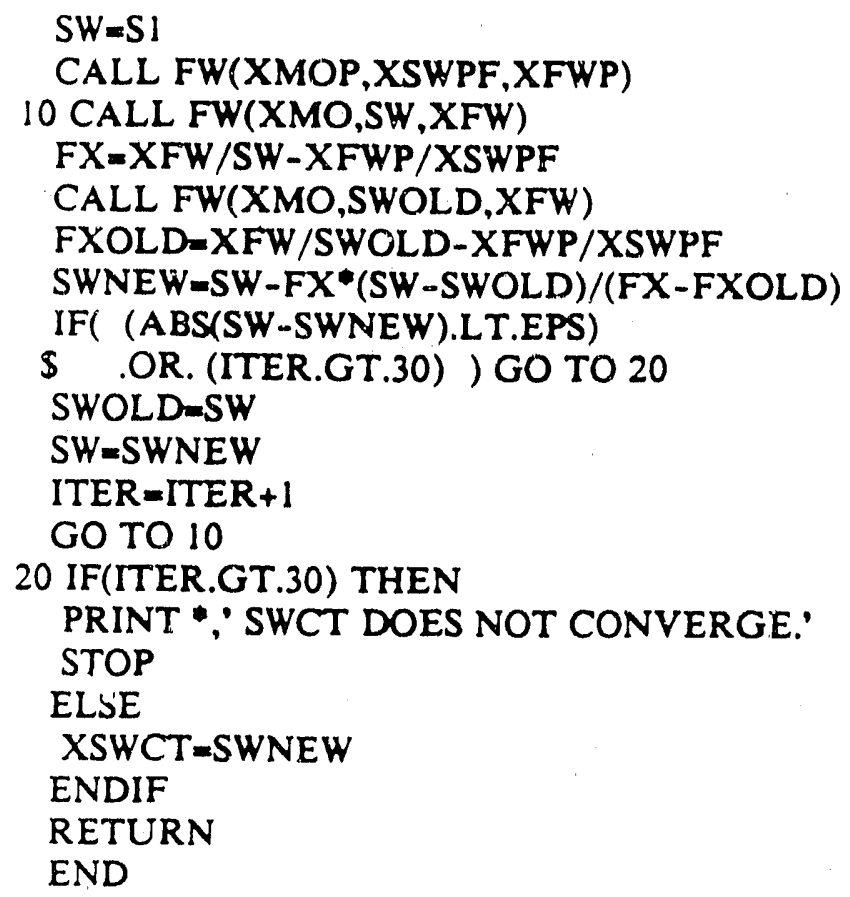


APPENDIX $\mathrm{H}$

FORMULATIONS FOR NUMERICAL MODEL 
In this appendix, the two-phase flow equation used in the simulator is briefly described. The finite difference formulations of the equation, both in linear and radial cases, are derived.

\section{Two-Phase Flow Equation}

In the one-dimension linear case after combining the continuity and the Darcy equations, the flow equations for both phases are

$$
\begin{aligned}
& \frac{\partial}{\partial x}\left[\rho_{w} \lambda_{w}\left(\frac{\partial P_{w}}{\partial x}\right)\right]+q_{w}=\frac{\partial\left(\rho_{u} S_{w} \phi\right)}{\partial t} \\
& \frac{\partial}{\partial x}\left[\rho_{n} \lambda_{n}\left(\frac{\partial P_{n}}{\partial x}\right)\right]+q_{n}=\frac{\partial\left(\rho_{n} S_{n} \phi\right)}{\partial t}
\end{aligned}
$$

(assuming the gravity forces acting on the system are negligible). The variables, $x$ and $t$, are space and time coordinates, respectively. The variable, $\rho$, is the density of the fluid, $P$ is the pressure, $S$ is the saturation and $\phi$ is the porosity of the rock, and $q$ is the mass flow rate per unit volume. The subscripts, $w$ and $n$, denote the wetting and nonwetting phases, respectively. The mobility, $\lambda$, is defined as

$$
\lambda\left(S_{w}\right)=\frac{k \cdot k_{r}}{\mu}
$$

which is the ratio or ff fective permeability to the viscosity of fluid.

Adding Eqs. $\mathrm{H}-1$ and $\mathrm{H}-2$ and making some rearrangements, one obtains

$$
\begin{aligned}
& \frac{1}{\rho_{w}} \frac{\partial}{\partial:} \cdot\left[\rho_{w} \lambda_{w}\left(\frac{\partial P_{w}}{\partial x}\right)\right]+\frac{1}{\rho_{n}} \frac{\partial}{\partial x}\left[\rho_{n} \lambda_{n}\left(\frac{\partial P_{n}}{\partial x}\right)\right]=-Q_{\imath}+\frac{1}{\rho_{w}} \frac{\partial}{\partial x}\left[\rho_{w} \lambda_{w}\left(\frac{\partial P_{c}}{\partial x}\right)\right] \\
& \quad+\frac{\partial \phi}{\partial t}-\phi S_{w} C_{w} \frac{\partial P_{c}}{\partial t}+\left(\phi S_{w} C_{w}+\phi S_{n} C_{n}\right) \frac{\partial P_{n}}{\partial t}
\end{aligned}
$$

where $Q_{1}$ is total volumetric flow rate, $C$ is compressibility of the fluid, and $P_{c}$ is capillary pressure:

$$
\begin{aligned}
Q_{t} & =\frac{q_{n}}{\rho_{n}}+\frac{q_{w}}{\rho_{w}} \\
C & =\frac{d \rho}{d P} \cdot \frac{1}{\rho} \\
P_{c} & =P_{w}-P_{n}
\end{aligned}
$$


becomes

By assuming 1) $\mathrm{P}_{c}$ is constant; 2) $\phi$ is constant; and 3) fluids are incompressible, Eq. $\mathrm{H}-4$

$$
\frac{1}{\rho_{u^{\prime}}} \frac{\partial}{\partial x}\left[\rho_{w} \lambda_{u^{\prime}}\left(\frac{\partial P_{n}}{\partial x}\right)\right]+\frac{1}{\rho_{n}} \frac{\partial}{\partial x}\left[\rho_{n} \lambda_{n}\left(\frac{\partial P_{n}}{\partial x}\right)\right]=-Q_{t}
$$

or in general form:

$$
\frac{1}{\rho_{w}} \nabla \cdot\left[\rho_{w} \lambda_{w} \nabla P_{n}\right]+\frac{1}{\rho_{n}} \nabla \cdot\left[\rho_{n} \lambda_{n} \nabla P_{n}\right]=-Q_{t}
$$

which is the basic equation for the finite difference formulations.

\section{Numerical Approximation}

In both linear and radial cases, layers are discretized to $\mathrm{m}$ finite sections. For a regular node, $\mathrm{i}$, as shown in Fig. H-1, Eq. H-9 can be approximated as:

\section{a. Linear Case:}

$$
\begin{aligned}
& \left\{\frac{1}{\rho_{w}} \frac{\partial}{\partial x}\left[\rho_{w} \lambda_{w}\left(\frac{\partial P_{n}}{\partial x}\right)\right]\right\}_{i} \\
& \quad=\left(\frac{1}{\rho_{w}}\right)_{i}\left[\left(\rho_{w} \lambda_{w}\right)_{i+1 / 2} \frac{P_{n, i+1}-P_{n, i}}{(\Delta x)^{2}}-\left(\rho_{w} \cdot \lambda_{w}\right)_{i-1 / 2} \frac{P_{n, i}-P_{n, i-1}}{(\Delta x)^{2}} !\right. \\
& \left\{\frac{1}{\rho_{n}} \frac{\partial}{\partial x}\left[\rho_{n} \lambda_{n}\left(\frac{\partial P_{n}}{\partial x}\right)\right]\right\}_{i} \\
& \quad=\left(\frac{1}{\rho_{n}}\right)_{i}\left[\left(\rho_{n} \lambda_{n}\right)_{i+1 / 2} \frac{P_{n, i+1}-P_{n, 2}}{(\Delta x)^{2}}-\left(\rho_{n} \lambda_{n}\right)_{i-1 / 2} \frac{P_{n, 2}-P_{n, i-1}}{(\Delta x)^{2}} !\right.
\end{aligned}
$$

\section{b. Radial Case:}

$$
\begin{aligned}
\left\{\frac{1}{\rho_{w}}\left(\frac{1}{r}\right) \frac{\partial}{\partial r}\left[\rho_{w} \lambda_{w}\left(r \frac{\partial P_{w}}{\partial r}\right)\right]\right\}_{i} & =\left\{\frac{4}{\rho_{w}} \frac{\partial}{\partial r^{*}}\left[r^{*} \rho_{w} \lambda_{w}\left(\frac{\partial P_{w}}{\partial r^{*}}\right)\right]\right\}_{i} \\
=\left(\frac{4}{\rho_{w}}\right)\left(\frac{1}{r_{i+1 / 2}^{*}-r_{i-1 / 2}^{*}}\right) & {\left[\left(\rho_{u} \lambda_{w}\right)_{i+1 / 2} r_{i+1 / 2}^{*} \frac{P_{n, i+1}-P_{n, i}}{r_{i+1}^{*}-r_{i}^{*}}\right.} \\
& \left.-\left(\rho_{w} \lambda_{w}\right)_{z-1 / 2} r_{i-1 / 2}^{*} \frac{P_{n, i}-P_{n, 1-1}}{r_{i}^{*}-r_{i-1}^{*}}\right]
\end{aligned}
$$



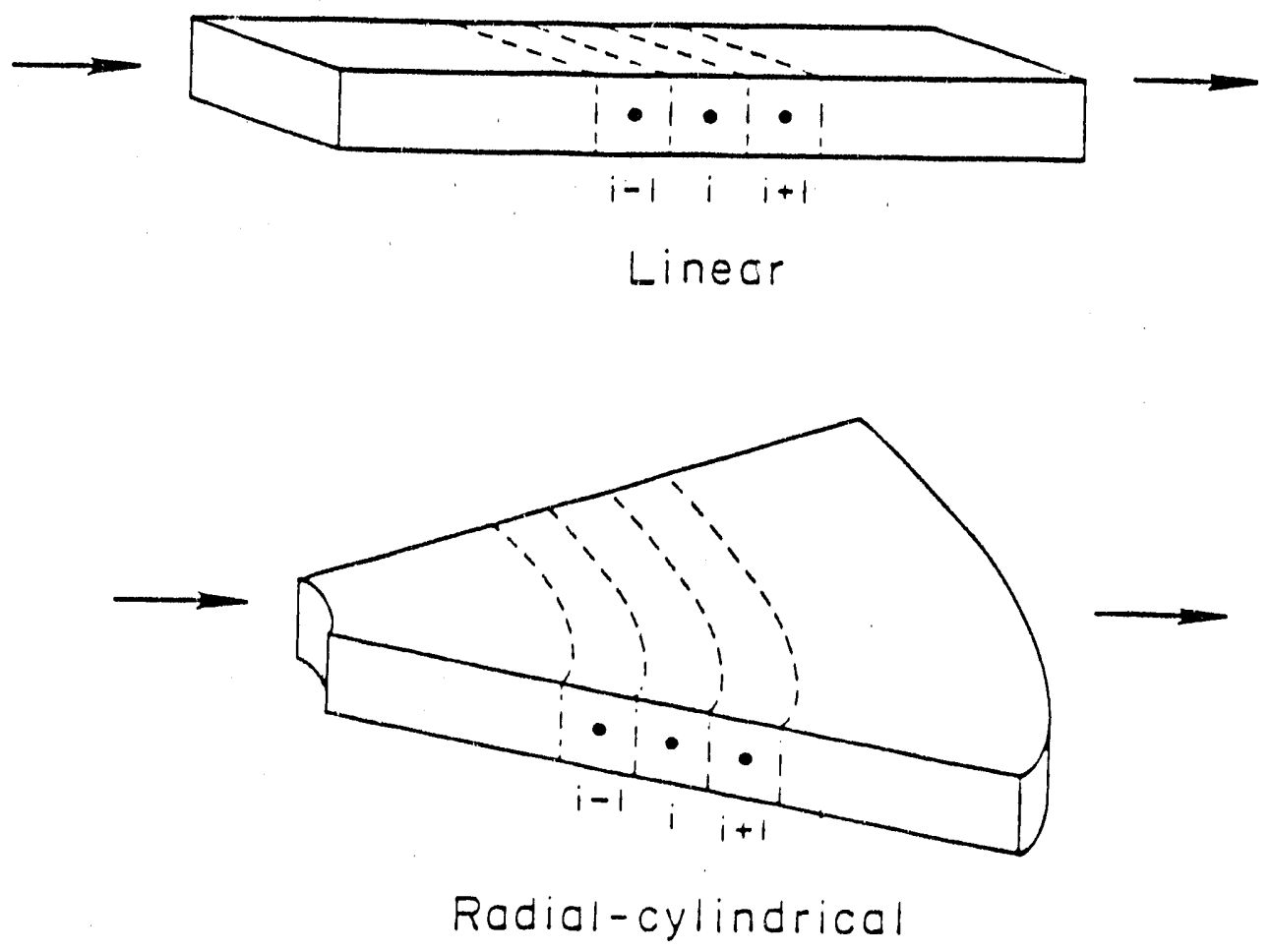

Fig. H-1. Grid-Point Distribution in Space. 


$$
\begin{aligned}
&\left\{\frac{1}{\rho_{n}}\left(\frac{1}{r}\right) \frac{\partial}{\partial r}\left[\rho_{n} \lambda_{n}\left(r \frac{\partial P_{n}}{\partial r}\right)\right]\right\}_{i}=\left\{\frac{4}{\rho_{n}} \frac{\partial}{\partial r^{*}}\left[r^{*} \rho_{n} \lambda_{n}\left(\frac{\partial P_{n}}{\partial r^{*}}\right)\right]\right\}_{i} \\
&=\left(\frac{4}{\rho_{n}}\right)\left(\frac{1}{r_{i+1 / 2}^{*}-r_{i-1 / 2}^{*}}\right)\left[\left(\rho_{n} \lambda_{n}\right)_{i+1 / 2} r_{i+1 / 2}^{*} \frac{P_{n, i+1}-P_{n, i}}{r_{i+1}^{*}-r_{i}^{*}}\right. \\
&\left.-\left(\rho_{n} \lambda_{n}\right)_{i-1 / 2} r_{i-1 / 2}^{*} \frac{P_{n, 2}-P_{n, i-1}}{r_{i}^{*}-r_{i-1}^{*}}\right]
\end{aligned}
$$

where

$$
\begin{aligned}
& r_{i+1 / 2}^{*}=\frac{r_{i+1}^{2}-r_{i}^{2}}{\ln \left(r_{i+1}^{2} / r_{i}^{2}\right)} ; \quad r_{i-1 / 2}^{*}=\frac{r_{i}^{2}-r_{i-1}^{2}}{\ln \left(r_{i}^{2} / r_{i-1}^{2}\right)} \\
& r_{i}^{*}=r_{i}^{2}
\end{aligned}
$$

To avoid instabilities, the upstream weighting method is used. The midpoint mobilities, $\lambda_{i+1 / 2}$ and $\lambda_{i-1 / 2}$, are replaced by $\lambda_{i+1}$ and $\lambda_{i}$, the mobilities at their upstream nodes. Since an iterative solving method is used, the two-point upstream weighting on the mobilities is not necessary. The reason for using $\mathrm{r}^{2}$ instead of $\mathrm{r}$ in the radial case is to ensure the material balance. For example, the volume of a block cannot be discretized directly from the partial differential form:

$$
(2 \pi r d r \cdot h)_{i}=2 \pi r_{i}\left(r_{i+1 / 2}-r_{i-1 / 2}\right) \cdot h \neq \pi\left(r_{i+1 / 2}^{2}-r_{i-1 / 2}^{2}\right) \cdot h=\pi \cdot d r^{2}
$$

Throughout this study, the constant pressure boundary was applied. One can simply replace the boundary node (points 1 and $n$ ) equations by $P_{1}=P_{a}$ and $P_{n}=P_{b}$ where the values of $P_{a}$ and $P_{b}$ are given.

The final matrix form is

$$
a_{i j}^{k} P_{n, 2}^{k}=b_{i}
$$

where

$$
\begin{aligned}
a_{i j, i \neq j}^{k} & =\text { Factor }_{i-1 / 2} \cdot \frac{\lambda_{w, j}^{k}+\lambda_{n, j}^{k}}{(\Delta x)^{2}} \text { if } j=i-1 \\
a_{i j, i \neq j}^{k} & =\text { Factor }_{i+1 / 2} \cdot \frac{\lambda_{w, j-1}^{k}+\lambda_{n, j-1}^{k}}{(\Delta x)^{2}} \text { if } j=i+1 \\
a_{i i}^{k} & =-a_{i, i+1}^{k}-a_{i, i-1}^{k}
\end{aligned}
$$


where

$$
\begin{aligned}
& \text { For Radial Case: } \text { Factor }_{i+1 / 2}=2 \pi \frac{r_{i+1}-r_{i}}{\ln \left(r_{i+1} / r_{i}\right)} \cdot \Delta r \\
& \text { For Linear Case: } \text { Factor }_{i+1 / 2}=1.0
\end{aligned}
$$

At boundary nodes, $a_{i j}=1, a_{i j, j} \neq j=0$, and $b_{i}=P_{i}$. The $k$ is an iterative index. Since $\lambda^{k}$ is a function of saturation, $S_{w}$, the mobility, $\lambda$, should have an average value between two given time levels, $\lambda=$ $f\left(S_{w i}\right)$.

\section{IMPES Procedure}

As shown in Fig. H-2, from the known saturations at time level $m$, the final matrix can be constructed from which a set of intermediate values of $P_{n, i}^{k}$ is abtained. The saturations at tinie level $\mathrm{m}+1$ can be calculated by the discretized forms of Eqs. $\mathrm{H}-1$ and $\mathrm{H}-2$. If the saturation change in any node is greater than 0.1 , the time step is reduced and the $P_{n}$ calculation is repeated until the values of $P_{n, i}^{k}$ are converged. To reduce the calculation time, the program has an automatic adjustment for selecting an optimal time step. is

It should be noted, in the radial case, that the equivalent and discretized equation of Eq. $\mathrm{H}-1$

$$
\begin{aligned}
4 \pi r_{i+1 / 2}^{*} \lambda_{w, i} \frac{P_{w, i+1}-P_{w, i}}{r_{i+1}-r_{i}} & -4 \pi r_{i-1 / 2}^{*} \lambda_{w, i-1} \frac{P_{w, i}-P_{w, i-1}}{r_{i}-r_{i-1}} \\
= & \pi\left(r_{i+1 / 2}^{*}-r_{i-1 / 2}^{*}\right)\left[\phi \frac{S_{w}^{m+1}-S_{w}^{m}}{\Delta t}-\frac{q_{w}}{\rho_{w}}\right]
\end{aligned}
$$

where the saturation at time level $m+1, S_{w}^{m+1}$, can be calculated explicitly if the pressure profile is solved. 


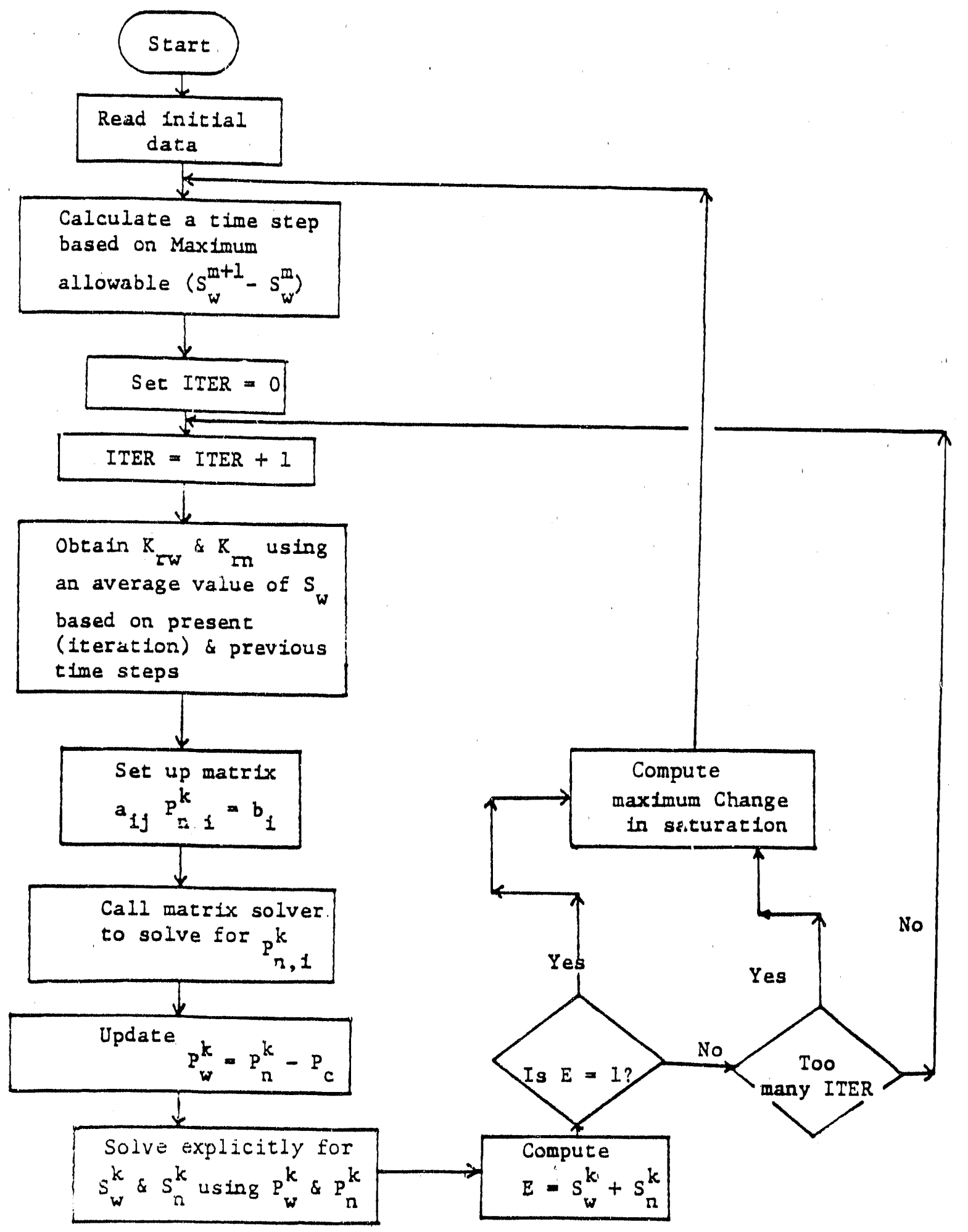

Fig. H-2. Flow Chart of IMPES Method. 

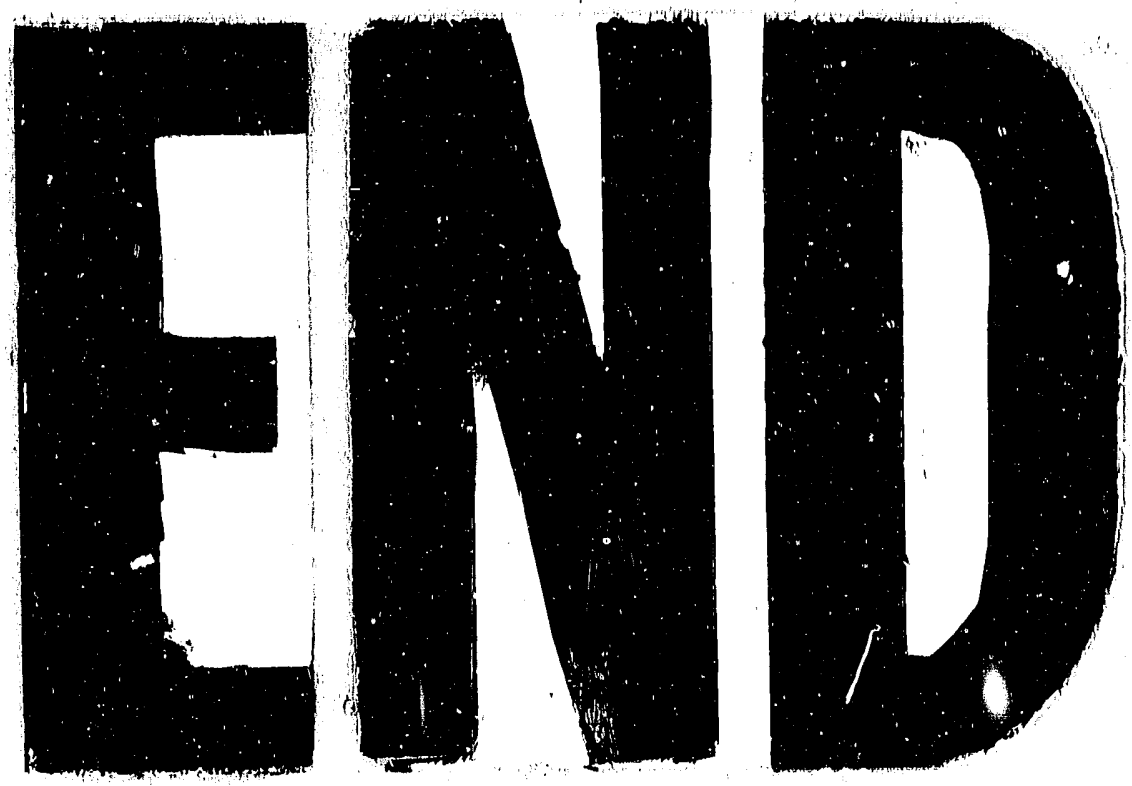

4

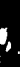

$$
\text { . }
$$
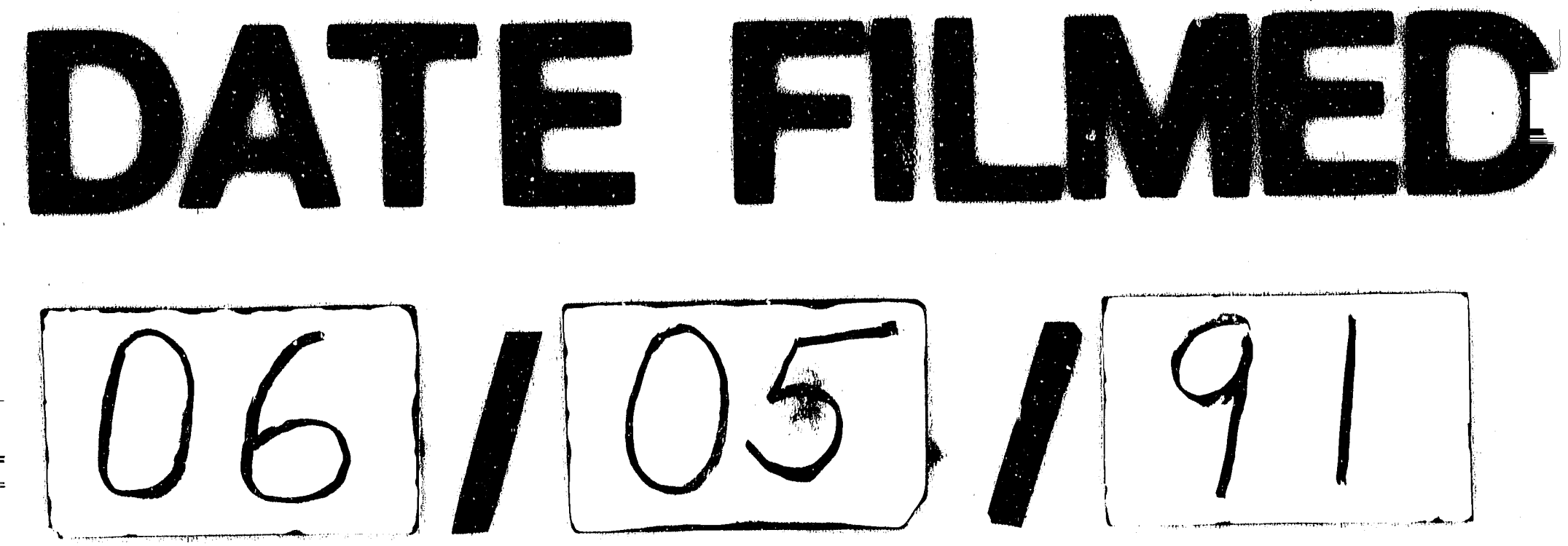
